\title{
DECORRELATION TIME ANALYSIS USING DYNAMIC LIGHT SCATTERING WITH OPTICAL COHERENCE TOMOGRAPHY IN AN IN VIVO MOUSE TUMOUR MODEL
}

By

\author{
Timothy Wan Hei Luk, B.A.Sc. in Engineering Science \\ University of Toronto \\ Toronto, Ontario, Canada, 2009
}

\author{
A thesis presented to Ryerson University \\ in partial fulfillment of the \\ requirements for the degree of \\ Master of Science \\ in the Program of \\ Biomedical Physics
}

Toronto, Ontario, Canada, 2013

(C) Timothy Wan Hei Luk, 2013 


\section{Author's Declaration}

I hereby declare that I am the sole author of this thesis. This is a true copy of the thesis, including any required final revisions, as accepted by my examiners.

I authorize Ryerson University to lend this thesis to other institutions or individuals for the purpose of scholarly research.

I further authorize Ryerson University to reproduce this thesis by photocopying or by other means, in total or in part, at the request of other institutions or individuals for the purpose of scholarly research.

I understand that my thesis may be made electronically available to the public. 


\begin{abstract}
Decorrelation Time Analysis Using Dynamic Light Scattering with Optical Coherence

Tomography in an in vivo Mouse Tumour Model
\end{abstract}

Timothy Wan Hei Luk, Master of Science, 2013

Department of Physics, Program of Biomedical Physics

Ryerson University

Optical coherence tomography (OCT) is an imaging modality that uses near infrared light interferometry for non-invasive, near-histological resolution imaging at the micron level. Concepts from dynamic light scattering (DLS) can be adapted to OCT to detect and measure the motions in the target tissue. Tissue dynamics can be observed by measuring the speckle decorrelation time (DT) of the tissue. DT analysis was performed in a preclinical study to demonstrate the repeatability and feasibility of using DLS-OCT to observe mouse tumours undergoing cisplatin treatment over a 48-hour period. Differences in the average DT data were observed for control and cisplatin-injected mice. Image segmentation based on DT values was also performed to subtract the DT contributions of pixels at blood vessel locations, resulting in the improvement of average DT calculations of the tumour tissue. The results presented are a preliminary step to analyzing and monitoring tumour growth and treatment response in vivo. 


\section{Acknowledgements}

I would like to thank my supervisors Dr. Victor X.D. Yang and Dr. Michael C. Kolios for their patience and support throughout this research project. Even though we encountered some obstacles with the original research direction, both of you continued to provide encouragements for me to persevere and do my very best. I believe the research presented in this thesis was done well due to the shortened time frame, and I could not have accomplished this without my supervisors' help. I truly appreciate the opportunities I had as a graduate student working with these exceptional mentors.

I would also like to acknowledge others who have been extremely helpful in my research, such as Dr. Adrian Mariampillai and his help with the decorrelation time analysis and MATLAB expertise, Golnaz Farhat and her direction with her in vitro and in vivo work with decorrelation time that paved the way for my project, and Barry (Dr. Bear) Vuong and his advice and assistance on all OCT-related research. Of note, I would also like to mention certain individuals that helped with my mice experiments: Dr. Brian C. Wilson (for allowing me to use his lab space and mice for my experiments), Emily Chen (for performing the window chamber surgeries), Elizabeth Berndl (for growing the tumour cells), Azusa Maeda (for helping me with the tail vein injections), Ahmed El Kaffas (for helping with the H\&E imaging), Bahar Davoudi and Leigh Conroy (for their general lab assistance at PMH), Arthur Worthington (for all the research-related orders), members of the Kolios Group, and friends in the Biomedical Physics program for their insights. 
I also appreciate the gracious support, encouragements, and insightful discussions from the other members of the Biophotonics and Bioengineering Lab at Ryerson. These include Dr. Beau Standish, Dr. Carry Sun, Michael Leung, Kenneth (Code Monkey) Lee, Mark Harduar (who always comes through in the clutch), Joe Yu (thank you for your jokes and always distracting me), Antonio ("kids these days") Mauro, Benjamin Lee, Marjan Razani, Kate Lee, Shaima Abid, Jenny Yeow (thank you for all the administrative help, and the research and non-research related discussions), and many others.

Lastly, I would like to express gratitude to the friends and family who have been extremely supportive of my research. I know I may have missed out on certain events due to my research commitments, but I appreciate your understanding. Many thanks to my parents and brother for always being there and encouraging me to work my hardest to achieve what I've been called to do. 


\section{Dedication}

This research work is dedicated to all my friends and family who have supported me throughout these years. 


\section{Table of Contents}

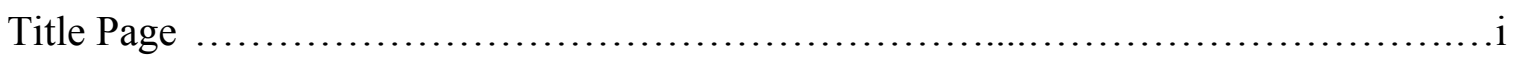

Author's Declaration .....................................................................

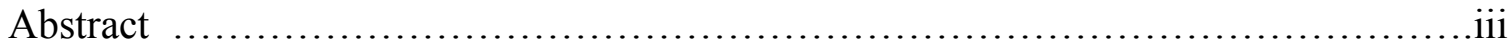

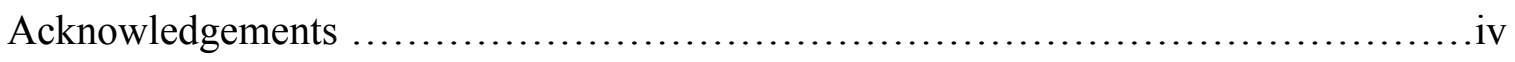

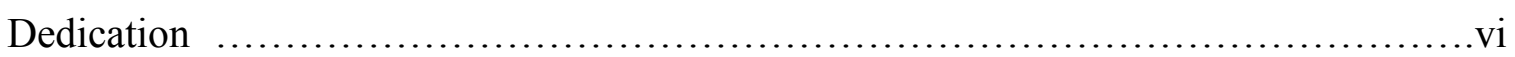

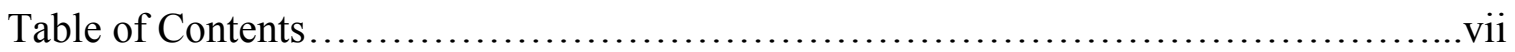

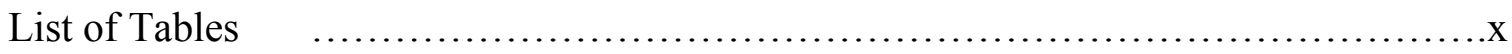

List of Figures ....................................................................

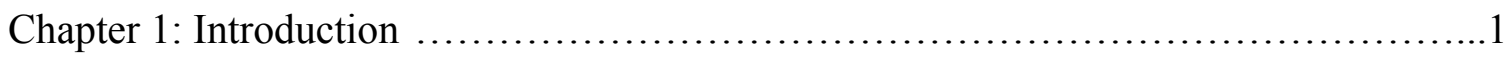

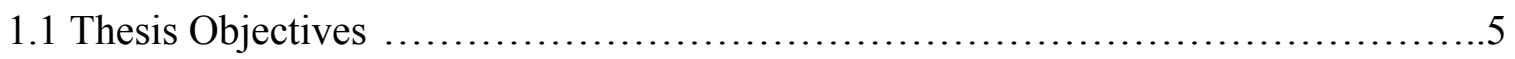

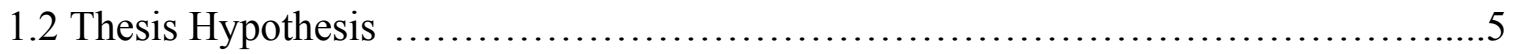

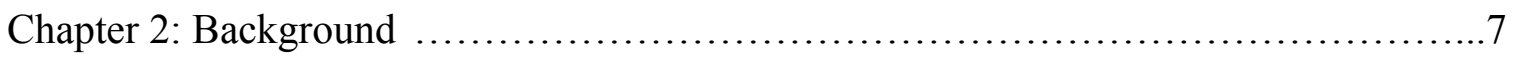

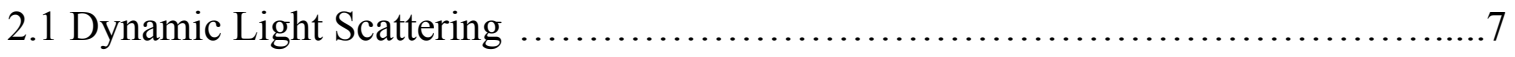

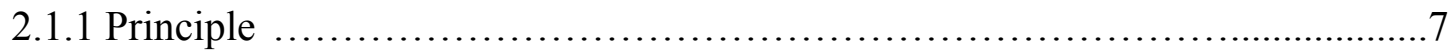

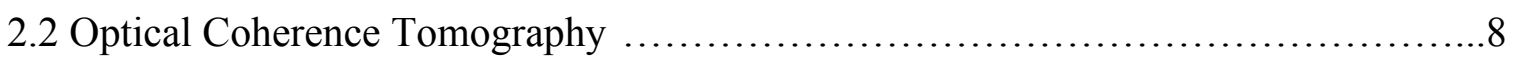

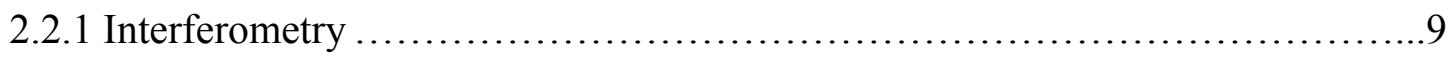

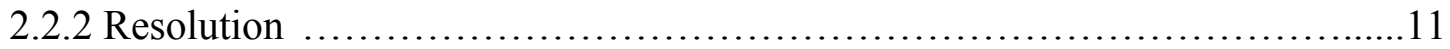

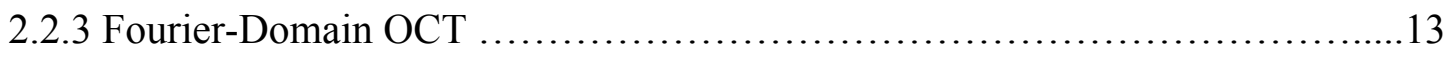

2.2.3.1 Spectral-Domain OCT …....................................... 15

2.2.3.2 Swept-Source OCT .............................................15

2.2.4 OCT Research Applications ...............................................16

2.2.4.1 OCT in Ophthalmology ........................................... 16

2.2.4.2 Intravascular OCT …......................................... 17

2.2.4.3 OCT in Oncology ................................................... 19

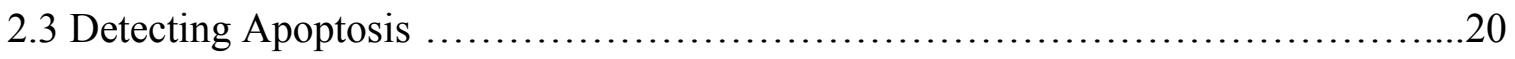

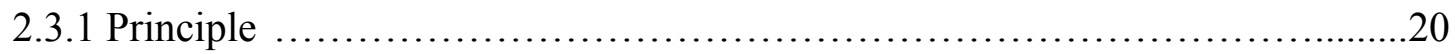

2.3.2 Apoptosis Detection Using OCT …....................................21 


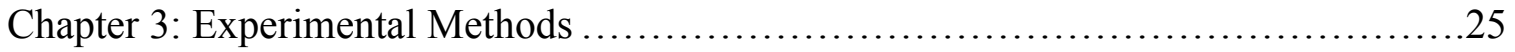

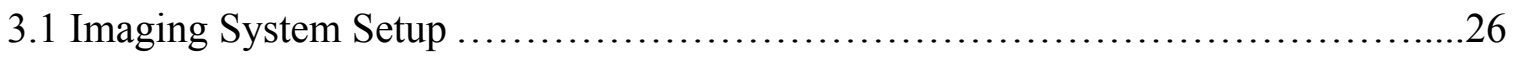

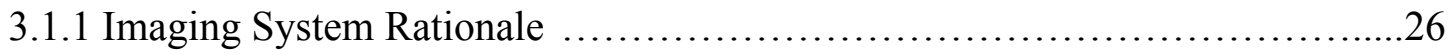

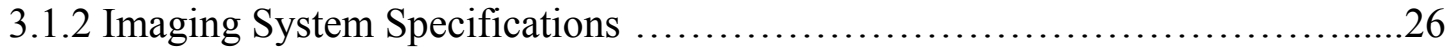

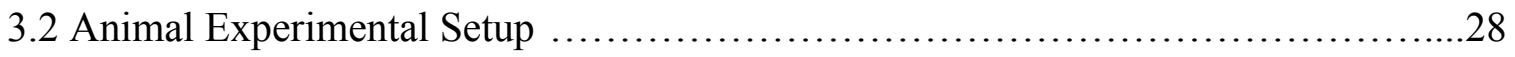

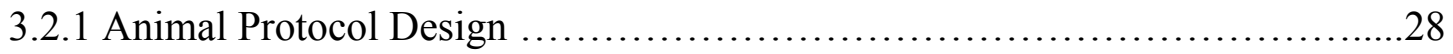

3.3 Data Acquisition and Analysis ....................................................

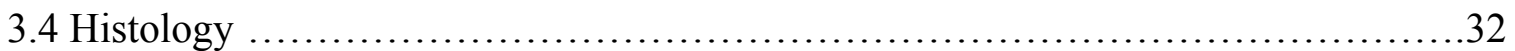

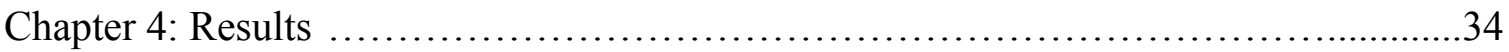

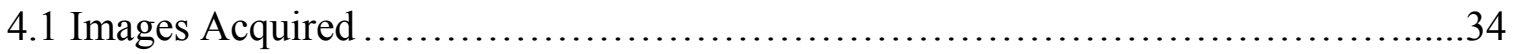

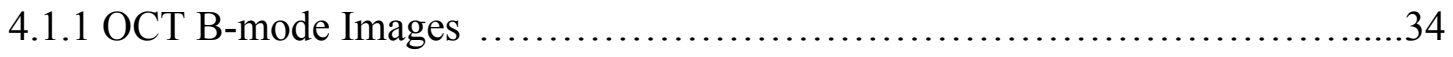

4.1.2 Decorrelation Time Maps …................................................

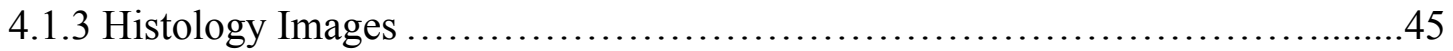

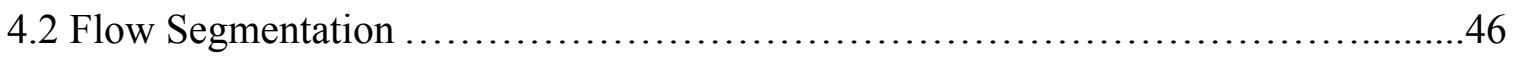

4.2.1 Quantitative Analysis of Thresholding ....................................... 49

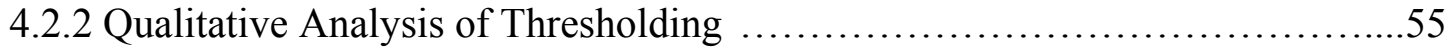

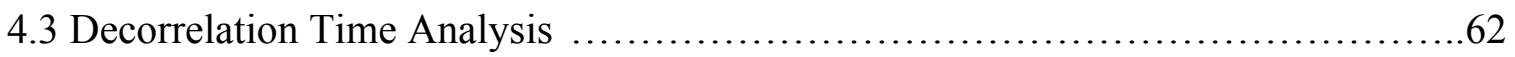

4.3.1 Decorrelation Time Averages Across Imaging Planes ..........................64

4.3.2 Decorrelation Time Averages for Control and Treated Mice ....................71

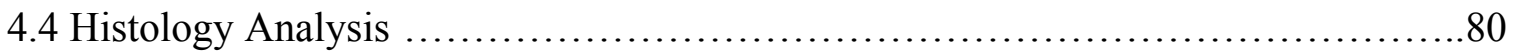

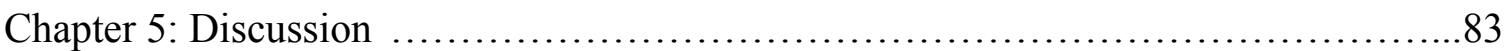

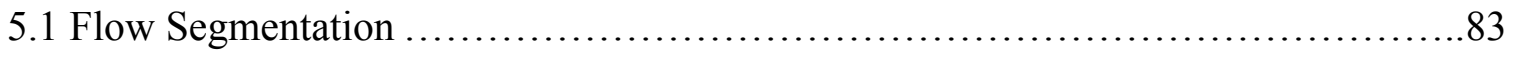

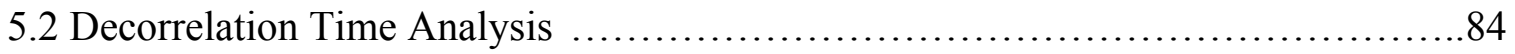

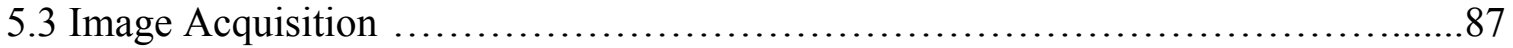

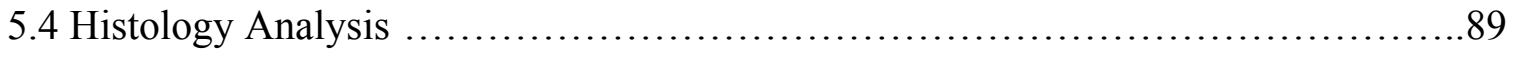

Chapter 6: Conclusions and Future Work ........................................ 91

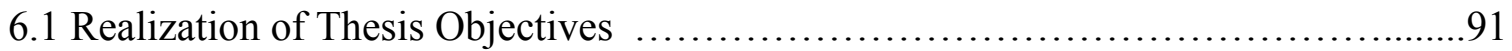


Appendix A: Excerpt of the Animal Protocol for the Mice Experiment ................96

Appendix B: Images of the Mice Experiment .................................... 98

Appendix C: Sample MATLAB Code for Formulating DT Maps ......................99

Appendix D: Heterodyne Approach ....................................... 102

Appendix E: Threshold Charts .......................................... 106

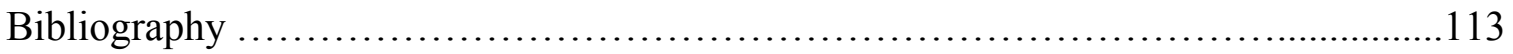




\section{List of Tables}

Table 3-1: Mice experiment chart detailing the mice and the performed treatments and sacrifice date

Table 3-2: Revised mice experiment chart detailing the mice and the performed

treatments and sacrifice date

Table 4-1: Decorrelation times taken at a long DT pixel and short DT pixel for Mouse 2

and Mouse 6 over ten 100-frame sections

Table 4-2: An excerpt of the chart data corresponding to Figure 4-7 for Mouse 6 from

Day 0 to Day 2

Table 4-3: An excerpt of the chart data corresponding to Figure 4-8 for Mouse 10 from

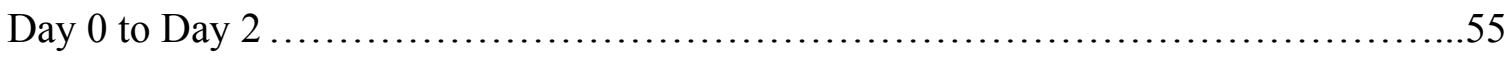

Table 4-4: The four categories indicating the ROI selection and thresholding ...........71

Table 4-5: An excerpt of the Student's t-test to compare the means of the average DT

values recorded for the control and cisplatin mice in Figure $4-17 \ldots \ldots \ldots \ldots \ldots \ldots \ldots . \ldots . \ldots . \ldots . \ldots$

Table E-1: A full summary chart of the data corresponding to Table 4-2 for Mouse 6 from

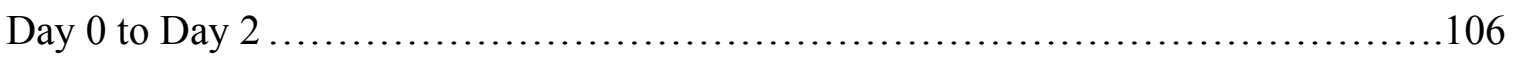

Table E-2: A full summary chart of the data corresponding to Table 4-3 for Mouse 10

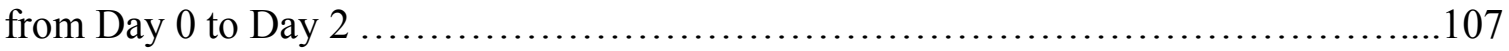

Table E-3: Actual average DT values and pixel counts for all four categories

corresponding to Table 4-4

Table E-4: Student's t-test for comparing the means for control and cisplatin mice for all

four categories corresponding to Table 4-5 


\section{List of Figures}

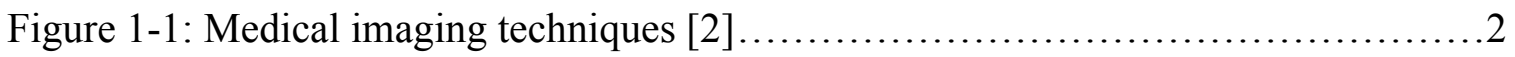

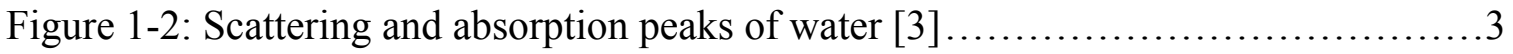

Figure 2-1: Michelson interferometer and the depiction of long and short coherence

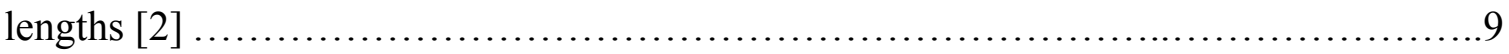

Figure 2-2: Time-domain OCT and an OCT image of a fingernail $[24,25] \ldots \ldots \ldots \ldots . .11$

Figure 2-3: The difference between using a low NA and high NA lens [2] ..............12

Figure 2-4: TD-OCT images of coronary atherosclerotic plaque types $[27] \ldots \ldots \ldots \ldots . .18$

Figure 2-5: Results of $40 \mathrm{MHz}$ ultrasound imaging of centrifuged apoptotic cells [43] ...20

Figure 2-6: Hematoxylin and eosin (H\&E) stained sections obtained from cisplatin

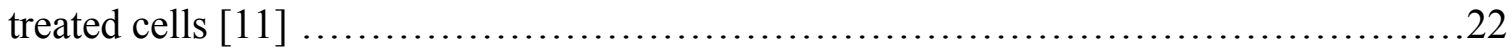

Figure 2-7: Average intermediate scattering functions and decorrelation time $[11] \ldots \ldots .23$

Figure 2-8: Comparing decorrelation in skin versus tumour tissue $[12] \ldots \ldots \ldots \ldots \ldots \ldots . .24$

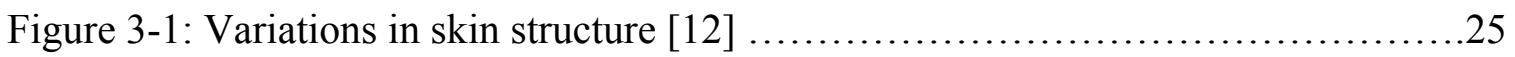

Figure 3-2: Schematic for the high-speed wavelength-swept semiconductor laser with a

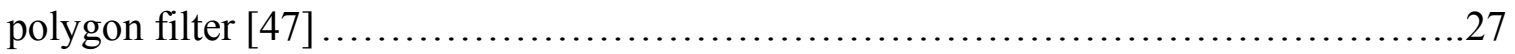

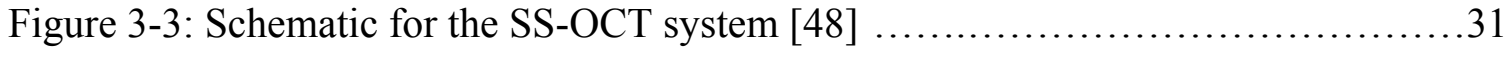

Figure 4-1: A cross-sectional (b-mode) image of the tumour for Mouse 6 and $10 \ldots \ldots . .35$

Figure 4-2: DT map of the mouse tumour produced from OCT b-mode images using a

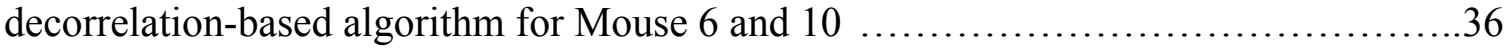

Figure 4-3: Graphs analyzing power spectrum and decorrelation time for Mouse 6 .....38

Figure 4-4: Graphs analyzing power spectrum and decorrelation time for Mouse $10 \ldots . .41$

Figure 4-5: An example hematoxylin and eosin stained histology slide of the tumour for 
Mouse 6 and 10

Figure 4-6: DT map (without threshold applied) of Mouse 6 and $10 \ldots \ldots \ldots \ldots \ldots \ldots . . . . .48$

Figure 4-7: Average DT values of different thresholds for Mouse 6 .................50

Figure 4-8: Average DT values of different thresholds for Mouse 10 .................53

Figure 4-9: DT maps of Mouse 6 with different thresholds ..........................56

Figure 4-10: DT maps of Mouse 10 with different thresholds .........................59

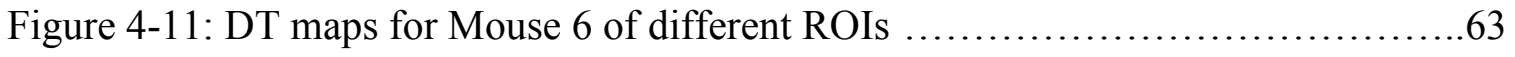

Figure 4-12: DT maps for Mouse 10 of different ROIs ...........................65

Figure 4-13: Average DT values of 3 ROIs of Mouse 6 across different planes .........67

Figure 4-14: Average DT values of 3 ROIs of Mouse 10 across different planes .......69

Figure 4-15: DT maps for the four categories for Mouse 6 ....................... 72

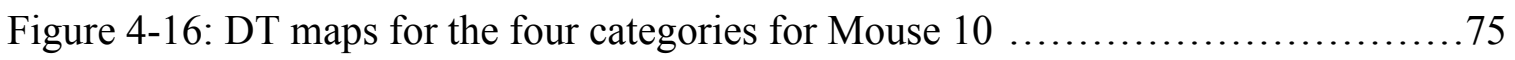

Figure 4-17: Average DT plots for five mice over the 48-hour treatment period .........77

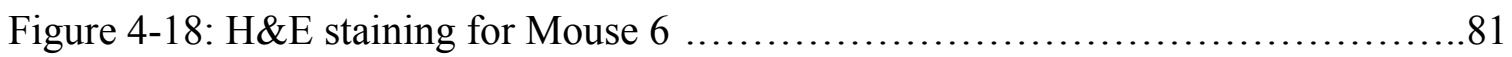

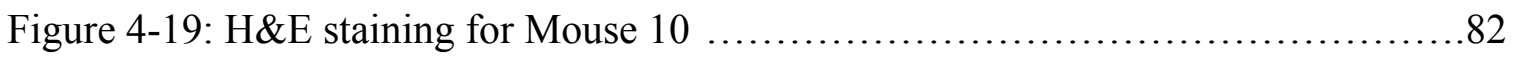

Figure 5-1: Mirroring artifact observed in this cross-sectional b-mode image ...........88

Figure B-1: SS-OCT bench-top setup at Princess Margaret Hospital ..................98

Figure B-2: Dorsal skin window chamber mouse tumour imaging ...................98

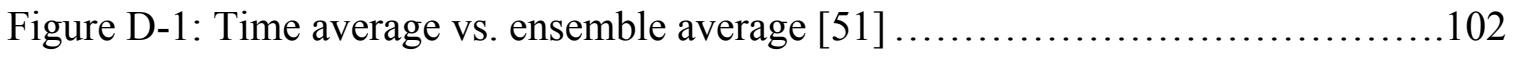




\section{Chapter 1: Introduction}

Medical imaging is a field of study that encompasses many processes and techniques that are used for disease diagnosis and analysis for clinical and research purposes. In the clinic, medical imaging is vital for the management of patient diseases. In the research field, medical imaging is essential in the study of disease development and monitoring the disease to aid the clinician's work. Minimally invasive imaging techniques have been developed and standardized as three-dimensional imaging modalities in diagnostic medicine. Such imaging techniques include magnetic resonance imaging (MRI), ultrasound (US), X-ray computed tomography (CT), positron emission tomography (PET), single-photon emission computed tomography (SPECT), and other radiography, nuclear medicine, and tomography techniques. A common limitation to these techniques is the low spatial resolution, which typically is in the millimetre range. Another imaging modality (confocal microscopy, CM) can provide micron image resolution, but lacks the penetration depth (less than 1 millimetre) of these medical imaging techniques (millimetres to centimetres) [1]. 


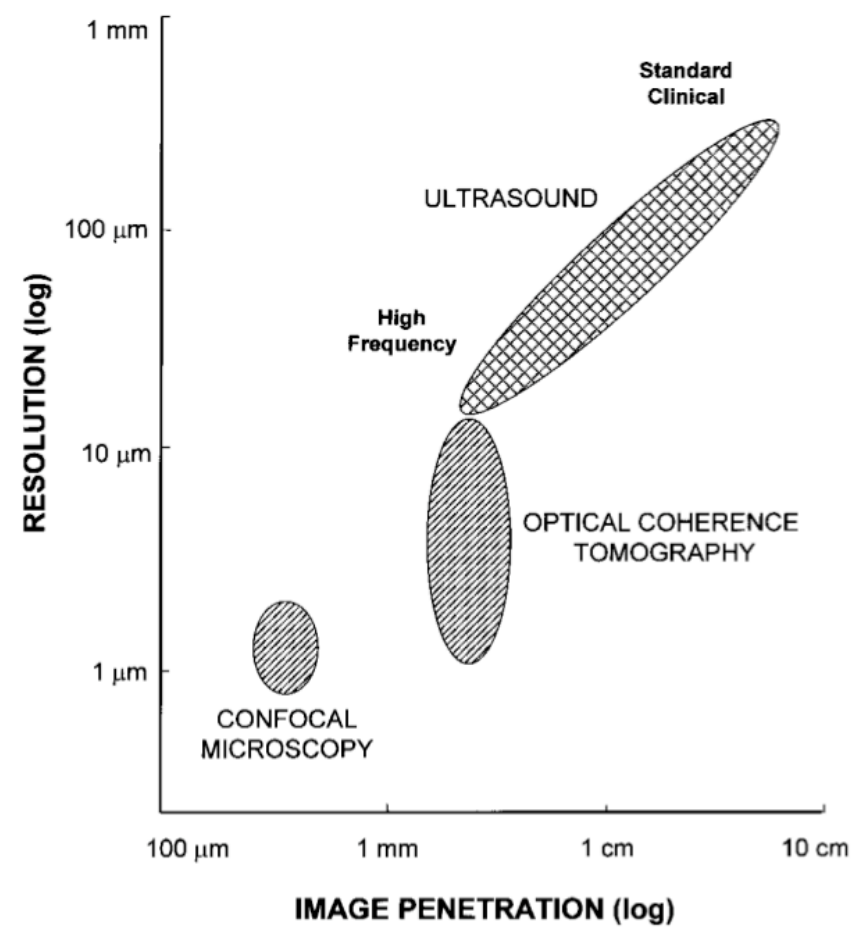

Figure 1-1: Medical imaging techniques (US, CM, and OCT) and their resolution and penetration depths [2].

Optical coherence tomography (OCT) is an imaging technology developed in the 1990s [2]. Using near infrared light interferometry, OCT offers near-histological resolution (ranging from submicron to a few microns) and is a non-invasive (or minimally invasive, depending on the application) imaging modality. Moreover, it provides images that cannot be obtained by other means due to its high resolution imaging while maintaining a depth of field. Figure 1-1 compares the resolution and penetration depths of confocal microscopy, ultrasound, and optical coherence tomography. 


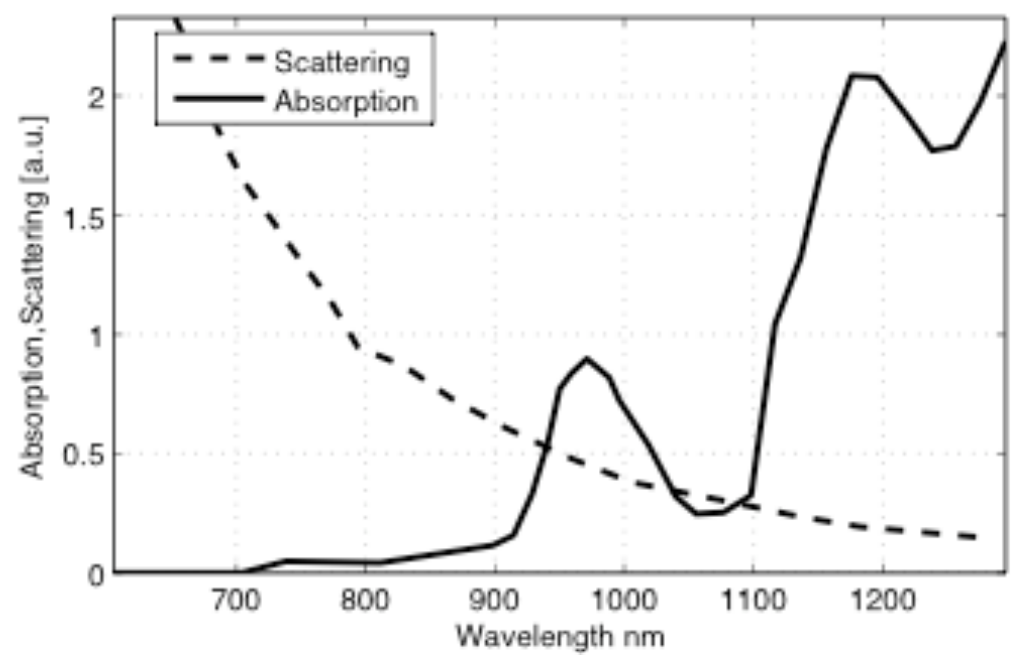

Figure 1-2: Scattering and absorption peaks of water [3].

OCT utilizes near infrared wavelengths of $1310 \mathrm{~nm}$ or $800 \mathrm{~nm}$ for interferometry purposes due to its increased depth penetration at longer wavelengths. OCT light sources that are centred at $1060 \mathrm{~nm}$ wavelengths have also been developed due to the optical properties of water as biological tissues contain large amounts of water. Therefore, the absorption and scattering of light in water is key to understanding and optimizing OCT imaging. The absorption and scattering of broadband light in water is depicted in Figure 1-2. The resulting imaging wavelength chosen must have a maximized scattering peak, a minimized absorption peak, and maximized depth penetration [3-4].

OCT has had a major impact on preclinical and clinical research, transforming ophthalmology, intravascular coronary imaging, and have an impact in other areas of medicine including neurosurgery, oncology, radiology, bronchoscopy, gastrointestinal endoscopy, laparoscopic surgery, urology, gynecology, plastic surgery and dermatology. In ophthalmology, OCT has been used to study retinal pathology [5]. Catheters and probes have been developed for imaging the microvasculature and microstructures [6-8]. 
Intravascular OCT has also been used in studies of the gastrointestinal tract and in vivo vessel imaging [9-10].

There are also applications of OCT (Doppler OCT, speckle variance, phase variance, etc.) that are specifically geared for visualizing microvasculature and other targets to provide additional clinical information. OCT is a powerful imaging technique that can be used for microstructural studies with many clinical benefits. The continuation of OCT research will only develop and enhance the clinical usage of OCT instrumentation in medical imaging.

The goal of this thesis is to develop and implement a method for the analysis of in vivo mouse tumour speckle decorrelation time maps obtained from an OCT adaptation of dynamic light scattering (DLS). It was previously shown that the cellular and intracellular motion of cells undergoing apoptosis (a known form of cell death) could be monitored using dynamic light scattering with OCT [11]. After the in vitro studies were completed, speckle decorrelation time (DT) maps were generated from the DLS-OCT images in vivo [12]. In the preliminary study involving three mice, the authors measured variations in the speckle DT maps between tumour regions and the surrounding non-tumour tissue. In addition, changes in the tissue DT were also observed, in the same tumour regions, as a function of treatment time for mice treated with a chemotherapy drug. The study, however, had several limitations: apart from the small number of animals used, the DT maps had regions of interest (ROI) that included contributions to the DT maps from blood vessels, not just from regions of tumour cells. As the DT maps that enclosed only cells responding to the chemotherapeutic treatment was of interest, a method to confine the analysis to such regions was needed. Therefore, the motivation of this thesis was to: 
a) establish the repeatability of the previous results with a larger in vivo dataset, and b) introduce a segmentation technique to remove any DT contributions from blood flow. Extensions to this thesis include protocol refinements for future in vivo experiments for utilizing DT measurements of intracellular motion as an endogenous contrast method for tumour-related studies.

\subsection{Thesis Objectives}

The first objective of this thesis is to implement a speckle decorrelation time analysis algorithm for an $n=10$ mouse tumour model study, building upon the previous $\mathrm{n}=3$ feasibility test performed by Farhat et al. [12]. This is done to demonstrate the repeatability of the protocol and to obtain better statistical significance for the comparisons of the DT maps for mice undergoing cisplatin treatment.

The second objective of this thesis is to confine the DT analysis to regions in vivo that do not include bulk flow due to blood motions. To achieve this, the microvasculature needs to be segmented and removed from the regions used in the analysis. The previous study contained DT results that took into account the DT contributions from blood vessel locations, which may dominate the OCT DT signals. A method needs to be developed to threshold DT data so that only areas without the bulk flow of blood are used in the DT analysis of the tissues.

\subsection{Thesis Hypothesis}


Speckle decorrelation time (DT) analysis of mouse tumours can be performed reproducibly in vivo and the application of thresholds to segment out DT contributions from blood vessels will allow a more reliable method of detecting cell death in vivo. 


\section{Chapter 2: Background}

\subsection{Dynamic Light Scattering}

The heterogeneity and dynamicity of cellular tissue are utilized in light scattering tissue imaging. The organelles of living cells provide the dynamic character that can be probed by light scattering techniques. It is thought that the active functions of the cytoskeleton [13-14] and molecular motors [15] that drive the transport systems in the cytosol [16] produce fluctuations in the scattered light detected.

Dynamic light scattering (DLS) is the use of light scattering techniques for detecting and measuring the dynamic character of a target. DLS in tissues can be performed as quasi-elastic light scattering (QELS) when predominantly singly-scattered light is used, whereas diffusing-wave spectroscopy (DWS) or diffusing correlation spectroscopy (DCS) is used for light that is multiply scattered [13-21]. Previous QELS research includes the study of dynamics in the nucleus [17], cytosol [18], and membrane fluctuations [19]. Previous DWS and DCS research includes the study of actin filament networks [20] and imaging dynamic heterogeneities [21]. Speckle contrast of blood flow has also been studied using DLS techniques. This thesis utilizes some of these concepts to observe the tissue dynamics by measuring speckle decorrelation time of the tissue.

\subsubsection{Principle}

DLS involves the analysis of light intensity fluctuations of the sample refractive index $(\delta n(r, t))$ recorded from the sample scatters [17]. The sample refractive index is dynamic due to the changing density, concentration, and composition of the tissue light 
scattering structures [22]. The scattered light is proportional to the spatial Fourier transform of the sample refractive index: $\delta^{\wedge} n(\mathbf{q}, t)$. In this equation, $\mathbf{q}$ is the scattering wave vector that defines the characteristic length $(l)$ on which the fluctuations of the refractive index take place $(l=2 \pi / \mathrm{q} ; \mathrm{q}$ being the modulus of $\mathbf{q})$. For elastic scattering, the photon energy is conserved and $q=(4 \pi n / \lambda) \sin (\theta / 2)$, where $n=$ sample refractive index; $\lambda$ $=$ wavelength; and $\theta=$ scattering angle (between the incoming direction and the scattering direction). The fluctuations of the scattered light (related to the sample refractive index) is measured and analyzed to obtain the sample dynamics of the tissue.

\subsection{Optical Coherence Tomography}

Optical coherence tomography (OCT) is an imaging modality that utilizes photonic devices for near-histological resolution imaging. OCT implements near infrared light interferometry to detect backscattered waves, and have been applied clinically for non-invasive or minimally invasive imaging of tissues. OCT is most often compared to ultrasound (US) due to the analogous concept of sending incident signals and detecting the backscattered response. In US, a sound wave is propagated from a transducer and interrogates the tissue sample. The transducer records the backreflected signals from the sample, and the resulting image is formed. In OCT, the concept is similarly utilized, except light waves are used instead of sound waves, and the respective system components are suited for light imaging. Direct contact between the wave source and the imaged sample is also unnecessary in OCT.

OCT is known for its excellent spatial resolution due to the wavelength of light in the infrared range ( $\sim 1300 \mathrm{~nm})$, compared to US wavelengths (in the millimetre range for 
ultrasound frequencies between 1-5 MHz). The drawback for OCT is the limited penetration depth of 1-3 $\mathrm{mm}$, which differs from US penetration of greater than $10 \mathrm{~mm}$, depending on the ultrasound frequencies used.

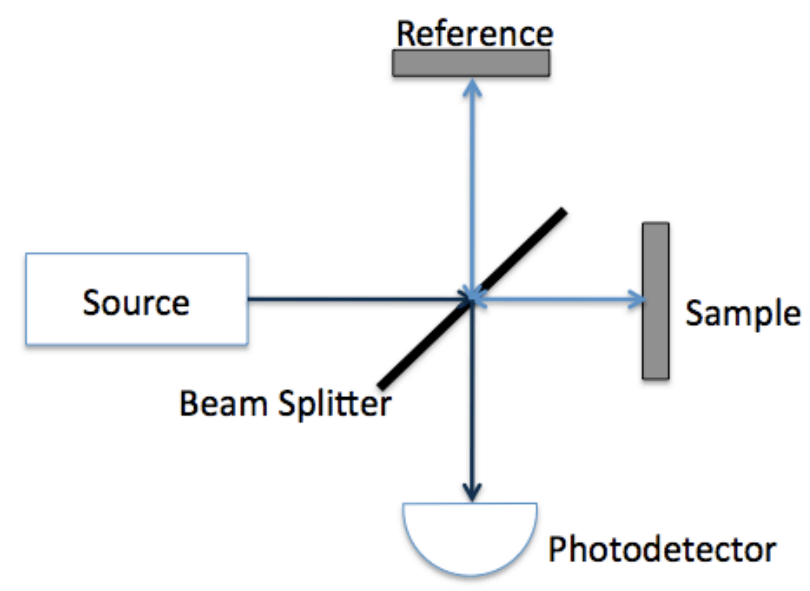

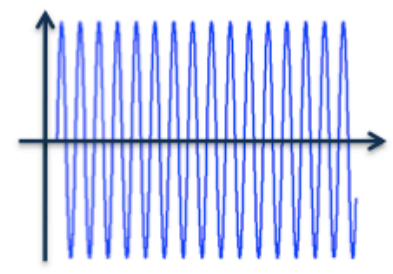

Long Coherence Length

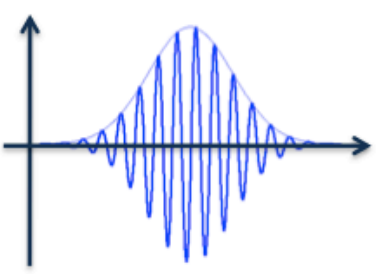

Short Coherence Length

Figure 2-1: (Top) Michelson interferometer; (Bottom) Depiction of long and short coherence lengths [2].

\subsubsection{Interferometry}

Because OCT uses light that travels at the speed of $\sim 3 \times 10^{8} \mathrm{~m} / \mathrm{s}$, existing electronic equipment are not suitable for formulating the resulting image from the time delay of the backscattered light. OCT uses interferometry techniques to gate the data acquisition to produce depth resolved reflectivity profiles of the imaging target.

Figure 2-1 depicts a Michelson interferometer setup, a common configuration for optical interferometry. The light source emits the light towards the beam splitter and is 
represented by $E_{t}(t)=E_{i} \cos (\omega t-k z)$. In this representation, $E_{t}$ is the electric field at the position $z$ at time $t, k$ is the wave number given by $2 \pi / \lambda$, where $\lambda$ is the wavelength and $\omega$ is the angular frequency. A beam splitter then divides this beam of light and directs the two beams to the reference mirror and sample, respectively. These two paths are denoted by $E_{r}(t)$ and $E_{s}(t)$ to represent the reference path and sample path, respectively, where $E_{t}(t)=E_{r}(t)+E_{s}(t)$. After the two beams reflect off of the reference mirror and sample, they recombine at the beam splitter. The interferometer output observed at the photodetector is $\sim E_{r}(t)+E_{s}(t)$. The intensity of the output beam is measured by this detector, and is also proportional to the square of the electric field. The intensity at the detector is:

$I_{0}(t) \sim \frac{1}{4}\left|E_{r}\right|^{2}+\frac{1}{4}\left|E_{S}\right|^{2}+\frac{1}{2} E_{r} E_{S} \cos \left(2 \frac{2 \pi}{\lambda} \Delta l\right)$

where $\Delta l$ is the path difference between the reference arm length and the sample arm length ( $l_{r}$ and $l_{s}$ respectively).

It can be seen from Eq.2-1 that if the path difference $\Delta l$ varies, the output intensity $I_{o}(t)$ will vary. This is depicted in the lower two graphs in Figure 2-1, comparing long and short coherence lengths of the light source. A long coherence length refers to a monochromatic light source; a light source with a short coherence length is broadband. The coherence length is inversely proportional to the bandwidth, and thus, for low coherence light sources, interference can only be observed when the path difference between the reference and sample lengths is less than the coherence length [23]. 

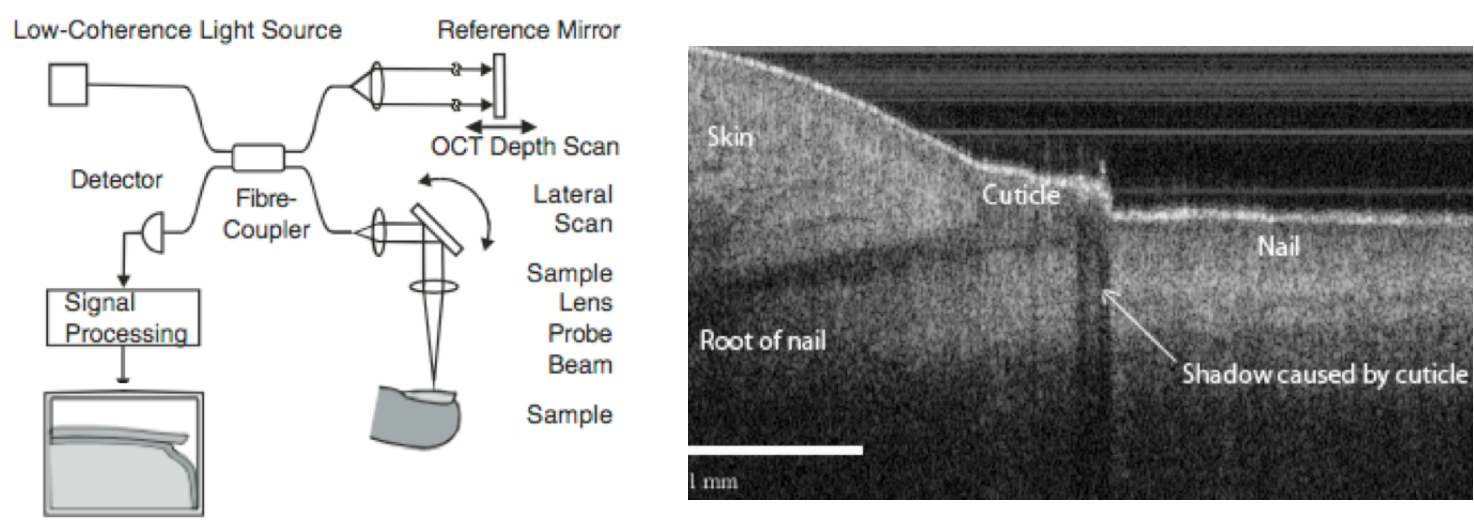

Figure 2-2: (Left) Time-domain OCT (TD-OCT) setup [24]; (Right) OCT image of a fingernail [25].

Figure 2-2 shows the Michelson interferometer as part of a time-domain OCT (TD-OCT) system. The differences here include a fibre coupler in place of the free space beam splitter, a translatable reference mirror for depth adjusted scanning, and additional scanning mirror and focusing lens for raster scanning of the sample. Other OCT enhancements for current OCT system setups will be described later in this chapter.

\subsubsection{Resolution}

The resolution of an imaging system refers to its capabilities for resolving the details of a target. The resolution specifications are vital to the quality of the image. For OCT imaging, the axial and transverse (lateral) resolutions are independent of each other, which differ from confocal microscopy.

The transverse (lateral) resolution of an OCT system is dependent on the imaging optics that forms the sample arm. For example, the focusing lens of the sample arm will have a specific focal length that affects the transverse resolution of the system. Also, the 
beam spot size and wavelength used affects the overall minimum spot size (transverse resolution). Eq.2-2 depicts the equation to calculate this minimum spot size:

$\Delta x=\frac{4 \lambda}{\pi}\left(\frac{f}{d}\right)$

where $\lambda$ and $d$ are the wavelength and spot size of the incident beam, respectively, and $f$ is the lens focal length. Note that the ratio $(f / d)$ is also referred to as the numerical aperture (NA) of the optical system. Eq.2-2 shows that in order to obtain high transverse resolution, a high NA is required by focusing the incident beam to a small spot size. This, however, will reduce the depth of focus $(b)$, which is proportional to the transverse resolution $\Delta x$ [2]. Eq.2-3 describes the relation between the depth of focus and the transverse resolution. Figure 2-3 depicts the difference between a low NA and high NA setup, demonstrating the trade-off for transverse resolution and depth of focus. The depth of focus is given by:

$b=\frac{\pi \Delta x^{2}}{2 \lambda}$
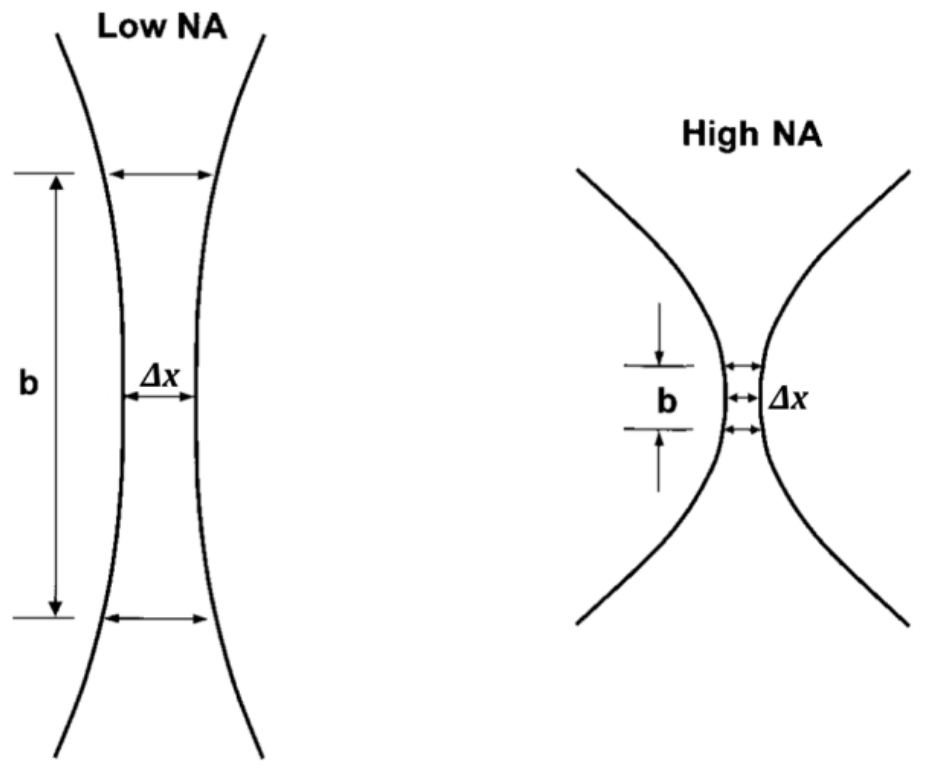

Figure 2-3: The difference between using a low NA and high NA lens, due to the tradeoff between the depth of focus (b) and the transverse resolution $(\Delta x)$ [2]. 
Therefore, careful selection of the imaging optics is important to obtain the desired depth of focus and transverse resolution. The focusing lens comes in varying NAs that should be chosen based on the intended imaging target. Typically for microvasculature imaging, a lens with higher NA is desired for better transverse resolution when imaging target vessels in the micron range.

The coherence length of the OCT light source used determines the axial resolution of the imaging system. Because there is no reliance on the sample arm focusing optics, the axial resolution is independent of the transverse resolution. The coherence length $(\Delta z)$ is shown in Eq.2-4.

$\Delta z=2 \ln (2) \frac{\lambda_{c}{ }^{2}}{\pi \Delta \lambda}$

where $\lambda_{c}$ is the centre wavelength of the broadband light source and $\Delta \lambda$ is the bandwidth of the light source at full-width half-maximum (FWHM). Therefore, the axial resolution can be improved by using a shorter centre wavelength and a wider bandwidth [1].

\subsubsection{Fourier-Domain OCT}

The previous description of the Michelson interferometer in Section 2.2.1 involves a time-domain OCT setup. In TD-OCT, a beam splitter evenly divides half of the light from a light source towards the sample, and half to a reference mirror. The light reflected from both the sample and reference mirror are combined to the beam splitter before reaching the detector [26]. Cross-sectional information across the axial line through the sample is called an A-scan and is acquired by scanning the reference arm position axially. In Fourier-domain OCT (FD-OCT), the reference mirror does not move, 
but is instead replaced by either a charged coupled device (CCD) for spectral-domain OCT (SD-OCT) or a wavelength tuning light source for swept-source OCT (SS-OCT). Rather, the desired depth information is encoded in the spectrum of the processed interferogram. Performing a Fourier transform to this spectrum will provide a backreflection profile as a function of depth. Because FD-OCT does not require a translating reference mirror, the image acquisition speed is significantly increased.

The method for resolving depth without the need for a translating reference mirror is described. Eq.2-5 describes the backscattered light $\mathrm{U}$ at depth $\mathrm{z}$.

$U(z)=U_{0} e^{-i k_{0} n z}$

$U_{z}=U_{0} e^{-i k_{0} n z}$

where $U_{0}$ is the amplitude, $k_{0}=2 \pi / \lambda_{0}$ is the wave number, $\lambda_{0}$ is the wavelength, and $n$ is the index of refraction. For a monochromatic light source, the interference at the photodetector can be represented by $I_{\Delta z}\left(k_{0}\right)$ and is described in Eq.2-6.

$I_{\Delta z}\left(k_{0}\right)=\left|U_{r}+U_{s}\right|^{2}$

$I_{\Delta z}\left(k_{0}\right)=\left|U_{0}\left(e^{-i k_{0} n z}+e^{-i k_{0} n(z+2 \Delta z)}\right)\right|^{2}$

$I_{\Delta z}\left(k_{0}\right)=U_{0}^{2}\left(e^{-i k_{0} n z}+e^{-i k_{0} n(z+2 \Delta z)}\right)\left(e^{i k_{0} n z}+e^{i k_{0} n(z+2 \Delta z)}\right)$

Eq.2-6

$I_{\Delta z}\left(k_{0}\right)=U_{0}^{2}\left(2+e^{i k_{0} n 2 \Delta z}+e^{-i k_{0} n 2 \Delta z}\right)$

$I_{\Delta z}\left(k_{0}\right)=U_{0}^{2}\left(1+\cos \left(k_{0} 2 \Delta z\right)\right)$

where $U_{r}$ and $U_{s}$ are the reference arm and sample arm light, respectively. They are both equal in magnitude. Note that within the cosine, the $2 \Delta z$ represents the path difference between the reference and sample arms. Due to the depth dependency of the reflectivity profile, $a(z)$ can represent the reflectivity at any $\mathrm{z}$ position, and therefore, $U_{r}$ can be 
substituted with $U_{0} a(z)$. The intensity of the interference is now represented in Eq.2-7 and Eq.2-8 for a monochromatic and broadband light source, respectively.

$I\left(k_{0}\right)=2 I_{0}\left(1+\int_{0}^{\infty} a(z) \cos \left(2 k_{0} n z\right) d z\right)$

$I(k)=2 I_{0}\left(1+\int_{0}^{\infty} a(z) \cos (2 k n z) d z\right)$

Here, the depth information of the OCT signal is encoded in the cosine argument. Using the Fourier transform, the intensity profile can be resolved based on depth as shown in Eq.2-9.

$I(z)=F T\left\{2 I_{0}\left(1+\int_{0}^{\infty} a(z) \cos \left(2 k_{0} n z\right) d z\right)\right\}$

\subsubsection{Spectral-Domain OCT}

SD-OCT is a form of FD-OCT that uses a wide bandwidth super-luminescent diode (SLD) for its light source. SD-OCT obtains spectral information by separating the wavelengths onto a detector stripe to determine the interference spectrum, such as a highspeed CCD detector array, via a grating [26]. The information for the full depth scan can be acquired after performing a Fourier transform, but the low dynamic range of each stripe detector (compared to a single detector) negates the overall signal-to-noise ratio (SNR) advantage of FD-OCT over TD-OCT. Another drawback to SD-OCT is the dispersive elements do not separate the light evenly in wavelength on the detector, requiring signal resampling before processing, thus reducing image quality. Also, the discharge time of an individual CCD detector limits the overall imaging speed of the system.

\subsubsection{Swept-Source OCT}


The spectral components of the signal for SS-OCT are not encoded by spatial separation, but encoded in time [26]. SS-OCT utilizes a laser source of small filtered linewidths swept linearly in wavelength over time, and uses a high-speed photodetector instead of the CCD in SD-OCT. These two components of SS-OCT in combination create a time resolved spectrometer. Because the photodetector recognizes photons regardless of the wavelength, data acquisition time is minimized in comparison to the discharge time for the $\mathrm{CCD}$ in SD-OCT. Each wavelength sweep through the entire sweep range (spectral bandwidth) corresponds to a single axial scan. A Fourier transformation of the resulting signal is performed to reconstruct the signal information. The advantages of using SS-OCT are keeping the high SNR detection and achieving small linewidths at high frequencies to produce quality OCT images.

\subsubsection{OCT Research Applications}

There are many applications of OCT in the health and biomedical fields due to its advantage in detection sensitivity and fast imaging speed [27]. Even though OCT is limited to millimetre penetration depth, OCT has become an established imaging modality for beneath skin imaging.

\subsubsection{OCT in Ophthalmology}

Retinal OCT has been a mainstay in OCT applications due to the high transmission and low scattering regions of the eye - effective conditions for OCT imaging. OCT can provide high depth resolution at a low NA for larger depth of focus, which is ideal for retinal imaging. The development of ultrabroad-bandwidth light 
sources has enabled ultrahigh-resolution (UHR) OCT techniques for studies such as the direct correlation of photoreceptor loss with retinal function in Stargardt's dystrophy [28] and therapy monitoring for eye disease pathogenesis [29-31].

2D retinal imaging of multiple B-scans have also advanced into $3 \mathrm{D}$ retinal imaging to provide improved volumetric structural information for disease assessment, especially for in vivo imaging. FD-OCT techniques provide the high-speed requirement vital for 3D in vivo imaging in virtual biopsy and surgery. The rotation of the 3D volume has also enabled "fly through" features to obtain further position and orientation data for diagnostic needs [32]. Examples of 3D retinal imaging studies include 3D volume optic nerve and skin imaging constructed from 2D images at different depths [33], as well as 3D UHR OCT images with SD-OCT A-scans [34].

High definition 3D images of the normal retina and optic nerve head have been obtained by an ultrahigh speed SS-OCT retinal imaging system at 249,000 A-scan per second at $1060 \mathrm{~nm}$ wavelength [35]. This imaging system allowed increased light penetration to view the choroid, lamina cribrosa, and sclera. En face images were also acquired to visualize the nerve fibre layer, photoreceptors, and even capillaries of the retina. The high resolution and speed capabilities of SS-OCT are clear advantages that are maximized with this imaging modality for specific ophthalmologic studies.

\subsubsection{Intravascular OCT}

Non-invasive imaging techniques for cardiovascular studies have been challenging due to the small diameter and tortuosity of microvasculature [27]. The use of 
optical probes and catheters has enabled OCT intravascular imaging of blood vessels for various cardiovascular studies, overcoming those challenges.
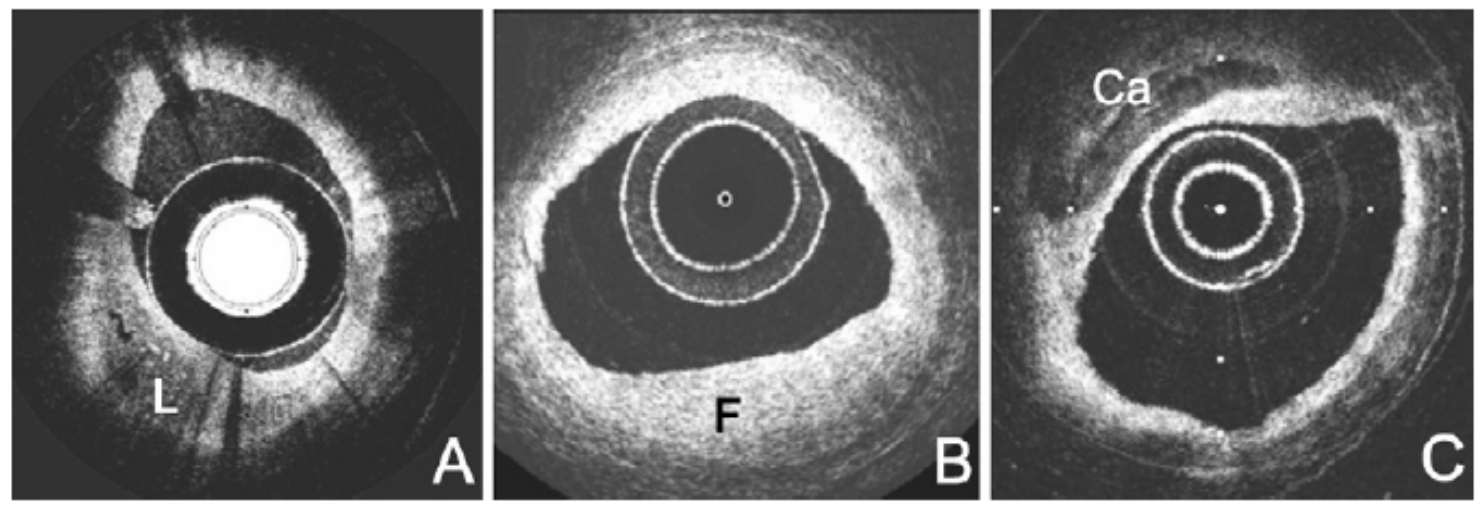

Figure 2-4: TD-OCT images at $\lambda=1300 \mathrm{~nm}$ of lipid-rich (A), fibrous (B), and calcific (C) coronary atherosclerotic plaque types. Lipid-rich $(\mathrm{L})$ and calcific $(\mathrm{Ca})$ regions appear as a signal-poor region within the vessel wall. The OCT signal of fibrous plaque $(\mathrm{F})$ is observed to be strong and homogeneous [27].

Fibre optics using low coherence interferometry has been utilized for atherosclerosis imaging. In vivo images of atherosclerotic plaque types (Figure 2-4) have been obtained and are comparable in features to ex vivo gold standard atherosclerosis plaque characterizing studies. The in vivo results were also consistent with results from intravascular ultrasound (IVUS) - the benchmark for intracoronary imaging [36-38]. Intravascular OCT is also used for detecting atherosclerotic lesions, including features such as vessel wall appearance, subintimal area reflectivity, and internal elastic membrane discontinuation [39]. Plaque rupture has also been studied and OCT images showed the morphology for the ruptured plaque analyzed [40].

Statin research and stent implantation are two other research areas where intravascular OCT has provided great adaptations to previous approaches. Intravascular OCT has aided statin therapy, a process to lower lipid concentration, by providing images 
of the fibrous cap thickness. This measure is vital to the assessment of statin levels for patients that require lipid concentration monitoring [41]. Stent implantation is used to avoid arterial reocclusion. OCT has already been used to image in vivo to help prototype the appropriate stent strut for the vessel wall. IVUS cannot detect neointimal layers for the sirolimus eluting stents, thus OCT is a suitable choice for visualization thin neointima of each stent strut. [42]

\subsubsection{OCT in Oncology}

Early detection and assessment of malignant neoplasm is vital to the clinician in providing the appropriate patient treatment and care. OCT is an imaging modality that can provide optical biopsies at high resolutions comparable to histopathology without the need for tissue removal. This capability will allow clinicians to better identify malignancies at curable stages. OCT is also involved in aiding surgical resection, radiation therapy, and analyzing tumour death and tissue pathology [26].

OCT presents its greatest potential in the imaging of epithelial cancers due to the high quality imaging of epithelial structures for identifying cancers at an early stage. OCT is able to differentiate the tissue layering and assess cancer growth by observing surface specialization (cilia or keratin), cell shape and layers. Additional OCT techniques such as speckle variance analysis, polarization sensitivity, and elastography are also applied to better analyze these factors in OCT imaging due to the lack of contrast in identifying these factors [26].

The study of cancers via OCT imaging has provided clinicians with valuable information in disease analysis. OCT has been used to image tissues with esophageal, 
bladder, lung, breast, colon, skin, and many other cancers. OCT can adequately detect the presence and absence of markers such as calcium deposits and also differentiate glandular structures and tissue boundaries, basement membranes, and cell morphologies. These markers are determined after comparing the processed OCT images (taken in real time) with the corresponding histology [26].

\subsection{Detecting Apoptosis}

The detection of apoptosis will allow better analysis of tissue development and tumour responses to anticancer drugs. Real-time non-invasive apoptosis measurements have been performed to observe the kinetics and methods for inducing apoptosis. These apoptotic tissue regions produce increased ultrasound backscatter that can be detected and differentiated from non-apoptotic regions. The monitoring of these tissues has led to studies of tissue response to treatments for indications of ex vivo and in vivo programmed cell death [43]. Figure 2-5 depicts the result of ultrasound imaging of apoptotic cells and shows the change in scattering as a function of exposure time (which is related to the number of cells responding to the treatment).

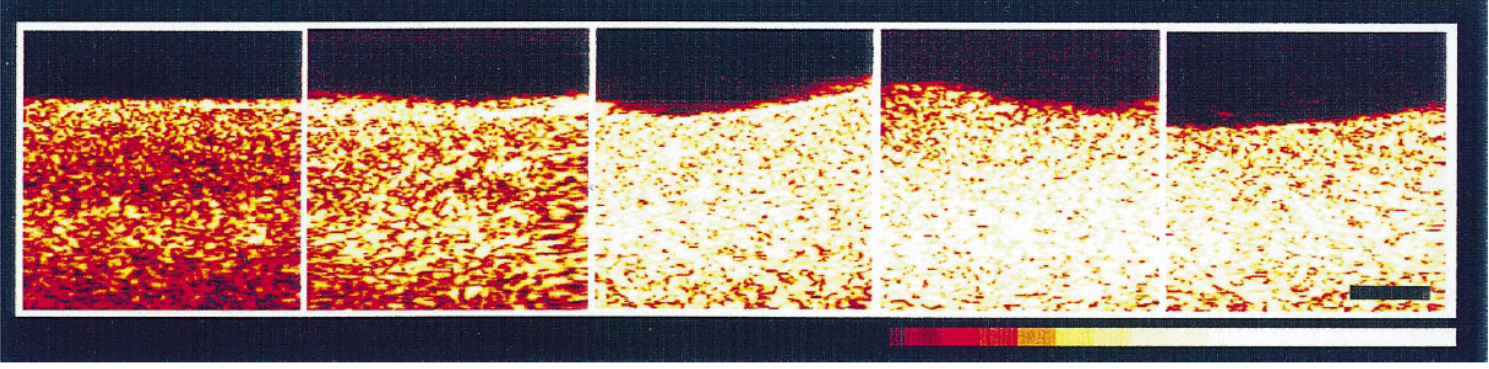

Figure 2-5: Results of $40 \mathrm{MHz}$ ultrasound imaging of centrifuged apoptotic cells. Each panel refers to a representative ultrasound scan of a pellet of acute myeloid leukemia (AML) cells. From left to right, panels correspond to cells treated with cisplatinum for 0 , $6,12,24$ and $48 \mathrm{~h}$ to induce varying degrees of apoptosis. The scale bar indicates $1 \mathrm{~mm}$ [43]. 


\subsubsection{Principle}

In OCT, speckle intensity measurements are a tool for assessing different tissue types [11]. The speckle can change due to the size, number, and optical properties of the scatterers in the medium imaged. The speckle occurs in living tissues due to the fluctuations of subresolution scatterers, including those caused by the intracellular motion from mitosis, organelles movements, and other cellular morphological changes in connection with apoptosis. Apoptosis involves the programmable structural and biokinetic changes that ultimately results in cell death [44]. An apoptotic cell exhibits shrinking, nuclear fragmentation, membrane blebbing, and organelle disintegration. These responses are of interest in cancer therapies where the chemotherapeutic drug will induce cancer cells to undergo apoptosis in order eradicate the tumour [11].

\subsubsection{Apoptosis Detection Using OCT}

Dynamic light scattering has been adapted for OCT to measure intracellular motion (IM) for detecting and differentiating between viable and apoptotic cells due to changes in the rate of IM, in an in vitro setting [11]. The decorrelation rate of speckle for these cisplatin-treated acute myeloid leukemia (AML) cells undergoing apoptosis was measured from multiple consecutive SS-OCT images. This technique incorporated the "heterodyne approach" in removing contributions from static scattering components [45], to produce an average decorrelation time (DT) used as the overall measurement of IM. The DT is measured by the half width of the intermediate scattering function (calculated from the intensity autocorrelation function) at half its maximum value and describes how 
quickly the speckle intensity changes. Alternatively, the time it takes for the intermediate scattering function to fall below 1/e can also be used as the measure of the DT. The cell morphologies over time, assessed by H\&E histology, indicated membrane blebbing and fragmentation, which, in combination with the decreasing DT, was used to explain the decrease in DT (thus increase in IM) caused by the cell structural changes during apoptotic cell death [11]. Figure 2-6 depicts the histology and signal intensity curves of a single pixel in the OCT image of cisplatin treated cells over time. Figure 2-7 shows the decrease of DT over time.

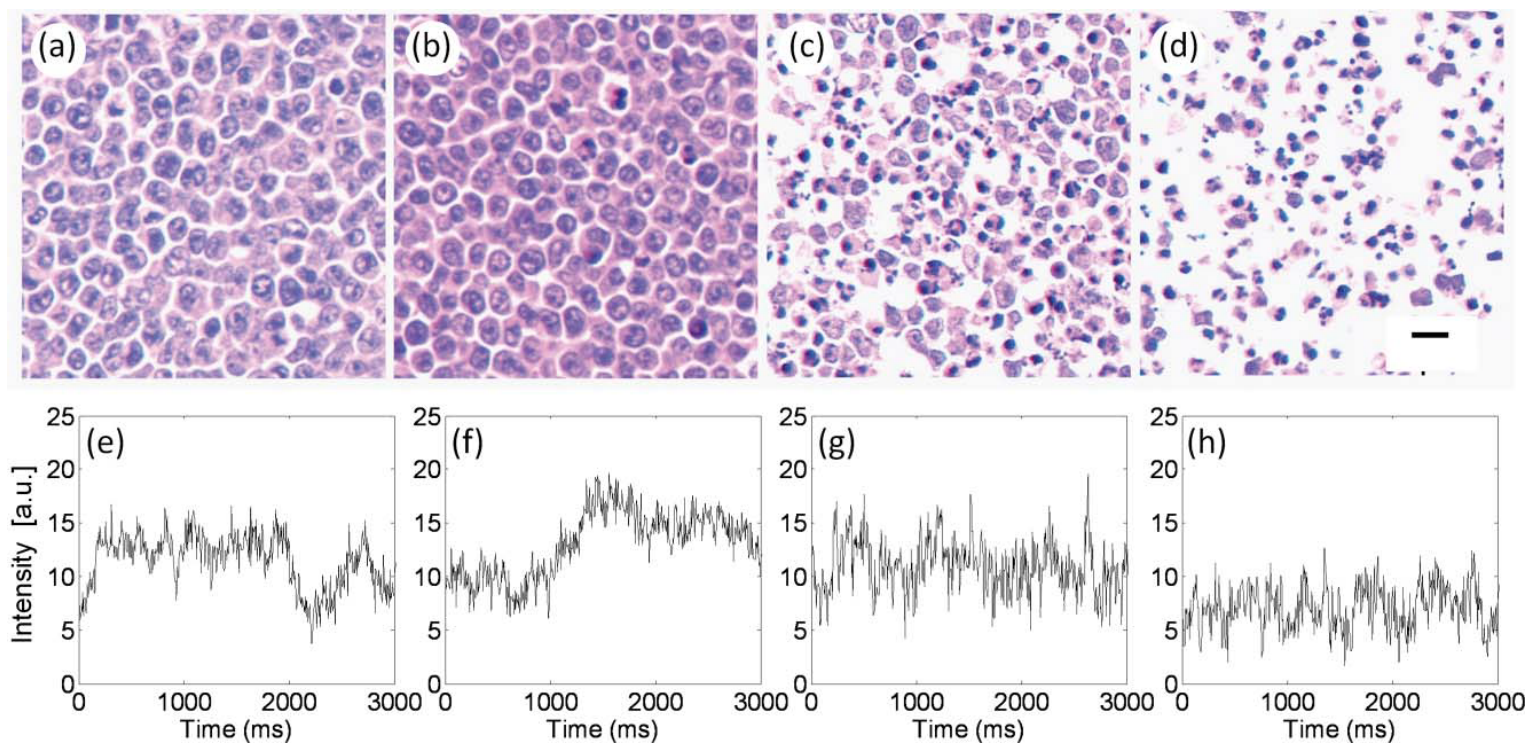

Figure 2-6: Hematoxylin and eosin (H\&E) stained sections obtained from cisplatin treated cells after (a) $0 \mathrm{~h}$, (b) $12 \mathrm{~h}$, (c) $24 \mathrm{~h}$, and (d) $48 \mathrm{~h}$ of treatment. The scale bar represents $10 \mu \mathrm{m}$. Representative signal intensity curves from a single pixel at (e) $0 \mathrm{~h}$, (f) $12 \mathrm{~h},(\mathrm{~g}) 24 \mathrm{~h}$, and (h) $48 \mathrm{~h} \mathrm{[11].}$ 

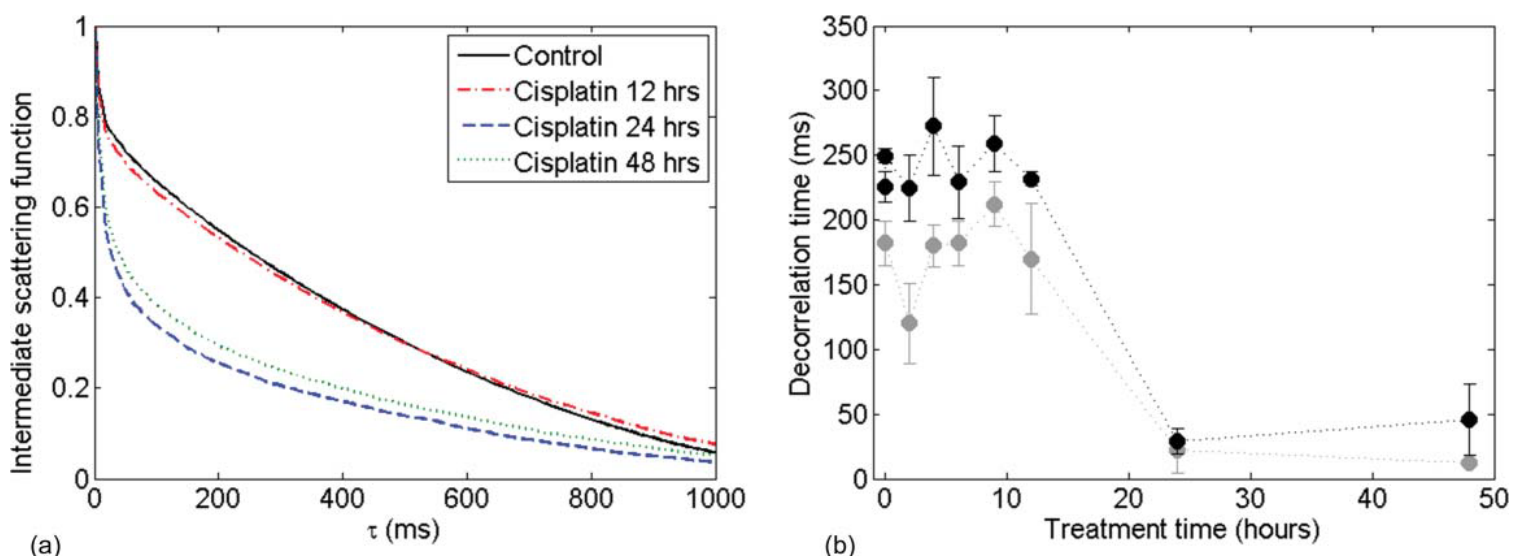

Figure 2-7: (a) Average intermediate scattering functions computed from a selected ROI in AML cell pellets. (b) Decorrelation time computed from AML cell samples treated with cisplatin over a $48 \mathrm{~h}$ period. Each curve corresponds to a separate experiment. Error bars represent the standard deviation of 10 separate measurements from each sample [11].

This in vitro test has shown the technique's sensitivity to IM variations, which is correlated to cell death. The DLS technique applied to OCT utilizes signal fluctuations in the DT calculations instead of absolute value of signal intensity, thus minimizing the effects of signal attenuation and scattering angle on the measured signal, which makes this technique advantageous over other methods that measure OCT backscatter strength or attenuation to detect cell death [46]. The drawback for this method is the increased sensitivity to rapid bulk tissue motion and in vivo blood flow. Therefore, an image processing technique for vascular segmentation is required to correct the DT maps produced in the in vivo setting.

The DLS-OCT technique has been implemented in vivo for cell death detection in a mouse tumour model for feasibility testing [12]. Human bladder carcinoma tumours were grown on three nude mice and imaged with OCT. DT maps were generated for tumour and skin regions of the mice before and after chemotherapy treatment. Figure 2-8 
shows the tumour and skin regions on the OCT image, with the respective DT map and autocorrelation curves.
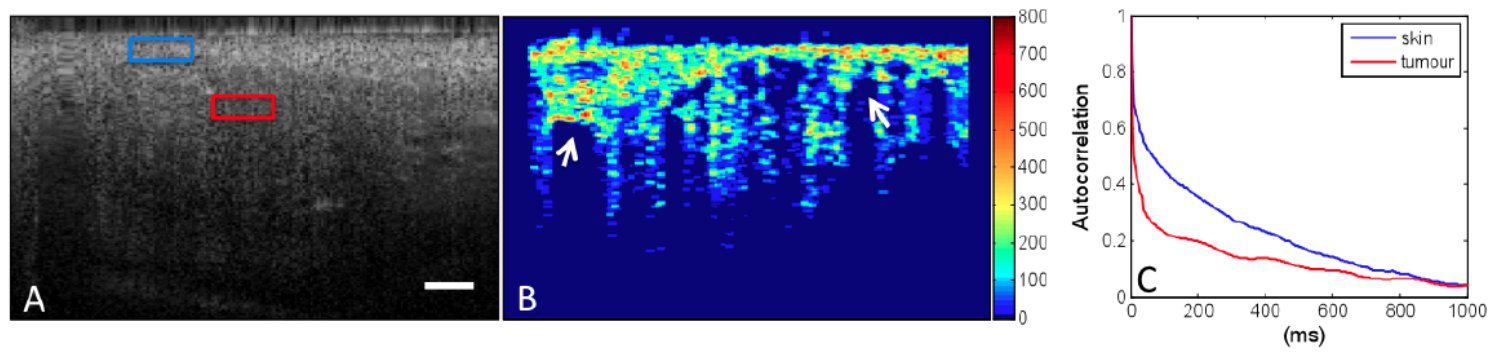

Figure 2-8: Comparing decorrelation in skin versus tumour tissue. OCT b-mode image (A), decorrelation map (B) and average autocorrelation functions obtained from regions of skin and tumour highlighted in A (C). White arrows point to regions of blood flow represented by very short DT. Scale bar in the OCT image is $300 \mu \mathrm{m}$ and the decorrelation times in ms are indicated by the colour bar [12].

DT differences were observed over time, including the DT increase at 24 and 48 hours for the in vivo mouse tumours [12], which differed from the in vitro data where DT decreased over time [11]. These preliminary results showed the feasibility of DLS-OCT for IM measurements, but required further experimental analysis to confirm the previous in vivo results with stronger statistical significance. This thesis provided further analysis in a larger $\mathrm{n}=10$ mice study that built on this initial in vivo feasibility test [12]. 


\section{Chapter 3: Experimental Methods}

The experimental methods for this thesis include the setup of: (a) the imaging system and (b) the design and implementation of the animal experiment. A swept-source optical coherence tomography (SS-OCT) system was used for the in vivo mouse tumour imaging. The animal experimental setup involved the preparation of the tumour cell line, nude mice, window chamber surgeries and chemotherapy drug. Methods were also developed for the data acquisition and analysis, as well as the histology staining. Figure 3-1 is an example set of histology images, OCT b-mode images and decorrelation time maps for a previous DLS-OCT study with a similar methodology to this thesis [12].

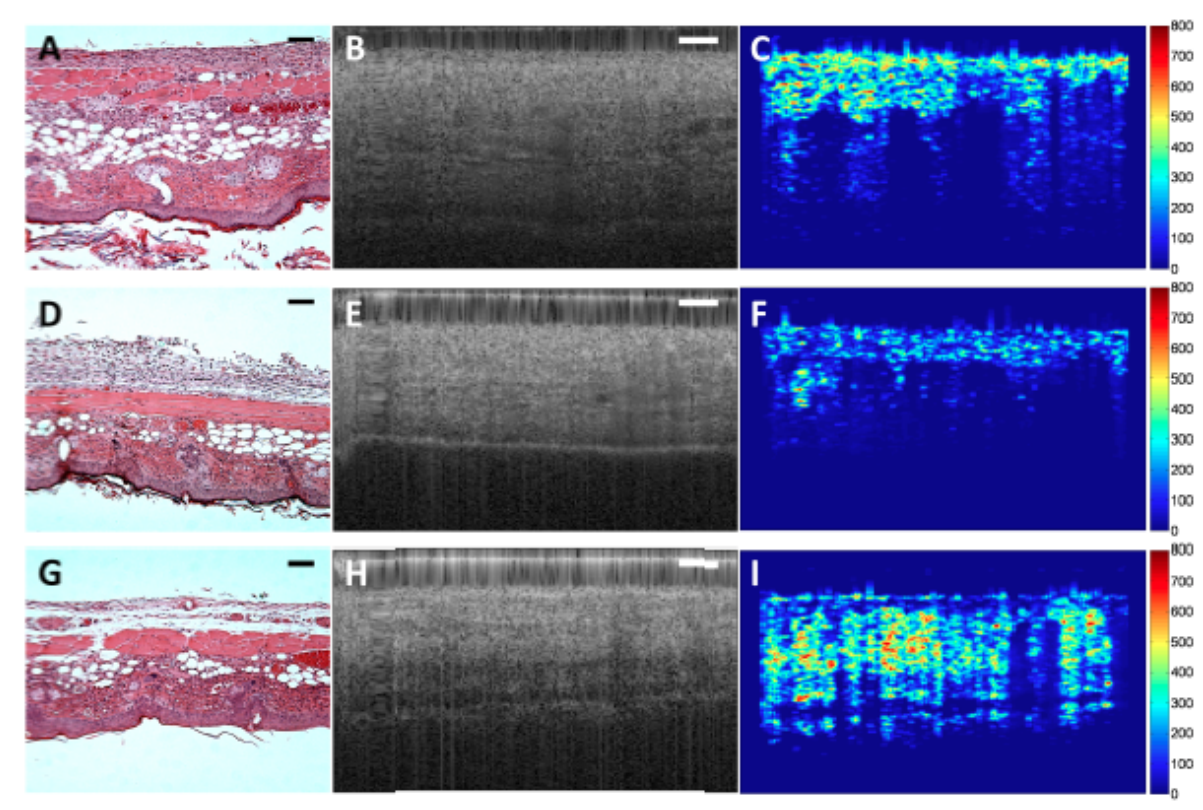

Figure 3-1: Variations in skin structure. The left column shows H\&E stained sections of three mice. The middle column shows the corresponding OCT b-mode images. The right column shows decorrelation time maps processed from the OCT $b$-mode images. Scale bars are $50 \mu \mathrm{m}$ (histology) and $300 \mu \mathrm{m}$ (OCT and decorrelation time maps).

Decorrelation times are in milliseconds [12]. 


\subsection{Imaging System Setup}

A SS-OCT system was used for all of the in vivo experiments for this thesis. This

system was designed and housed at the Princess Margaret Hospital on the $7^{\text {th }}$ floor Wilson Lab. The members of the Biophotonics and Bioengineering Lab at Ryerson University and the Vitkin and Wilson Labs at Ontario Cancer Institute (University Health Network) and University of Toronto constructed this system based on the design of the Harvard Medical School and Wellman Laboratories for Photomedicine at Massachusetts General Hospital in Boston, Massachusetts, USA [47].

\subsubsection{Imaging System Rationale}

A dynamic light scattering-optical coherence tomography (DLS-OCT) imaging system was designed to measure subtle differences in motion related to cell viability. A SS-OCT setup was used due to its high speed and SNR. A DLS-OCT setup had been used previously to measure in vitro changes in intracellular motion caused by apoptosis in acute myeloid leukemia cells treated with cisplatin [11]. The SS-OCT system had been used in previous in vivo mouse studies involving the imaging of tumour network microvasculature [48].

\subsubsection{Imaging System Specifications}




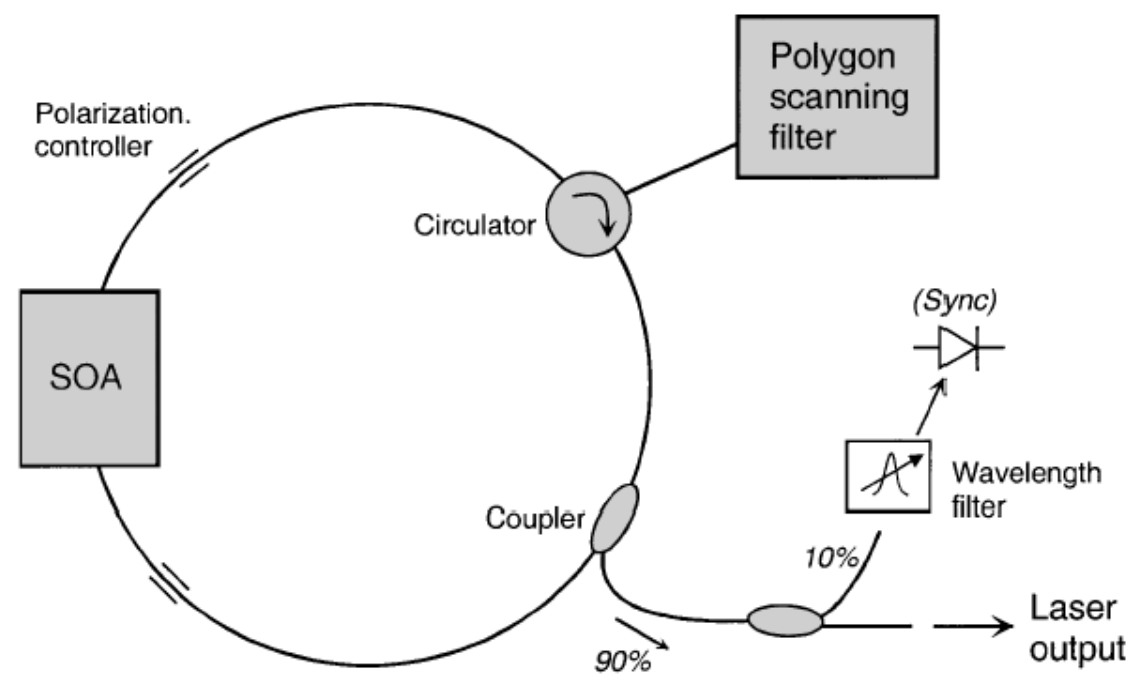

Figure 3-2: Schematic for the high-speed wavelength-swept semiconductor laser with a polygon filter [47].

The laser for the SS-OCT system was a high-speed wavelength-swept semiconductor laser with a polygon wavelength filter [47]. A unidirectional fibre optic ring, a semiconductor optical amplifier as the gain medium, and a novel polygonscanning filter were used. Centred at a $1310 \mathrm{~nm}$ wavelength with a bandwidth of $\sim 110$ $\mathrm{nm}$ and scan rate of $36 \mathrm{kHz}$, this laser was built with components that provided optimized power and tuning speed. Figure 3-2 has a schematic of this laser setup.

Raw OCT signals were continuously acquired by the ATS460 (AlazarTech) data acquisition card, and transferred to the memory of the video graphics card GeForce 9800 GT (NVIDIA). To manipulate and reconstruct the OCT images, custom-written kernels with the built-in CUDA libraries were utilized for massive parallel data processing. CUDA libraries and a specific DAQ card were used to enhance data throughput, while freeing up CPU resources. Direct data processing on the graphics card allowed the reconstructed OCT image to be displayed without the use of additional memory 
transactions, thus allowing real-time image acquisition, processing, and display to be only limited by the A-scan rate of the laser.

\subsection{Animal Experimental Setup}

The preparations for the animal experiment included the setup of the Animal Use Protocol (AUP 1280.8) at the University Health Network (Ontario Cancer Institute) at Princess Margaret Hospital in downtown Toronto, ordering the nude mice for the experiment, acquiring and growing the tumour cell line for tumour implantation, setting up and performing the dorsal skin window chamber surgeries, and acquiring the drug for chemotherapy treatment. Refer to Appendix A for an excerpt of the Animal Use Protocol used for this mice experiment.

\subsubsection{Animal Protocol Design}

A functional extension of optical coherence tomography capable of detecting cell death in vitro led to an initial $\mathrm{n}=3$ tumour mouse model study to test the feasibility of this imaging technique in vivo [12]. In this thesis, this same technique was performed in an $\mathrm{n}=10$ mice study to further evaluate the technique's effectiveness in cell death detection and obtain stronger statistical significance. Photodynamic therapy had been previously used to induce tumour cell kill [48], but due to its significant vascular damage, a human bladder carcinoma cell line in conjunction with a single dose of cisplatin was used in this feasibility test to evaluate the efficacy of this functional imaging technique.

The dorsal skin-fold window chamber, used previously in PDT and OCT microvasculature studies $[11,12,48]$, was mounted on ten female nude mice (NCRNU-F, 
Taconic), providing a suitable optical window for imaging and treatment of the microvasculature. The tumours were implanted into the fascia of the subcutaneous layer on the skin opposite of the window. A total volume of $10 \mu \mathrm{l}$ of HT-1376 (ATCC) human bladder carcinoma cells, suspended in growth medium, was injected at a concentration of $1.3 \times 10^{7}$ cells $/ \mathrm{mL}$. This cell line was grown at the Advanced Biomedical Ultrasound Imaging and Therapy Lab at Ryerson University. During this process, the mice were placed under ketamine/xylazine $(10 / 80 \mathrm{mg} / \mathrm{kg})$ anesthesia and kept warm on a heated pad. The mice were allowed to recover and were assessed on a daily basis during a twoweek period of tumour growth until approximately 1-2 $\mathrm{mm}$ in diameter.

Table 3-1: Mice experiment chart detailing the mice and the performed treatments and sacrifice date.

\begin{tabular}{|c|c|c|c|c|c|c|c|}
\hline Mice & $\begin{array}{c}\text { Window } \\
\text { Chamber }\end{array}$ & $\begin{array}{c}\text { Tumor } \\
\text { Implantation }\end{array}$ & $\begin{array}{c}\text { Cisplatin } \\
\text { Injection }\end{array}$ & $\begin{array}{c}\text { Day 0 } \\
\text { Imaging }\end{array}$ & $\begin{array}{c}\text { Day 1 } \\
\text { Imaging }\end{array}$ & $\begin{array}{c}\text { Day 2 } \\
\text { Imaging }\end{array}$ & Day to Sacrifice \\
\hline$\# 1$ & $\checkmark$ & $\checkmark$ & & $\checkmark$ & & & 0 \\
\hline$\# 2$ & $\checkmark$ & $\checkmark$ & & $\checkmark$ & $\checkmark$ & & 1 \\
\hline$\# 3$ & $\checkmark$ & $\checkmark$ & & $\checkmark$ & $\checkmark$ & $\checkmark$ & 2 \\
\hline$\# 4$ & $\checkmark$ & $\checkmark$ & $\checkmark$ & $\checkmark$ & $\checkmark$ & & 1 \\
\hline$\# 5$ & $\checkmark$ & $\checkmark$ & $\checkmark$ & $\checkmark$ & $\checkmark$ & & 1 \\
\hline$\# 6$ & $\checkmark$ & $\checkmark$ & $\checkmark$ & $\checkmark$ & $\checkmark$ & & 1 \\
\hline$\# 7$ & $\checkmark$ & $\checkmark$ & $\checkmark$ & $\checkmark$ & $\checkmark$ & $\checkmark$ & 2 \\
\hline$\# 8$ & $\checkmark$ & $\checkmark$ & $\checkmark$ & $\checkmark$ & $\checkmark$ & $\checkmark$ & 2 \\
\hline$\# 9$ & $\checkmark$ & $\checkmark$ & $\checkmark$ & $\checkmark$ & $\checkmark$ & $\checkmark$ & 2 \\
\hline$\# 10$ & $\checkmark$ & $\checkmark$ & $\checkmark$ & $\checkmark$ & $\checkmark$ & $\checkmark$ & 2 \\
\hline
\end{tabular}

Table 3-1 depicts the treatments performed and sacrifice date of each of the ten mice in this in vivo tumour study. All ten mice were treated with a $100 \mathrm{mg} / \mathrm{m}^{2}$ dose of chemotherapy drug cisplatin via tail vein injection after DLS-OCT imaging on Day 0. The imaging data was obtained again on Day 1 and Day 2 (time points 24 hours and 48 hours after cisplatin injection). In total, there were three control mice, three mice for the "Day 1" datapoint (24 hours after cisplatin exposure), and four mice for the "Day 2" 
datapoint (48 hours after cisplatin exposure). All procedures including surgeries, cisplatin injection, and imaging were conducted under ketamine-xylazine anesthesia $(10 / 80$ $\mathrm{kg} / \mathrm{mg}$ ) administered intraperitoneally.

The original treatment regimens of several mice in certain designated groups (as outlined in Table 3-1) were modified due to premature mouse deaths. It is important to outline these modifications so that the results from the thesis match with the original data sets taken during the experiment. An example of these survival changes was a mouse that was previously planned to be sacrifice on Day 2 (Mouse 3). Because it showed signs of weakened health, it was sacrificed on Day 0 instead. The initially planned mouse for Day 0 sacrifice (Mouse 1) was sacrificed on Day 1, and the initially planned mouse for Day 1 sacrifice (Mouse 2) was sacrificed on Day 2. The new mice experiment chart is shown in Table 3-2.

Table 3-2: Revised mice experiment chart detailing the mice and the performed treatments and sacrifice date.

\begin{tabular}{|c|c|c|c|c|c|c|c|}
\hline Mice & $\begin{array}{c}\text { Window } \\
\text { Chamber }\end{array}$ & $\begin{array}{c}\text { Tumor } \\
\text { Implantation }\end{array}$ & $\begin{array}{c}\text { Cisplatin } \\
\text { Injection }\end{array}$ & $\begin{array}{c}\text { Day 0 } \\
\text { Imaging }\end{array}$ & $\begin{array}{c}\text { Day 1 } \\
\text { Imaging }\end{array}$ & $\begin{array}{c}\text { Day 2 } \\
\text { Imaging }\end{array}$ & Day to Sacrifice \\
\hline$\# 3$ & $\checkmark$ & $\checkmark$ & & $\checkmark$ & & & 0 \\
\hline$\# 1$ & $\checkmark$ & $\checkmark$ & & $\checkmark$ & $\checkmark$ & & 1 \\
\hline$\# 2$ & $\checkmark$ & $\checkmark$ & & $\checkmark$ & $\checkmark$ & $\checkmark$ & 2 \\
\hline$\# 4$ & $\checkmark$ & $\checkmark$ & $\checkmark$ & $\checkmark$ & $\checkmark$ & & 1 \\
\hline$\# 7$ & $\checkmark$ & $\checkmark$ & $\checkmark$ & $\checkmark$ & $\checkmark$ & & 1 \\
\hline$\# 9$ & $\checkmark$ & $\checkmark$ & $\checkmark$ & $\checkmark$ & $\checkmark$ & & 1 \\
\hline$\# 5$ & $\checkmark$ & $\checkmark$ & $\checkmark$ & $\checkmark$ & $\checkmark$ & $\checkmark$ & 2 \\
\hline$\# 6$ & $\checkmark$ & $\checkmark$ & $\checkmark$ & $\checkmark$ & $\checkmark$ & $\checkmark$ & 2 \\
\hline$\# 8$ & $\checkmark$ & $\checkmark$ & $\checkmark$ & $\checkmark$ & $\checkmark$ & $\checkmark$ & 2 \\
\hline$\# 10$ & $\checkmark$ & $\checkmark$ & $\checkmark$ & $\checkmark$ & $\checkmark$ & $\checkmark$ & 2 \\
\hline
\end{tabular}

\subsection{Data Acquisition and Analysis}


For each mouse imaged, a total of twenty-six imaging planes of two-dimensional frames of OCT data were acquired continuously for 1000 frames, and spanned over the whole tumour area of the window chamber. The time required for imaging the 1000 frames was 10 seconds. Therefore, the frame rate for imaging the two-dimensional planes was 100 frames per second. The imaging planes were spaced approximately $25 \mu \mathrm{m}$ apart and covered the tumour and non-tumour skin regions. The frame acquisition rate was 200 frames per second. For each frame, $360 \mathrm{~A}$-scans covered the entire $3 \mathrm{~mm}$ lateral distance. Figure 3-3 has a schematic of this experimental setup. Refer to Appendix B for more images of the experimental setup.

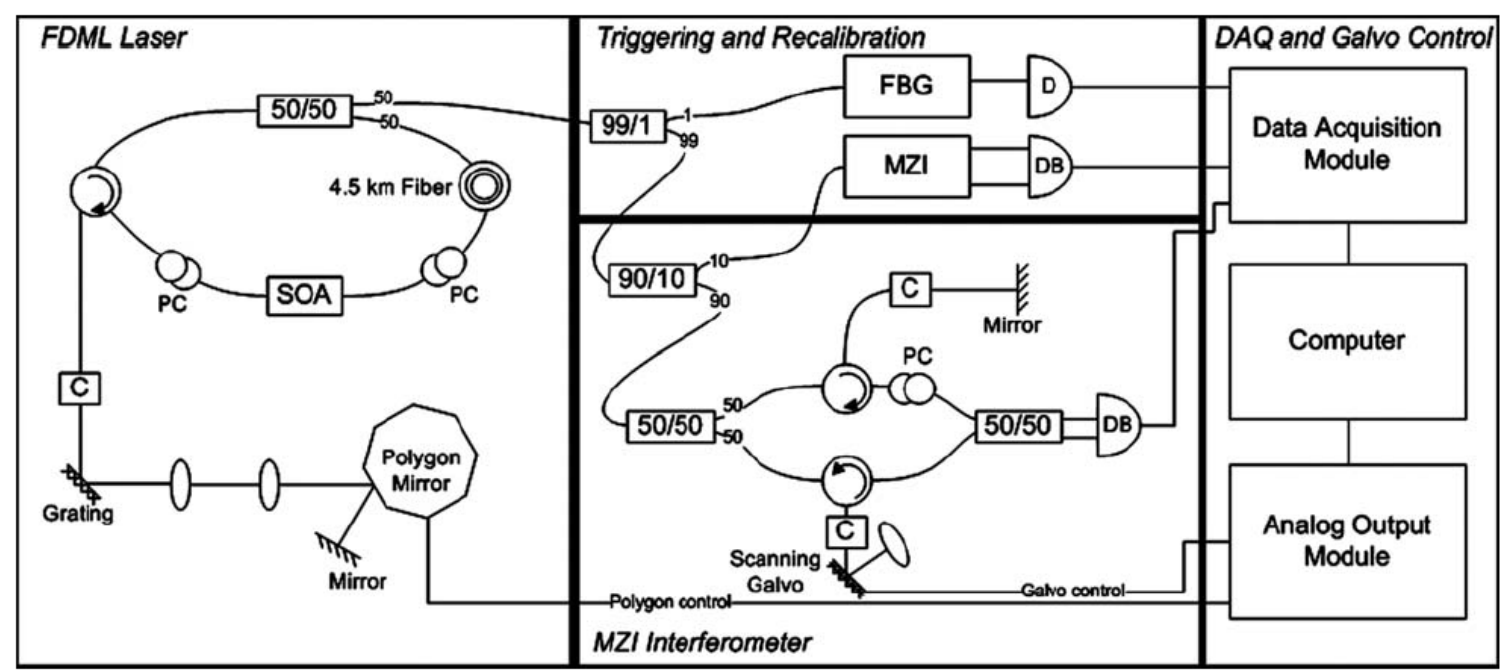

Figure 3-3: Schematic for the SS-OCT system. SOA, semiconductor optical amplifier; PC, polarization controller; C, collimator; FBG, fibre Bragg grating; MZI, Mach-Zehnder interferometer; D, detector; DB, dual balanced detector [48].

The multiple two-dimensional frames for each imaging plane were used to generate decorrelation time (DT) maps. The methods established by Farhat et al. were used for the analysis of the OCT data [12]. The DT for each pixel of each imaging plane was determined by calculating the autocorrelation function of the fluctuating signal by 
taking the inverse Fourier transform of the power spectrum [11]. The autocorrelation function and the signal's power spectrum are Fourier transform pairs. Vital to the calculation of the autocorrelation function was the static scattering component of the tissue. The tumour tissue samples were assumed to be a nonergodic medium, thus required the removal of the static scattering component from the fluctuating signal. This was done by the "heterodyne approach" [45] that removed the static scattering contribution of the 7 by 7 pixel region centred at the specific pixel from the intensity autocorrelation function to produce the heterodyne intermediate scattering function (ISF). The 1/e value of the heterodyne ISF was then used to calculate the DT that was displayed in the final DT map in conjunction with 3 by 3 spatial averaging. Refer to Appendix C for the MATLAB code that was used to generate the DT maps, and Appendix D for further explanation on the equations used in the "heterodyne approach".

The averages of the DT values within a selected region of interest were analyzed to observe tumour changes due to intracellular and extracellular motion. Thresholding was applied to the DT maps to remove the contribution from rapid bulk motion and blood flow (the results from the thresholding are presented in Section 4.2). The DT values that were less than the threshold value were reassigned to a value of zero. A modified "jet" colour bar setting in MATLAB was used for the post-threshold DT maps, with black coloured pixels indicating an assigned DT value of zero. Statistical analysis via the Student's t-test was performed to compare the means of the average DT values of the control and cisplatin mice.

\subsection{Histology}


The ten mice in this in vivo tumour study were sacrificed according to Table 3-2. For each mouse, the tumour was excised and fixed in 10\% buffered formalin for 24 to 48 hours before submission to the University Health Network Pathology Research Program. The tissue samples were processed for hematoxylin and eosin (H\&E) staining. The subsequent slides were scanned using a Leica DC 200 digital imaging system (Leica Microsystems $\mathrm{GmbH}$, Germany) at 4x and 10x objective lens magnification. 


\section{Chapter 4: Results}

The results include the collection of two types of OCT images (OCT b-mode images and decorrelation time maps) from the dynamic light scattering optical coherence tomography (DLS-OCT) experiment and the corresponding tissue histology. OCT bmode images provide information on the structure and optical scattering properties of the sample. Decorrelation time maps provide information on the motion in sample tumour tissue. The histology images from hematoxylin and eosin (H\&E) staining provide a means for visualizing the different cellular structures of the sample.

\subsection{Images Acquired}

To perform DLS-OCT imaging, the swept source OCT imaging system was used to obtain the data that formed the b-mode images. Post-processing techniques were used to obtain the decorrelation time maps from the b-mode images using MATLAB software as described in Section 3.3. The histology images were prepared as described in Section 3.4 .

Representative images from the Mouse 6 and Mouse 10 tumours are presented in this chapter (even though all the data were collected according to Table 3-2). Mouse 6 and 10 were chosen primarily because: 1) both of these mice had data for the full threeday duration of the experiment enabling tumour analysis over time; and 2) both mice had images (b-mode, DT, and histology) that had full grown tumours of 1-2 mm in diameter.

\subsubsection{OCT B-mode images}




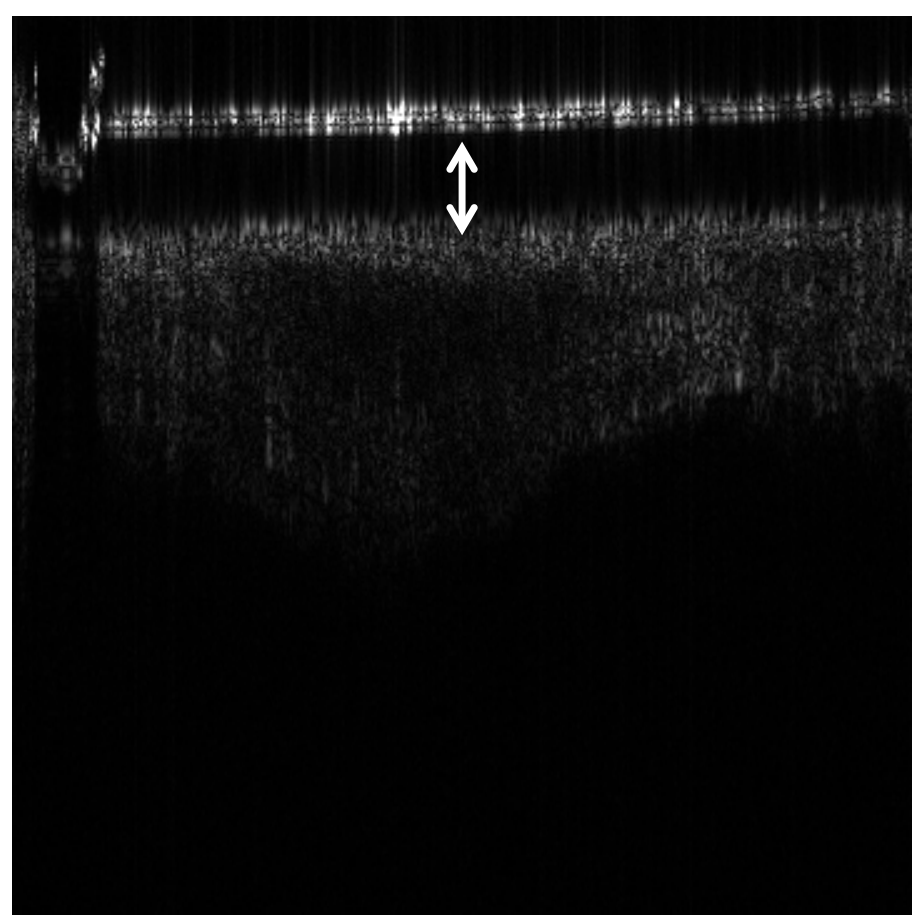

(a)

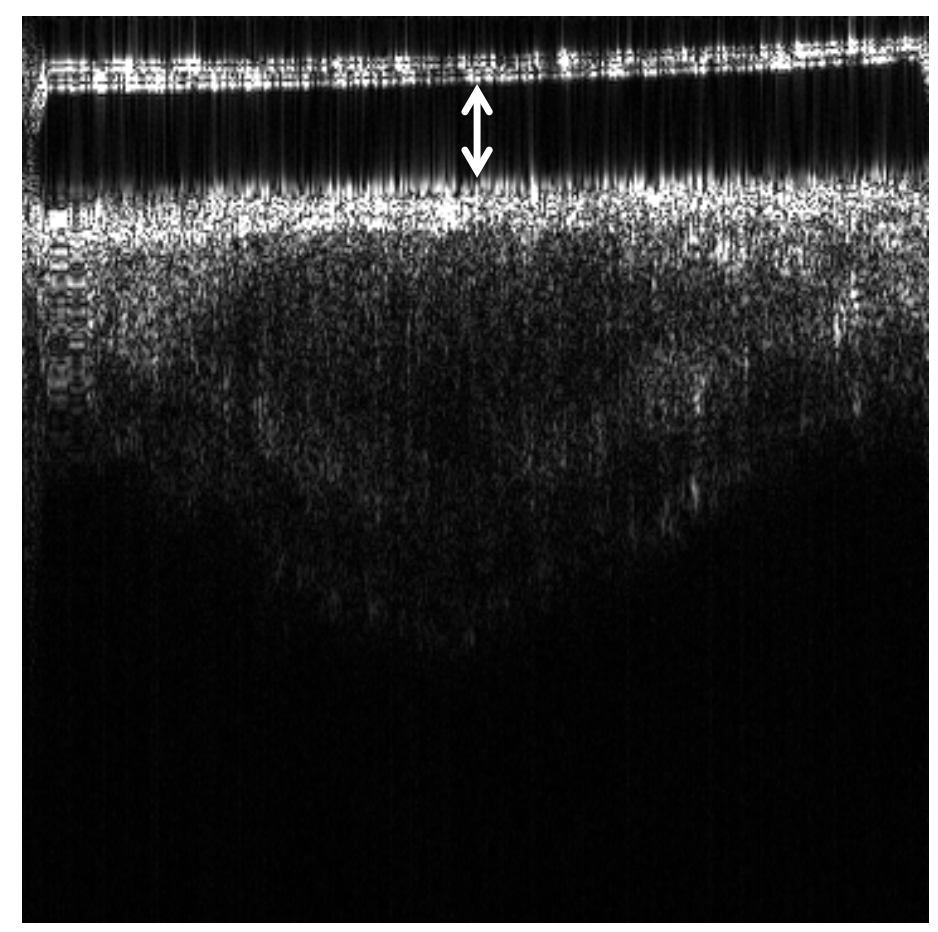

(b)

Figure 4-1: A cross-sectional (b-mode) image of the tumour for (a) Mouse 6 on Day 1 and (b) Mouse 10 on Day 2. The white arrows represent the $0.25 \mathrm{~mm}$ thickness of the glass coverslip. The image height and image width are both $3 \mathrm{~mm}$. 
Figures 4-1a and 4-1b are examples of cross-sectional (b-mode) OCT images taken at the location of a tumour implanted onto the dorsal skin of Mouse 6 and 10, respectively. These b-mode images were formed from depth profiles (A-scans) taken across a $3 \mathrm{~mm}$ lateral distance. The brightness of the pixels within these images indicates the intensity of the backscattered light received by the OCT detector. The b-mode images provide structural information of the sample based on the backscattering. In this example, the glass coverslip for the window chamber is defined by the dark area between the bright lines that lies above the highly scattering tumour sample. The tumour is confined to the area of reduced scattering in the image. A collection of b-mode images were subsequently used to formulate the respective decorrelation time map using postprocessing techniques.

\subsubsection{Decorrelation Time Maps}

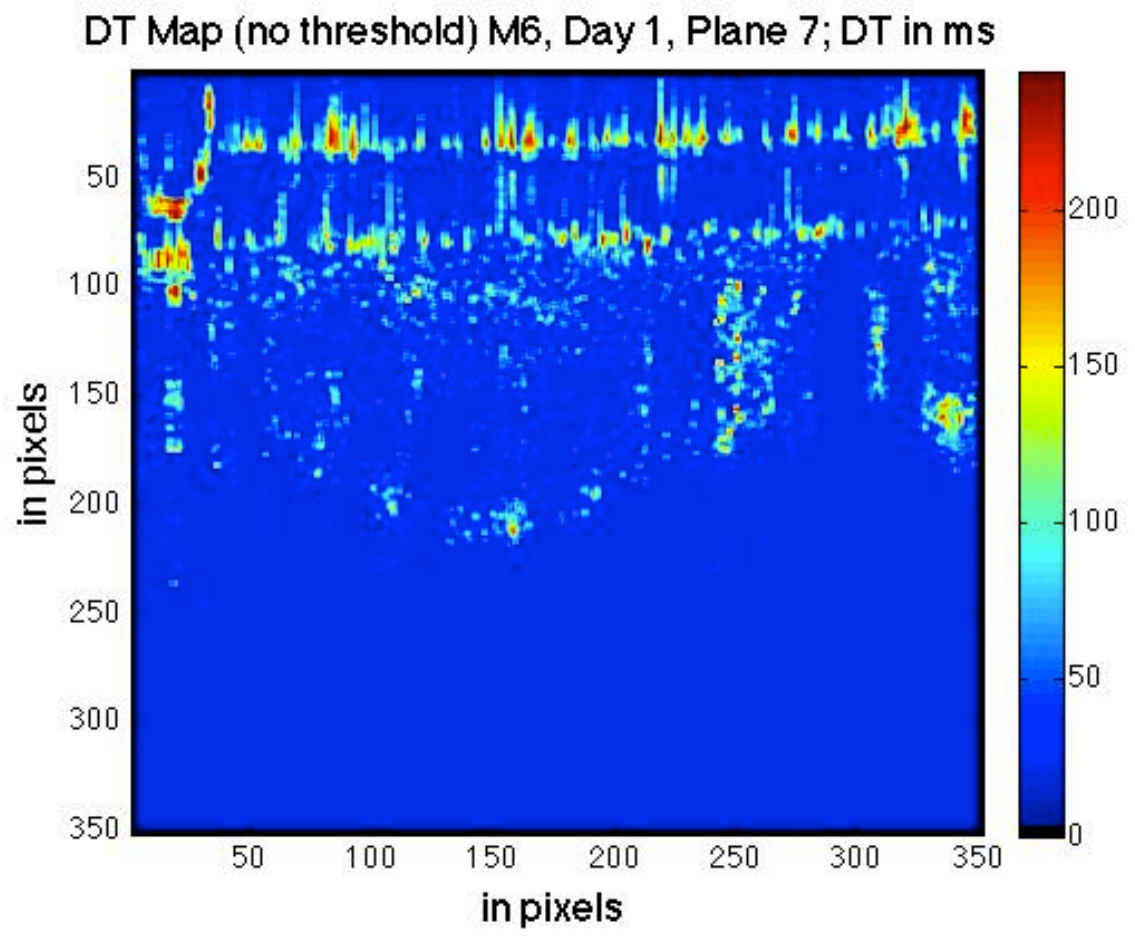

(a) 


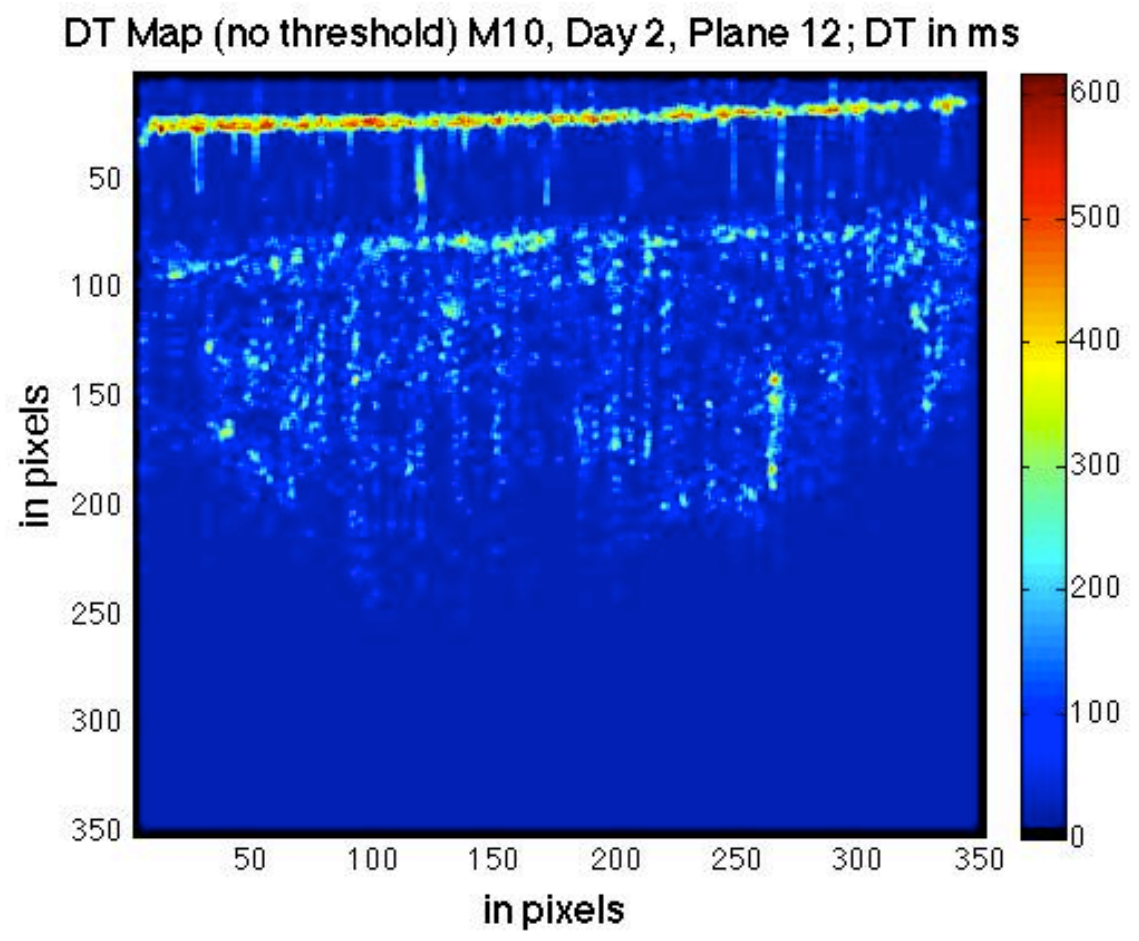

(b)

Figure 4-2: DT map of the mouse tumour produced from OCT b-mode images using a decorrelation-based algorithm for (a) Mouse 6 (Day 1) and (b) Mouse 10 (Day 2). The colour map represents the DT value in ms. The image height and image width are both 3 mm.

Figures $4-2 \mathrm{a}$ and $4-2 \mathrm{~b}$ are examples of decorrelation time (DT) maps produced from the original b-mode images using a decorrelation-based algorithm (described in Section 3.3) for Mouse 6 and 10, respectively. The initial data collected for each crosssectional imaging plane of the tumour included multiple frames (separated in time by 10 ms) imaged at 100 frames per second. Intensities at a specific pixel location across the acquired frames were calculated by the algorithm. An example of the fluctuations in signal intensity is shown in Figure 4-3a for Mouse 6 (Figure 4-4a for Mouse 10) for frame 101 to 200 of the total 1000 frames of data imaged. The power spectrum of the pixel intensities is shown in Figure 4-3b (Figure 4-4b for Mouse 10). The resulting 
inverse Fourier transform of the power spectrum of the signal intensities of the same pixel is shown in Figure 4-3c (Figure 4-4c for Mouse 10). The intermediate scattering function (ISF, from the "heterodyne approach") of the same pixel is shown in Figure 4-3d (Figure 4-4d for Mouse 10). Lastly, the curve showing the DT value recorded in the DT map (determined from the heterodyne ISF for each pixel as the time it takes for the signal to fall below the value of 1/e) is displayed in Figure 4-3e (Figure 4-4e for Mouse 10). For Figure 4-3, point $(128,154)$ of plane 7 for Mouse 6 on Day 1 was chosen as a pixel with a DT of 86.67 ms. For Figure 4-4, point $(150,150)$ of plane 12 for Mouse 10 on Day 2 was chosen as a pixel with a DT of $20 \mathrm{~ms}$.

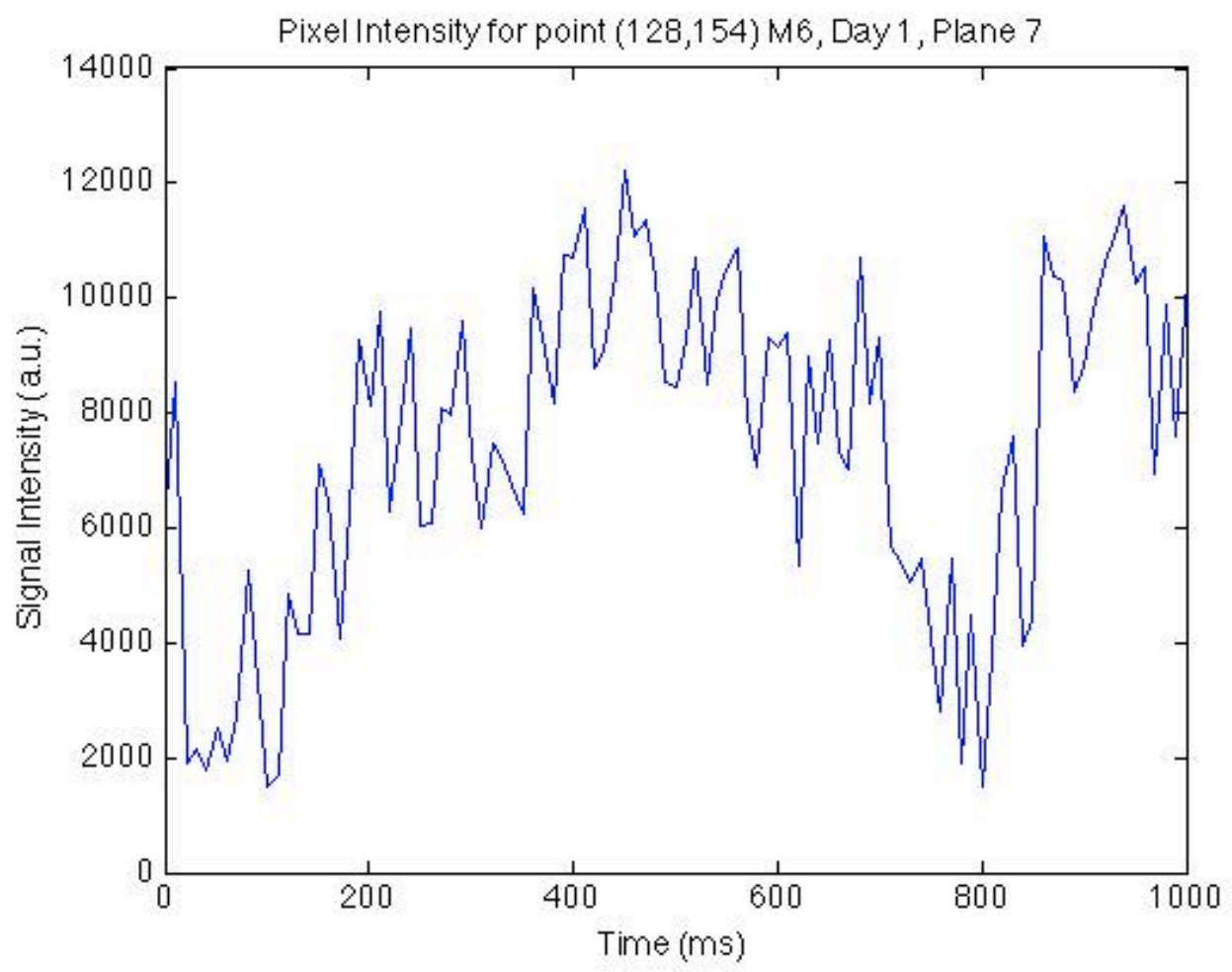

(a) 


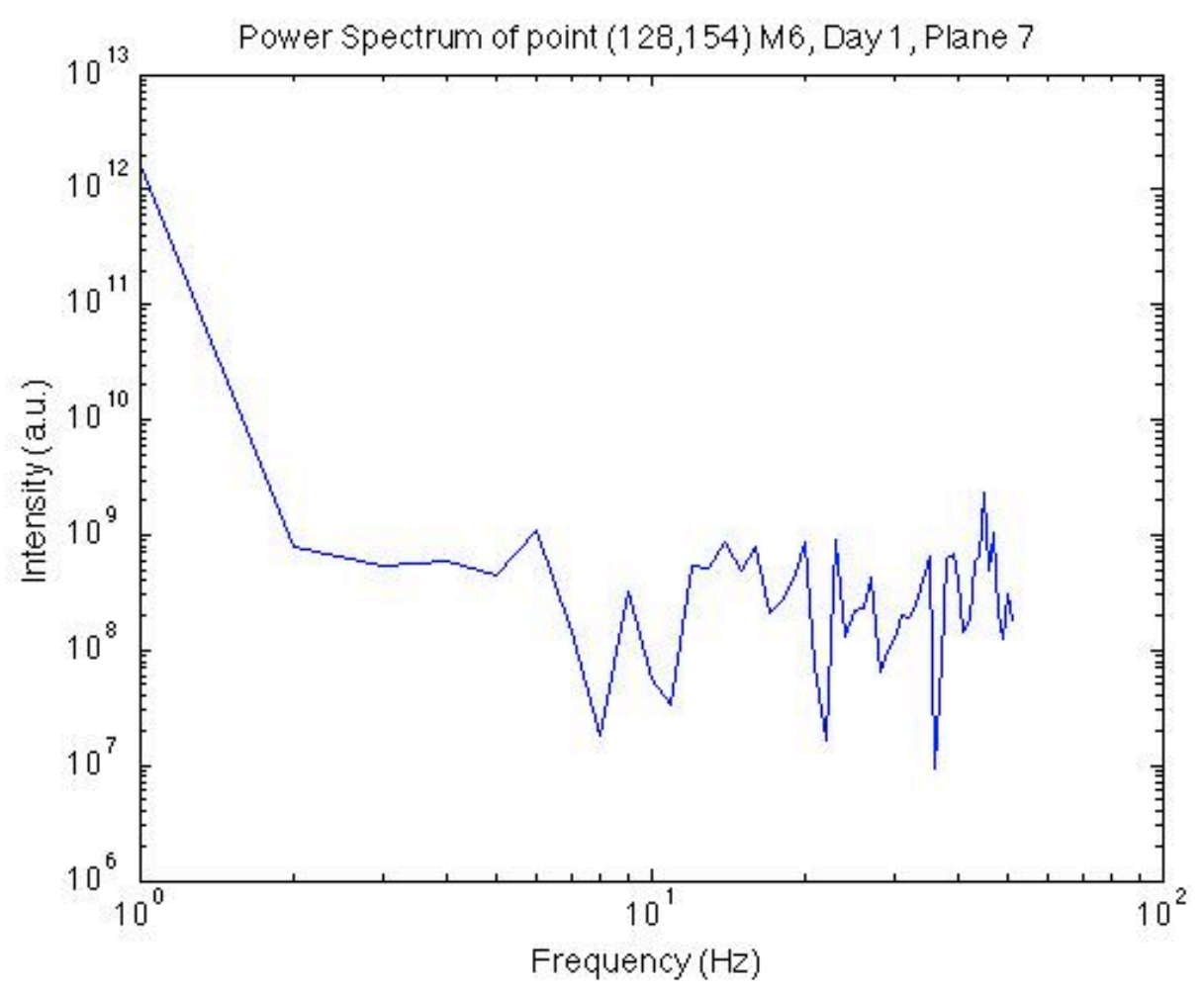

(b)

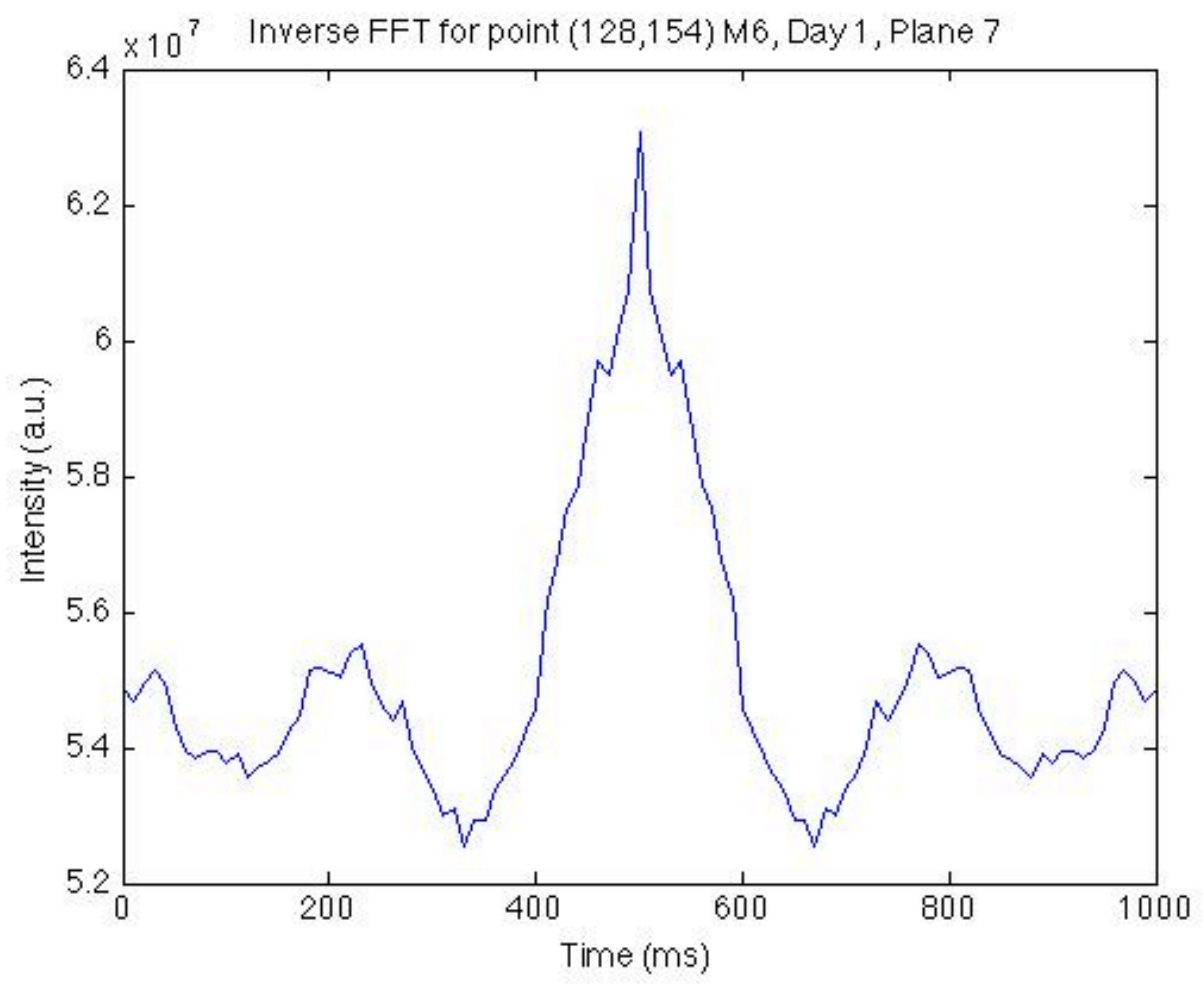

(c) 


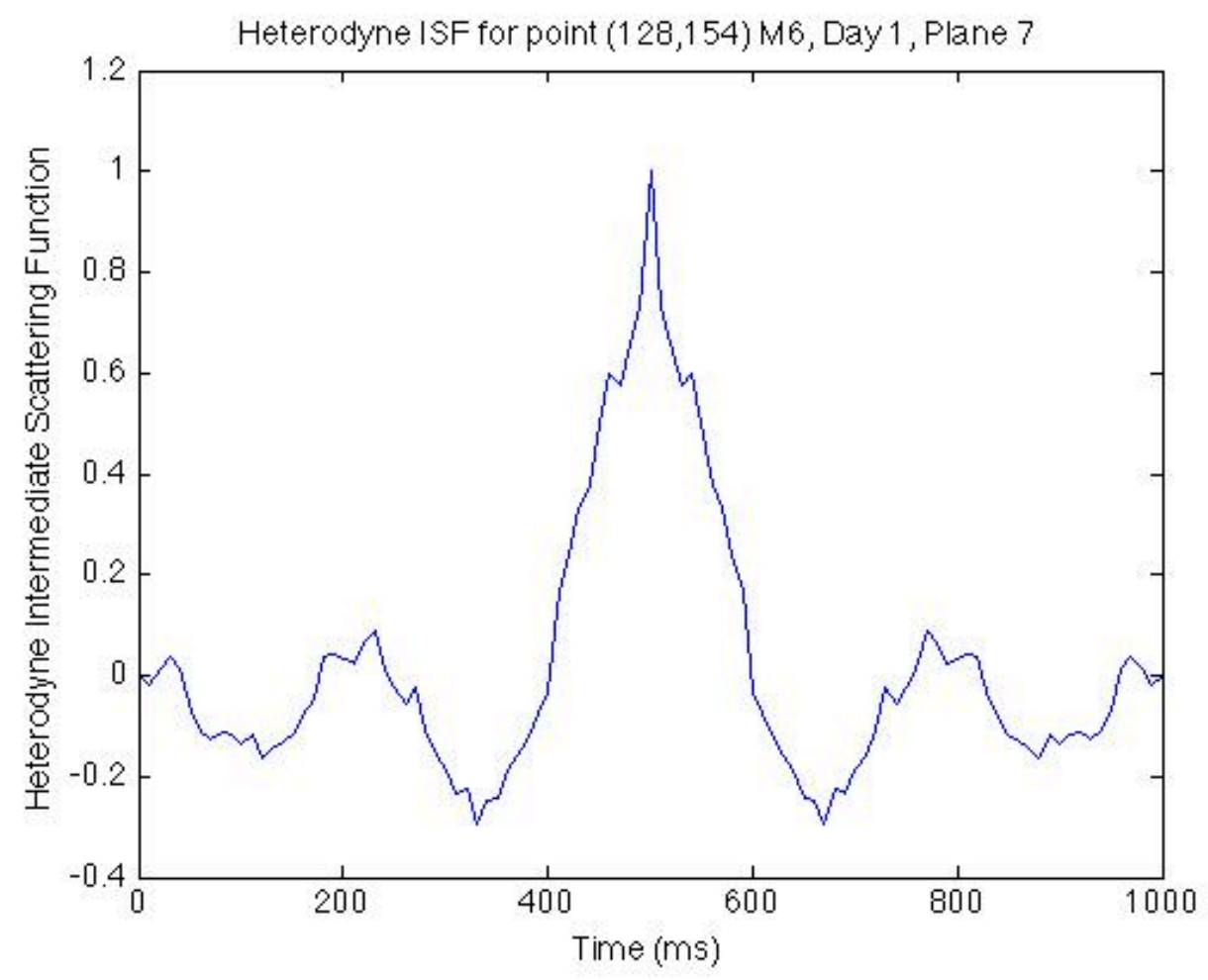

(d)

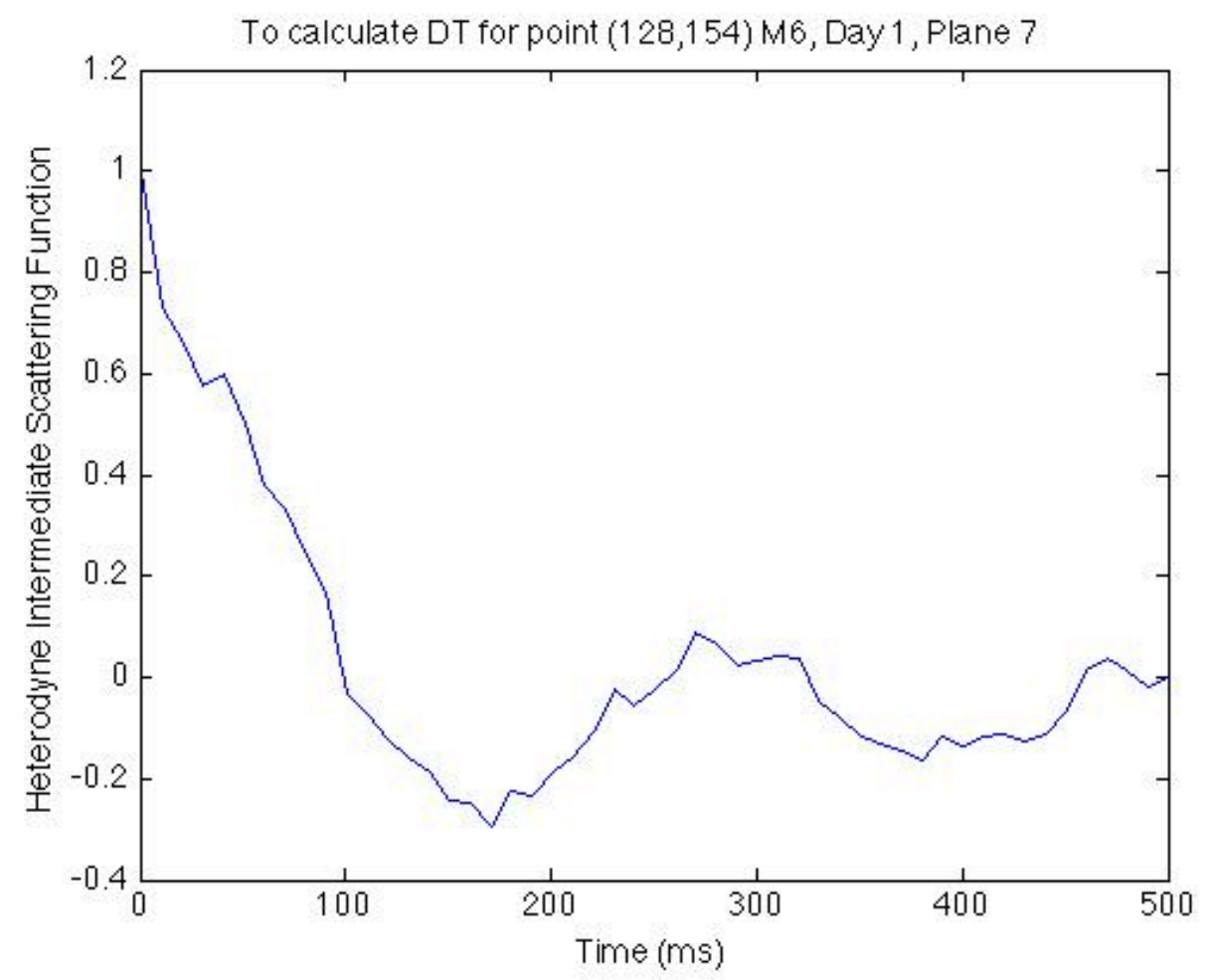

(e) 
Figure 4-3: (a) The fluctuations in signal intensity for point $(128,154)$ across multiple OCT timeframes for Mouse 6 (Day 1); (b) The power spectrum of the pixel intensities in (a); (c) The inverse Fourier transform of the power spectrum in (b); (d) The heterodyne intermediate scattering function produced using the Joosten algorithm [45]; (e) The resulting curve used to choose the DT value (the decorrelation time is the time chosen when the heterodyne ISF drops below the value of $1 / \mathrm{e}$ ).

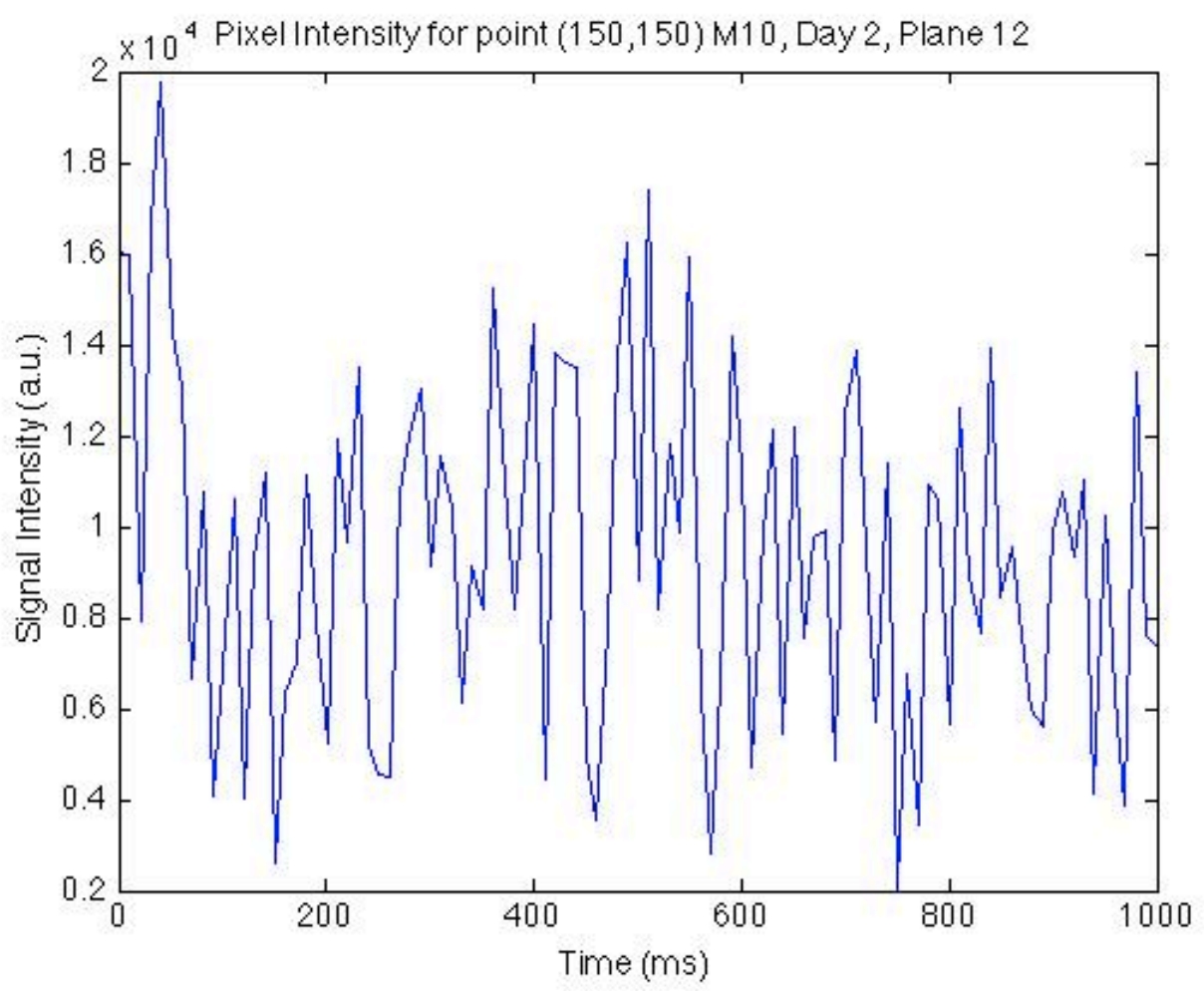

(a) 


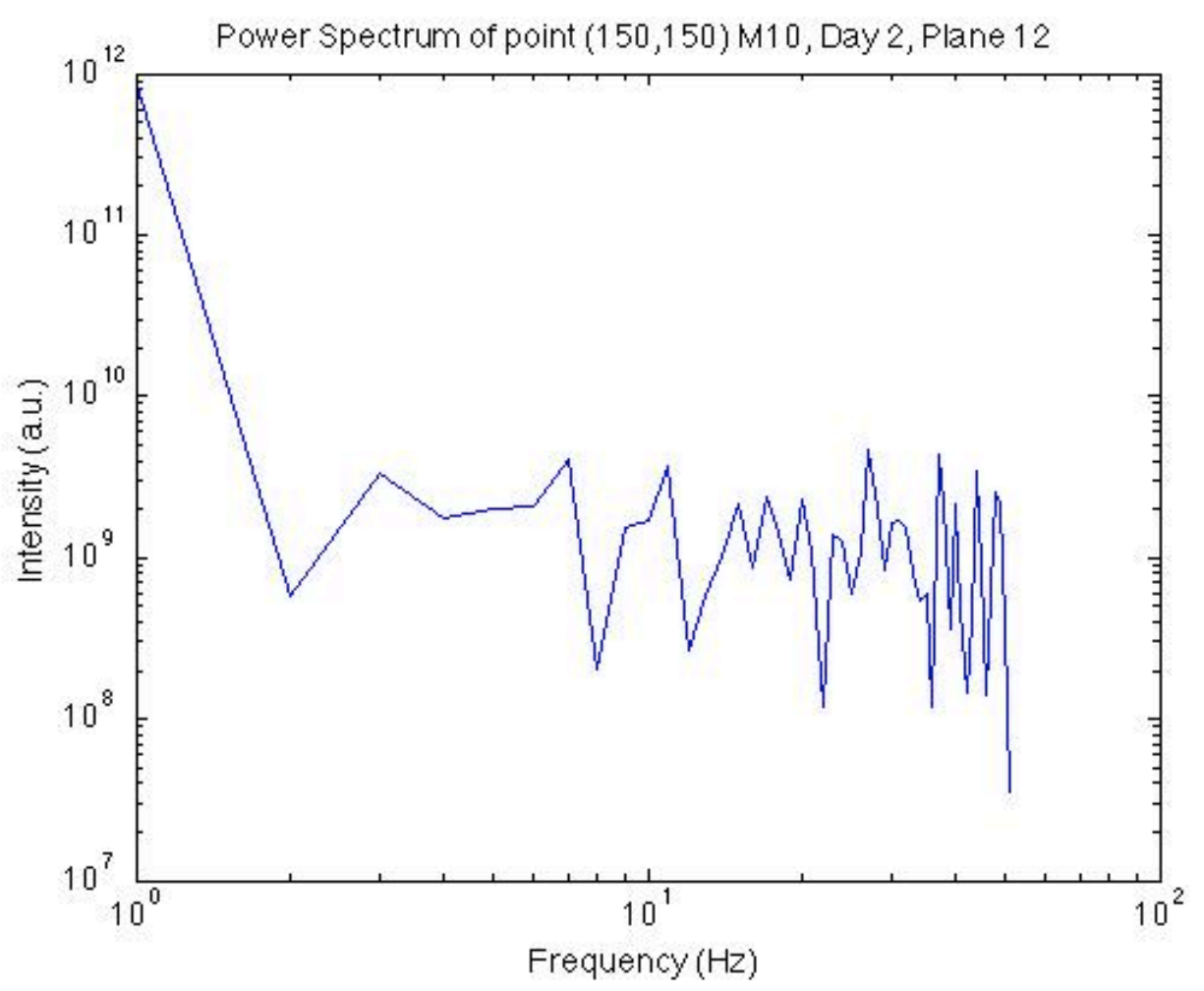

(b)

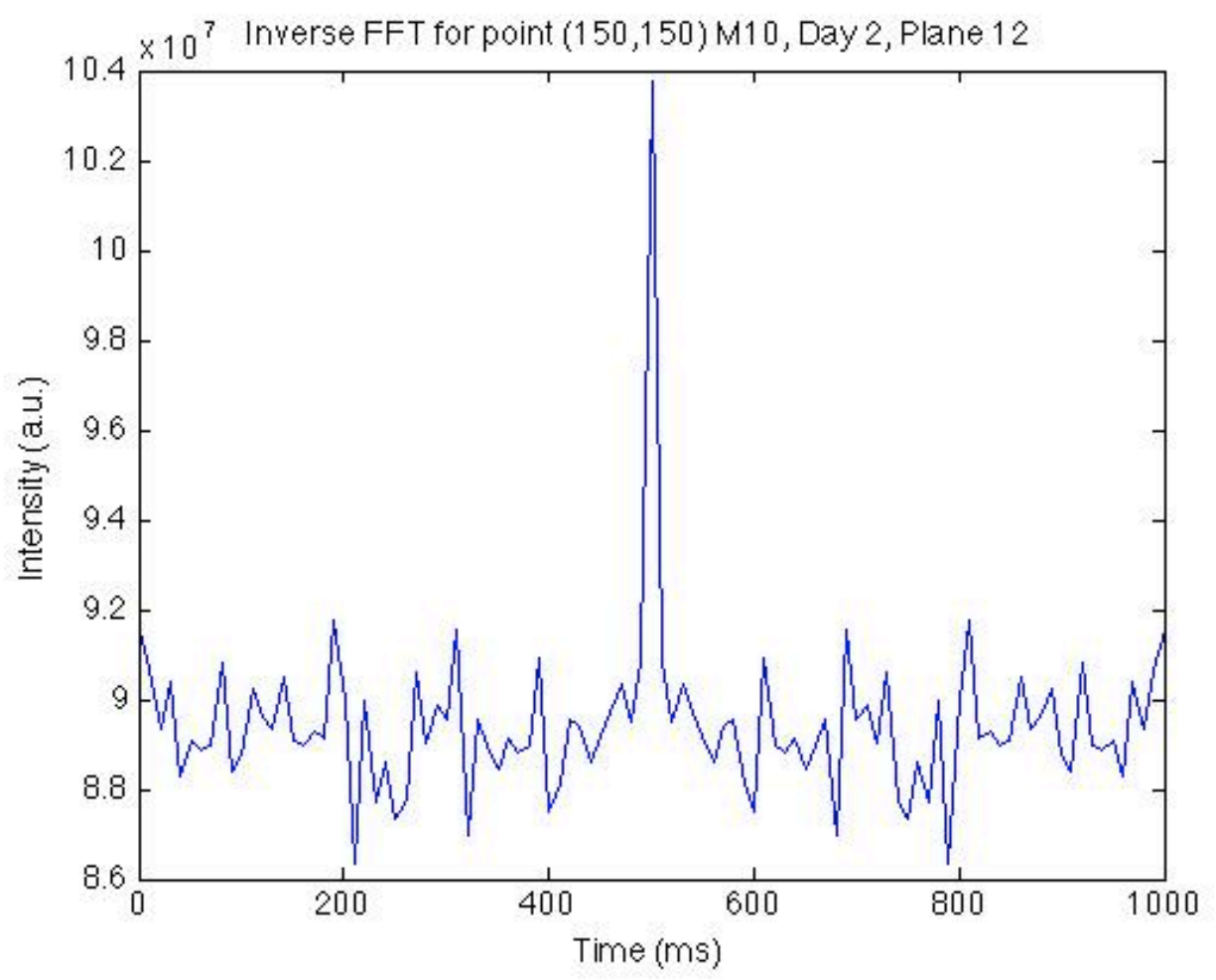

(c) 


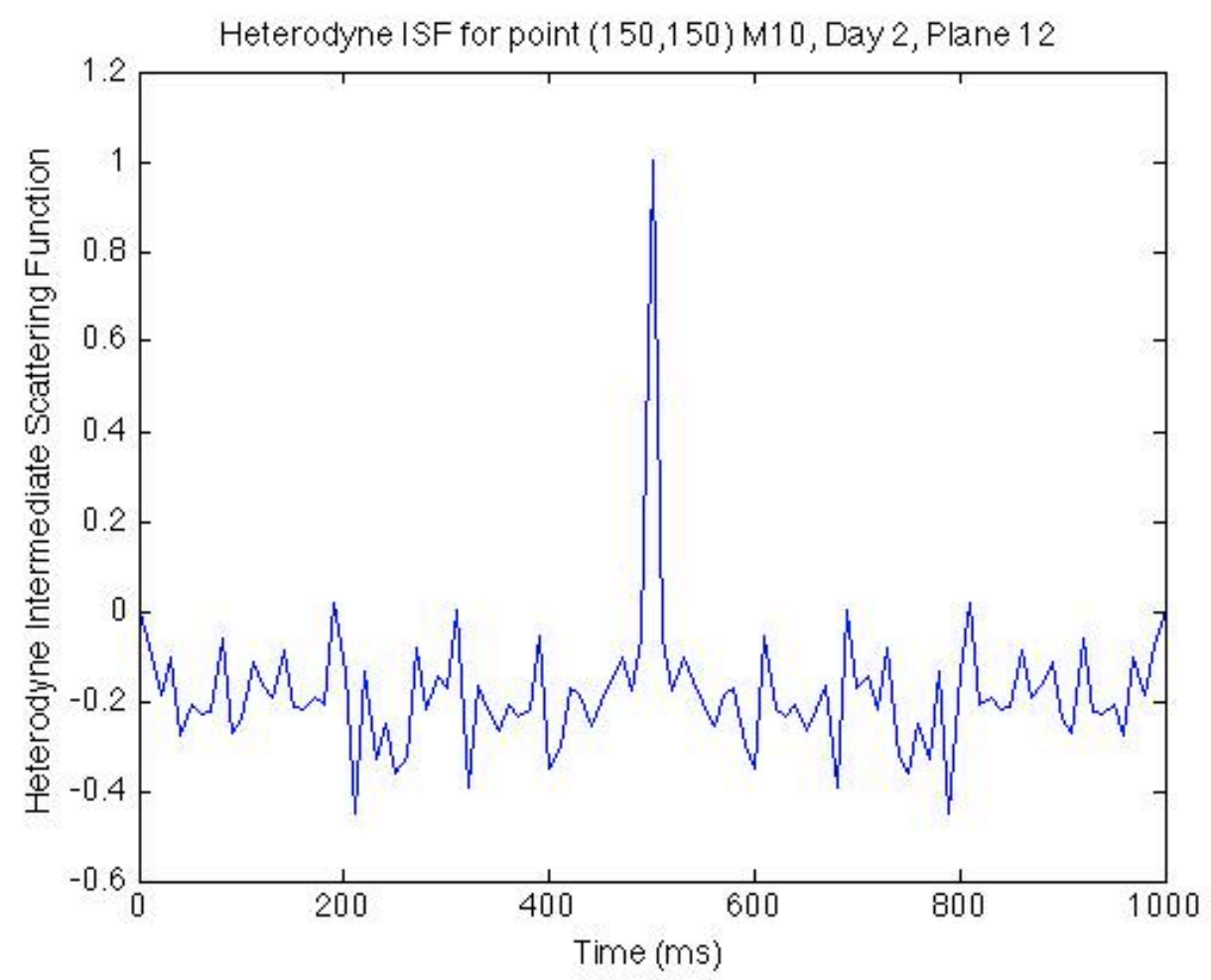

(d)

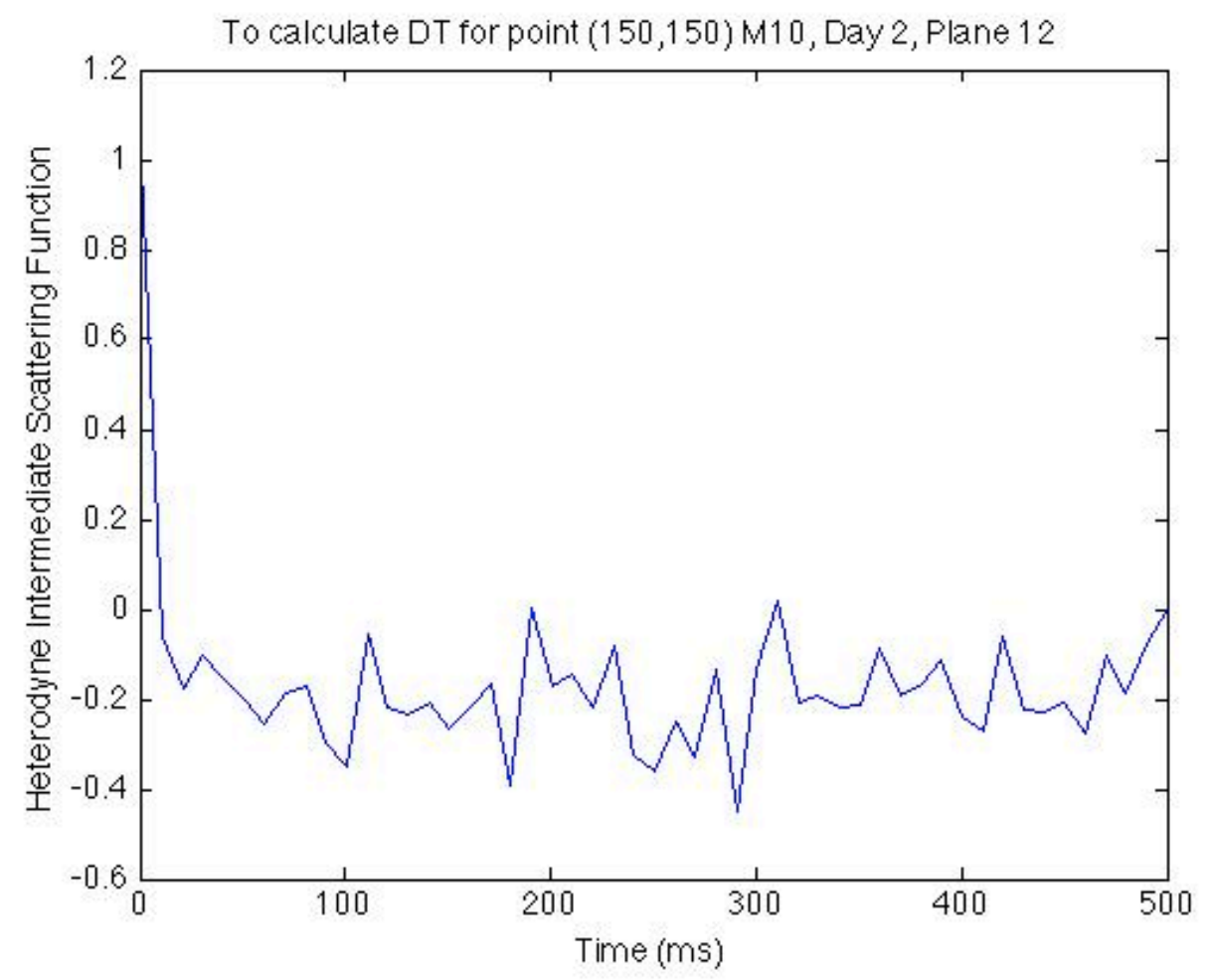

(e) 
Figure 4-4: (a) The fluctuations in signal intensity for point $(150,150)$ across multiple OCT timeframes for Mouse 10; (b) The power spectrum of the pixel intensities in (a); (c)

The inverse Fourier transform of the power spectrum in (b); (d) The heterodyne intermediate scattering function produced using the Joosten algorithm [45]; (e) The resulting curve used to choose the DT value (the decorrelation time is the time chosen when the heterodyne ISF drops below the value of 1/e).

Figure 4-3 and 4-4 show the fluctuations in signal intensity for a pixel corresponding to frame 101 to 200 of the specific imaging plane. Only 100 frames were chosen to perform the decorrelation algorithm quickly. The same algorithm can be repeated for other sections of the 1000 frames to compare the DT of the same pixel over time. Table 4-1 shows the DT measured for Mouse 2 (control) and Mouse 6 (treated) on Day 1 for 10 sections of the 1000 frames of data. Two pixels were chosen, where one was at a location suspected for longer DT and the other at a location suspected for shorter DT.

Table 4-1: Decorrelation times taken at a long DT pixel and short DT pixel for Mouse 2 and Mouse 6 over ten 100-frame sections.

\begin{tabular}{ccc|ccc}
\hline \multicolumn{3}{c|}{ Mouse 2 (control) } & \multicolumn{3}{c}{ Mouse 6 (cisplatin treated) } \\
\hline Frames & $\begin{array}{c}\text { Long DT at } \\
\text { Point (143, }\end{array}$ & $\begin{array}{c}\text { Short DT at } \\
\text { Point (143, }\end{array}$ & Frames & $\begin{array}{c}\text { Long DT at } \\
\text { Point (128, }\end{array}$ & $\begin{array}{c}\text { Short DT at } \\
\text { Point (150, }\end{array}$ \\
& 240) in ms & 230) in ms & & 154) in ms & 150) in ms \\
$\mathbf{1 - 1 0 0}$ & 177.8 & 20 & $\mathbf{1 - 1 0 0}$ & 60 & 20 \\
$\mathbf{1 0 1 - 2 0 0}$ & 175.6 & 20 & $\mathbf{1 0 1 - 2 0 0}$ & 86.67 & 20 \\
$\mathbf{2 0 1 - 3 0 0}$ & 166.7 & 20 & $\mathbf{2 0 1 - 3 0 0}$ & 66.67 & 20 \\
$\mathbf{3 0 1 - 4 0 0}$ & 155.6 & 20 & $\mathbf{3 0 1 - 4 0 0}$ & 51.11 & 20 \\
$\mathbf{4 0 1 - 5 0 0}$ & 128.9 & 20 & $\mathbf{4 0 1 - 5 0 0}$ & 64.44 & 20 \\
$\mathbf{5 0 1 - 6 0 0}$ & 140 & 20 & $\mathbf{5 0 1 - 6 0 0}$ & 26.67 & 20 \\
$\mathbf{6 0 1 - 7 0 0}$ & 91.11 & 20 & $\mathbf{6 0 1 - 7 0 0}$ & 60 & 20 \\
$\mathbf{7 0 1 - 8 0 0}$ & 140 & 20 & $\mathbf{7 0 1 - 8 0 0}$ & 64.44 & 20 \\
$\mathbf{8 0 1 - 9 0 0}$ & 135.6 & 20 & $\mathbf{8 0 1 - 9 0 0}$ & 24.44 & 20 \\
$\mathbf{9 0 1 - 1 0 0 0}$ & 148.9 & 22.22 & $\mathbf{9 0 1 - 1 0 0 0}$ & 53.33 & 20 \\
Mean & 146.0 & 20.2 & Mean & 55.78 & 20 \\
Std. Dev. & 25.61 & 0.70 & Std. Dev. & 18.61 & 0 \\
\hline
\end{tabular}

The data in Table 4-1 shows that the DT at a pixel location indicative of long or short DT remains long or short throughout the whole 1000 frames. There are slight changes for 
pixels with longer DT, but pixels with shorter DT remained the same for most of the pixels. This short DT of $20 \mathrm{~ms}$ was at the lowest possible DT value measured with the system. This pattern was observed for other pixel locations in all the mice in this study.

\subsubsection{Histology Images}

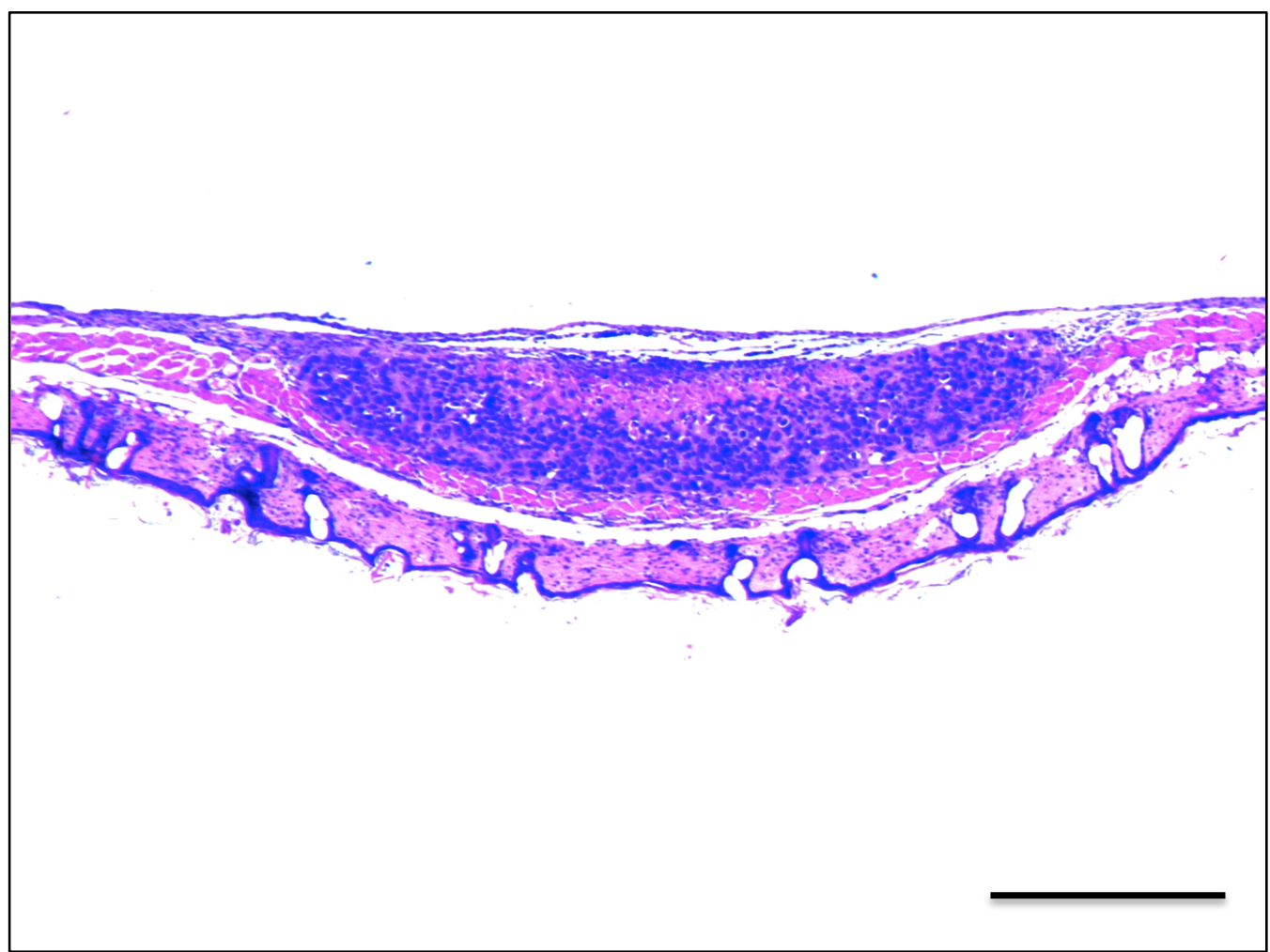

(a) 


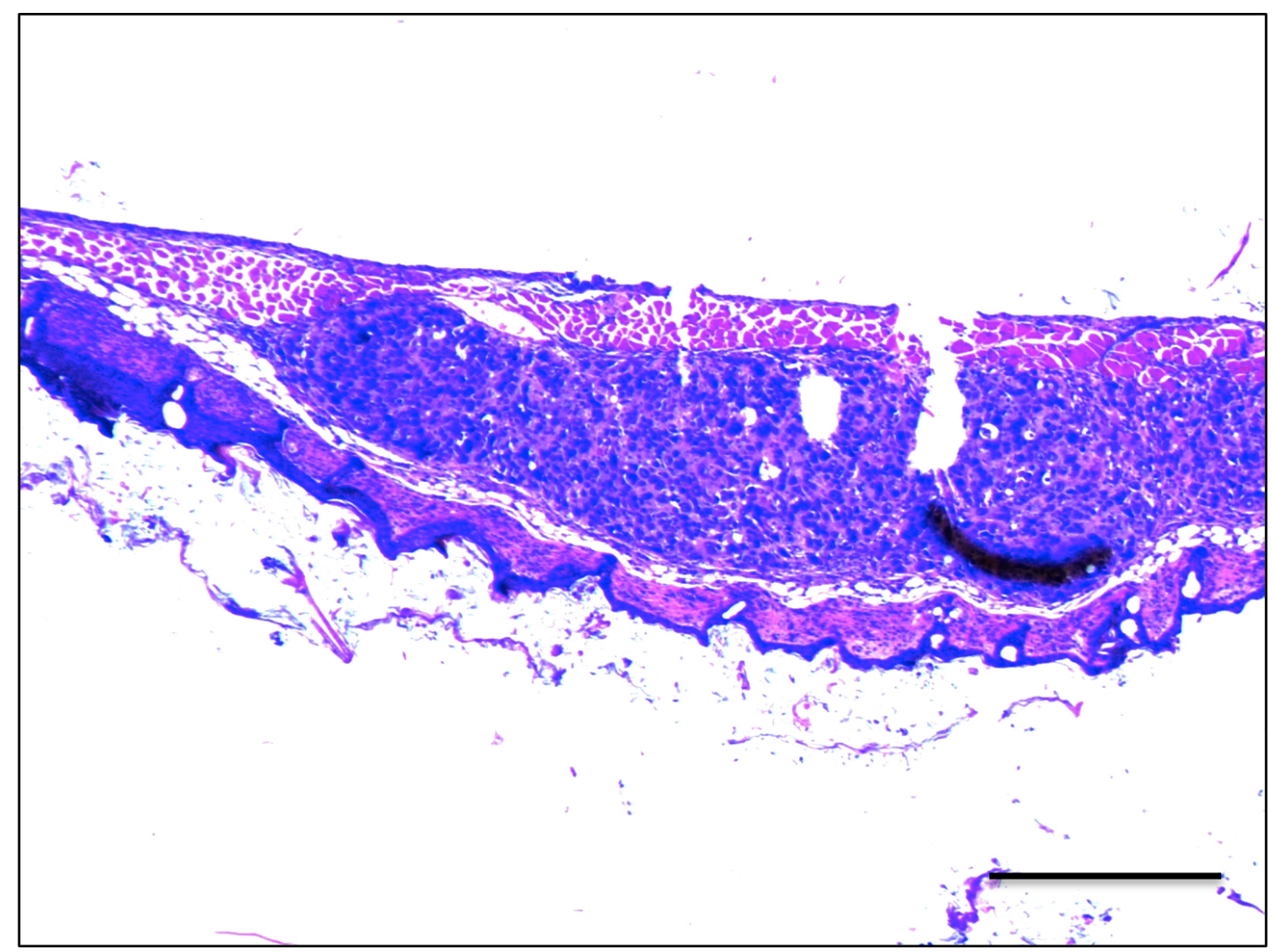

(b)

Figure 4-5: An example hematoxylin and eosin staining of the tumour imaged with $4 x$ microscope objective for (a) Mouse 6 and (b) Mouse 10. The scale bar is $500 \mu \mathrm{m}$.

Figures 4-5a and 4-5b show examples of H\&E stained images of a tumour tissue sample for Mouse 6 and 10, respectively. The tumour cells were implanted into the fascia of the subcutaneous tissue on the inside of the mouse dorsal skin. The glass window for the window chamber was placed on top of the subcutaneous tissue, thus resulting in a flat top surface for the tumour tissue sample. The H\&E staining showed that the tissue sample contained tumour cells apart from the skin cell morphology. Further histology analysis is described later in this chapter.

\subsection{Flow Segmentation}


The previous in vivo feasibility study by Farhat et al. included observations of areas with short DT, with the recognition that these areas are possible blood vessel locations [12]. Since the motion due to cell death is of interest in this thesis, the pixels for the locations of blood vessels should not be taken into account in the analysis. The analysis presented in this thesis will segment the locations of vessels assuming that areas of short DT correspond to potential locations of blood vessels. In order to measure an average DT value of a specific region of interest (ROI) that includes tumour tissue only (and not blood vessels), it was necessary to segment out pixels containing blood vessels as indicated by short DT values and/or the presence of shadows. The term "average DT" refers to the average of all the DT values within a selected ROI. A thresholding method was applied to the DT map in order to remove what we postulate are vessel flow contributions in the average DT calculations.

To obtain a suitable threshold value for flow segmentation, the DT value of tumour cells must be known. Previous in vitro studies have shown that the DT value for cells in cell aggregates emulating tumours range from 100 to $300 \mathrm{~ms}$ (Figure 2-7) [11]. In addition, speckle variance uses techniques similar to the ones used in this study to produce images of the vasculature and it is known that blood vessels cast a shadow in speckle variance measurements of the vasculature. This shadowing effect has been attributed to multiple scattering effects [49]. Therefore, there is strong evidence that short DT regions correspond to motion due to the presence of the flowing blood in blood vessels. 


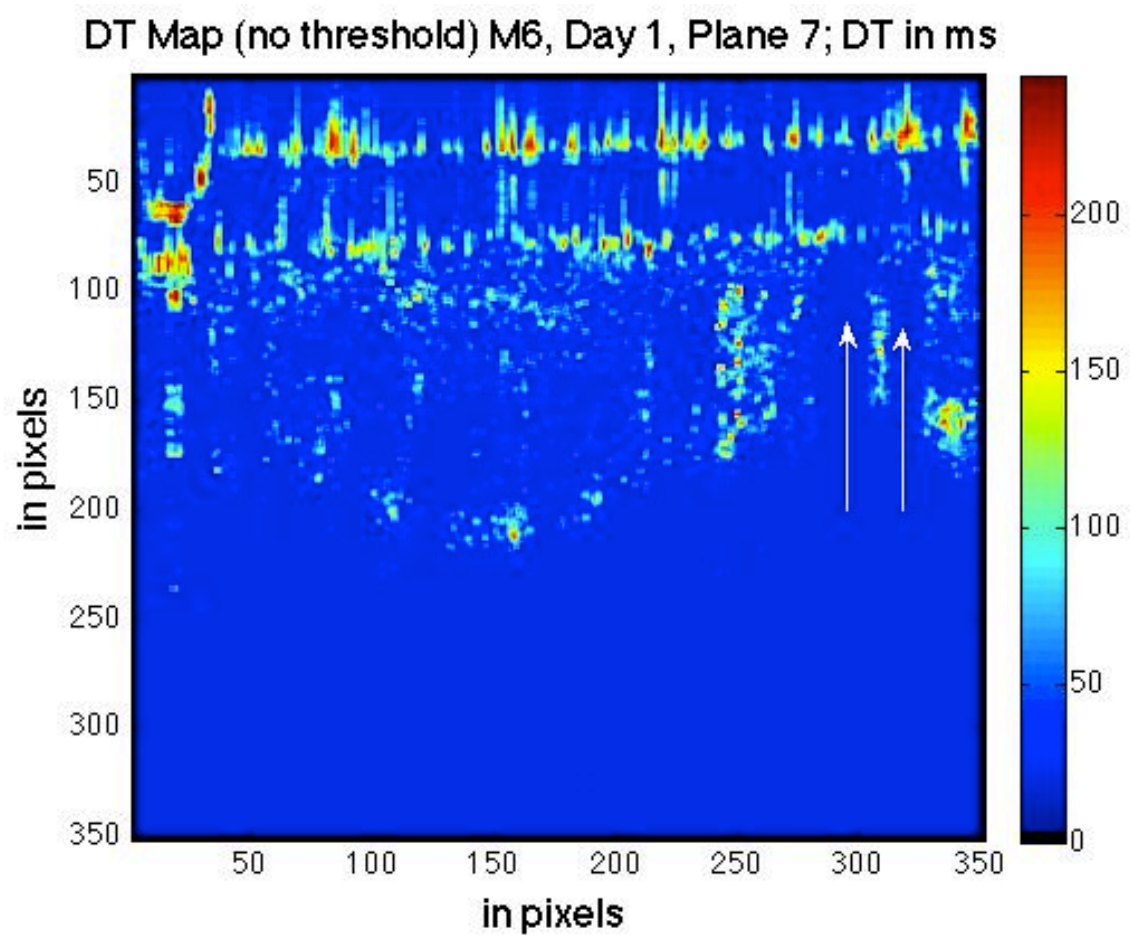

(a)

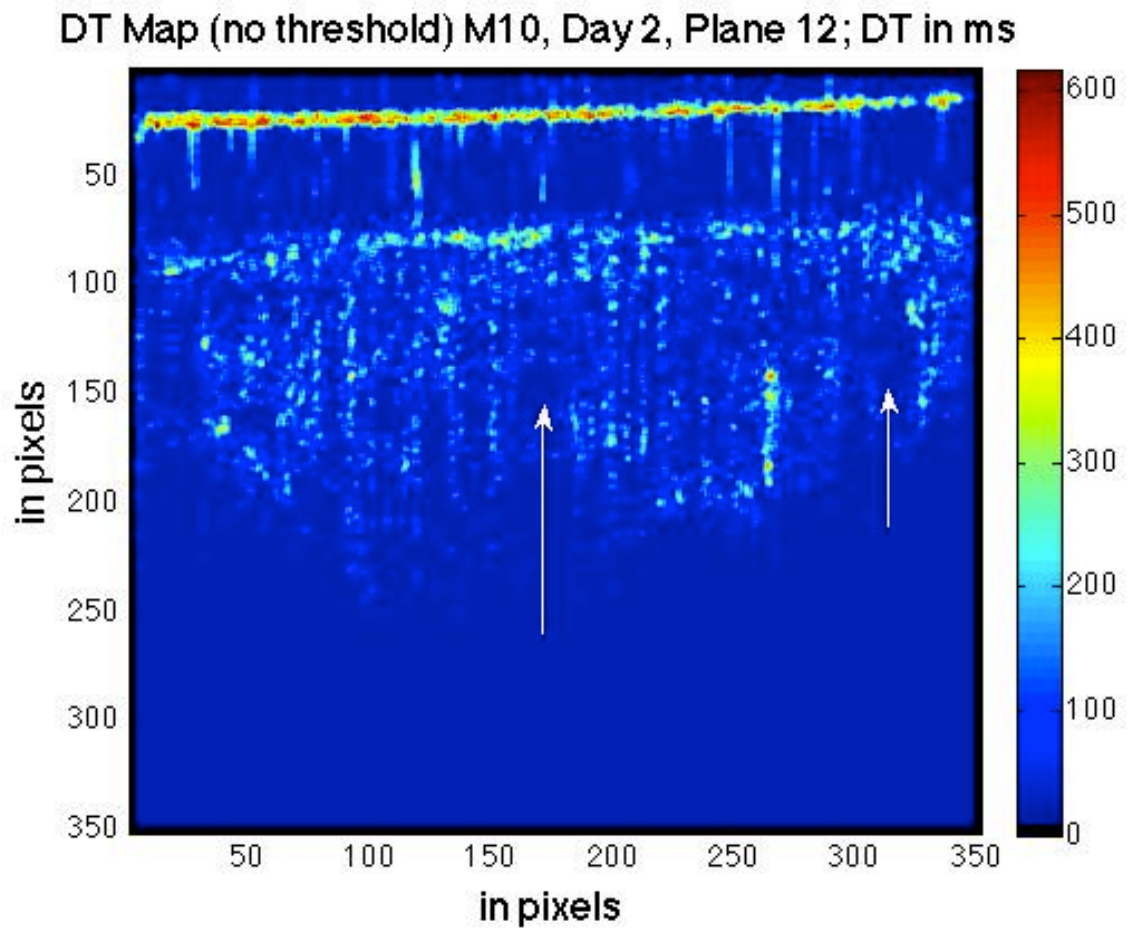

(b)

Figure 4-6: DT map (without threshold) of tumour of (a) Plane 7 of Mouse 6 on Day 1 and (b) Plane 12 of Mouse 10 on Day 2. The white arrows point out suspected blood 
vessel locations and their shadowing effect. The image height and image width are both 3 $\mathrm{mm}$.

Three approaches were used to determine the most suitable threshold DT value to exclude pixels corresponding to the location of blood vessels from the analysis of tissue response. The first method involved comparing the actual DT pixel values in the prethreshold DT maps with existing data from previous studies. Figures 4-6a and 4-6b show the pre-threshold DT maps of imaging plane 7 for Mouse 6 on Day 1 and imaging plane 12 for Mouse 10 on Day 2, respectively. For both DT maps, the tumour growth was observed near the centre of the DT map. The longest and shortest DT values measured were 617 and $20 \mathrm{~ms}$, respectively. Areas of short DT were observed to the right of the tumour for Mouse 6 and are indicated by the white arrows. Similarly, white arrows at the centre and right side of the tumour indicate short DT regions for Mouse 10. Some of these areas of short DT have a symmetrical shape and cast a streaking or shadowing effect (more pronounced in Figure 4-6a than Figure 4-6b) indicating the vessel locations. In Figure 4-6, the specific DT values at the shadowed areas ranged from 20 to 30 milliseconds. This observation is consistent with the observation of the short DT times measured in vitro; motion caused by flow will have much shorter DT than of cells in vitro [11]. The other approaches will be described in the two following sections regarding the quantitative analysis of average DT variations between possible threshold values (Section 4.2.1) and the qualitative analysis of the DT map background (Section 4.2.2), respectively.

\subsubsection{Quantitative Analysis of Thresholding}


A quantitative assessment to establish the most suitable value for thresholding all DT maps was performed. A threshold value (e.g. DT $=20 \mathrm{~ms}$ ) and a ROI (e.g. a region in the DT map of size 50 by 30 pixels) were selected for the first assessment. The pixels in the ROI with DT values less than this threshold value were assigned a value of zero so that the pixels could be excluded from further analysis. A pixel count was taken for all nonzero pixels within the ROI. The average of all the remaining nonzero DT values was then calculated. These steps were repeated for other threshold values close to these values (e.g. DT $=30,40,50$, and $60 \mathrm{~ms}$ ).

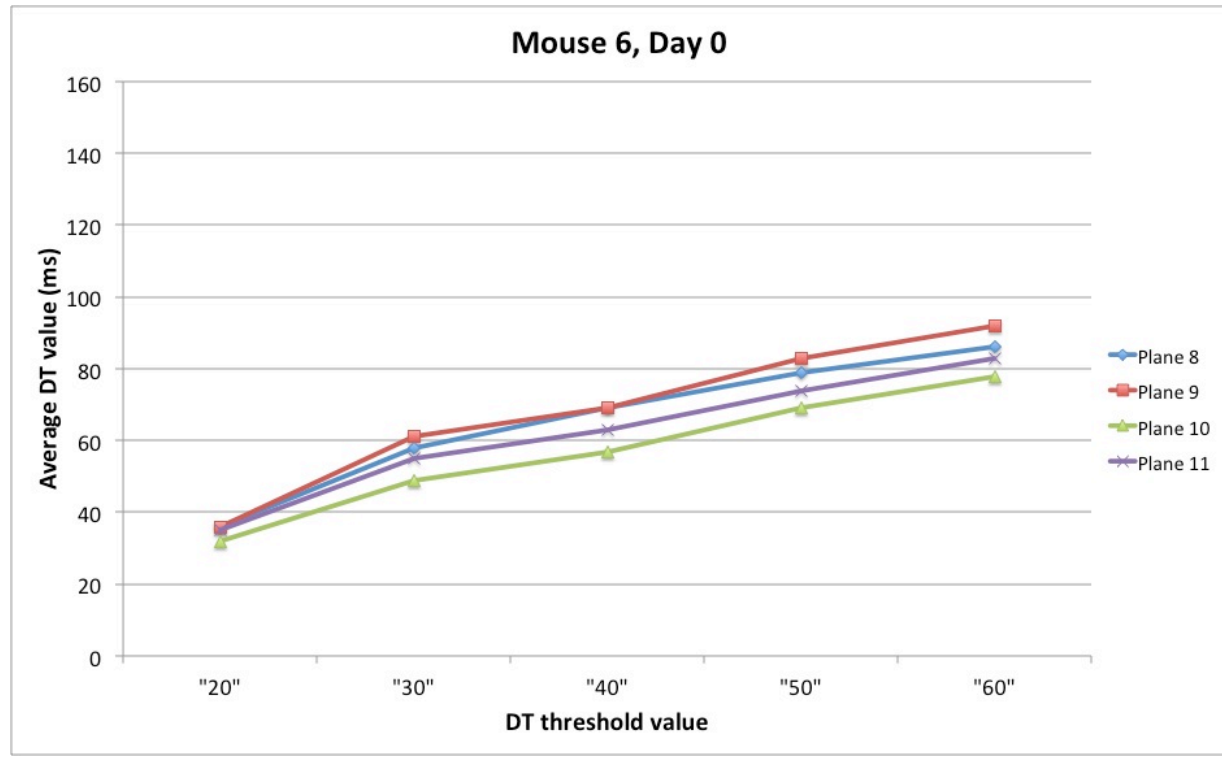

(a) 


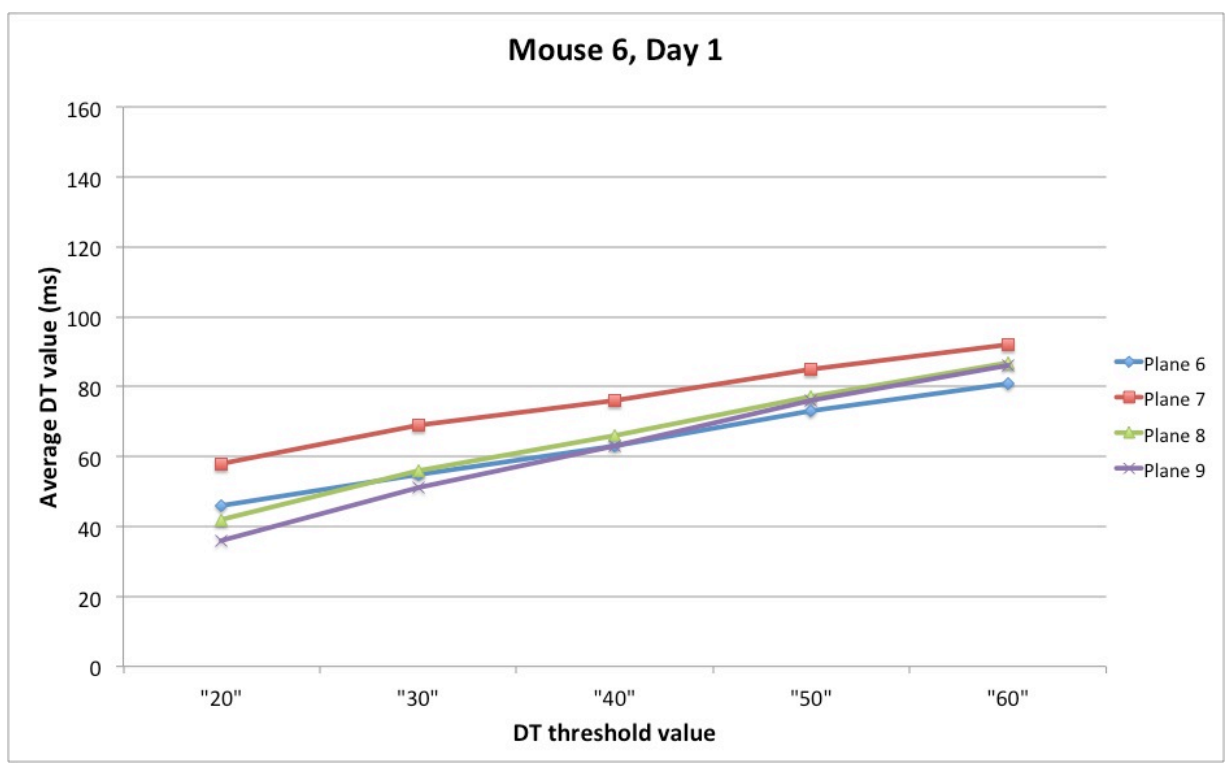

(b)

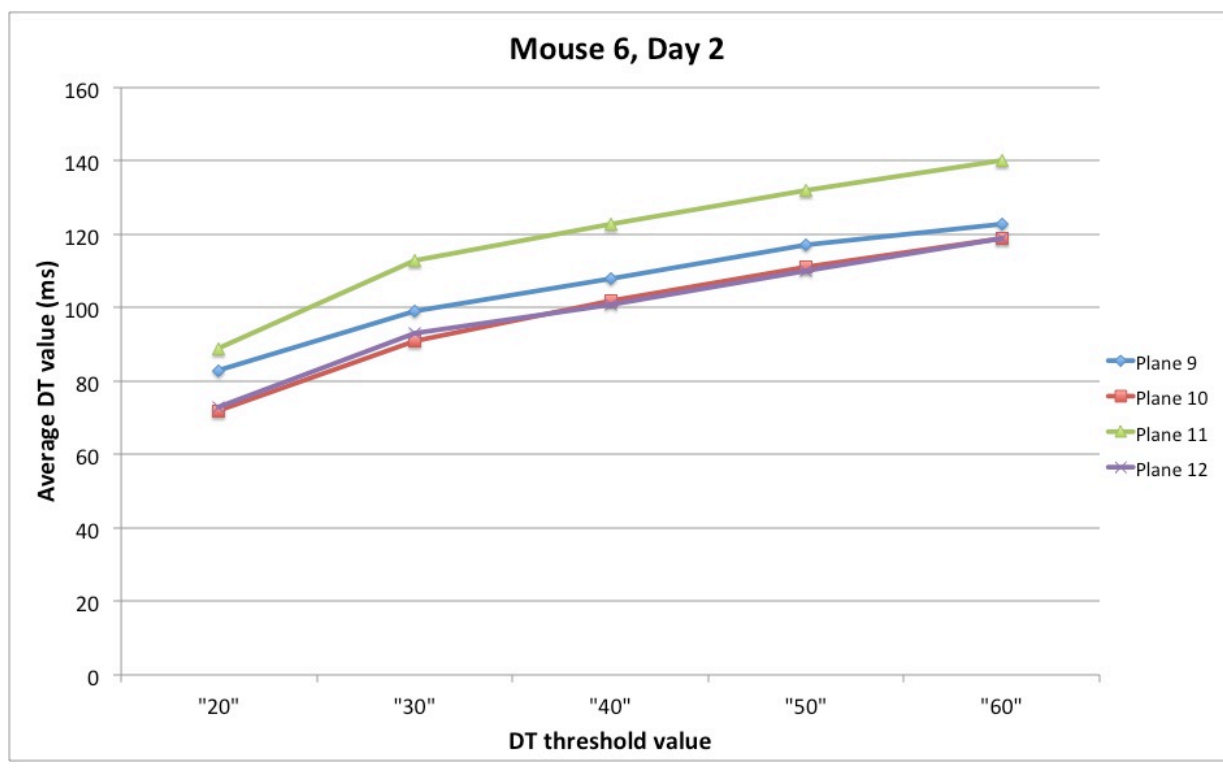

(c)

Figure 4-7: Average DT values of different thresholds (20, 30, 40, 50, and $60 \mathrm{~ms})$ for Mouse 6 for (a) Day 0; (b) Day 1; and (c) Day 2.

A ROI of size 50 by 30 pixels was chosen at the centre of the tumour in order to observe the effects of thresholding of vessels within the tumour on the ROI average DT values. Figure 4-7 shows the average tumour DT values as a function of the respective DT thresholds applied. Figure 4-7a shows the average DT values of Mouse 6 on Day 0 for planes 8 to 11 , using threshold values of 20,30,40, 50, and $60 \mathrm{~ms}$ to generate the DT 
maps of those imaging planes. This ROI of size 50 by 30 pixels was selected at pixels at the centre of the tumour with a diameter of 1-2 $\mathrm{mm}$. The pixel length and width were both $8.6 \mu \mathrm{m}$ and were consistent throughout the thesis.

Table 4-2: An excerpt of the chart data corresponding to Figure 4-7 for Mouse 6 from Day 0 to Day 2. Difference refers to the increase in average DT compared to the next longest DT threshold. The maximum pixel count based on the ROI chosen was 1500 . See Appendix E for the full summary chart.

\begin{tabular}{ccccc|ccccc}
\hline \multicolumn{6}{c|}{ Day 0 } & \multicolumn{5}{c}{ Day 1 } \\
\hline Plane & Thres & $\begin{array}{c}\text { Avg } \\
\text { DT }\end{array}$ & Diff & PixelCount & Plane & Thres & $\begin{array}{c}\text { Avg } \\
\text { DT }\end{array}$ & Diff & PixelCount \\
\hline 8 & 20 & 36 & 22 & $1500(100 \%)$ & 6 & 20 & 46 & 9 & $1500(100 \%)$ \\
& 30 & 58 & 11 & $582(38.8 \%)$ & & 30 & 55 & 8 & $1065(71.0 \%)$ \\
& 40 & 69 & 10 & $401(26.7 \%)$ & & 40 & 63 & 10 & $769(53.6 \%)$ \\
& 50 & 79 & 7 & $290(19.3 \%)$ & & 50 & 73 & 8 & $504(33.6 \%)$ \\
& 60 & 86 & & $223(14.9 \%)$ & & 60 & 81 & & $350(23.3 \%)$ \\
9 & 20 & 36 & 25 & $1500(100 \%)$ & 7 & 20 & 58 & 11 & $1500(100 \%)$ \\
& 30 & 61 & 8 & $555(37.0 \%)$ & & 30 & 69 & 7 & $1125(75.0 \%)$ \\
& 40 & 69 & 14 & $428(28.5 \%)$ & & 40 & 76 & 9 & $939(62.6 \%)$ \\
& 50 & 83 & 9 & $280(18.7 \%)$ & & 50 & 85 & 7 & $741(49.4 \%)$ \\
& 60 & 92 & & $211(14.1 \%)$ & & 60 & 92 & & $596(39.7 \%)$ \\
\hline
\end{tabular}

Table 4-2 contains a summary of the raw data used to plot the graphs in Figure 47. It was observed for all imaging planes that 1500 nonzero pixels (which was the maximum number of pixels within the ROI of $50 \times 30$ pixels) were counted when threshold of $20 \mathrm{~ms}$ was applied, thus indicating that all the pixels in the ROI had DT greater than $20 \mathrm{~ms}$. Therefore, the threshold of $20 \mathrm{~ms}$ was not suitable in segmenting out any pixels, including short DT pixels suspected as vessel locations.

The graphs in Figure 4-7 illustrate an increase of average DT with the use of a longer DT threshold value. Figures 4-7b and 4-7c plot the same data for a selection of imaging planes for Mouse 6 for Day 1 and Day 2, respectively. For many of the imaging planes, the most significant change in the average DT values between thresholds within each imaging plane was from changing the threshold from 20 to $30 \mathrm{~ms}$. This observation 
was made by noting the different slopes of the line graphs between points, as well as by the pixel count for the ROI at each threshold, as indicated in Table 4-2. The differences between the average DT values of adjacent thresholds were also calculated to show the increase of average DT from threshold to threshold. The results from observing these differences showed the greatest average DT increase between 20 to $30 \mathrm{~ms}$ for most of the imaging planes, compared to the average DT increases from thresholds 30 to $40 \mathrm{~ms}, 40$ to $50 \mathrm{~ms}$, and 50 to $60 \mathrm{~ms}$. Appendix E has the full summary chart.

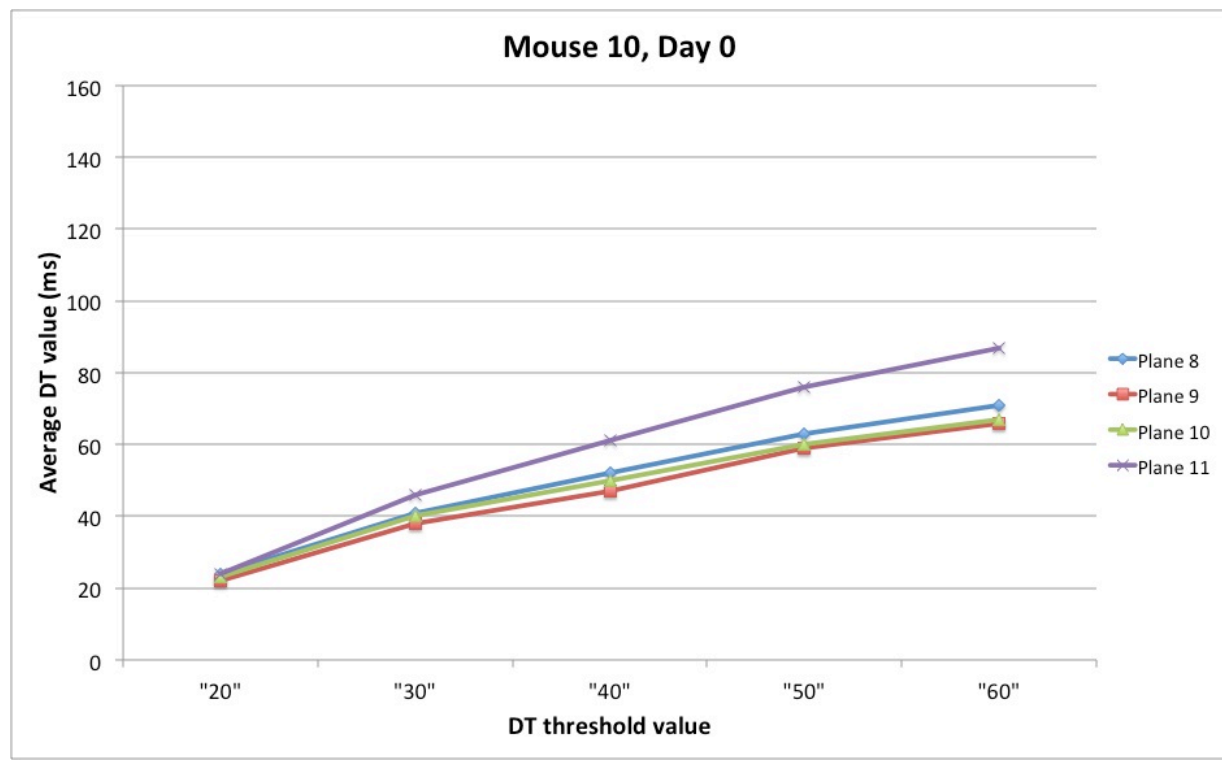

(a)

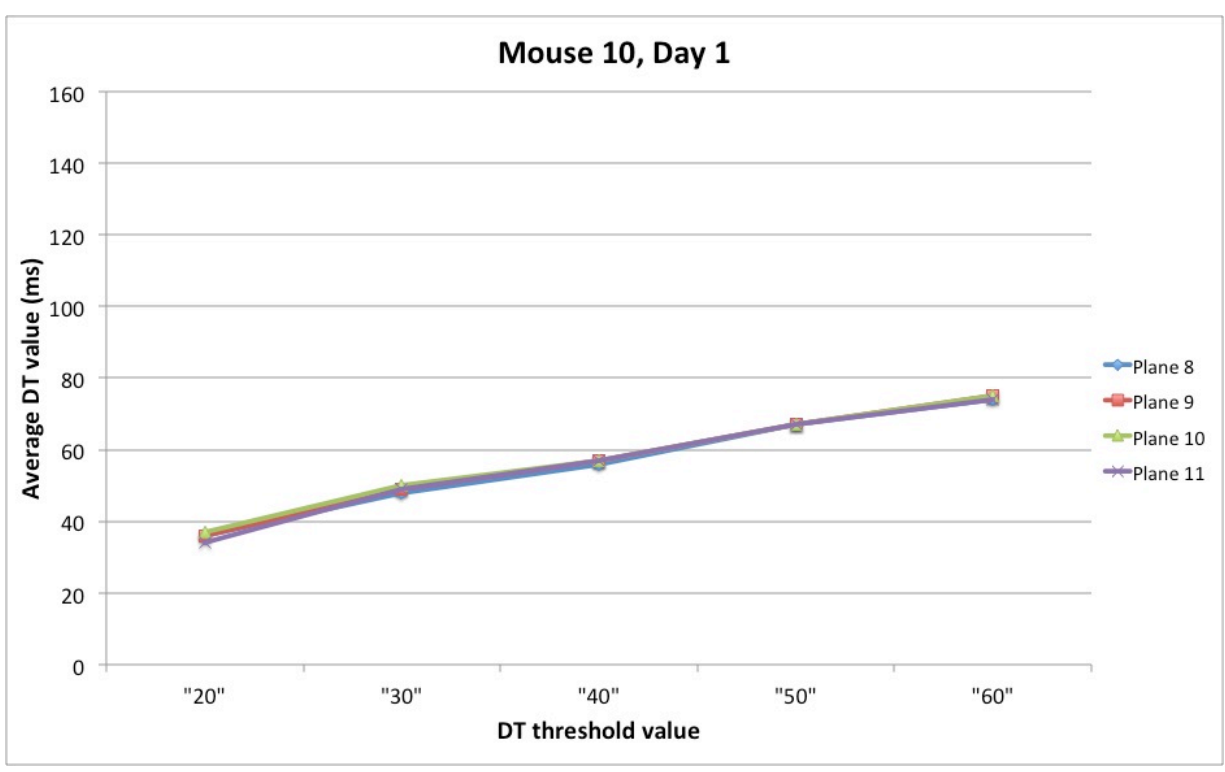




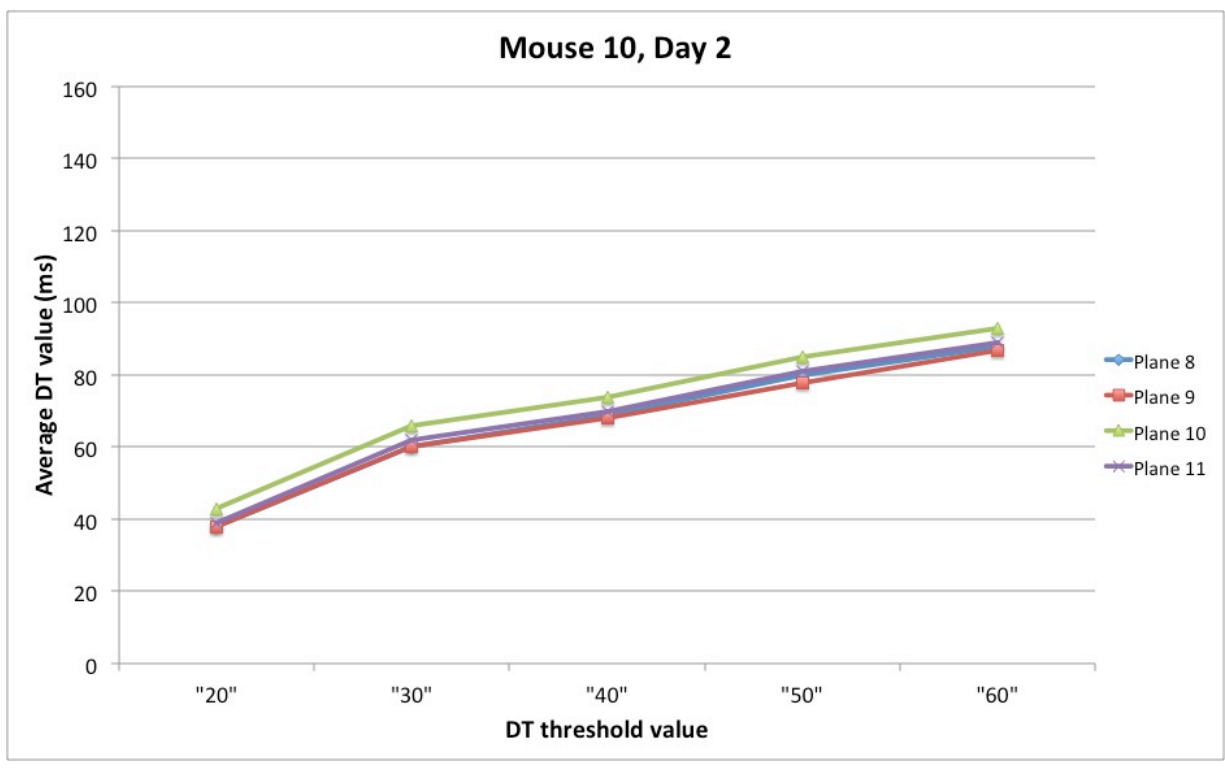

(c)

Figure 4-8: Average DT values of different thresholds (20, 30, 40, 50, and $60 \mathrm{~ms})$ for Mouse 10 for (a) Day 0; (b) Day 1; and (c) Day 2.

This process of thresholding was repeated for all the mice in the study to examine the effect of picking different thresholds on the average DT values. The line graphs for Day 0 to Day 2 for Mouse 10 are shown in Figures 4-8a to 8c. A larger ROI of 9000 pixels (which was the maximum number of pixels within the ROI of $150 \times 60$ pixels) was chosen to cover a larger tumour region for Mouse 10 than Mouse 6. The same pattern observed for Mouse 6 in Figures 4-7a to 4-7c was seen in Mouse 10, including the increase of average DT for longer DT threshold values, with the greatest variation between thresholds of 20 and $30 \mathrm{~ms}$. 
Table 4-3: An excerpt of the chart data corresponding to Figure 4-8 for Mouse 10 from Day 0 to Day 2. Difference refers to the increase in average DT compared to the next longest DT threshold. The maximum pixel count based on the ROI chosen was 9000. See Appendix E for the full summary chart.

\begin{tabular}{ccccc|ccccc}
\hline & \multicolumn{4}{c|}{ Day 0 } & \multicolumn{5}{c}{ Day 1 } \\
\hline Plane & Thres & $\begin{array}{c}\text { Avg } \\
\text { DT }\end{array}$ & Diff & PixelCount & Plane & Thres & $\begin{array}{c}\text { Avg } \\
\text { DT }\end{array}$ & Diff & PixelCount \\
\hline 10 & 20 & 23 & 17 & $9000(100 \%)$ & 10 & 20 & 37 & 13 & $9000(100 \%)$ \\
& 30 & 40 & 10 & $852(9.47 \%)$ & & 30 & 50 & 7 & $4709(52.3 \%)$ \\
& 40 & 50 & 10 & $308(3.42 \%)$ & & 40 & 57 & 10 & $3165(35.2 \%)$ \\
& 50 & 60 & 7 & $116(1.23 \%)$ & & 50 & 67 & 8 & $1804(20.0 \%)$ \\
\hline 11 & 60 & 67 & & $54(0.60 \%)$ & & 60 & 75 & & $1095(12.2 \%)$ \\
& 20 & 24 & 22 & $9000(100 \%)$ & 11 & 20 & 34 & 15 & $9000(100 \%)$ \\
\hline & 30 & 46 & 15 & $1003(11.14 \%)$ & & 30 & 49 & 8 & $4066(45.2 \%)$ \\
& 40 & 61 & 15 & $442(4.91 \%)$ & & 40 & 57 & 10 & $2636(29.3 \%)$ \\
& 50 & 76 & 11 & $238(2.64 \%)$ & & 50 & 67 & 7 & $1528(17.0 \%)$ \\
\hline & 60 & 87 & & $161(1.79 \%)$ & & 60 & 74 & & $976(10.8 \%)$ \\
\hline
\end{tabular}

Table 4-3 shows the summary chart of all the average DT values for all thresholds used for Mouse 10, as well as the respective pixel counts of the ROI used. See Appendix E for the full summary chart. The differences between the average DT of neighbouring thresholds were also recorded to indicate the extent of the average DT increase from threshold to threshold. Similar to the results for Mouse 6, the average DT variations between thresholds of 20 to $30 \mathrm{~ms}$ was the greatest for most of the imaging planes. Based on this analysis, the DT threshold value of $30 \mathrm{~ms}$ was chosen for segmentation.

\subsubsection{Qualitative Analysis of Thresholding}




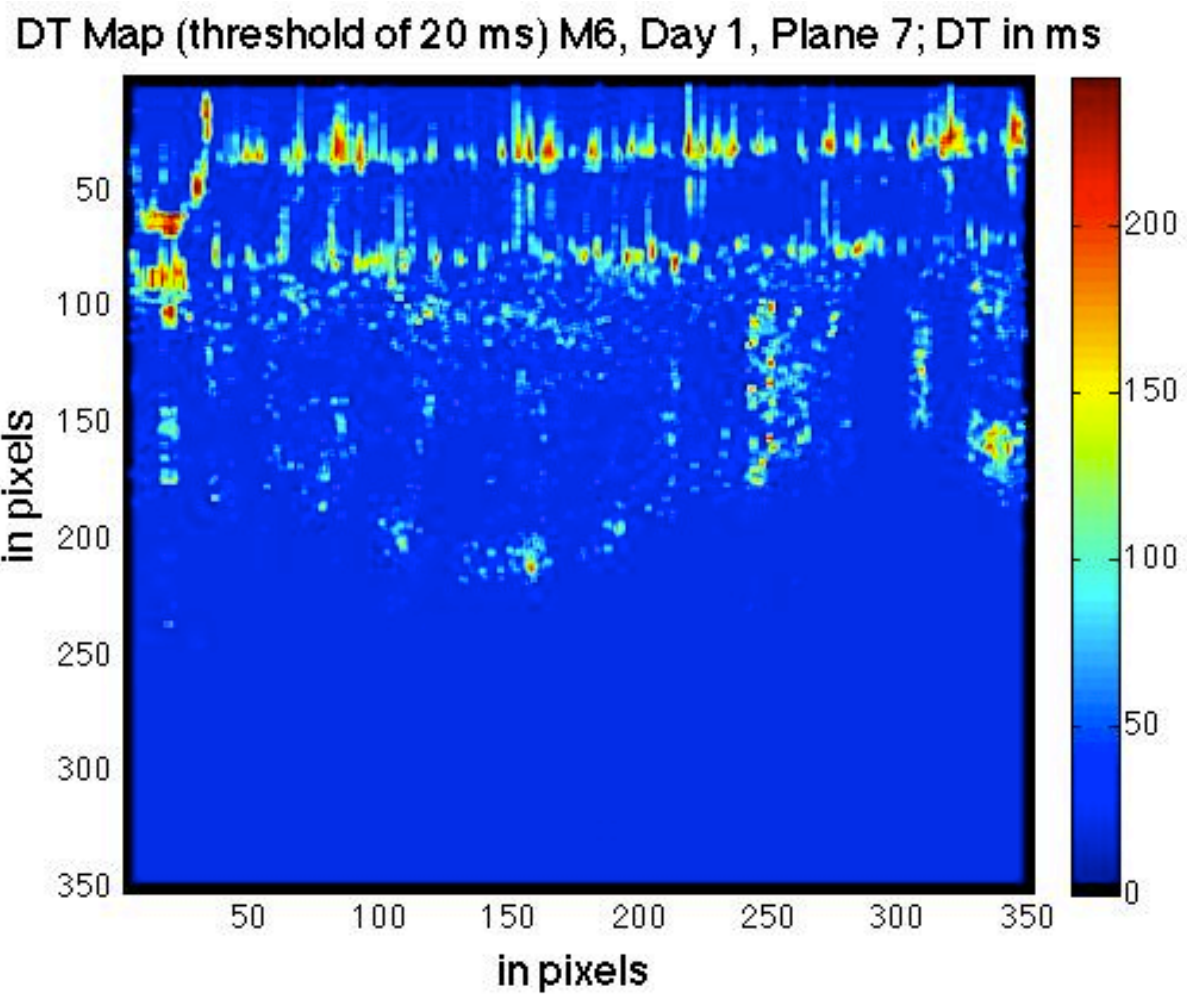

(a)

DT Map (threshold of $30 \mathrm{~ms}$ ) M6, Day 1, Plane 7; DT in ms

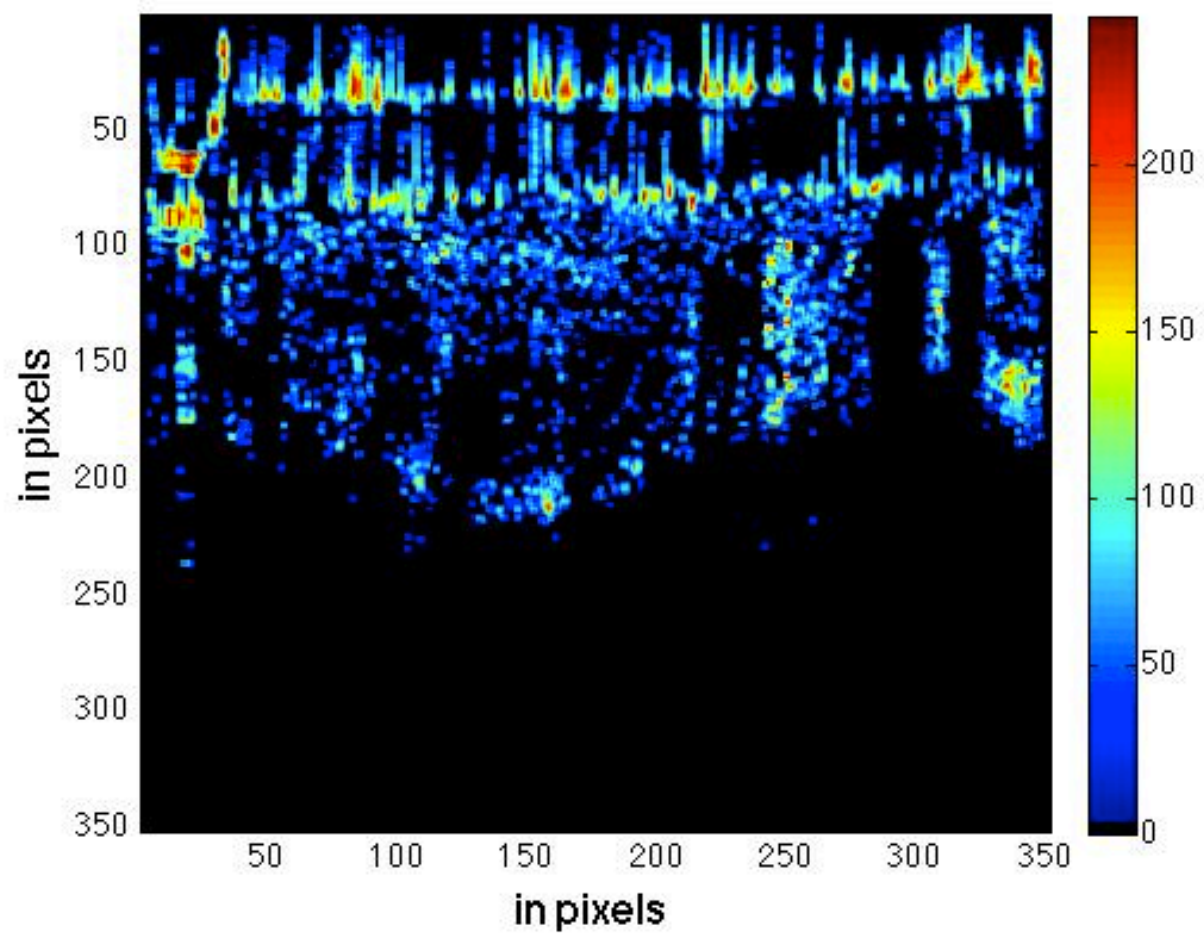

(b) 


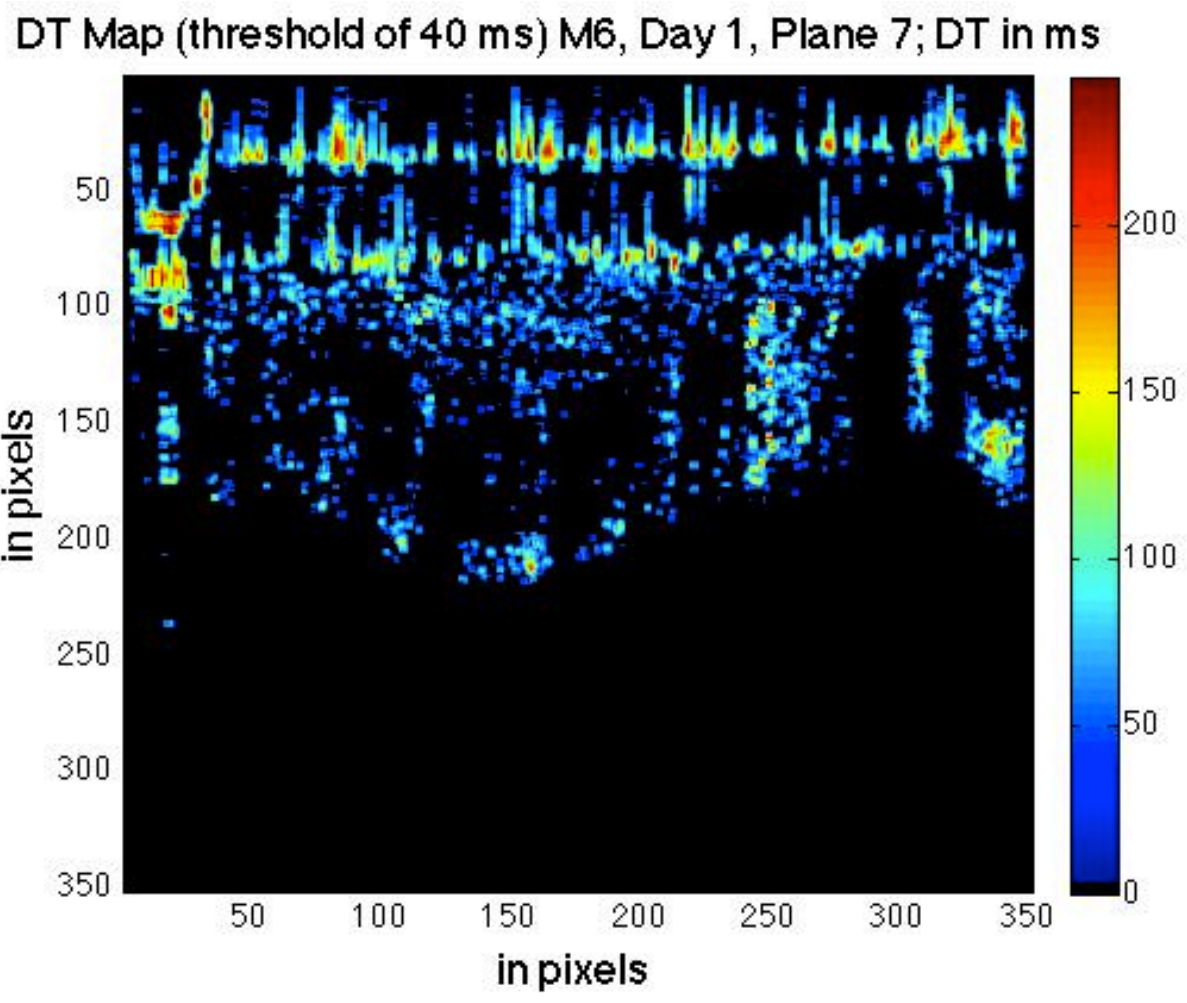

(c)

DT Map (threshold of $50 \mathrm{~ms}$ ) M6, Day 1, Plane 7; DT in ms

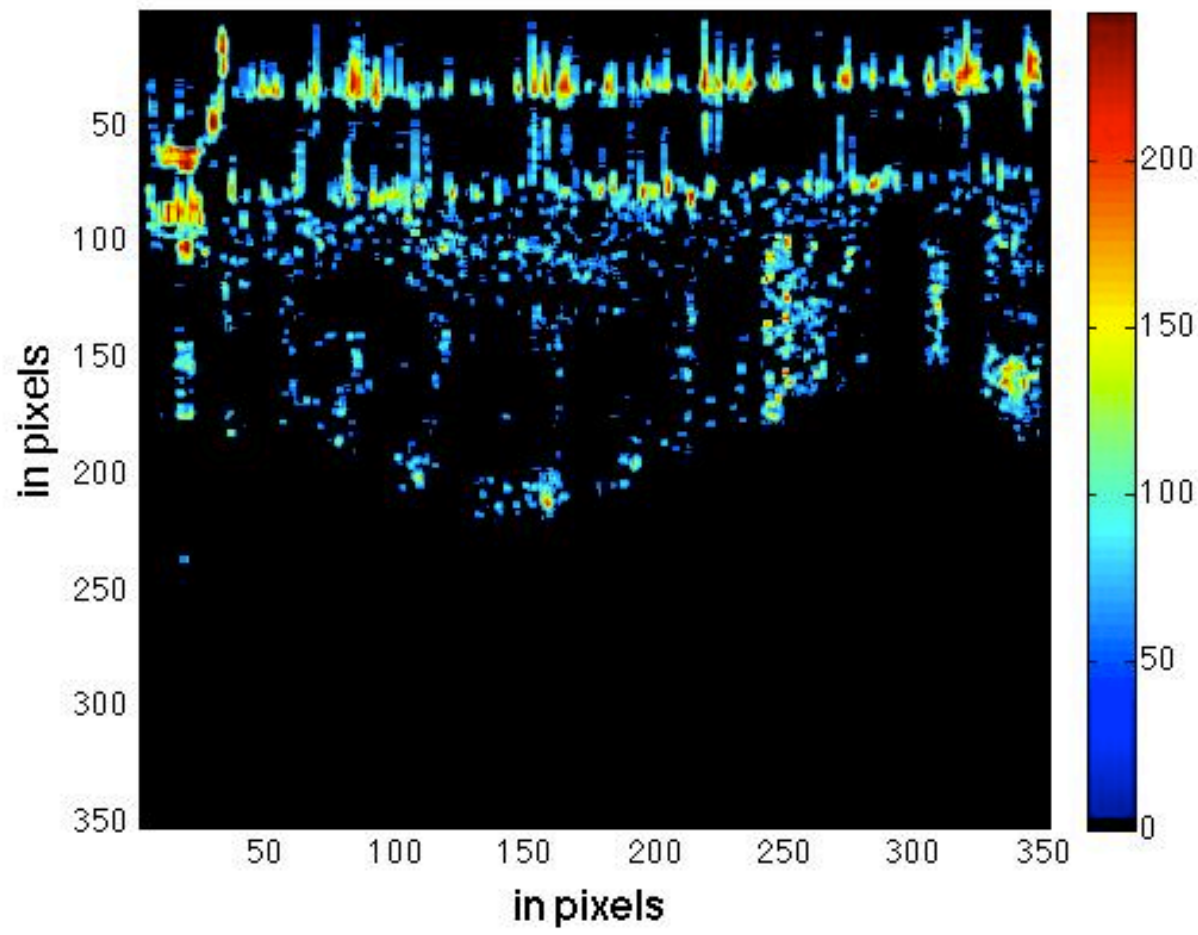

(d) 


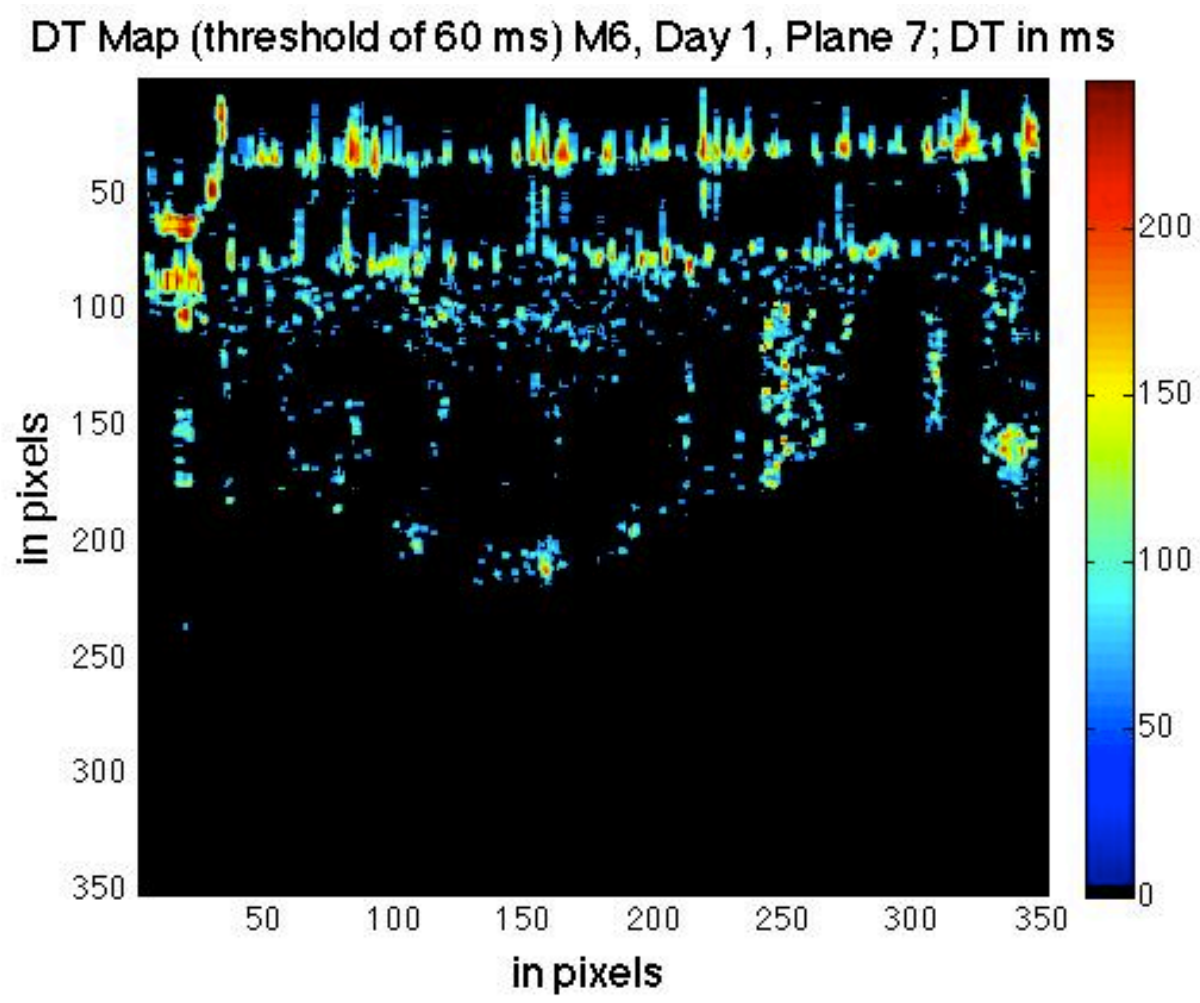

(e)

Figure 4-9: (a) DT maps for Mouse 6, Day 1, Plane 7 with a threshold of $20 \mathrm{~ms}$; (b) threshold of $30 \mathrm{~ms}$; (c) threshold of $40 \mathrm{~ms}$; (d) threshold of $50 \mathrm{~ms}$; (e) threshold of $60 \mathrm{~ms}$. The image height and image width are both $3 \mathrm{~mm}$ for all images.

The third approach used to determine the best suitable threshold value involved the qualitative assessment of the background in the DT maps. The thresholding algorithm used in the previous analysis involved zeroing all pixels in the selected ROI that were below the selected threshold value. Figures 4-9a to 4-9e illustrate the DT maps with threshold values of $20,30,40,50$, and $60 \mathrm{~ms}$ for Mouse 6 . The ROI for the thresholding algorithm for these DT maps was the entire image (350 by 350 pixels). 
DT Map (threshold of $20 \mathrm{~ms}$ ) M10, Day 2, Plane 12; DT in $\mathrm{ms}$

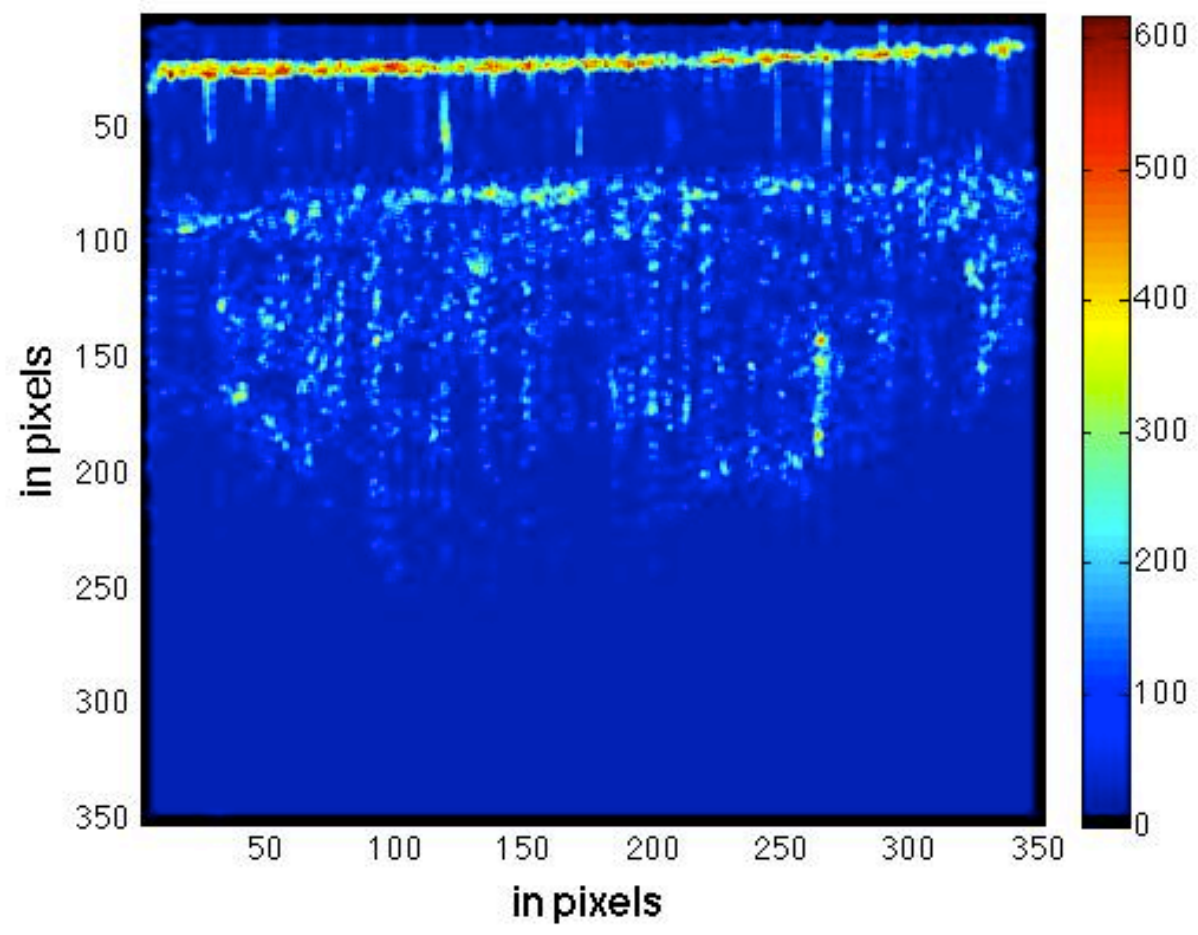

(a)

DT Map (threshold of $30 \mathrm{~ms}$ ) M10, Day 2, Plane 12; DT in $\mathrm{ms}$

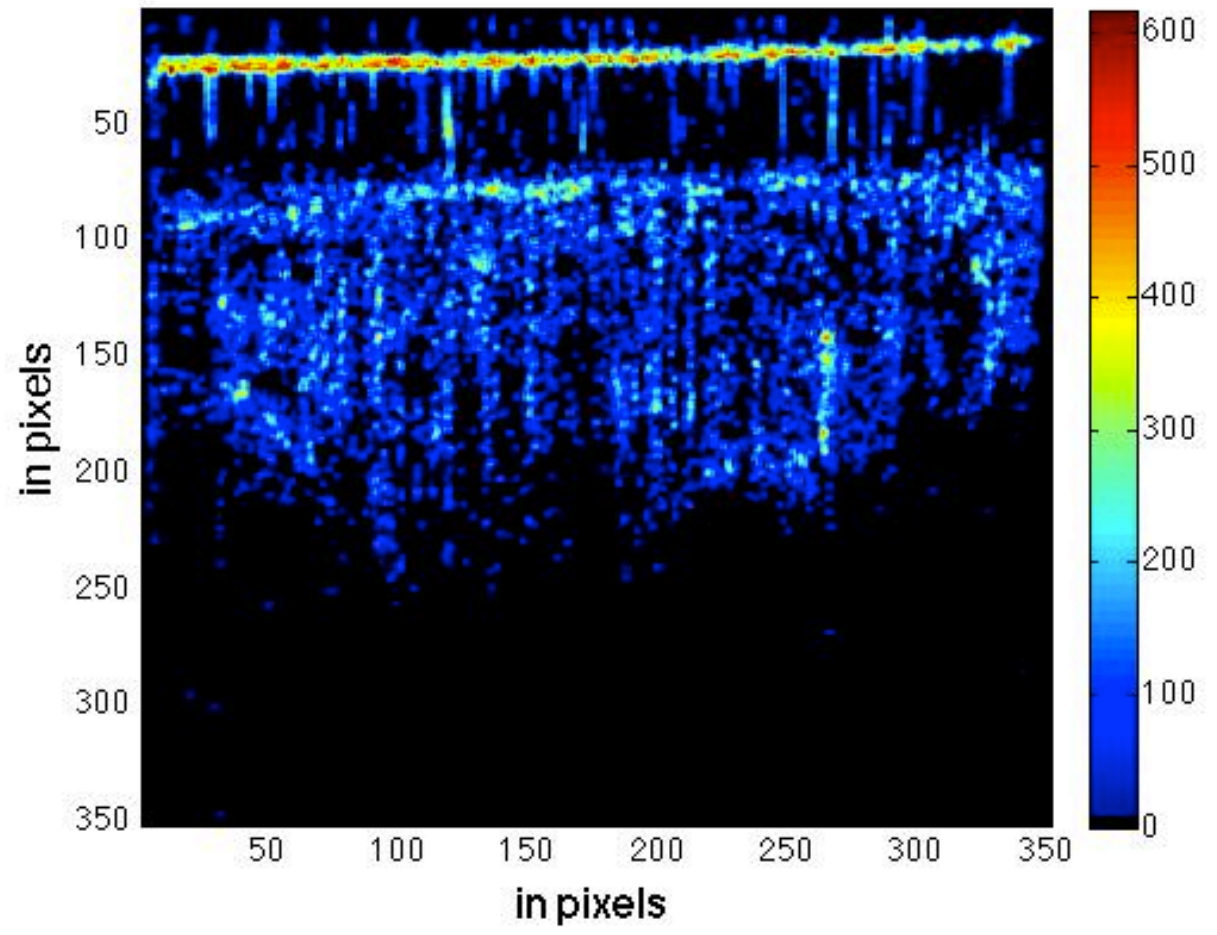

(b) 
DT Map (threshold of $40 \mathrm{~ms}$ ) M10, Day 2, Plane 12; DT in $\mathrm{ms}$

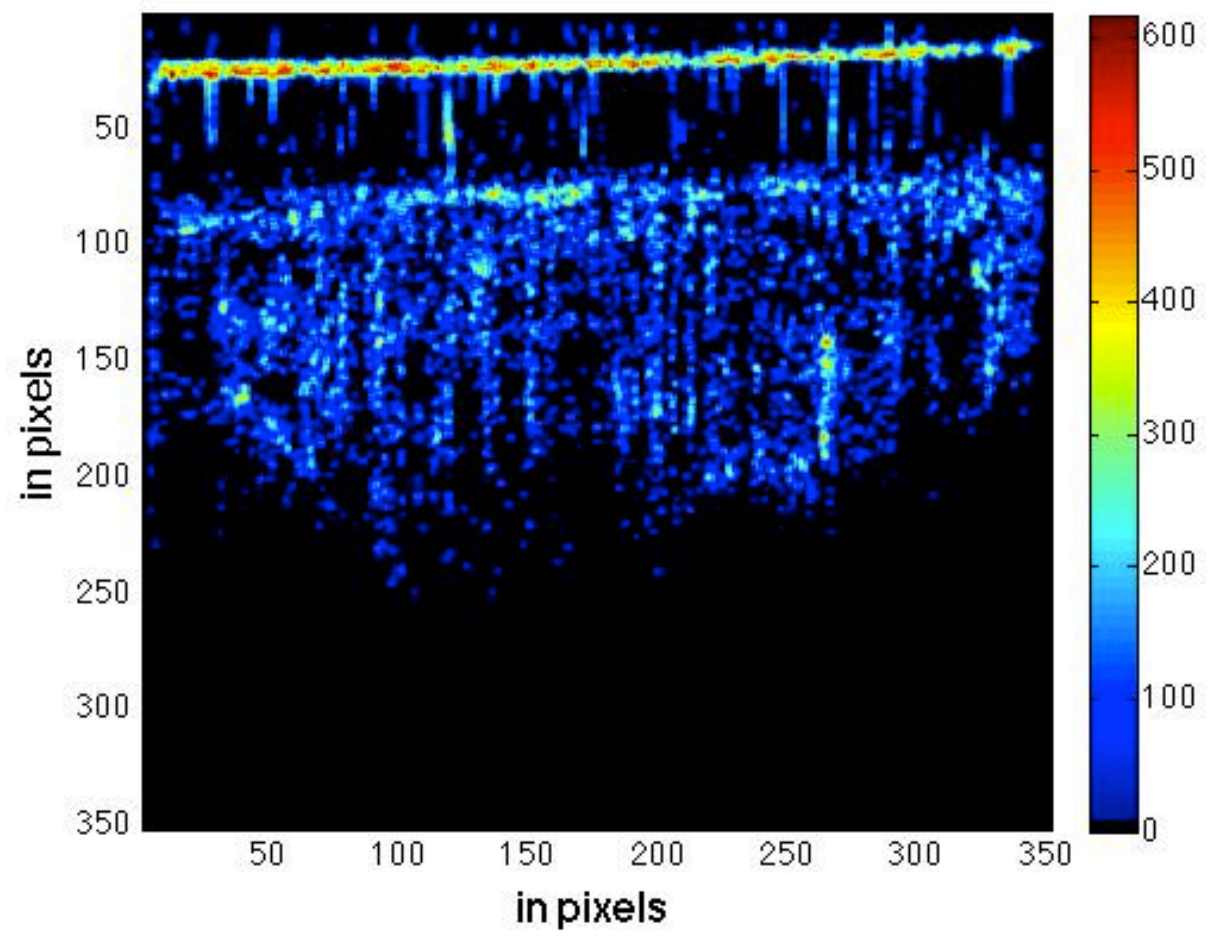

(c)

DT Map (threshold of $50 \mathrm{~ms}$ ) M10, Day 2, Plane 12; DT in $\mathrm{ms}$

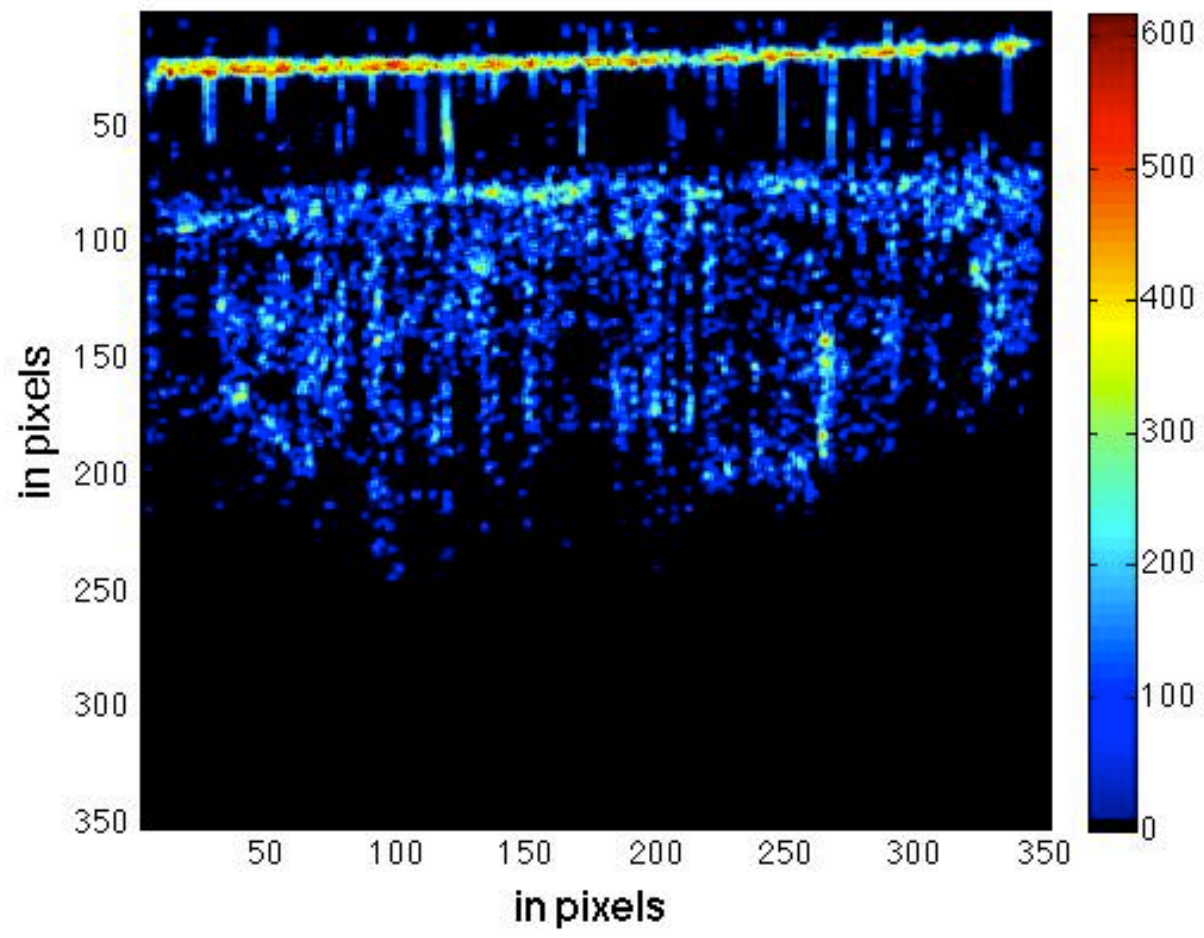

(d) 


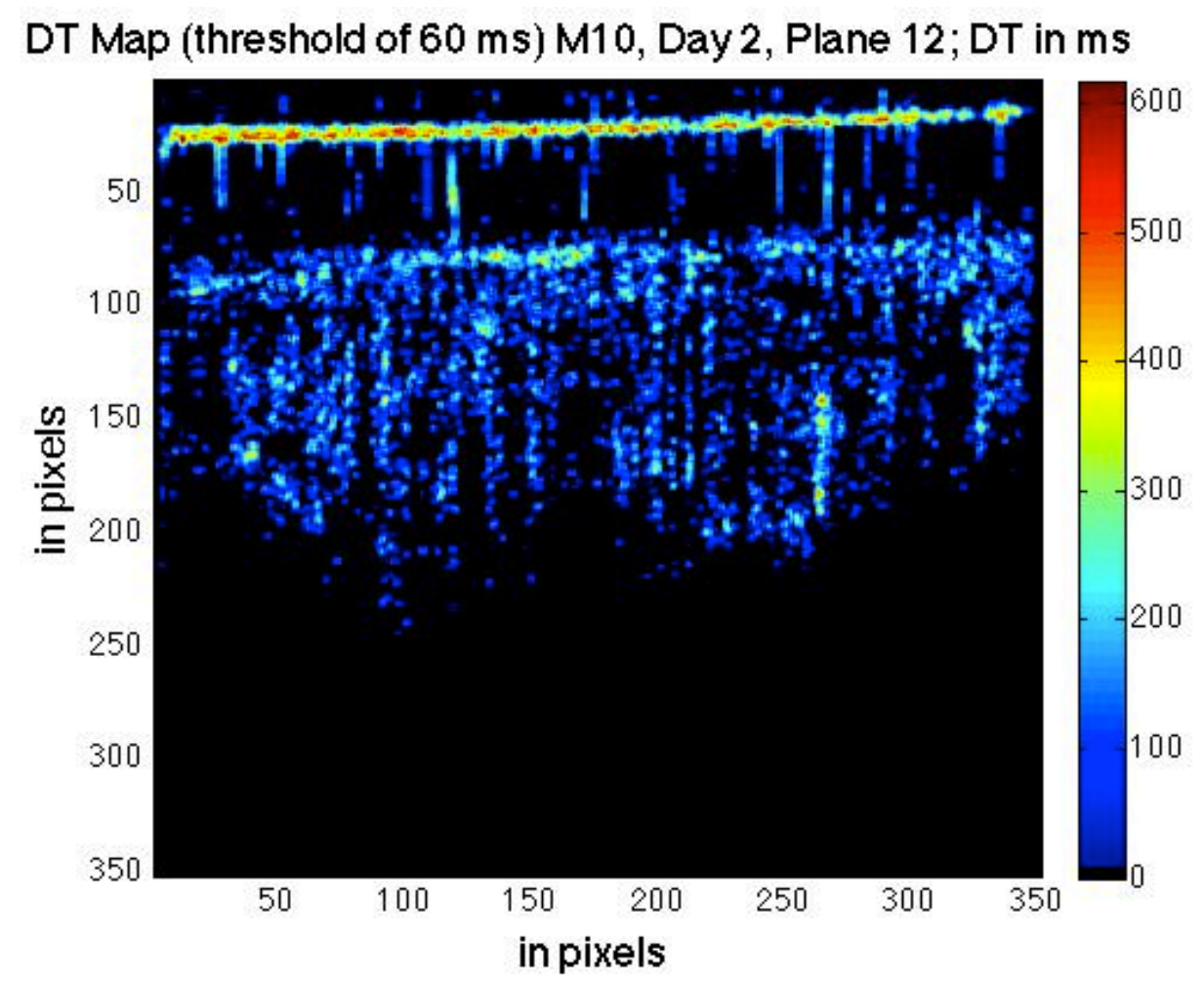

(e)

Figure 4-10: (a) DT maps for Mouse 10, Day 2, Plane 12 with a threshold of $20 \mathrm{~ms}$; (b) threshold of $30 \mathrm{~ms}$; (c) threshold of $40 \mathrm{~ms}$; (d) threshold of $50 \mathrm{~ms}$; (e) threshold of $60 \mathrm{~ms}$. The image height and image width are both $3 \mathrm{~mm}$ for all images.

With the modified colour map setting, any black pixels observed indicated zeroed pixels for the specific threshold value applied. A distinct colour change in the DT map background was observed for Figure 4-9a (threshold of $20 \mathrm{~ms}$ ) and Figure 4-9b (threshold of $30 \mathrm{~ms}$ ). The colour change between Figures 4-9b to 4-9e was less apparent. The pixels that changed to black correlated to areas of blood vessels, whereas the pixels that did not change to black correlated to non-vessel locations of DT that was longer than the threshold value. This result showed that a threshold of $30 \mathrm{~ms}$ was capable of segmenting out vessel pixels, while preserving non-vessel pixels of longer DT. The same thresholding algorithm was also applied for Mouse 10 and similar DT map results are shown in Figure 4-10. In both cases, using the threshold of $30 \mathrm{~ms}$ provided images for 
which the dark areas were well-defined and predominantly circular areas, as would be expected in the presence of blood vessels. It is not yet known what these pixels represent, but they could represent pixels for which the signal originates predominantly from noncellular regions (such as the tumour interstitium, lymphatics, etc.) [50]. The elimination of blood vessels with a threshold of $30 \mathrm{~ms}$ provides the initial segmentation of pixels due to blood flow, while retaining pixels associated with locations within the tumour interstitium.

The previous in vitro study presented tumour cells to have DT values greater than $100 \mathrm{~ms}$ [11]. Speckle variance experiments revealed the possibility of blood vessel locations at shadowed areas. By utilizing the previous knowledge, the observations of 1) the actual DT values at pixels with the shadowing effect, 2) average DT variations, and 3) DT map background changes have confirmed the threshold of $30 \mathrm{~ms}$ as the best candidate for segmentation. All subsequent results presented in this thesis with thresholding will have a threshold value of $30 \mathrm{~ms}$.

\subsection{Decorrelation Time Analysis}

Decorrelation time analysis refers to the assessment of average DT values over selected ROIs to determine any suitable trends of DT change as a function of time. The DT analysis involved comparing the average DT values of adjacent imaging planes for different ROIs (as an indication of inter-tumour variability), as well as comparing the average DT values for control and treated mice for different ROIs (as an indicator of tumour response). 


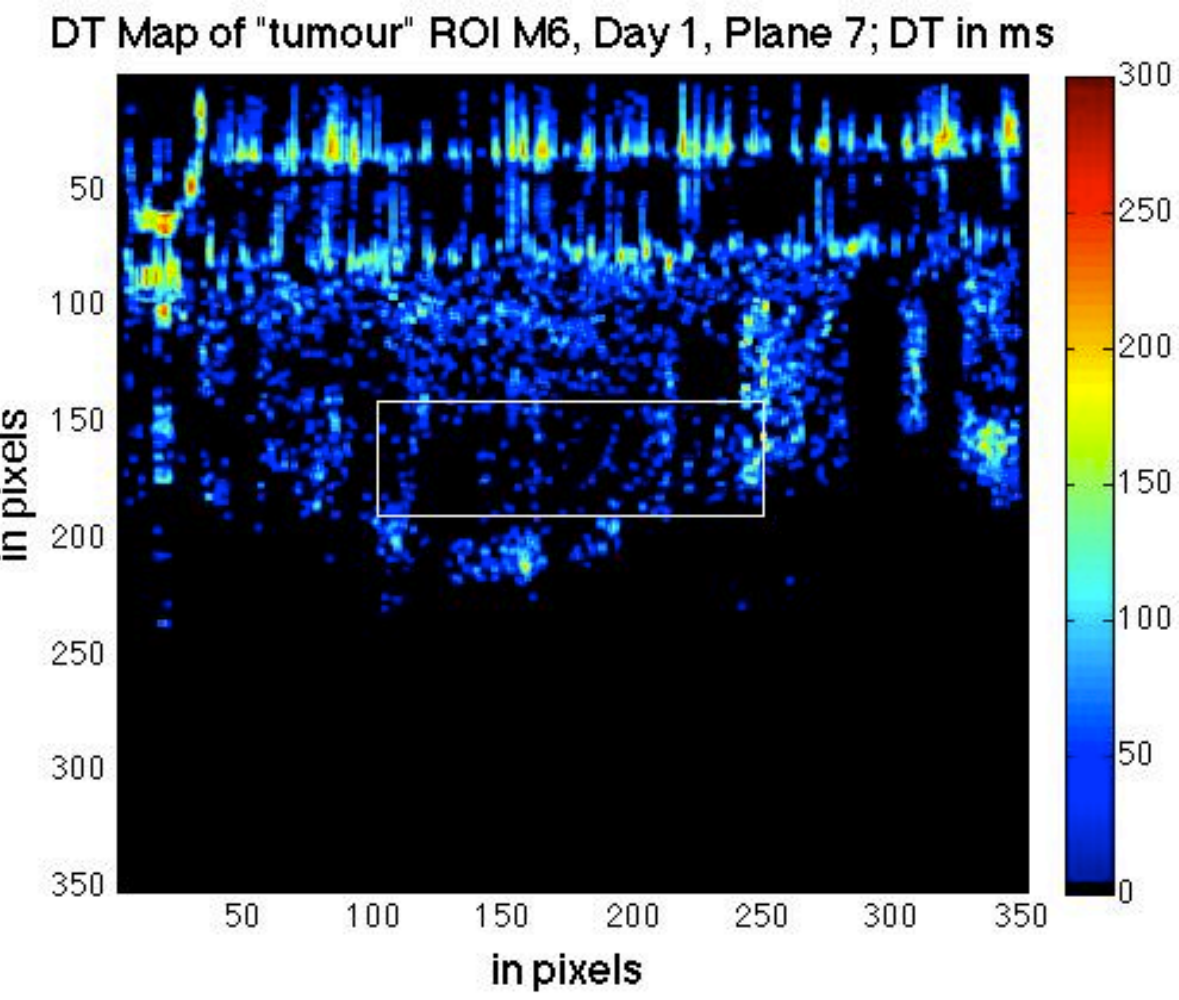

(a)

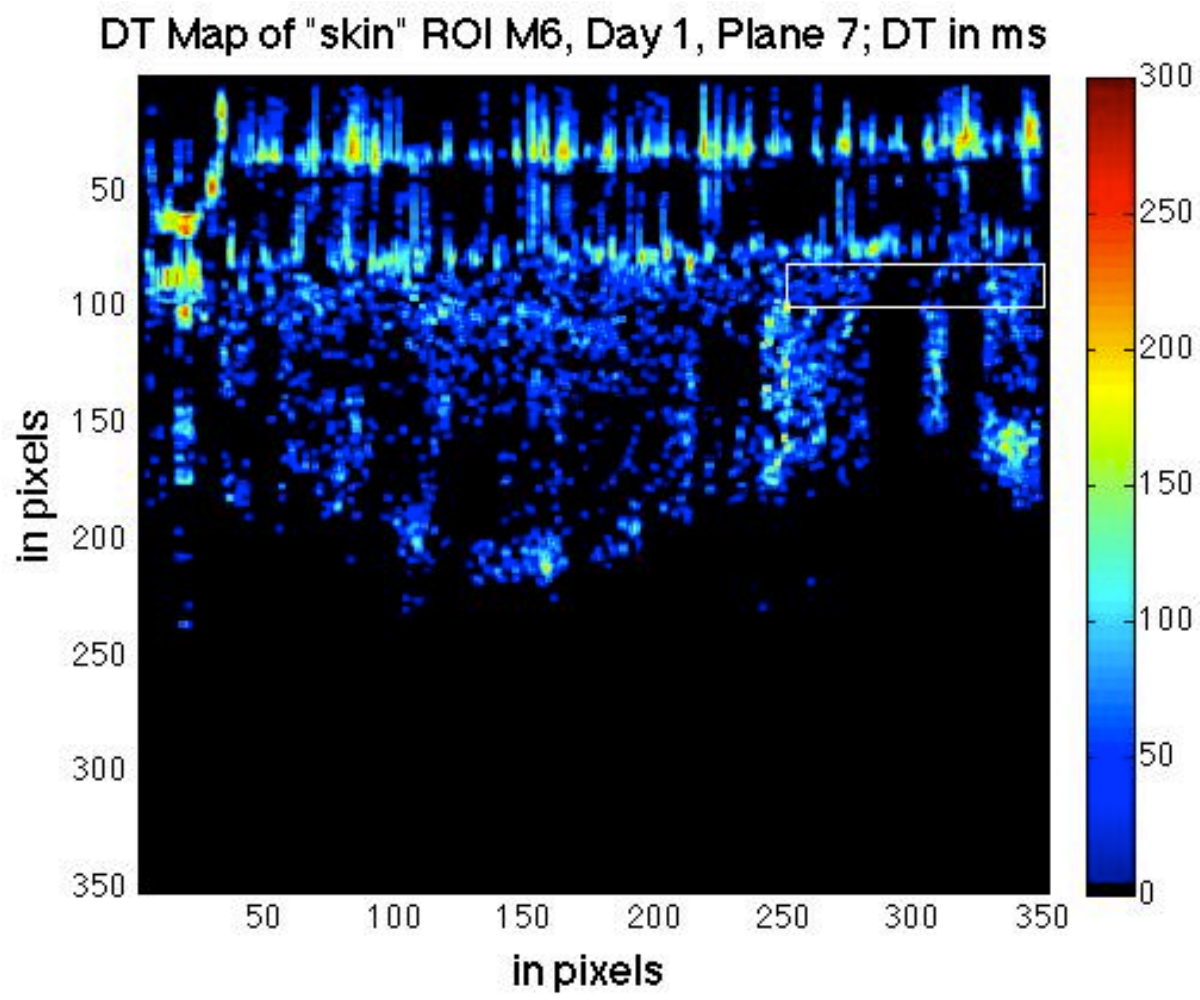

(b) 


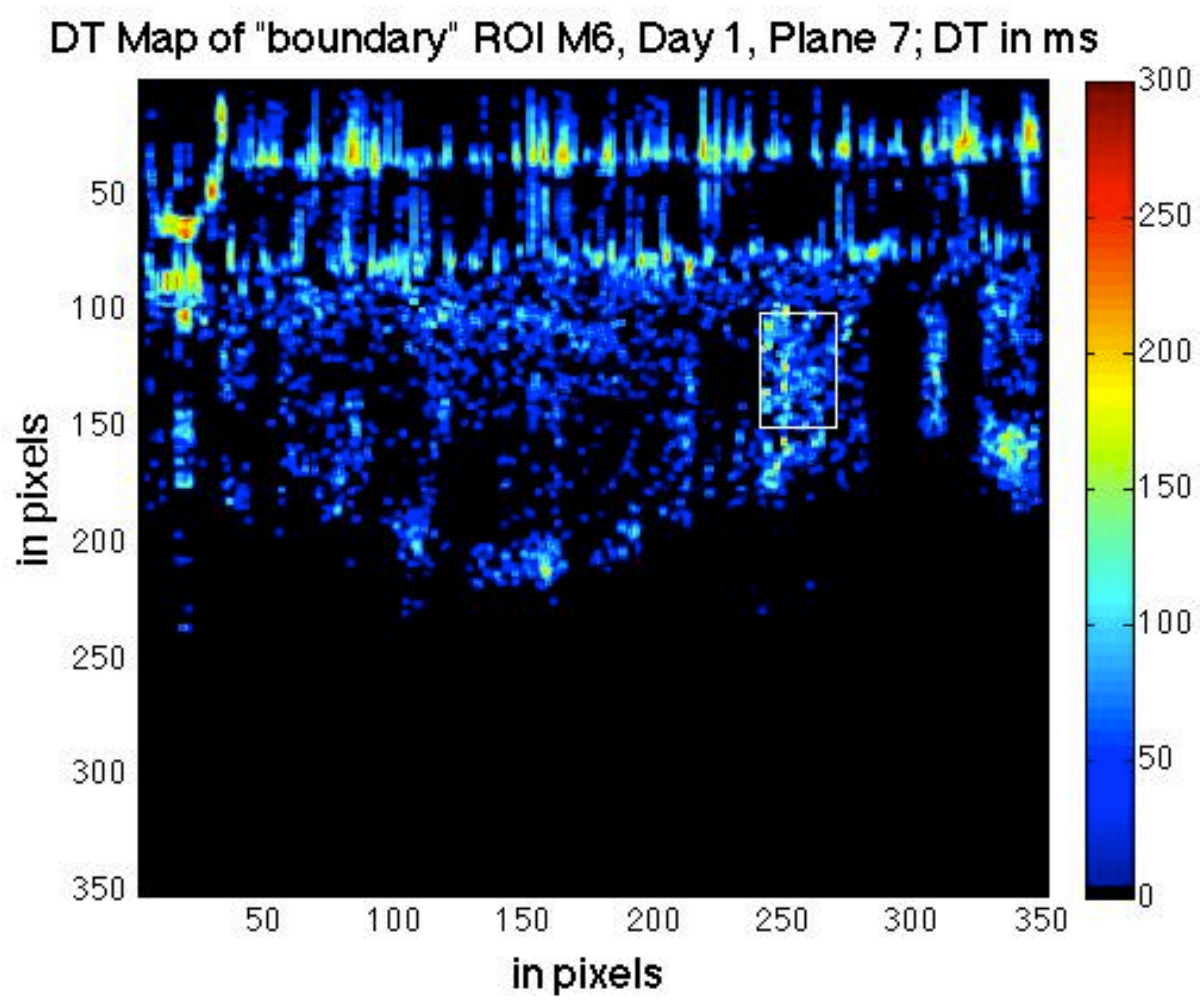

(c)

Figure 4-11: DT maps (with threshold of $30 \mathrm{~ms}$ ) for Mouse 6, Plane 7 on Day 1 with white boxes indicating (a) "tumour" ROI; (b) "skin" ROI; and (c) "boundary" ROI. The image height and image width are both $3 \mathrm{~mm}$ for all images.

\subsubsection{Decorrelation Time Averages Across Imaging Planes}

Due to the proximity of the imaging planes (each plane was separated by $25 \mu \mathrm{m}$ ), it was of interest to map out average DT values for ROIs of adjacent imaging planes to observe the average DT changes throughout the tumour. The average DT values were measured for each of the 26 imaging planes for Mouse 6 for Day 0 to Day 2 with thresholding. Three ROIs were chosen for Mouse 6, and they were labeled as "tumour" (a 60 by 150 pixel ROI near the centre of the tumour), "skin" (a 20 by 100 pixel ROI outside the tumour, near the glass coverslip of the window chamber), and "boundary" (a 50 by 30 pixel ROI at the tumour boundary). Figures $4-11$ a to $4-11 \mathrm{c}$ show the three ROI 
locations for plane 7 of Mouse 6 on Day 1. These steps were repeated for plane 12 of Mouse 10 on Day 2 and are shown in Figures 4-12a to 4-12c.

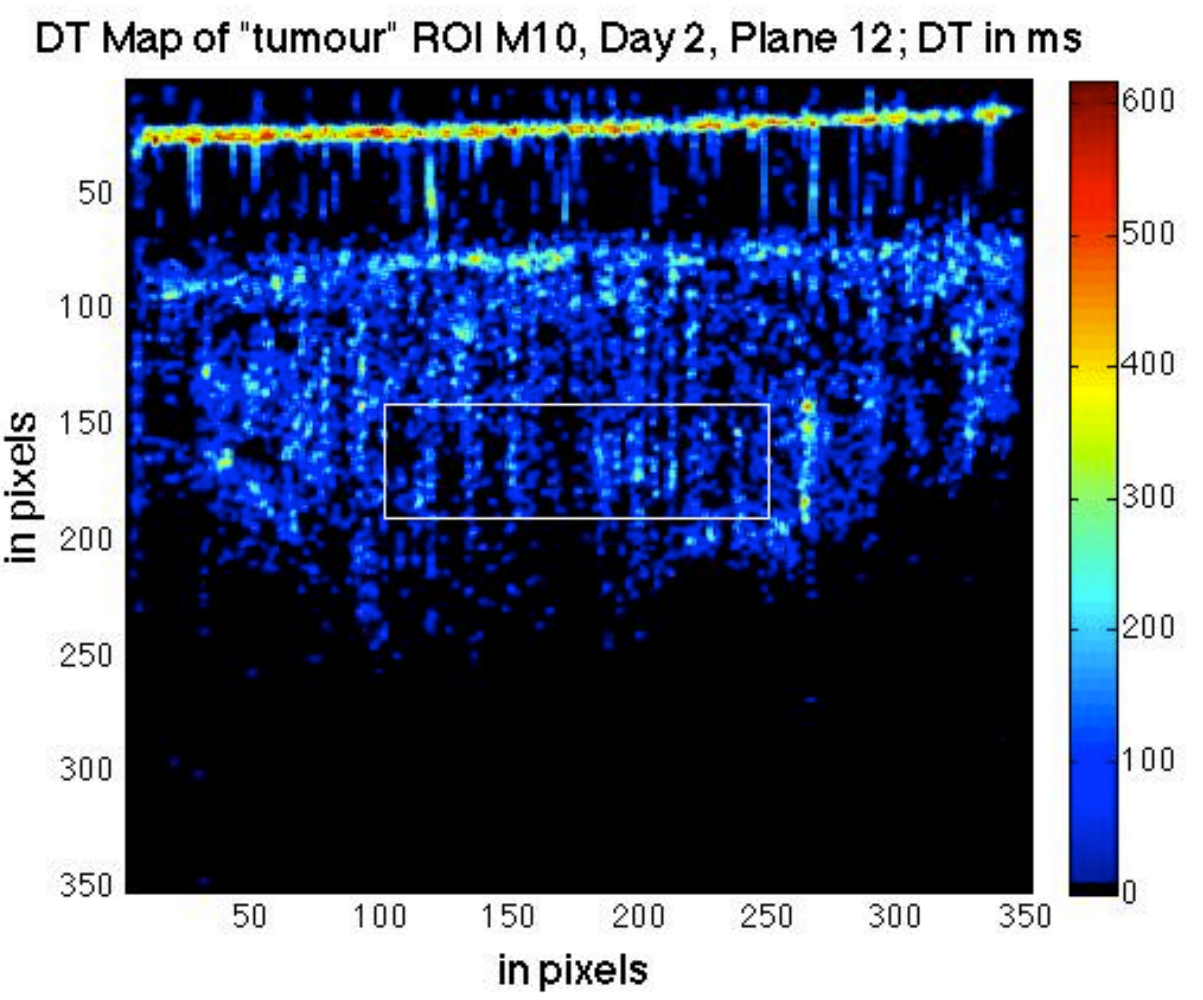

(a) 


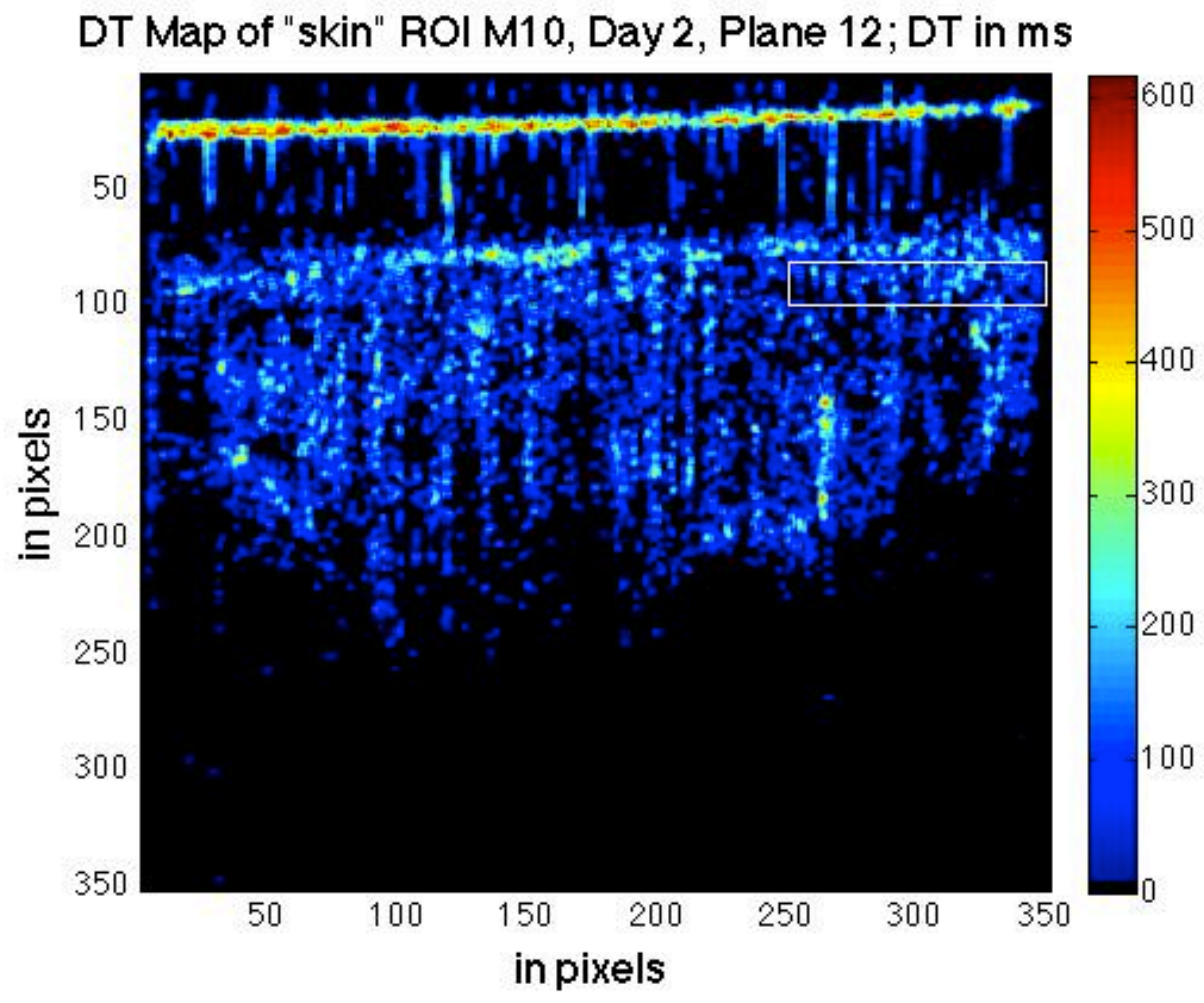

(b)

DT Map of "boundary" ROI M10, Day 2, Plane 12; DT in ms

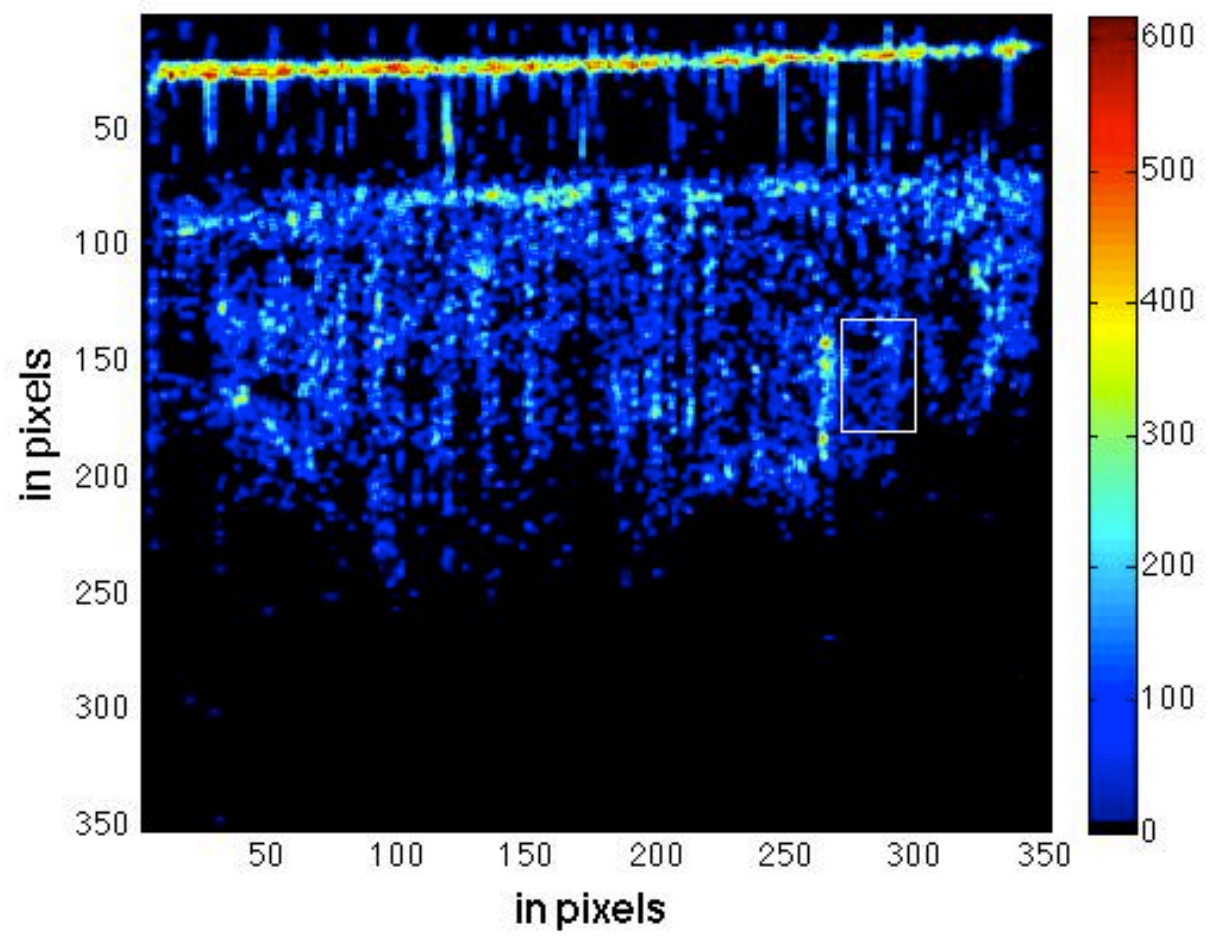

(c) 
Figure 4-12: DT maps (with threshold of $30 \mathrm{~ms}$ ) for Mouse 10, Plane 12 on Day 2 with white boxes indicating (a) "tumour" ROI; (b) "skin" ROI; and (c) "boundary" ROI. The image height and image width are both $3 \mathrm{~mm}$ for all images.

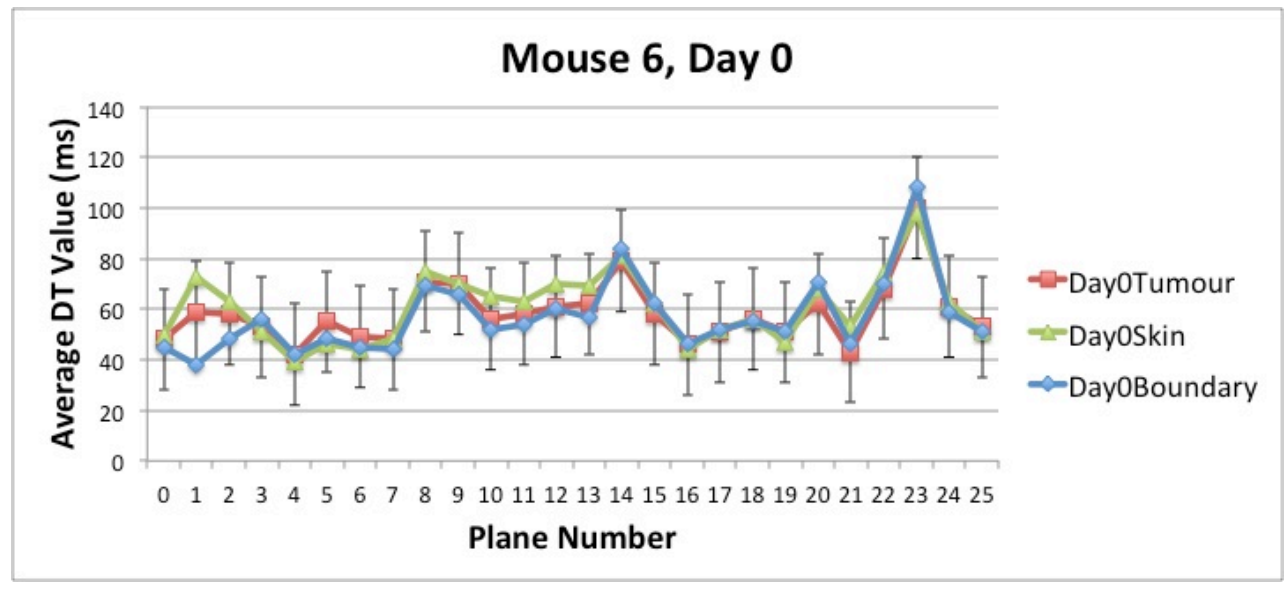

(a)

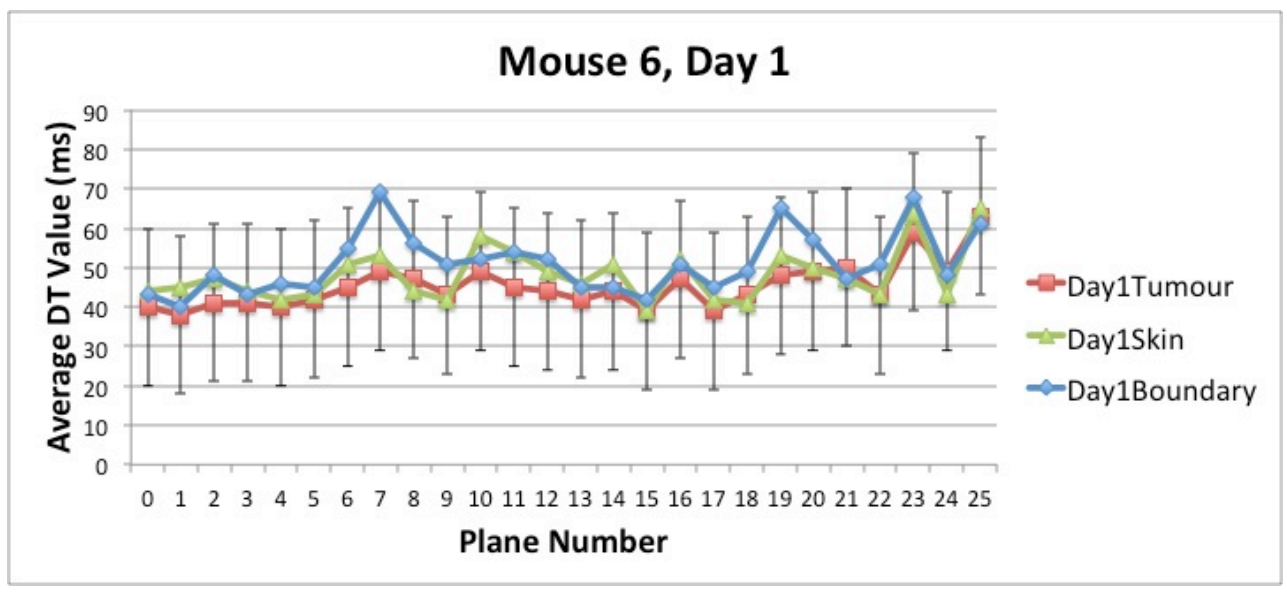

(b) 


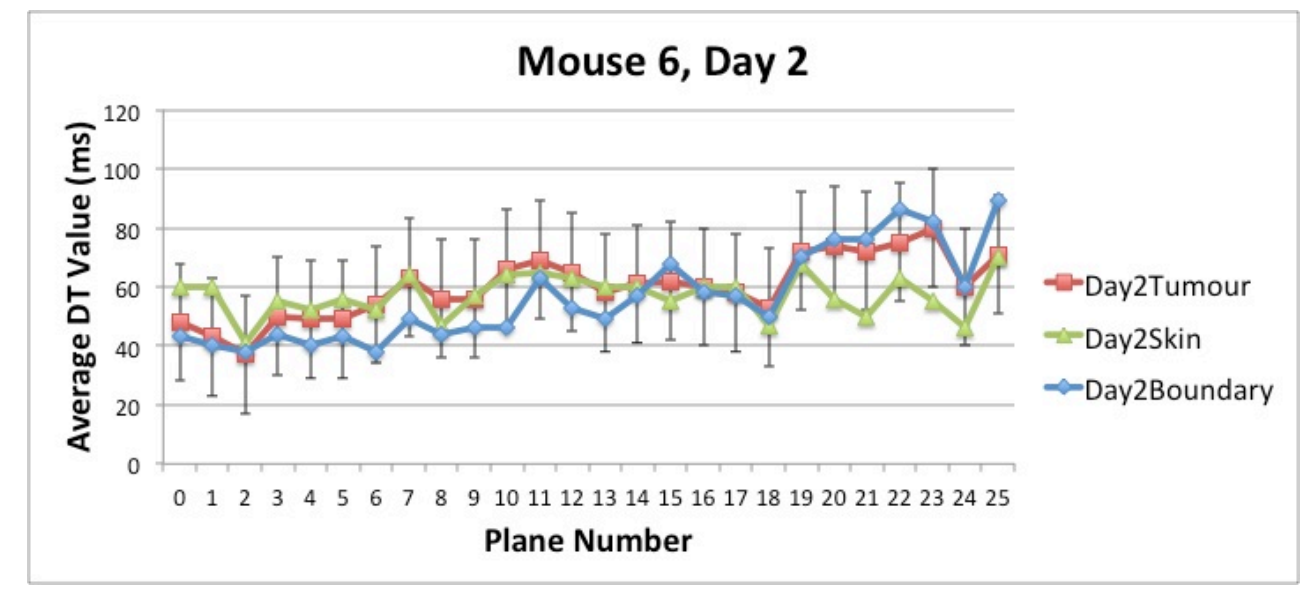

(c)

Figure 4-13: Average DT values of 3 ROIs ("tumour", "skin", and "boundary") across 26 imaging planes for Mouse 6 on (a) Day 0; (b) Day 1; and (c) Day 2. The error bars represent the standard deviation of the "tumour" data set. The standard deviation for the "skin" and "boundary" data sets were similar to the standard deviation for the "tumour" data set, but these error bars are not shown to prevent overlapping error bars.

The resulting average DT values were plotted in Figures 4-13a to 4-13c for Mouse 6 from Day 0 to Day 2. The average DT values showed fluctuations as the ROIs were mapped through the 26 imaging planes (625 $\mu \mathrm{m}$ total distance from plane 0 to 26 ), indicating that there were changes in the average DT through the different imaging planes. The average DT values for all three ROIs ranged from just under $40 \mathrm{~ms}$ to over $100 \mathrm{~ms}$ on Day 0, $70 \mathrm{~ms}$ on Day 1, and $90 \mathrm{~ms}$ on Day 2, as shown in Figures 4-13a to 413c. The overlapping of the three lines indicates the similarity of the average DT values for the three ROIs chosen. The data presented showed that the selection of ROIs of different sizes (e.g. when comparing the 9000-pixel "tumour" ROI and the 1500-pixel "boundary" ROI) for the same tumour sample did not show distinct differences between the "tumour" and "boundary" ROIs when mapping the average DT changes across the $625 \mu \mathrm{m}$ total distance. Another notable observation was the sudden increase or decrease in the DT when compared to the adjacent imaging planes. An example was plane 23 for 
Day 0 (Figure 4-13a) and Day 1 (Figure 4-13b), where a peak associated with an area of long DT was measured. This location could have been an area not as metabolically active, resulting in less motion and longer DT values.

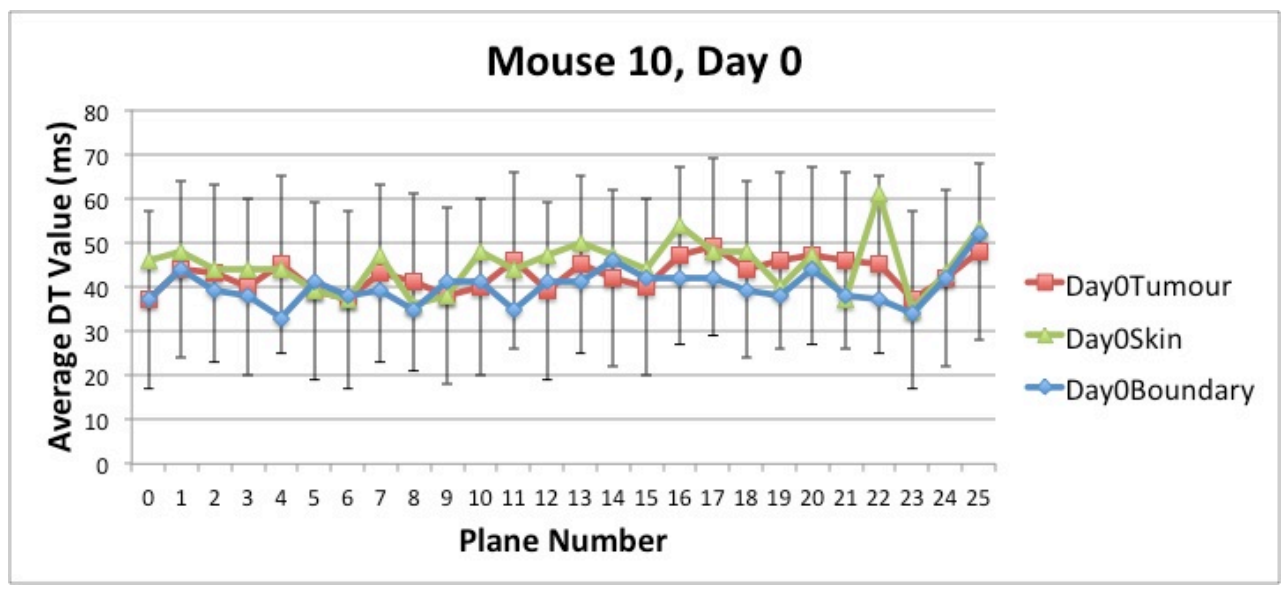

(a)

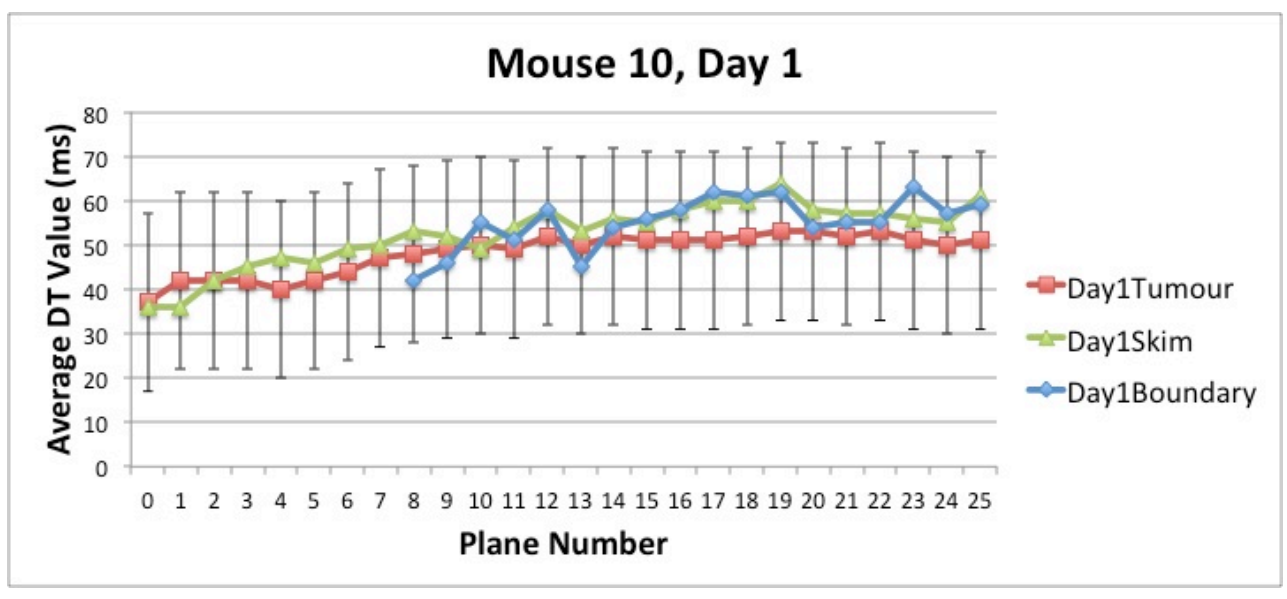

(b) 


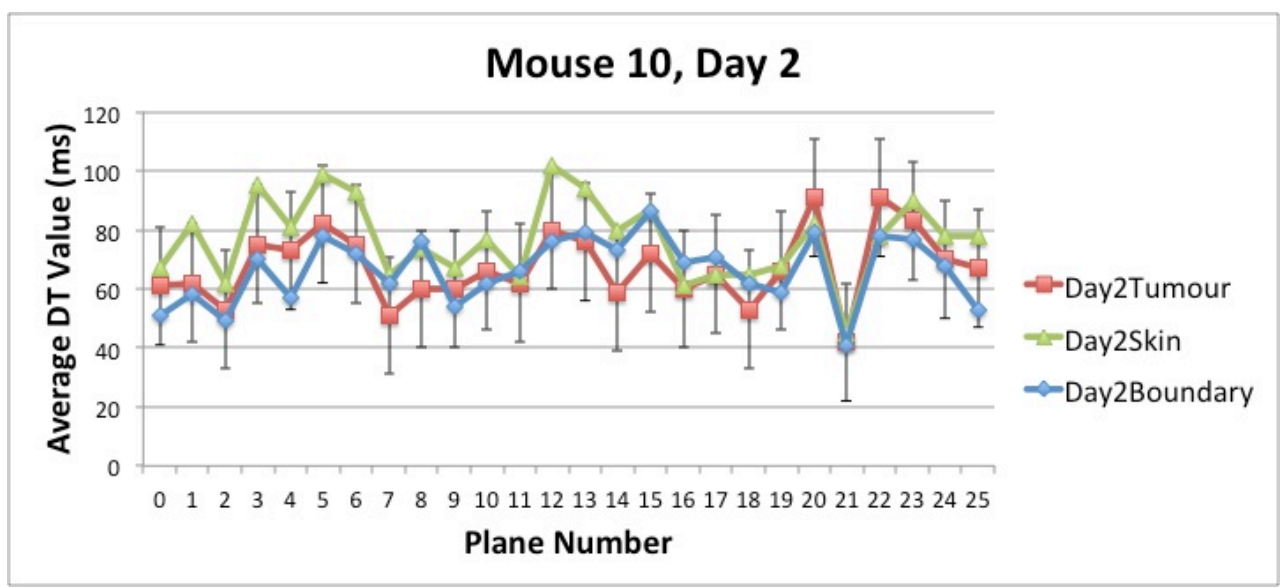

(c)

Figure 4-14: Average DT values of 3 ROIs ("tumour", "skin", and "boundary") across 26 imaging planes for Mouse 10 on (a) Day 0; (b) Day 1; and (c) Day 2. The error bars represent the standard deviation of the "tumour" data set. The standard deviation for the "skin" and "boundary" data sets were similar to the standard deviation for the "tumour" data set, but these error bars are not shown to prevent overlapping error bars.

Figures 4-14a to 4-14c show the average DT values for ROIs for Mouse 10 for Day 0 to Day 2. The data in these three graphs showed similar trends to the data of Mouse 6 in Figures 4-13a to 4-13c, with the range of average DT values from under 40 ms to over 60 ms for Day 0 and Day 1, and over 100 ms for Day 2. Note that in Figure 4$14 \mathrm{~b}$ for Mouse 10, Day 1, there was no data for the first 8 imaging planes of the boundary ROI. This was due to areas of short DT that were segmented out by the thresholding step, resulting in average DT values of zero. Possible reasons for these areas included a dense network of blood vessels or motion associated with significant tumour cell death. A sudden valley was also detected in plane 21 on Day 2, potentially indicating an area in the tumour having a stronger effect from the cisplatin drug causing motion and shorter DT values.

Similar results were obtained for all other cisplatin-treated mice in this study. The control mice also contained fluctuations in the line graphs. Because no cisplatin was 
administered to the control mice, the cause for the increase or decrease of the DT could have been from tumour heterogeneity itself. Areas of a dense network of blood vessels could also have been present in the ROIs chosen, thus yielding results where the DT averaging covered a smaller pixel count. A dense network of blood vessels can cause greater variability due to the smaller number of pixels that contribute to the averaging for the DT measurements. Also, due to the possibility that the imaging voxel $(8.6 \mu \mathrm{m}$ for each side) may contain a mixture of regions with cells and blood vessels, some contributions to the DT from blood vessels may not be segmented, thus resulting in contributions from signals from blood vessels being analyzed. This is possible throughout the whole tumour, but it is more probable in areas with a dense vascular network such as the tumour periphery and skin.

\subsubsection{Decorrelation Time Averages for Control and Treated Mice}

Table 4-4: The four categories (C1, C2, C3, C4) indicating the ROI selection with or without threshold. The ROI sizes are: large ( 100 by 200 pixels $=860 \mu \mathrm{m}$ by $1720 \mu \mathrm{m})$ and small ( 10 by 40 pixels $=86 \mu \mathrm{m}$ by $344 \mu \mathrm{m})$. Large ROI Small ROI

With Threshold

Without Threshold
Category 1 (C1) Category 3 (C3)
Category 2 (C2)

Category 4 (C4)

The average DT was measured to compare control versus treated mice, while varying the ROI size and the application of the thresholding to determine how this would affect the interpretation of the data and conclusions. Four different categories were established to compare the two factors of ROI size and thresholding (outlined in Table 44). For all four categories, control (Mouse 1 and 2) and cisplatin-treated (Mouse 5, 6, and 
10) mice were analyzed. The imaging planes for all four categories are shown in Figures 4-15a to 4-15d for Mouse 6 and Figures 4-16a to 4-16d for Mouse 10. These five mice were chosen since a well-defined tumour could be identified by the supporting H\&E histology.

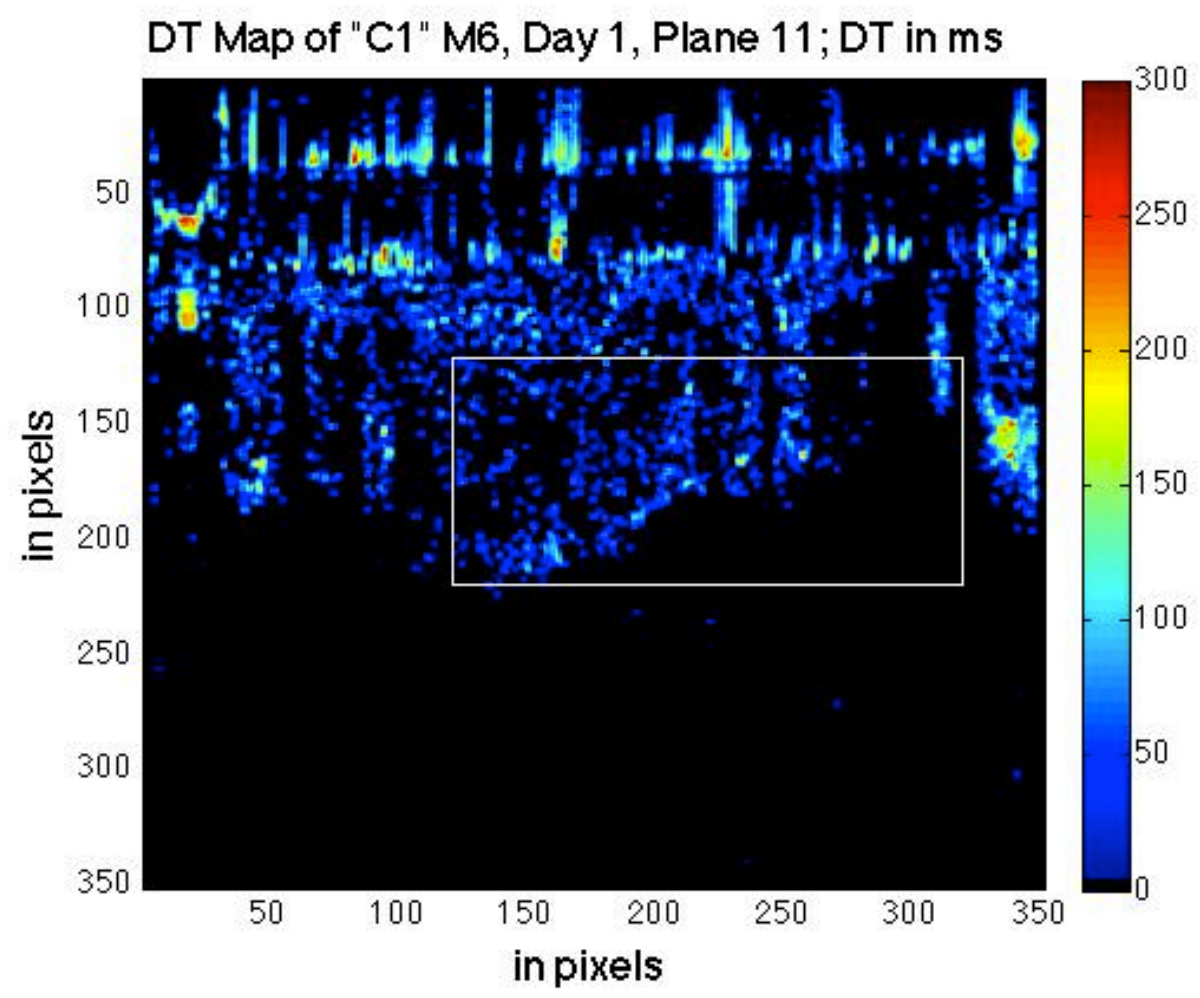

(a) 


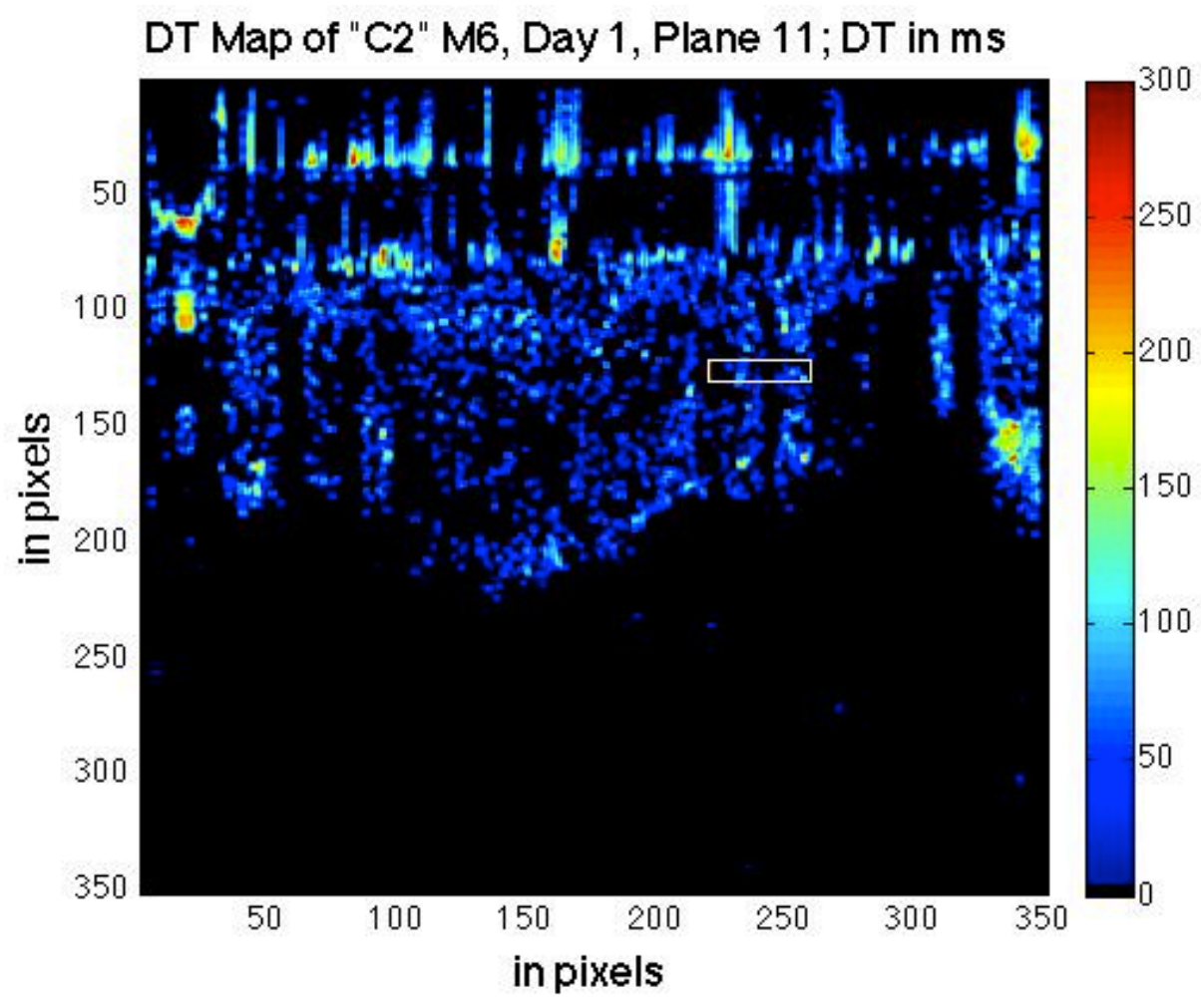

(b)

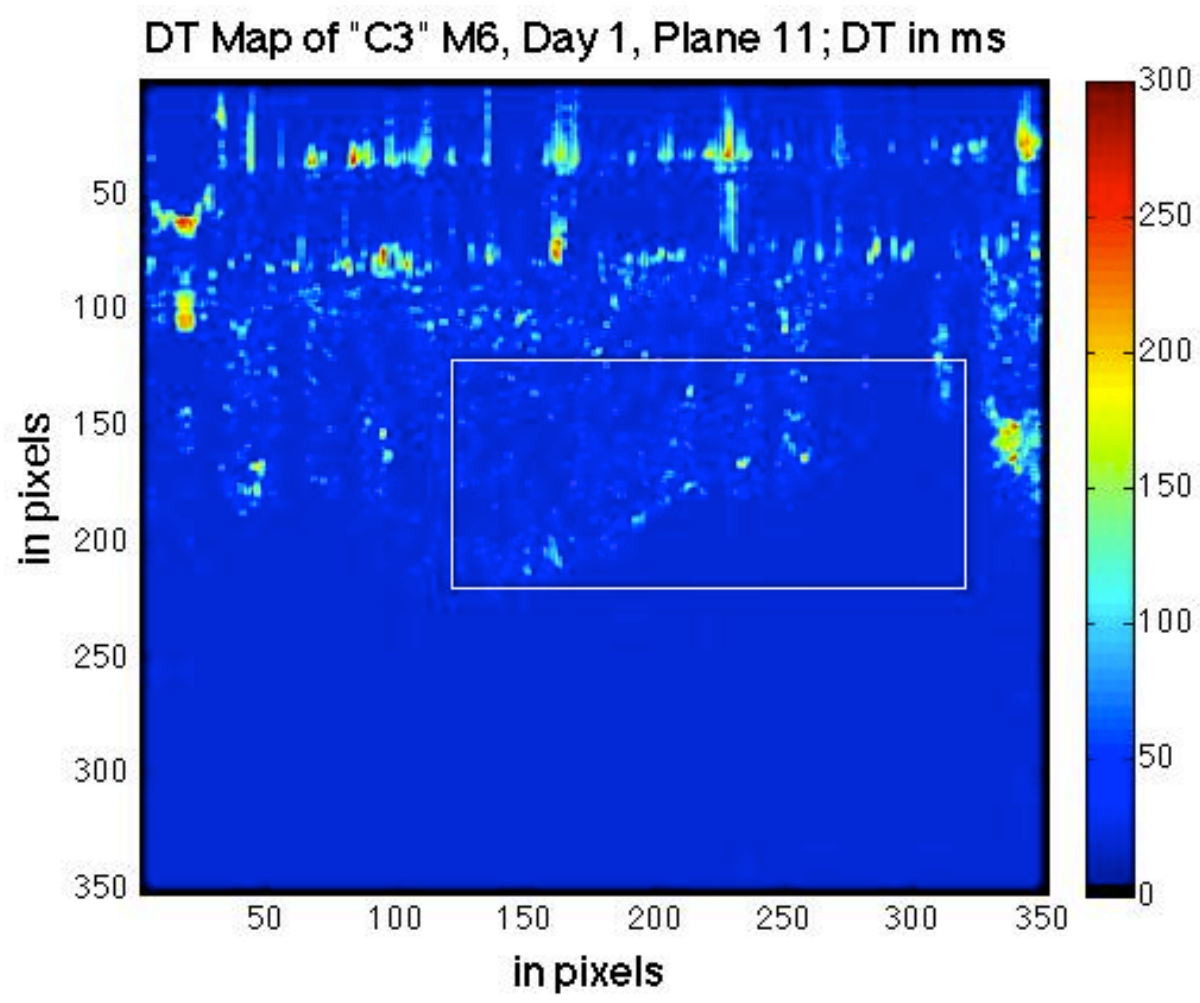

(c) 


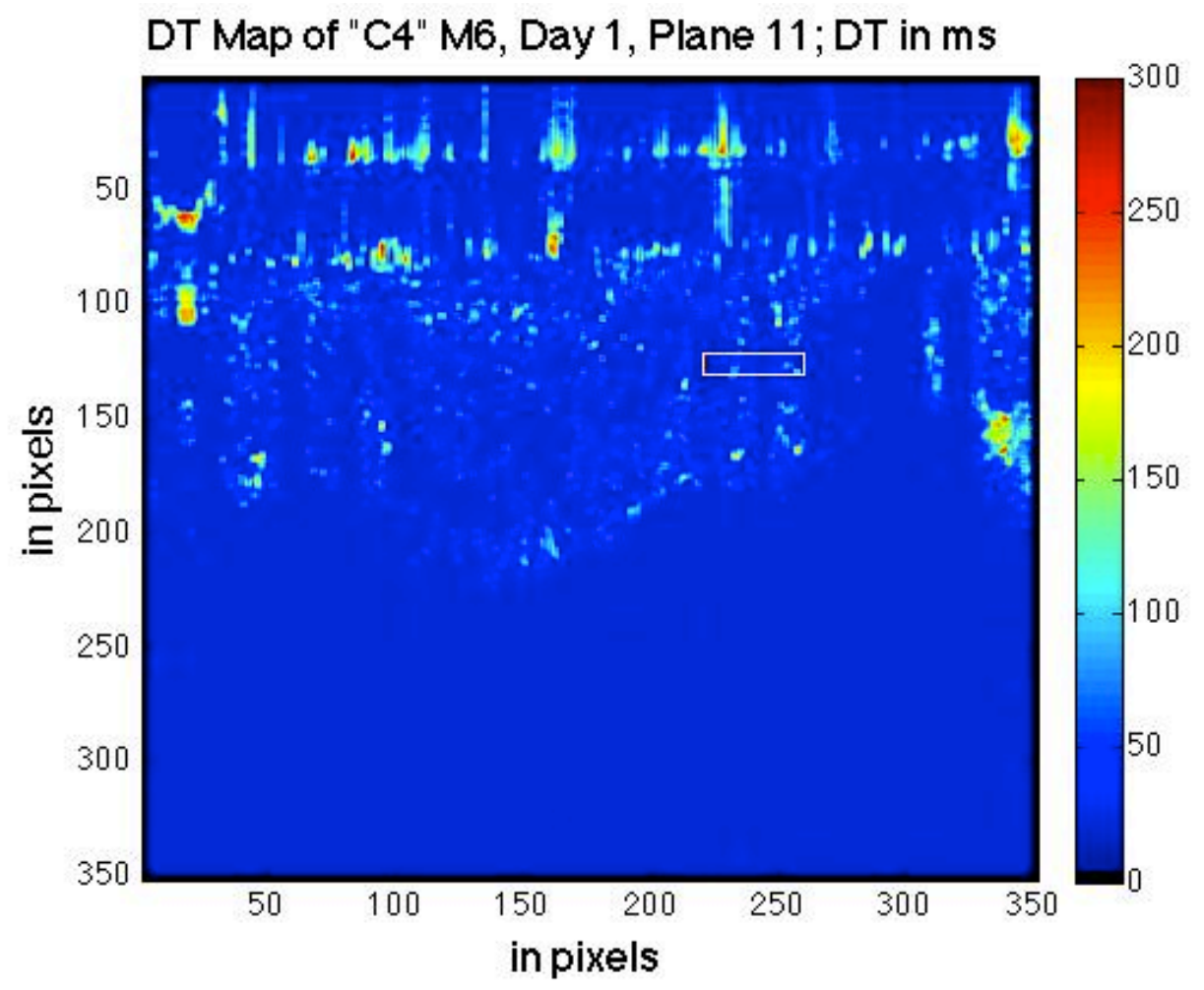

(d)

Figure 4-15: DT maps for Mouse 6, Plane 11, on Day 1 correspond to the four categories outlined in Table 4-4: (a) C1; (b) C2; (c) C3; and (d) C4. The image height and image width are both $3 \mathrm{~mm}$ for all images. 


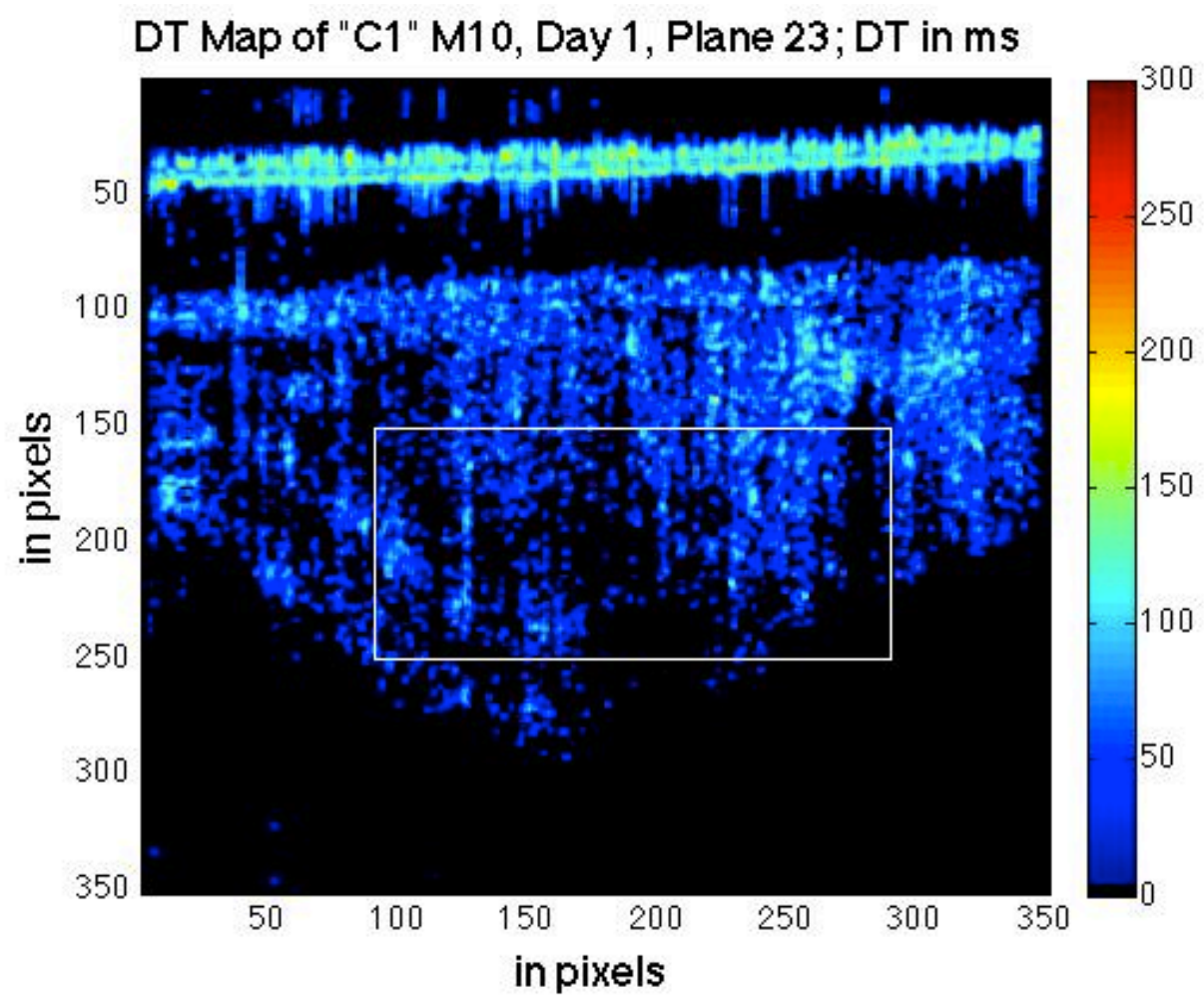

(a)

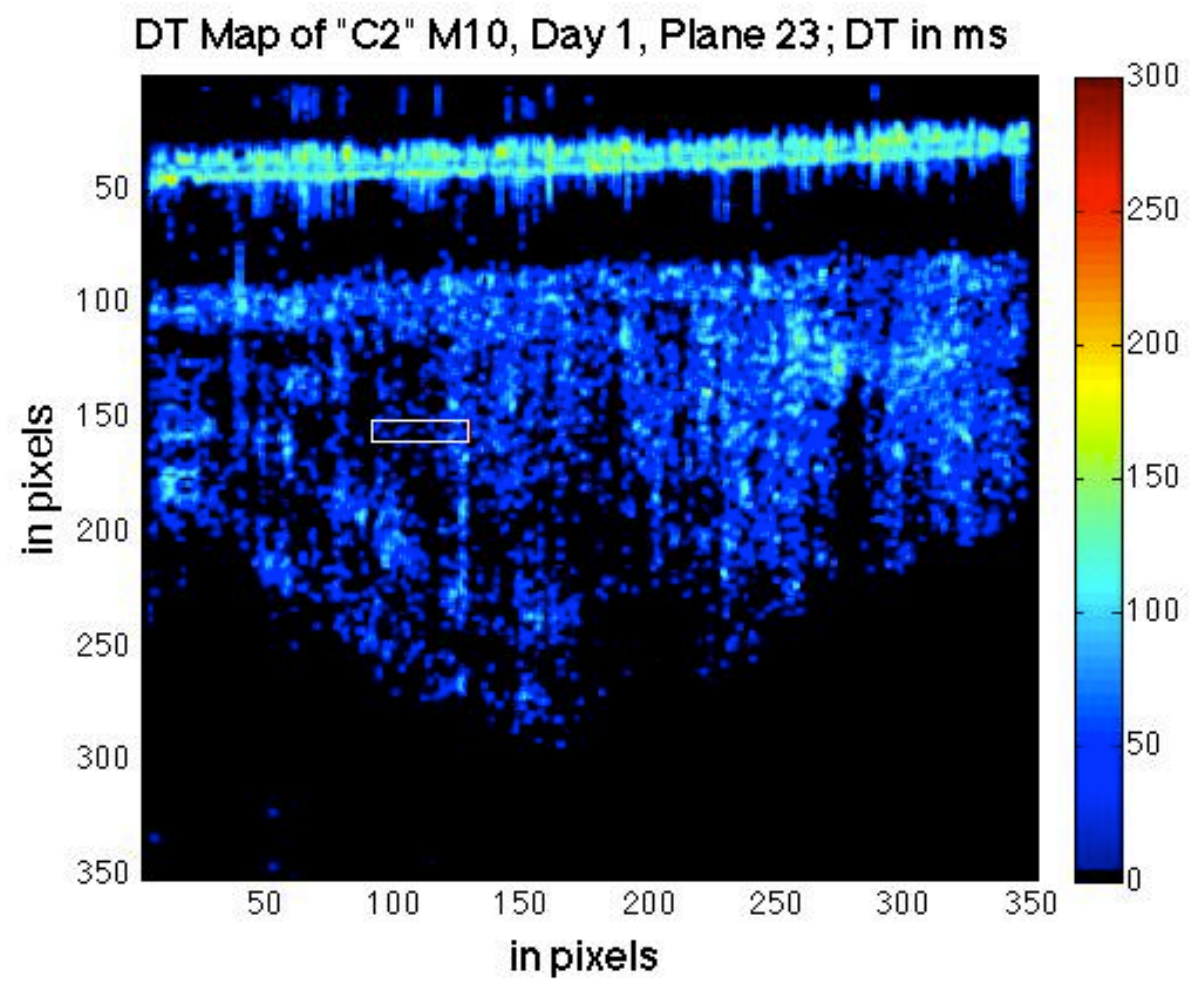

(b) 


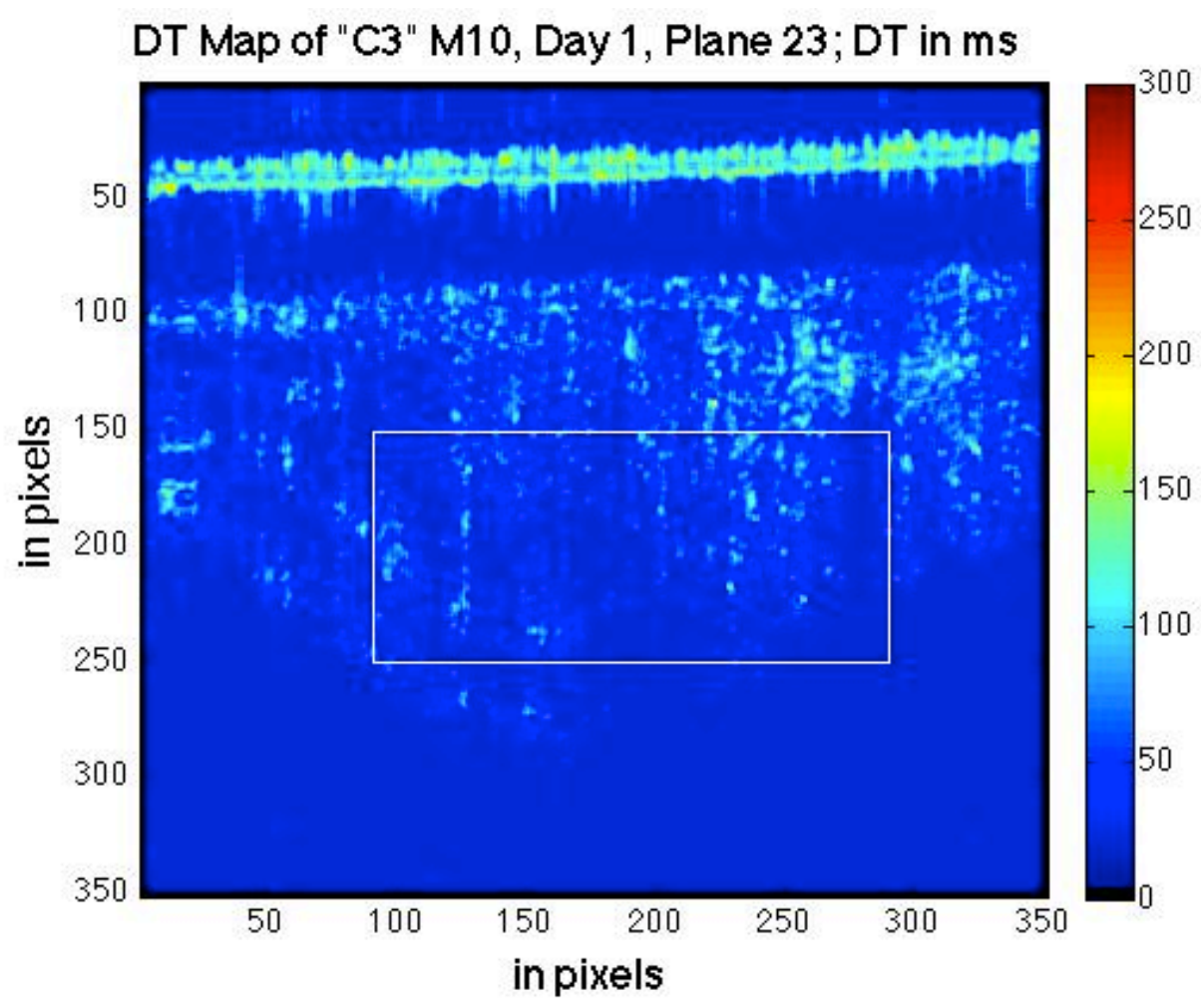

(c)

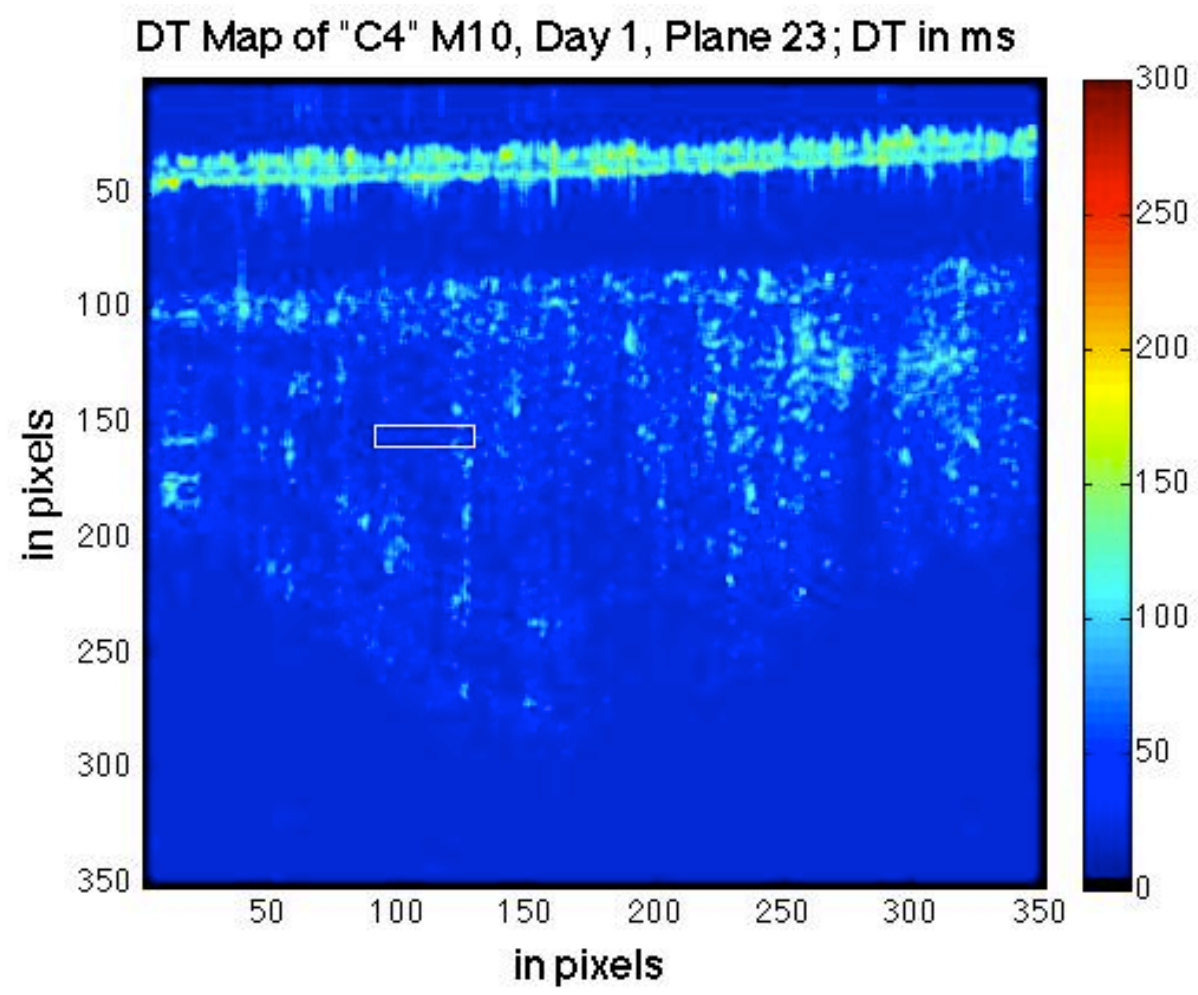

(d) 
Figure 4-16: DT maps for Mouse 10, Plane 23, on Day 1 correspond to the four categories outlined in Table 4-4: (a) C1; (b) C2; (c) C3; and (d) C4. The image height and image width are both $3 \mathrm{~mm}$ for all images.

For all five mice, the average DT was determined for the indicated ROI and plotted for all three days (where data is available for at least two days). The control and cisplatin-injected mice were plotted separately to view the differences in average DT over the treatment days. The error bars for each data point was determined by the standard deviation of the DT values for all the nonzero pixels in the selected ROI. The plots for all four categories are shown in Figures 4-17a to 4-17d.

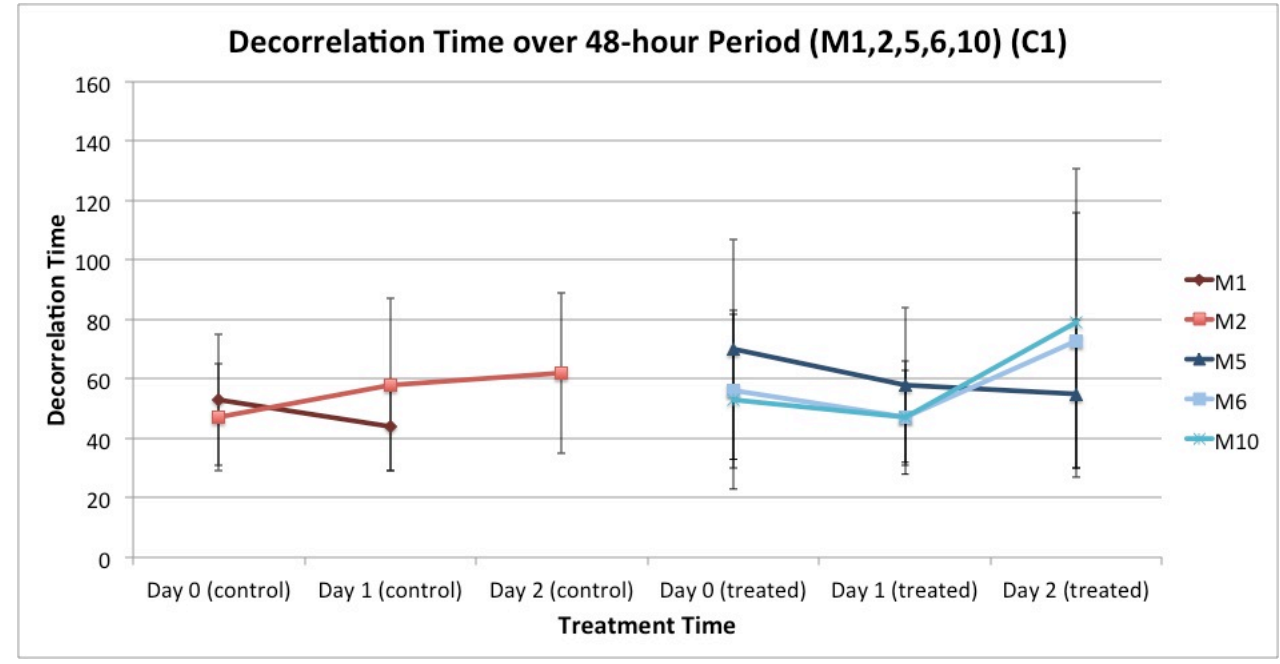

(a)

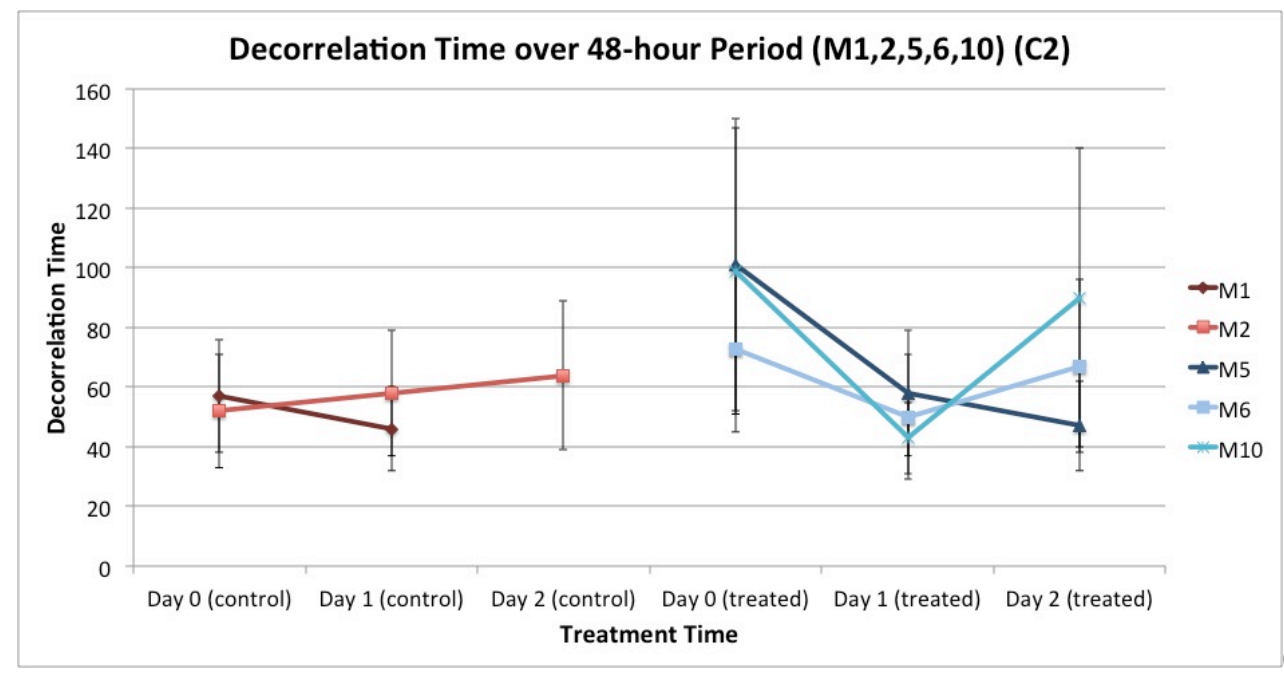

(b) 


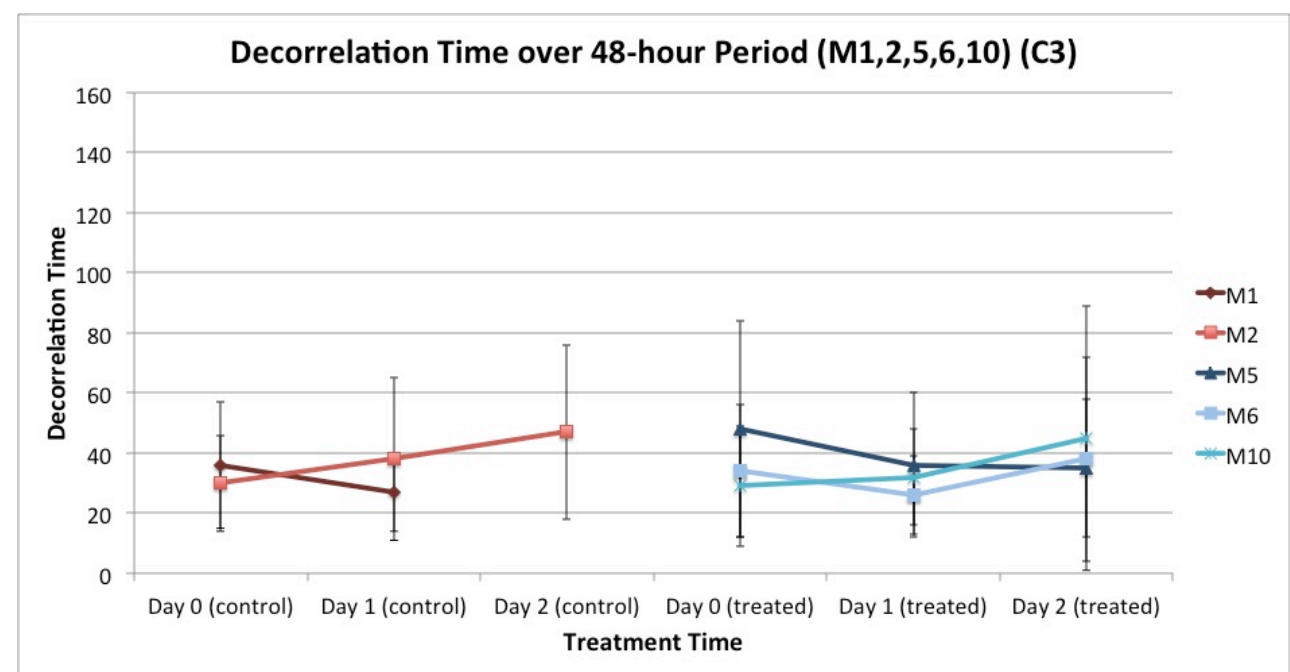

(c)

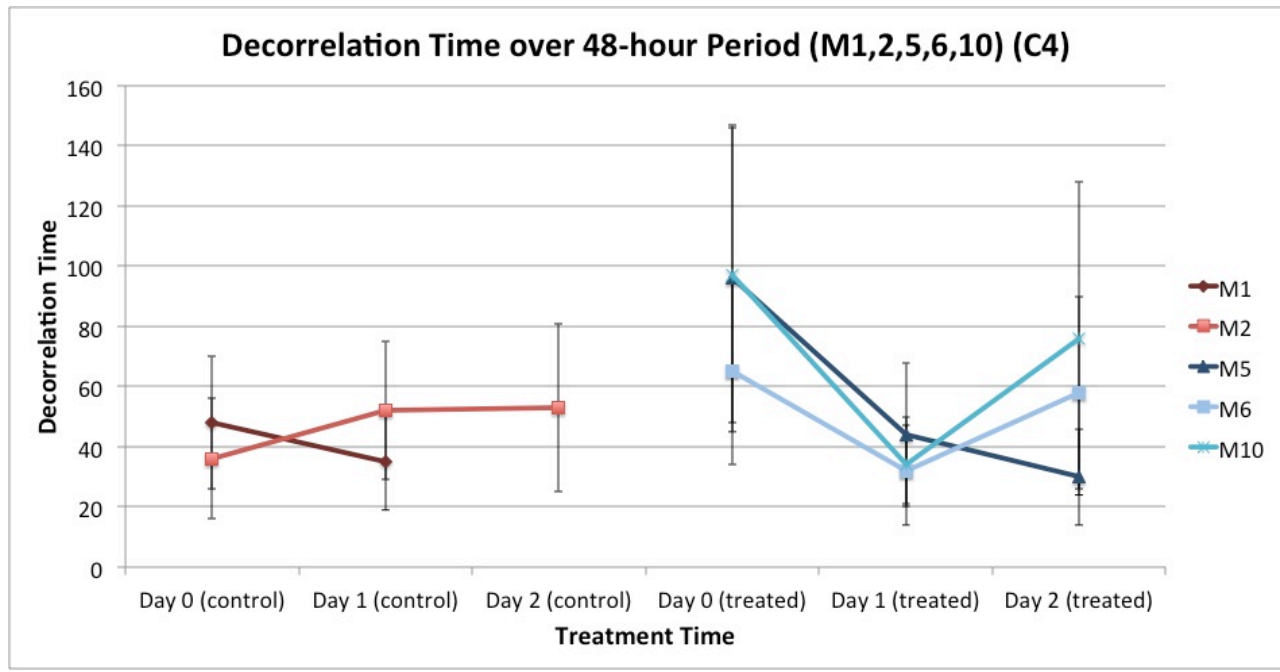

(d)

Figure 4-17: Average DT plots (in ms) for five mice over the 48-hour treatment period. Mouse 1 and 2 were control mice and are on the left columns labeled in red and brown. Mouse 5, 6, and 10 were cisplatin-injected mice and are on the right columns labeled in blue colours. Error bars refer to the standard deviation for the averaging of each ROI. The plots correspond to: (a) $\mathrm{C} 1$; (b) $\mathrm{C} 2$; (c) $\mathrm{C} 3$; and (d) $\mathrm{C} 4$.

The resulting data from the four plots of average DT over the 48 hours of treatment time for both control and cisplatin-treated mice showed trends of DT changes due to cisplatin effects.

Firstly, comparing categories $\mathrm{C} 1$ and $\mathrm{C} 2$, it was observed that the selection of the ROI size could potentially affect the average DT for cisplatin-treated mice only. The 
graphs for the control mice showed similar average DT throughout the 48 hours irrespective of the window size chosen. The drop in DT from Day 0 to Day 1 for the small ROI in cisplatin-treated mice is consistent with the in vitro cell death data [11]. The comparison of the $\mathrm{C} 3$ and $\mathrm{C} 4$ graphs showed the same graph shapes as comparing $\mathrm{C} 1$ and $\mathrm{C} 2$ graphs, even though there was no thresholding applied for categories $\mathrm{C} 3$ and $\mathrm{C} 4$.

The comparison between categories $\mathrm{C} 1$ and $\mathrm{C} 3$ was to investigate the effect of thresholding when analyzing a large ROI. The similarities in the graphs for the large ROI showed that the thresholding process did not significantly affect the changes in the average DT, but there was a vertical shift (of approximately $20 \mathrm{~ms}$ ) in the data when the thresholding was not applied. Because only nonzero values were used in the averaging within the ROI, the averaging in category $\mathrm{C} 1$ had a lower pixel count than category $\mathrm{C} 3$. The averaging in category C3 included the short DT values that were not segmented, resulting in shorter average DT values. This was also observed when comparing categories $\mathrm{C} 2$ and $\mathrm{C} 4$. The graph trends were the same and the vertical shift was also observed. The average DT values and pixel counts for the data in categories C1, C2, C3, and $\mathrm{C} 4$ are shown in Table D-3 in Appendix E.

The data presented in the graphs in Figures $4-17$ a to $4-17 d$ showed the effect of applying the threshold of $30 \mathrm{~ms}$ to segment out pixels of short DT. This data also more clearly illustrates the initial DT drop for Day 1 for cisplatin-treated mice one day after exposure.

Statistical analysis was performed on the data presented in Figure 4-17 to verify the results presented. Table 4-5 presents an excerpt of the results from the Student's t-test performed to compare the means of the average DT values recorded for the control and 
cisplatin mice. The control mean was found by averaging the average DT values for control mice \#1 and 2 . The cisplatin mean was found by averaging the average DT values for cisplatin mice \#5, 6 and 10. The assumptions for the Student's t-test were that the populations of the random errors had a normal (Gaussian) distribution, and there was no significant difference between the standard deviations of both population samples. Appendix E has the full summary chart.

Table 4-5: An excerpt of the Student's t-test to compare the means of the average DT values (in ms) recorded for the control and cisplatin mice in Figure 4-17. See Appendix E for the full summary chart.

\begin{tabular}{|cccc}
\hline \multicolumn{4}{c}{$\mathbf{C 2}(\mathbf{n}=\mathbf{4 0 0}$ for all mice); Student's t-test } \\
\hline & Day $\mathbf{0}$ & Day $\mathbf{1}$ & Day $\mathbf{2}$ \\
\hline Control mean & 54.5 & 52 & 64 \\
\hline Cisplatin mean & 86 & 46.5 & 78.5 \\
Control Std. Dev. & 19 & 17.85 & 25 \\
\hline Cisplatin Std. Dev. & 42.77 & 18.49 & 34.48 \\
\hline Pooled Std. Dev. & 36.61 & 18.28 & 34.48 \\
\hline t-exp & 0.9426 & 0.3296 & 0.3642 \\
\hline t-th & 3.182 & 3.182 & 4.303 \\
\hline Decision & Retain $\mathrm{H}_{0}$ & Retain $\mathrm{H}_{0}$ & Retain $\mathrm{H}_{0}$ \\
\hline
\end{tabular}

The null hypothesis $\left(\mathrm{H}_{0}\right)$ is the control and cisplatin means are the same. All of the experimental t-values (t-exp) were less than the theoretical t-values (t-th) at $95 \%$ confidence level, resulting in insufficient data to reject the null hypothesis. Therefore, the statistical analysis showed that the control DT mean and cisplatin DT mean were the same, and any differences would be random errors and not systemic errors.

\subsection{Histology Analysis}




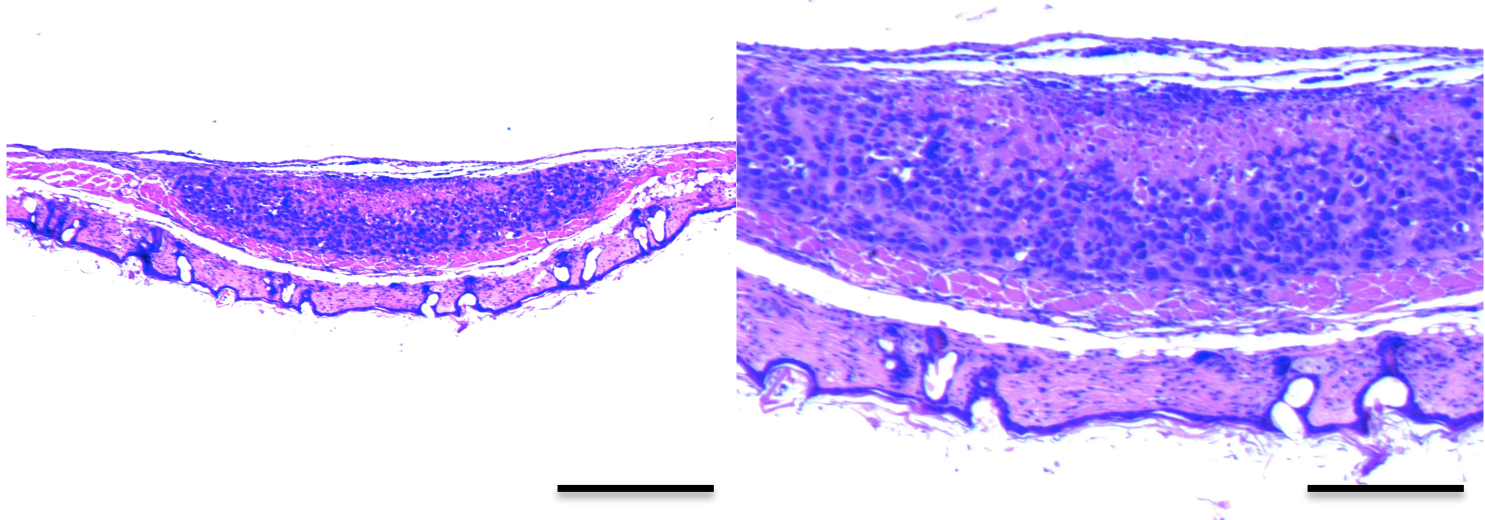

(a)

(b)

Figure 4-18: H\&E staining for Mouse 6 at microscope objective magnifications of (a) 4x $($ Scale bar $=500 \mu \mathrm{m})$ and $(\mathrm{b}) 10 x($ Scale bar $=200 \mu \mathrm{m})$.

Hematoxylin and eosin (H\&E) staining was performed by the Pathology Research Program at the University Health Network for all ten mice in this study to observe the morphology of the dorsal skin tumour tissue in the window chamber model. The sectioning presented tumour growth for mice \#1, 2, 3, 5, 6, and 10. Tumour regions were not found in the slides for mice \#4, 7, 8, and 9, and thus were not used in the DT analysis of control and cisplatin-injected mice. Figures 4-18 and 4-19 show the histology sections for Mouse 6 and 10, respectively, utilizing objective magnifications of 4x and 10x. Similar morphology was observed in the slides for mice \#1, 2, 3 and 5. 


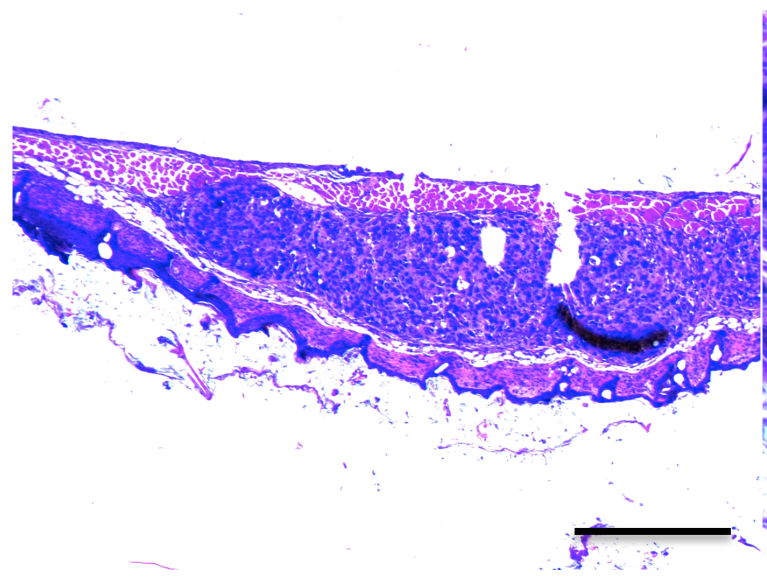

(a)

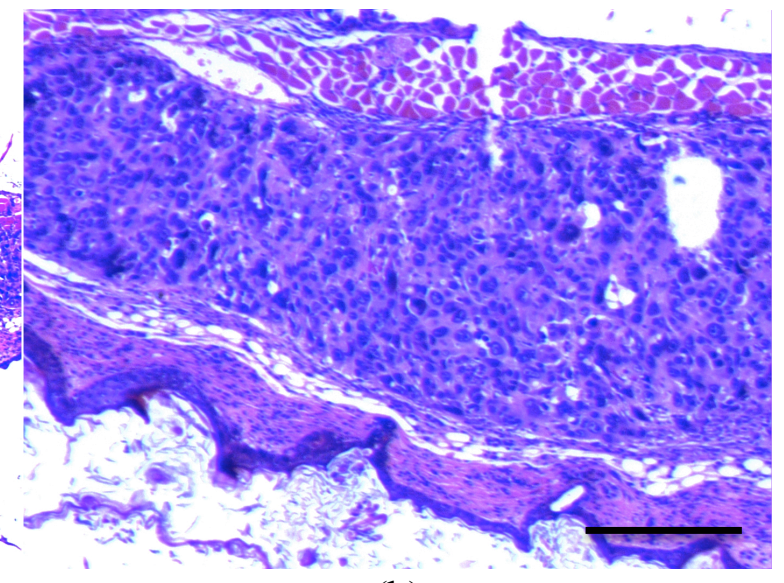

(b)

Figure 4-19: H\&E staining for Mouse 10 at microscope objective magnifications of (a) $4 \mathrm{x}($ Scale bar $=500 \mu \mathrm{m})$ and $(\mathrm{b}) 10 \mathrm{x}($ Scale bar $=200 \mu \mathrm{m})$. 


\section{Chapter 5: Discussion}

The results from the experiments demonstrated that DLS-OCT could be used to measure the decorrelation time in an in vivo mouse tumour model. The OCT b-mode images, decorrelation time maps, and the histology images acquired in this thesis provided an initial assessment for the type of information that is available in the analysis of tumour growth using motion as a source of contrast in OCT. A segmentation technique was developed to separate tissue regions from vessels regions within a ROI, based on the DT values. The decorrelation time analysis of the changes in average DT for control and cisplatin-treated mice across the different imaging planes also provided an insight into the variations within a tumour and how these values change longitudinally for monitoring tumour growth in an in vivo setting. The histology results provided another means to observe the location of the tumours in the dorsal skin tumour tissue. The data collected in this thesis illustrate the potential for in vivo research of observing fluctuations in scatterers using decorrelation time analysis to study tumour response. A discussion of the results from this thesis follows in this chapter and will provide the foundation for future work in Chapter 6.

\subsection{Flow Segmentation}

A threshold was placed for all DT maps to segment out any short DT contributions. The previous in vivo data had indicated motion due to blood flow as a cause for patches of short DT. Therefore, it was important in this thesis to provide the correct threshold to segment out pixels of short DT before completing any further DT 
analysis. The threshold found most effective for all mice in this study was at DT $=30 \mathrm{~ms}$. This threshold provided the most distinct visual qualitative change in the DT maps, while preserving the apparent structure of blood vessels. The threshold of $30 \mathrm{~ms}$ seemed to have removed a large portion of short DT pixels that we hypothesize were from blood vessels (Tables 4-2 and 4-3). The increase in the average DT value between the thresholds of 20 to $30 \mathrm{~ms}$ was also the greatest in comparison to 30 to $40 \mathrm{~ms}, 40$ to $50 \mathrm{~ms}$, and 50 to $60 \mathrm{~ms}$. The removal of DT contributions at vessel locations allowed for greater accuracy in monitoring the response of tumour cells to treatment via the amount of motion.

The longest and shortest DT values measured were 617 and $20 \mathrm{~ms}$, respectively, as indicated in Section 4.2 and Figure 4-6b. DT measurements have a lower limit of 20 ms in our experimental system due to the Nyquist frequency. The images in this experiment were scanned at a frame rate of 100 frames per second, or 1 frame per 10 milliseconds, resulting in $10 \mathrm{~ms}$ being the shortest possible DT measured. However, due to the Nyquist frequency being half of the sampling frequency of $100 \mathrm{~Hz}$, the actual shortest recorded DT was $20 \mathrm{~ms}$. The longest recorded DT value was $617 \mathrm{~ms}$, which was located at a pixel corresponding to the glass coverslip of the window chamber. This result was reasonable, as a fixed piece of glass should have minimal motion recorded. However, it does not guarantee $617 \mathrm{~ms}$ is the upper limit of DT as there are many different causes of motion, such as breathing motions and noise inherent to the imaging system. These factors can change the longest DT value recorded, even for something rigid as glass.

\subsection{Decorrelation Time Analysis}


The DT analysis of different ROIs across adjacent imaging planes yielded the following result: for three ROIs of different sizes and locations, the average DT values across the $625 \mu \mathrm{m}$ total distance were similar. Figures $4-13$ and $4-14$ showed the overlapping of the average DT values for all three ROIs ("tumour", "skin", "boundary") across the 26 imaging planes of Mouse 6 and 10. Future work includes analyzing more "tumour", "skin", and "boundary" ROIs to verify the results obtained in this thesis. Another improvement would be to analyze more than 26 imaging planes in order to map a larger distance of average DT to generate a greater scope of the differences in tumour growth along its diameter.

The DT of different ROIs for control and cisplatin-treated mice was also analyzed to study tumour treatment response. In this thesis, ten mice were observed for the changes in average DT over treatment time of 48 hours. For this specific test, the data for five mice were used to illustrate how the average DT changed over treatment time, varying the ROI size as well as the use of the threshold of $30 \mathrm{~ms}$. The comparison of the four categories of data showed that the use of the threshold of $30 \mathrm{~ms}$ allowed for the removal of short DT contributions (most likely from vessels), and a vertical shift in the DT graphs was observed because of the change in the pixel values and count when excluding pixels with DT shorter than $30 \mathrm{~ms}$. The shape of the DT graphs did not change when applying the threshold, thus showing that although the threshold did help in providing more representative data related to cellular and extracellular structures, the threshold did not significantly alter the DT contributions from the tissue.

The differences in control and cisplatin-treated mice were also observed in this final assessment. The shapes of the graphs for the control mice for all four categories 
were the same, indicating no differences in average DT for ROI size. The average DT values for the control mice were similar throughout the 48 hours between the first and last imaging sessions. Small changes in the DT values can be attributed to metabolic changes of the tumour as it grows. In contrast, the treated tumours showed a difference in the DT values as a function of time. The analysis revealed a drop in the DT from Day 0 to Day 1 for cisplatin-treated mice, which suggests that the tumour is responding to the cisplatin treatment. It is known that apoptotic cells (in this case, due to the cisplatin exposure) undergo cell shrinkage and nuclear fragmentation resulting in increased cellular and intracellular motion. This was a key observation as it corresponded to the previous in vitro results of a drop in DT for Day 1. Note that this drop in DT was observed more significantly for the small ROI. Therefore, the specific ROI size chosen could affect the DT results obtained, due to the heterogeneity of the in vivo tumour tissue.

The DT analysis of the changes in average DT for different ROIs across different imaging planes yielded results of the similarity of the mix of DT values throughout the same tumour region. The DT analysis of the changes in average DT for control and cisplatin-treated mice provided initial results for monitoring tumour growth in an in vivo setting, such as observing the increases and decreases of DT at certain treatment times for specific ROI size, as well as supporting the use of the threshold of $30 \mathrm{~ms}$ for segmenting out the DT contributions from blood vessels. This work demonstrated that it was feasible to use DLS-OCT to analyze mouse tumours in vivo and obtain results that are similar to previous in vitro and in vivo studies. It was also shown that DT contributions of vessels could be segmented apart from tissue regions. Further studies and enhancements to this 
work are required to draw definite conclusions of the changes in average DT, and will be discussed in the next chapter.

\subsection{Image Acquisition}

The SS-OCT system used for the DLS-OCT imaging allowed for fast OCT bmode imaging of the tumour tissue. Because of its $36 \mathrm{kHz}$ scan rate, the duration for obtaining 1000 frames for each imaging plane of tumour data was 10 seconds. Therefore, the total time required for imaging one mouse tumour (26 planes) was under five minutes. The drawbacks to this technique were the storage size for the OCT data within each imaging plane and the sensitivity of the imaging system to breathing movements. The file for each OCT plane of 1000 frames amounted to 968 MB. The total amount of storage space required for the ten mice study was $605 \mathrm{~GB}$. This was significant because an ideal design of the imaging layout was to scan through the whole 1-2 mm diameter of the tumour. Even though only $625 \mu \mathrm{m}$ of tissue was scanned, it required $605 \mathrm{~GB}$ of storage space. Therefore, storage space is an issue that needs to be improved for collecting enough data to map out the whole tumour. The breathing movements of the mouse also affected the data collected with the SS-OCT system. Slight shifts of the tumour tissue were detected in the data; hence, the resulting DT data would have measured not only the tumour motion, but also the breathing motions of the mice. 


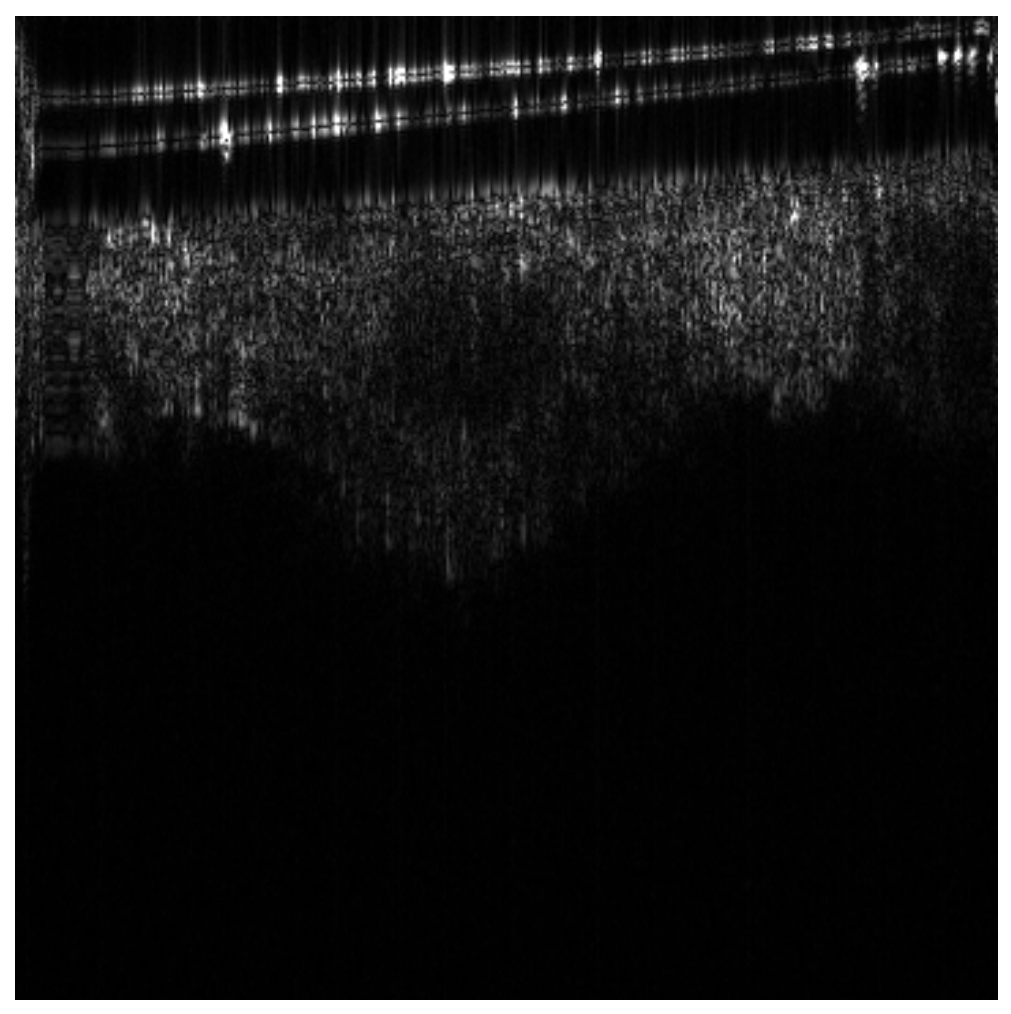

Figure 5-1: Mirroring artifact observed in this cross-sectional b-mode image of the tumour for plane 22 of Mouse 5 on Day 1. The image width and image height are both 3 $\mathrm{mm}$.

Using a fixed stand that holds the tumour tissue (window chamber) in place, or imaging the mouse when the anesthesia effect is at its peak can minimize the breathing motions detected in the OCT images. In addition, a mirroring effect was observed for the OCT bmode images obtained for Mouse 1 and 5. An example of this is shown in Figure 5-1. This effect may be attributed to a slight misalignment with the returning signal at the circulator. This is a critical issue to the data collection as any artifacts from the OCT bmode images will be translated to the DT maps. It is of utmost important in the future to check all optical components used in the imaging system before performing the imaging experiment. Lastly, there was noise in the measurements detected that affected the resulting OCT and DT images. Shot noise from the detector, instability of any 
mechanical parts of the imaging system, and aberrations in any of the fibres in the optical path will result in noise in all of the OCT images. An ideal estimate of the amount of noise in the OCT and DT images produced is to observe the recorded pixel intensities at a location of something rigid. A suggested location would be the largest recorded DT value of $617 \mathrm{~ms}$ corresponding to the glass coverslip of the window chamber. The intensities recorded at the glass coverslip would provide an ideal estimate of motions detected from the noise of the imaging system as the rigid and fixed glass piece would be assumed to have minimal motion.

The histology images acquired included H\&E staining to observe the morphology of the cells and to visually differentiate between tumour and non-tumour cells. A suggested improvement to the image acquisition of the H\&E slides would be to section the tumour tissue that matches with the specific imaging plane obtained in the OCT bmode data. This difficulty in this step is to accurately locate the same locations as the OCT images, and then subsequently provide precise micron-thick sections for the H\&E slides at those locations. The benefit of having spatially co-registered histology image acquisition is that the structural changes in the tumour as a function of time (tumour growth) or treatment time (tumour response) can be correlated to the changes in cellular structure as assessed by the histology. This may provide insight into the structural changes in tissue that cause the measured changes in the DT.

\subsection{Histology Analysis}

The H\&E staining showed the clear presence of tumours for mice \#1, 2, 3, 5, 6, and 10. Although the tumour was evident to the naked eye when performing the mice 
imaging due to a small bump where the tumour cells were implanted into the dorsal skin of the mice, this step of histology analysis was important to ensure that the tumours were observed in the DT maps. The H\&E slides provided distinction between the morphology of tumour and non-tumour cells. The tumour shape in the histology also allowed for the comparison of the gross tumour shape observed in the DT maps, but the co-registration of the specific location of the histology slide with the exact OCT b-mode imaging plane could not be done. A significant improvement to this experiment would be to have careful histology sectioning to match the OCT b-mode images recorded.

Another addition to the histology data for this experiment would be to perform additional staining besides H\&E staining. Terminal deoxynucleotidyl transferase dUTP nick end labeling (TUNEL) is a method that can be applied to detect DNA fragmentation by labeling the terminal end of nucleic acids. This method would provide additional data to support the tumour analysis because TUNEL detects DNA fragmentation that results from apoptotic signaling cascades. Therefore, TUNEL assays can aid the location of cell apoptosis and verify if those locations are where short DT is observed. In addition to TUNEL, platelet endothelial cell adhesion molecule or cluster of differentiation 31 (CD31) staining can also be performed to mark endothelial cells. This staining would allow for the location of blood vessels due to the endothelial lining in the vessels. This would extremely help the assessment of the threshold algorithm as it can verify if the thresholding is performed at the intended locations in the tumour region. 


\section{Chapter 6: Conclusions and Future Work}

Dynamic light scattering with optical coherence tomography was shown to be a feasible approach for performing speckle decorrelation time analysis in an in vivo mouse tumour model. The segmentation of decorrelation time contributions for suspected vessel regions was also performed successfully.

\subsection{Realization of Thesis Objectives}

There were two key objectives this thesis intended to achieve. The first objective was to perform and measure speckle decorrelation time analysis for control and cisplatininjected mice over a 48-hour treatment period to observe tumour growth and treatment response kinetics, and then to compare this with histological analysis. Because this thesis involved ten mice in the study, the hope was to demonstrate the feasibility of the DLSOCT measurements, implement a technique to remove vascular contributions to the tumour tissue DT data, as well as investigate how the DT of tumour tissue changes with treatment time. A technique was developed to segment contributions from blood vessels within tumours based on the DT data. The data presented in this thesis showed that DLSOCT could be used to measure DT for control and cisplatin-treated mice. This thesis, however, did not present any conclusive average DT trends, but rather, data on the DT changes were obtained with suspected trends that warrant further experiments to support the initial data in order to arrive at conclusions for the changes in average DT. One reason may be the weak response of the tumour to the treatment, as suggested by the analysis of the histology data. 
The second objective for this thesis was to determine a threshold value to perform the segmentation of the DT contributions from the microvasculature from the tumour tissue. The previous in vivo study measured DT that also included contributions from blood vessels from within a rectangular ROI. In this work, unwanted DT contributions from regions of blood flow were subtracted by finding a valid threshold value in the postprocessing step of data analysis. The DT maps for all ten mice were observed and different threshold values were utilized for each DT map to determine the most acceptable threshold value to use. The qualitative results from visualizing the different DT maps with the respective threshold values, and the quantitative results from analyzing the changes in the average DT based on the threshold values both revealed the threshold value of DT $=30 \mathrm{~ms}$ was a suitable choice for the thresholding.

\subsection{Future Work}

The results from this ten-mice study of DLS-OCT to measure the decorrelation time in an in vivo mouse tumour model was a first step for a larger study to fully analyze and monitor tumour growth in vivo. This experiment can be improved by: a) developing better 3D vessel mapping to unambiguously identify vessel locations, b) imaging a greater portion of the tumour volume (more tumour imaging planes), c) varying the tumour sizes beyond 1-2 $\mathrm{mm}$ in diameter to observe different tumour stages and how they respond to the treatment, d) ensuring that the treatment protocol induces strong tumour response, e) increasing the number of mice to be included in the study, and f) use more specific histological stains to identify the viability of tumour and endothelial cells. A repetition of this experiment with the aforementioned enhancements would likely provide 
more conclusive results about the potential of DT maps for tumour monitoring and the possibility of this DLS-OCT technique for in vivo treatment monitoring in general.

More accurate spatial vessel mapping can greatly enhance the ability to map out a region of vessels using DLS-OCT and speckle variance analysis. Vessel mapping involves the compilation of multiple two-dimensional OCT imaging planes to form a three-dimensional OCT volume structure, and the subsequent application of a specialized speckle variance algorithm to locate and track blood flow within the tumour volume. Having this vessel data, in addition to the algorithm used for forming DT maps, would allow the user to have a 3D OCT volume structure, its respective 3D speckle variance volume, as well as the accompanying 3D DT volume. The benefits of having all three types of data for the same tumour tissue would allow researchers to expand on this work and study the different types of blood flow and tumour growth. This methodology can theoretically be implemented for all types of tumours on any tissue structure to observe the tumour growth pattern and monitor any tumour treatment effects. Importantly, spatial and temporal correlations can be made of blood vessel function (flow) and tumour cell response adjacent to these vessels. Suitable data storage must be designed to support the expansive data for this type of research extension.

In this thesis, the OCT imaging was performed on 26 two-dimensional imaging planes of the size $3 \mathrm{~mm}$ in lateral distance and depth. This was effective in measuring a small portion of tumours that were 1-2 $\mathrm{mm}$ in diameter, but there are often cases where the desired target is larger than this. This is a small number of planes in order to form the entire tumour volume. Therefore, the increase in the imaging field of view as well as the 
number of imaging planes used to form a suitable 3D volume would involve longer data acquisition times and storage space to accommodate for the extra data acquired.

To better sample the different tumour stages and kinetics for the specific cell line, the tumours can be grown to larger sizes. The tumours analyzed in this thesis were all grown for two weeks, which resulted in tumours that were $1-2 \mathrm{~mm}$ in diameter. In order to extend to tumour phenotype and study how these tumours respond to treatment, the user can choose to vary the duration of tumour growth before the imaging experiments in order to observe the different tumour stages and how they respond to the treatment.

The treatment duration can also be adjusted in future experiments in order to record more data at different time points during the experiment. For example, the researcher may choose to collect data every 6 hours within the first 48 hours after treatment, as well as collect data after the first 48 hours. The drawback is similarly described above, involving the large amounts of storage space required. Also, if the mice are imaged more frequently, the level of anesthesia must be adequately adjusted to prevent mice mortality from the repeated stress from multiple cycles of anesthesia and imaging.

The ten-mice study was an improvement to the initial three-mice feasibility test, and having ten mice did provide more data to analyze to better establish trends of average DT changes. However, in order to provide more conclusive results, a larger scale experiment with more mice is required to observe the changes in DT over the 48-hour time period.

The histological staining can also be improved by having histological sectioning of the tumour tissue co-registered to match the OCT b-mode planes obtained in the 
imaging experiment. This will allow the user to have greater confidence in the correlation between the changes in the DT maps and how these relate to the biological changes suggested in the histology slides (e.g. tumour/endothelial cell death, fibrosis, etc.). Specific stains that can be used are stains to locate areas of DNA fragmentation and cell apoptosis (TUNEL staining) and the presence of blood vessels (CD31 staining).

Technological improvements in the imaging technique can be applied to further enhance the quality of the images acquired and the SNR of the DT measurements. Currently, the DLS-OCT imaging methodology involved the use of a SS-OCT imaging system. Within the field of OCT, there are always improvements that can be made to increase the output power and, therefore, the SNR. Faster OCT imaging instruments will help improve the DT measurements and expand the lower bound of the DT measurements. The continued advancement in OCT systems development will provide the optical means to improve on the data collected in DLS-OCT imaging.

Once the protocol for tumour monitoring is improved and the value of the measurement is rigorously established, the technology can be expanded to study human cancer treatment response to establish the consistency and reliability of the method clinically. A reliable method to monitor tumour response to treatment would have an immediate impact on healthcare: such an approach could be used to switch treatments if the tumour does not respond, saving the healthcare system the cost associated with failed treatments and the patient side-effects of ineffective treatments. 


\section{Appendix A: Excerpt of the Animal Protocol for the Mice Experiment}

AUP 1280.8

The following are sections pertaining only for this thesis.

\section{Protocol Descriptions/Design}

We have recently developed and demonstrated a new functional extension of optical coherence tomography capable of detecting cell death in vitro. Based on these results we are now ready to test this important imaging technique in vivo. The current protocol uses photodynamic therapy to induce tumour cell kill. However, PDT also causes a significant amount of vascular damage, which may cause difficulties for our new imaging technique. Thus we are proposing to use a $\mathrm{FaDu}$ (Head and neck, responsive to cisplatin treatment) cell line in conjunction with a single dose of cisplatin to evaluate the efficacy of our new functional imaging technique.

The dorsal skin fold window chamber will be installed in NCRNu (nu/nu animals), providing a free optical path for imaging and treatment. All tumours will be implanted into the fascia of the subcutaneous layer on the skin located opposite the window. The animals will be placed under general anaesthetic (isoflurane) and kept warm on a heated water blanket. To induce the tumours, 100,000 FaDu cells in $100 \mu 1$ of phosphate buffered saline will be injected between the fascia and the subcutaneous tissue using a $27 \mathrm{G}$ needle. Animals will be allowed to recover. They will then be assessed at 1, 6 and 24 $\mathrm{h}$ and then on a daily basis to ensure that no adverse effects arise. 
Cisplatin treatments will be started when the tumours reach 2-3 mm diameter. Once the tumours have reached this size they will be imaged the 24 hours prior to treatment using SV-OCT and our new functional imaging technique to assess the vascular and cellular state of the tumour. All OCT imaging will be done under our standard protocols using a mixture of xylazine/ketamine $(10 / 80 \mathrm{mg} / \mathrm{kg}) .24$ hours later the cisplatin will be administered via tail vein injection at a dosage of $100 \mathrm{mg} / \mathrm{m}^{2}$ once again under xylazine/ketamine anesthesia. The mice will then be imaged at one of the following time points after drug administration: $12 \mathrm{~h}, 24 \mathrm{~h}$, and $48 \mathrm{~h}$. A subgroup of mice $(\mathrm{n}=5)$ will be sacrificed at each time point and tumours resected for histochemical analysis and comparison to imaging results. Additionally there will also be a control group of untreated tumour bearing mice that will be sacrificed at the 24 -hour time point. Thus, the animal requirements for this amendment are,

Group 1 - Tumour Untreated Control - sacrificed @ 24 hour time point (N=5)

Group 2 - Tumour Treated - sacrificed @ 12 hour time point (N=5)

Group 3 - Tumour Treated - sacrificed @ 24 hour time point (N=5)

Group 4 - Tumour Treated - sacrificed @ 48 hour time point (N=5)

Total $\mathrm{N}=20$ 


\section{Appendix B: Images of the Mice Experiment}

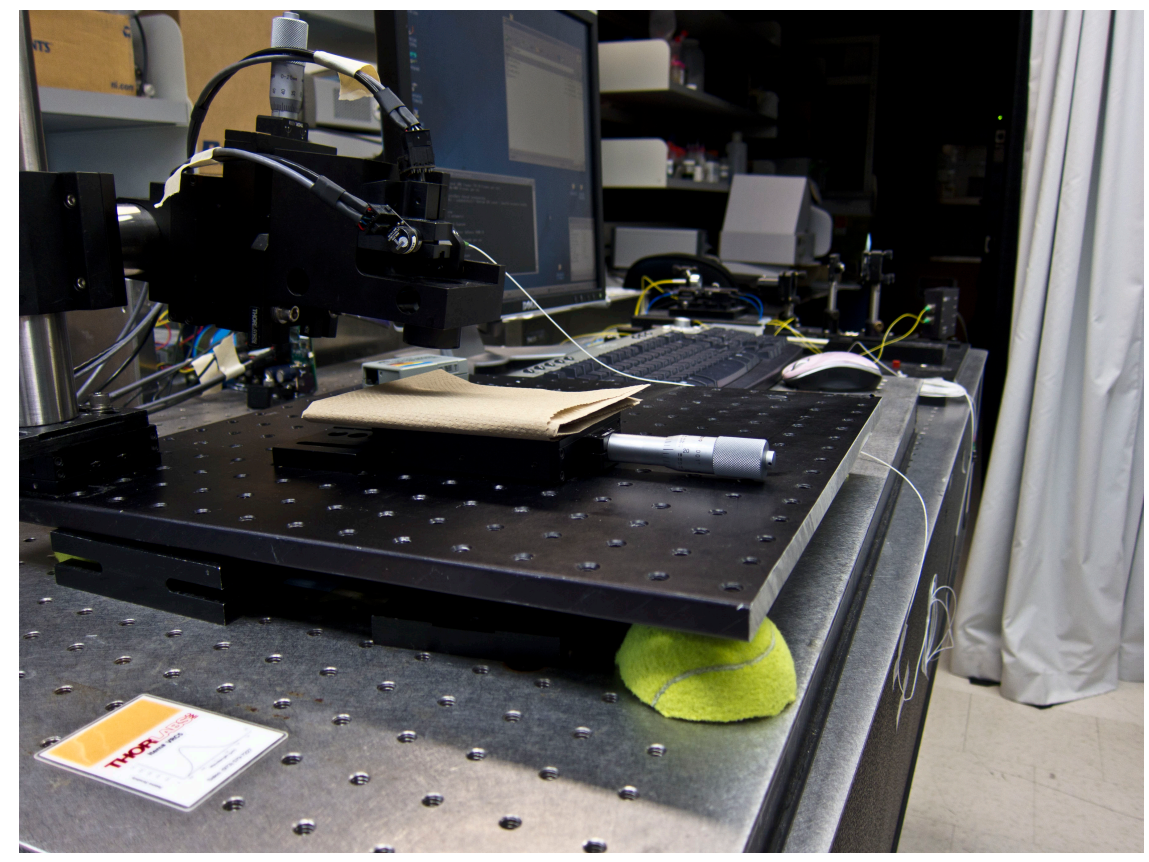

Figure B-1: SS-OCT bench-top setup at Princess Margaret Hospital.

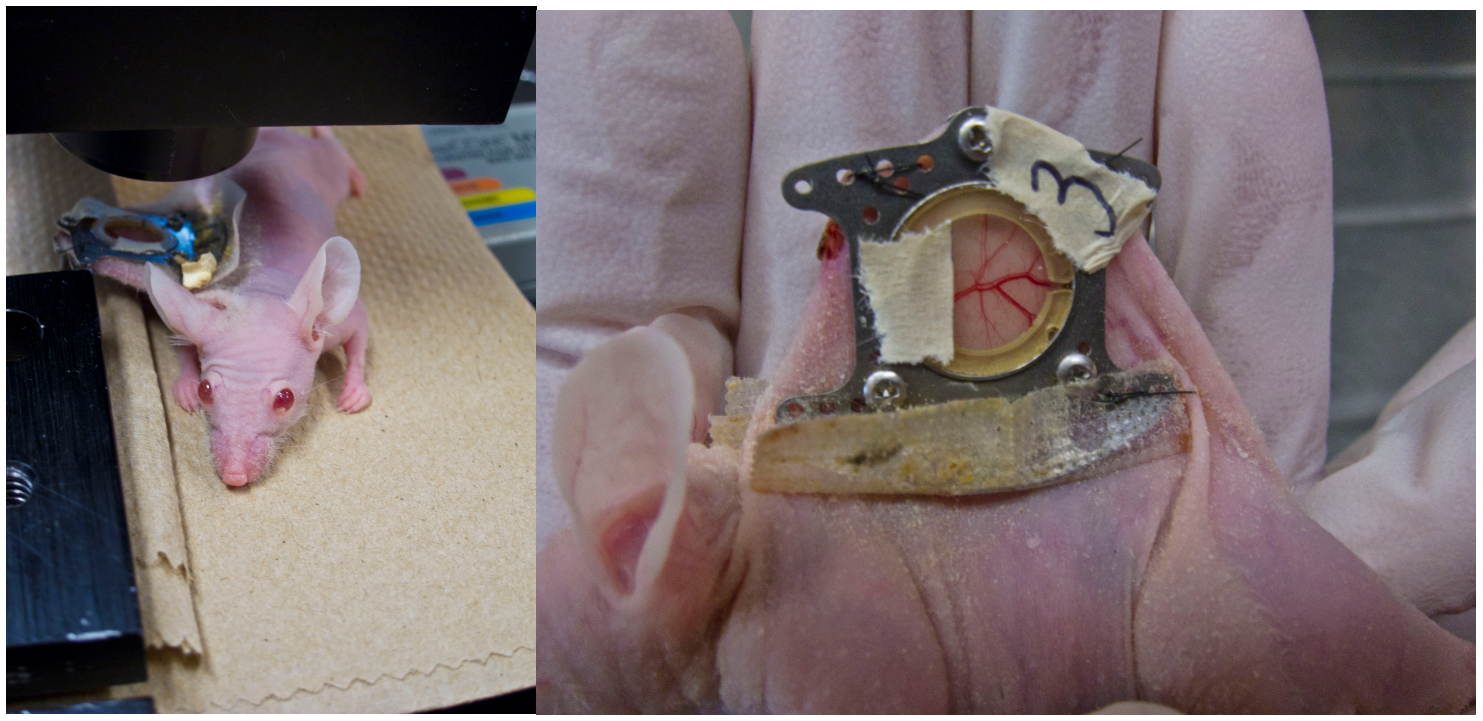

Figure B-2: (left) Dorsal skin window chamber mouse tumour imaging with the SS-OCT system; (right) Dorsal skin window chamber on a mouse. 


\section{Appendix C: Sample MATLAB Code for Formulating DT Maps}

The following function processes the structural file " $\mathrm{S}$ " to produce the decorrelation time maps desired. The specific outputs of this function are the DT map (without averaging), the DT map (with averaging), the power spectrum autocorrelation function, and the heterodyne intermediate scattering function.

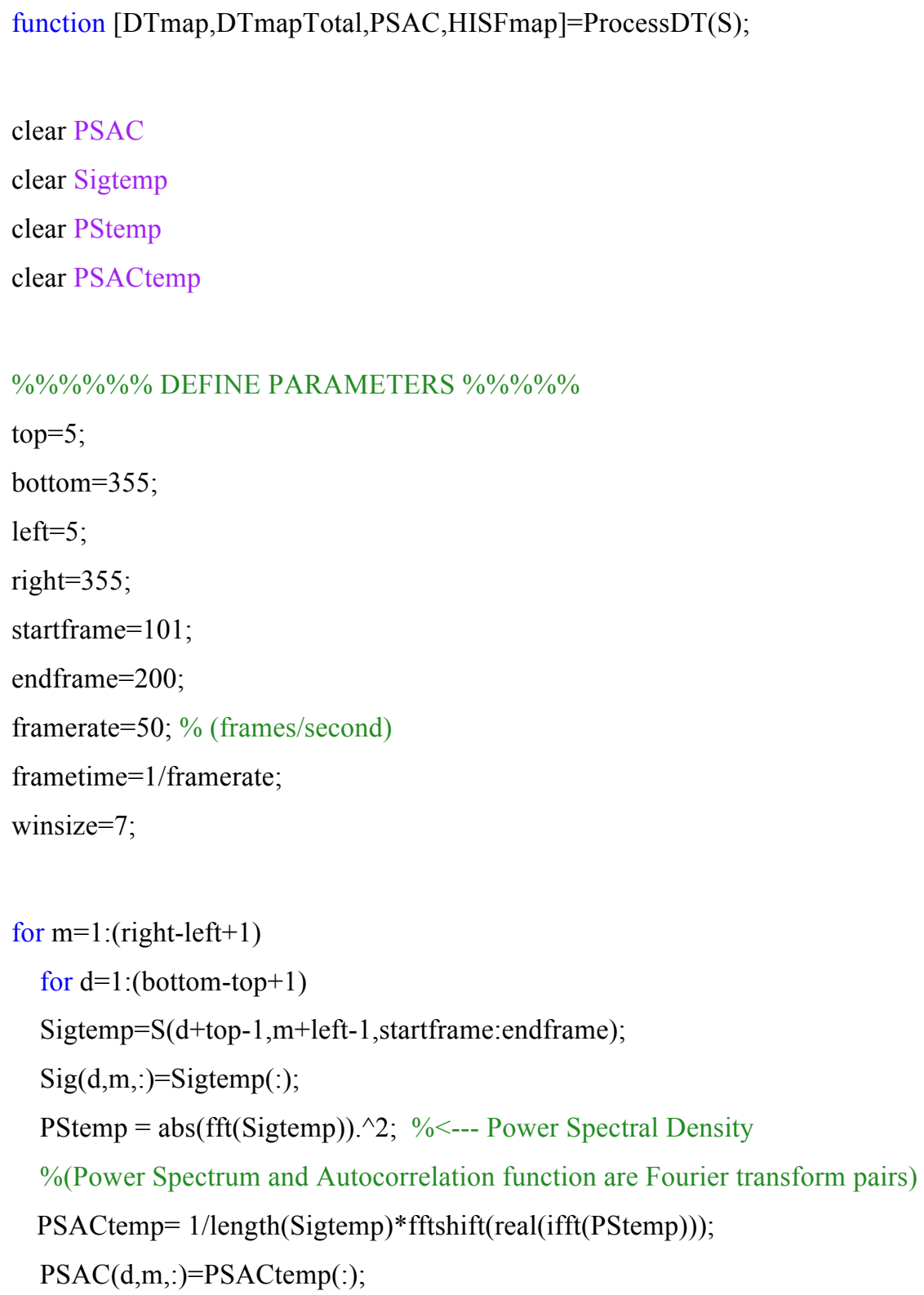




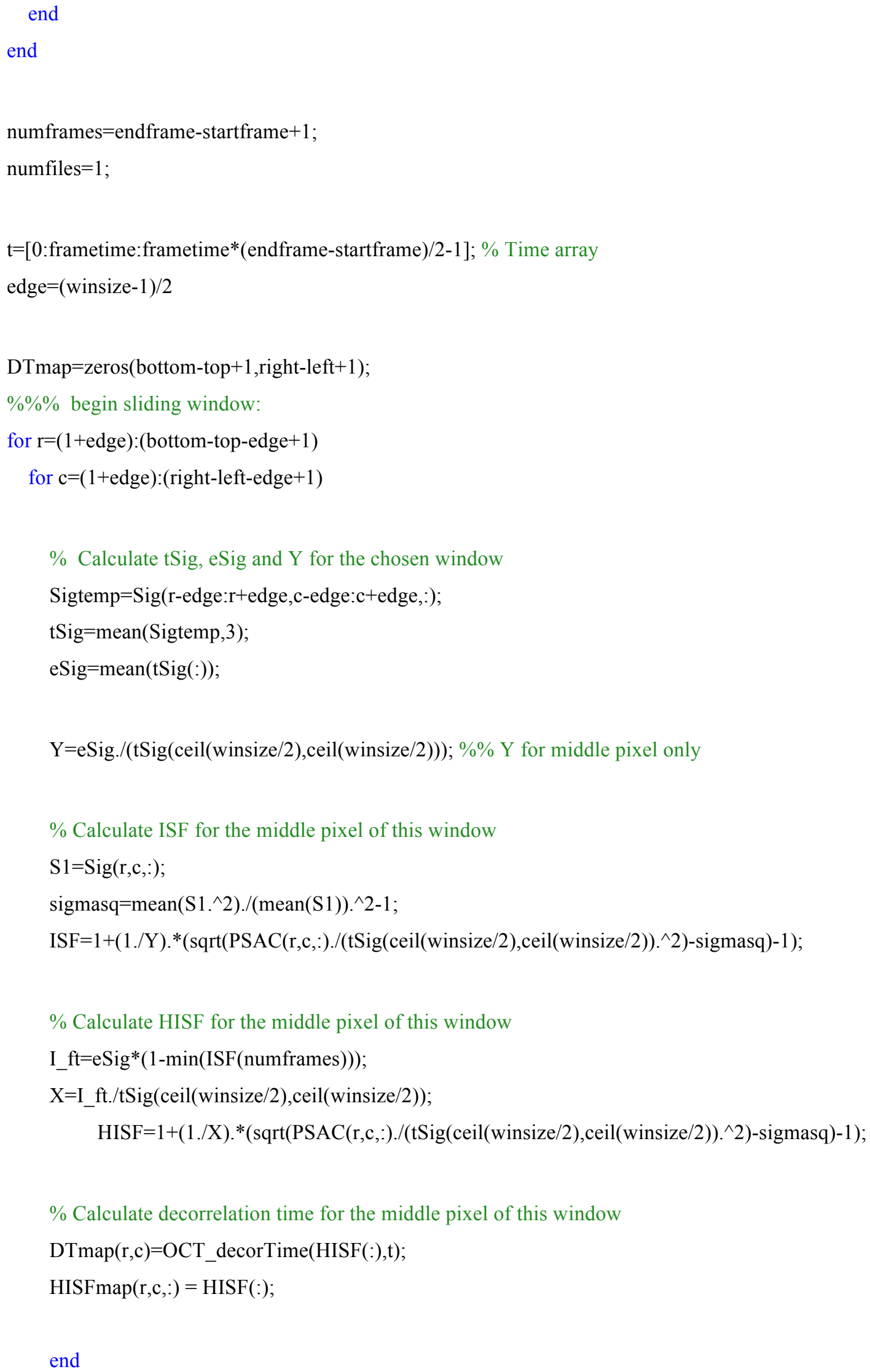




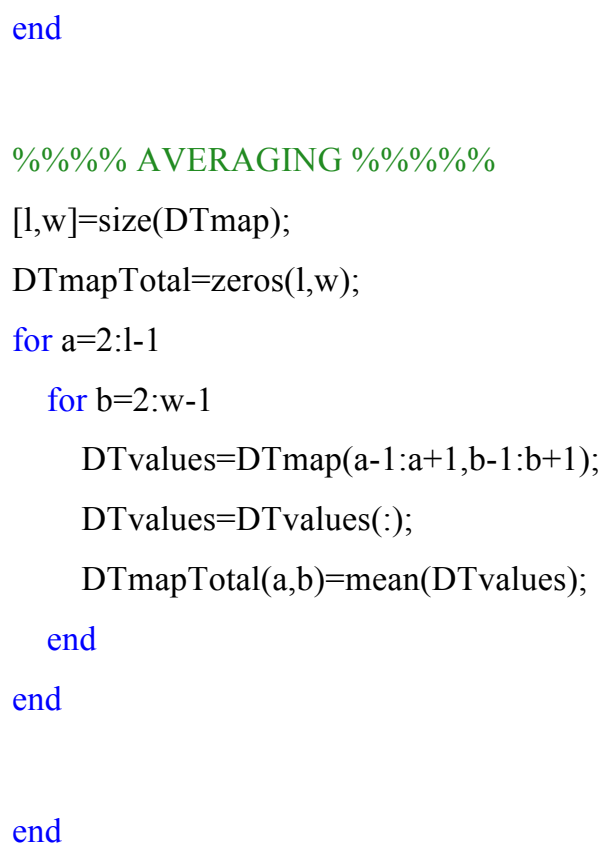




\section{Appendix D: Heterodyne Approach}

In order to adequately outline the heterodyne approach described by Joosten et al. [45] and understand ergodicity, it is important to differentiate between time average and ensemble average.

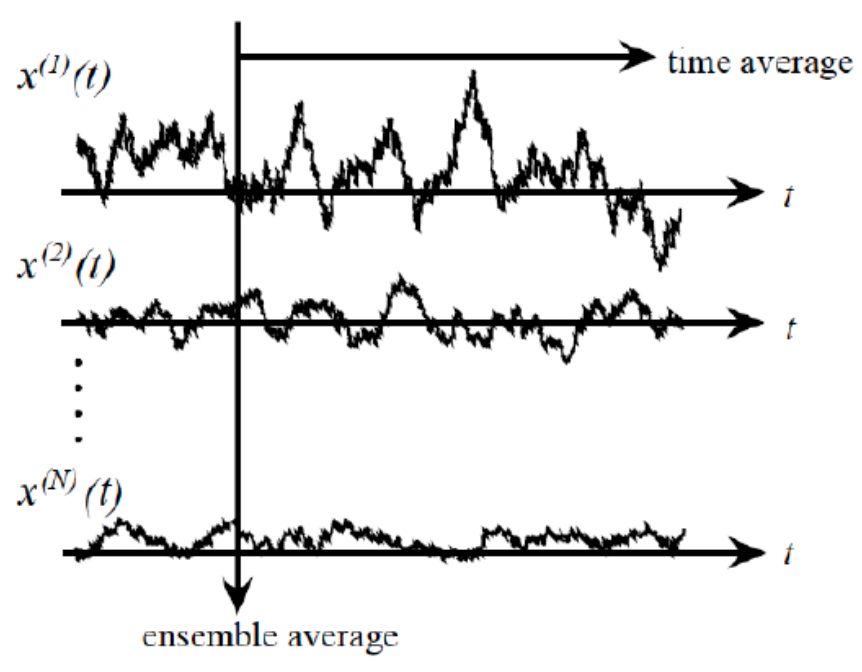

Figure D-1: Time average vs. ensemble average [51].

Time average refers to the average quantity of a single system over a time interval.

Ensemble average refers to the averaged quantity of many identical systems at a certain time. Therefore, it is possible to obtain two different types of results for a data set: one statistical analysis for one signal/pixel over a certain period of time (time average), versus one statistical analysis over the entire ensemble of signals/pixels at a certain moment of time (ensemble average).

Ergodicity is a term used to describe whether or not a dynamic system or process has every sample as an equal representative of the whole. In other words, if a system is 
ergodic, then every individual signal or sample is also a representative of all of the signals. An ergodic system is when time average and ensemble average give the same result.

The heterodyne approach used in the thesis firstly assumes that the tumour tissues are nonergodic media. This is the right assessment because similar to the gel medium described by Joosten et al. [45], the time-averaged measurements of the tumour tissue will not in general be equivalent to an ensemble-averaged measurement. This is because a tumour tissue will have different cell types and cells in various stages of tumour growth, so taking the signals at one location is not a representative of the whole.

There is a fundamental difference described by Joosten et al. [45] between the nonergodic and heterodyne approaches. Both are used to measure an inhomogeneous system, but the heterodyne approach assumes that the static inhomogeneities do not constitute a relevant part of the system, but the nonergodic situation inherently takes into account all the features of the system without making any assumptions on what is relevant or irrelevant. The heterodyne approach separates the light scattering from the system into contributions from concentration fluctuations of the network and contributions from static macroscopic inhomogeneities. Only the fluctuating signals detected are assumed relevant, whereas the static signals are not considered. 
The following steps and equations correspond with the MATLAB code presented in Appendix C.

The decorrelation time for each pixel of each imaging plane was determined by calculating the autocorrelation function of the fluctuating signal by taking the inverse Fourier transform of the power spectrum. (Note: the autocorrelation function and the signal's power spectrum are Fourier transform pairs.) The autocorrelation function, $\phi(q, \tau)$, is:

$\phi(q, \tau)=\overline{I(q, t) I(q, t+\tau)}=\lim _{T \rightarrow \infty} \frac{1}{T} \int_{-\frac{T}{2}}^{\frac{T}{2}} I(q, t) I(q, t+\tau) d t$

Eq.D-1

The time-averaged normalized autocorrelation function, $g_{T}{ }^{(2)}(q, \tau)$, is calculated as:

$g_{T}^{(2)}(q, \tau) \equiv \frac{\phi(q, \tau)}{\langle I(q, 0)\rangle_{T}{ }^{2}}=\frac{\langle I(q, 0) I(q, \tau)\rangle_{T}}{\langle I(q, 0)\rangle_{T}{ }^{2}}$

The heterodyne approach removes the static scattering contribution of the 7 by 7 pixel region from the intensity autocorrelation function to produce the heterodyne intermediate scattering function (ISF).

For a chosen 7 by 7 pixel window, the time-averaged normalized autocorrelation function in Eq.D-2 is used to calculate the ISF, $f(q, \tau)$ :

$f(q, \tau) \equiv 1+\frac{1}{Y}\left[\sqrt{g_{T}^{(2)}(q, \tau)-\sigma_{I}^{2}}-1\right]$

where $\mathrm{Y}$ is the ratio between the ensemble average intensity over the time average intensity: 
$Y \equiv \frac{\langle I(q)\rangle_{E}}{\langle I(q)\rangle_{T}}$

Eq.D-4

and $\sigma_{I}^{2}$ is the mean-square intensity fluctuation:

$\sigma_{I}^{2} \equiv \frac{\left\langle I^{2}(q)\right\rangle_{T}}{\langle I(q)\rangle_{T}{ }^{2}}-1=g_{T}{ }^{(2)}(q, 0)-1$

Eq.D-5

The heterodyne ISF equation is similar to Eq.D-3, except the ratio $\mathrm{X}$ is used instead of $\mathrm{Y}$ :

$f_{N}(q, \tau)=1+\frac{1}{X}\left[\sqrt{g_{T}^{(2)}(q, \tau)-\sigma_{I}^{2}}-1\right]$

Eq.D-6

where $\mathrm{X}$ is the ratio between the time average fluctuating intensity over the time average

intensity:

$X \equiv \frac{\left\langle I_{F}(q)\right\rangle_{T}}{\langle I(q)\rangle_{T}}$

Eq.D-7

The 1/e value of the heterodyne ISF was then used to calculate the DT that was displayed in the DT map, after which 3 by 3 spatial averaging is applied to produce the final DT map. 


\section{Appendix E: Threshold Charts}

The following two summary charts correspond to Tables 4-2 and 4-3. The chart data for average DT, difference, and pixel count for each threshold are presented for Mouse 6 and Mouse 10.

Table E-1: A full summary chart of the data corresponding to Table 4-2 for Mouse 6 from Day 0 to Day 2. Difference refers to the increase in average DT compared to the next longest DT threshold.

\begin{tabular}{|c|c|c|c|c|c|c|c|c|c|c|c|c|c|c|}
\hline \multicolumn{5}{|c|}{ Day 0} & \multicolumn{5}{|c|}{ Day 1} & \multicolumn{5}{|c|}{ Day 2} \\
\hline Plane & Thres & AvgDT & Diff & PixelCount & Plane & Thres & AvgDT & Diff & PixelCount & Plane & Thres & AvgDT & Diff & PixelCount \\
\hline \multirow[t]{5}{*}{8} & 20 & 36 & 22 & 1500 & 6 & 20 & 46 & 9 & 1500 & 5 & 20 & 58 & 23 & 1500 \\
\hline & 30 & 58 & 11 & 582 & & 30 & 55 & 8 & 1065 & & 30 & 81 & 10 & 915 \\
\hline & 40 & 69 & 10 & 401 & & 40 & 63 & 10 & 769 & & 40 & 91 & 10 & 755 \\
\hline & 50 & 79 & 7 & 290 & & 50 & 73 & 8 & 504 & & 50 & 101 & 9 & 624 \\
\hline & 60 & 86 & & 223 & & 60 & 81 & & 350 & & 60 & 110 & & 527 \\
\hline \multirow[t]{5}{*}{9} & 20 & 36 & 25 & 1500 & 7 & 20 & 58 & 11 & 1500 & 6 & 20 & 59 & 22 & 1500 \\
\hline & 30 & 61 & 8 & 555 & & 30 & 69 & 7 & 1125 & & 30 & 81 & 10 & 942 \\
\hline & 40 & 69 & 14 & 428 & & 40 & 76 & 9 & 939 & & 40 & 91 & 8 & 775 \\
\hline & 50 & 83 & 9 & 280 & & 50 & 85 & 7 & 741 & & 50 & 99 & 9 & 662 \\
\hline & 60 & 92 & & 211 & & 60 & 92 & & 596 & & 60 & 108 & & 555 \\
\hline \multirow[t]{5}{*}{10} & 20 & 32 & 17 & 1500 & 8 & 20 & 42 & 14 & 1500 & 7 & 20 & 83 & 27 & 1500 \\
\hline & 30 & 49 & 8 & 554 & & 30 & 56 & 10 & 843 & & 30 & 110 & 10 & 1042 \\
\hline & 40 & 57 & 12 & 358 & & 40 & 66 & 11 & 577 & & 40 & 120 & 9 & 923 \\
\hline & 50 & 69 & 9 & 198 & & 50 & 77 & 10 & 380 & & 50 & 129 & 10 & 819 \\
\hline & 60 & 78 & & 121 & & 60 & 87 & & 270 & & 60 & 139 & & 728 \\
\hline \multirow[t]{5}{*}{11} & 20 & 35 & 20 & 1500 & 9 & 20 & 36 & 15 & 1500 & 8 & 20 & 64 & 16 & 1500 \\
\hline & 30 & 55 & 8 & 558 & & 30 & 51 & 12 & 706 & & 30 & 80 & 9 & 1068 \\
\hline & 40 & 63 & 11 & 402 & & 40 & 63 & 13 & 427 & & 40 & 89 & 9 & 890 \\
\hline & 50 & 74 & 9 & 258 & & 50 & 76 & 10 & 244 & & 50 & 98 & 7 & 743 \\
\hline & 60 & 83 & & 176 & & 60 & 86 & & 171 & & 60 & 105 & & 646 \\
\hline \multirow[t]{5}{*}{12} & 20 & 42 & 18 & 1500 & & & & & & 9 & 20 & 83 & 16 & 1500 \\
\hline & 30 & 60 & 7 & 787 & & & & & & & 30 & 99 & 9 & 1182 \\
\hline & 40 & 67 & 11 & 608 & & & & & & & 40 & 108 & 9 & 1037 \\
\hline & 50 & 78 & 6 & 419 & & & & & & & 50 & 117 & 6 & 910 \\
\hline & 60 & 84 & & 330 & & & & & & & 60 & 123 & & 829 \\
\hline \multirow[t]{5}{*}{13} & 20 & 41 & 16 & 1500 & & & & & & 10 & 20 & 72 & 19 & 1500 \\
\hline & 30 & 57 & 7 & 831 & & & & & & & 30 & 91 & 11 & 1074 \\
\hline & 40 & 64 & 8 & 640 & & & & & & & 40 & 102 & 9 & 912 \\
\hline & 50 & 72 & 7 & 453 & & & & & & & 50 & 111 & 8 & 783 \\
\hline & 60 & 79 & & 320 & & & & & & & 60 & 119 & & 686 \\
\hline \multirow[t]{5}{*}{14} & 20 & 69 & 15 & 1500 & & & & & & 11 & 20 & 89 & 24 & 1500 \\
\hline & 30 & 84 & 10 & 1124 & & & & & & & 30 & 113 & 10 & 1100 \\
\hline & 40 & 94 & 11 & 930 & & & & & & & 40 & 123 & 9 & 968 \\
\hline & 50 & 105 & 8 & 771 & & & & & & & 50 & 132 & 8 & 875 \\
\hline & 60 & 113 & & 668 & & & & & & & 60 & 140 & & 792 \\
\hline \multirow[t]{5}{*}{15} & 20 & 46 & 16 & 1500 & & & & & & 12 & 20 & 73 & 20 & 1500 \\
\hline & 30 & 62 & 58 & 906 & & & & & & & 30 & 93 & 8 & 1085 \\
\hline & 40 & 120 & 26 & 758 & & & & & & & 40 & 101 & 9 & 958 \\
\hline & 50 & 146 & 18 & 562 & & & & & & & 50 & 110 & 9 & 827 \\
\hline & 60 & 164 & & 469 & & & & & & & 60 & 119 & & 710 \\
\hline
\end{tabular}


Table E-2: A full summary chart of the data corresponding to Table 4-3 for Mouse 10 from Day 0 to Day 2. Difference refers to the increase in average DT compared to the next longest DT threshold.

\begin{tabular}{|c|c|c|c|c|c|c|c|c|c|c|c|c|c|c|}
\hline \multicolumn{5}{|c|}{ Day 0} & \multicolumn{5}{|c|}{ Day 1} & \multicolumn{5}{|c|}{ Day 2} \\
\hline Plane & Thres & AvgDT & Diff & PixelCount & Plane & Thres & AvgDT & Diff & PixelCount & Plane & Thres & AvgDT & Diff & PixelCoun \\
\hline \multirow[t]{5}{*}{0} & 20 & 23 & 14 & 9000 & 0 & 20 & 23 & 14 & 9000 & 0 & 20 & 42 & 19 & 9000 \\
\hline & 30 & 37 & 10 & 725 & & 30 & 37 & 9 & 848 & & 30 & 61 & 7 & 4706 \\
\hline & 40 & 47 & 14 & 176 & & 40 & 46 & 9 & 247 & & 40 & 68 & 11 & 3649 \\
\hline & 50 & 61 & 9 & 38 & & 50 & 55 & 7 & 50 & & 50 & 79 & 8 & 2523 \\
\hline & 60 & 70 & & 17 & & 60 & 62 & & 8 & & 60 & 87 & & 1921 \\
\hline \multirow[t]{5}{*}{1} & 20 & 25 & 19 & 9000 & 1 & 20 & 25 & 17 & 9000 & 1 & 20 & 44 & 18 & 9000 \\
\hline & 30 & 44 & 11 & 1353 & & 30 & 42 & 8 & 1728 & & 30 & 62 & 11 & 4769 \\
\hline & 40 & 55 & 14 & 647 & & 40 & 50 & 11 & 820 & & 40 & 73 & 12 & 3482 \\
\hline & 50 & 69 & 11 & 295 & & 50 & 61 & 11 & 300 & & 50 & 85 & 9 & 2485 \\
\hline & 60 & 80 & & 177 & & 60 & 72 & & 121 & & 60 & 94 & & 1901 \\
\hline \multirow[t]{5}{*}{2} & 20 & 25 & 18 & 9000 & 2 & 20 & 27 & 15 & 9000 & 2 & 20 & 36 & 17 & 9000 \\
\hline & 30 & 43 & 12 & 1181 & & 30 & 42 & 7 & 2316 & & 30 & 53 & 8 & 3899 \\
\hline & 40 & 55 & 15 & 516 & & 40 & 49 & 12 & 1148 & & 40 & 61 & 10 & 2802 \\
\hline & 50 & 70 & 11 & 228 & & 50 & 61 & 9 & 377 & & 50 & 71 & 8 & 1734 \\
\hline & 60 & 81 & & 136 & & 60 & 70 & & 166 & & 60 & 79 & & 1184 \\
\hline \multirow[t]{5}{*}{3} & 20 & 24 & 16 & 9000 & 3 & 20 & 27 & 15 & 9000 & 3 & 20 & 53 & 22 & 9000 \\
\hline & 30 & 40 & 10 & 1240 & & 30 & 42 & 9 & 2418 & & 30 & 75 & 9 & 5270 \\
\hline & 40 & 50 & 12 & 467 & & 40 & 51 & 10 & 1139 & & 40 & 84 & 10 & 4284 \\
\hline & 50 & 62 & 8 & 171 & & 50 & 61 & 9 & 470 & & 50 & 94 & 7 & 3459 \\
\hline & 60 & 70 & & 83 & & 60 & 70 & & 221 & & 60 & 101 & & 2945 \\
\hline \multirow[t]{5}{*}{4} & 20 & 27 & 18 & 9000 & 4 & 20 & 26 & 14 & 9000 & 4 & 20 & 49 & 24 & 9000 \\
\hline & 30 & 45 & 10 & 2112 & & 30 & 40 & 8 & 2128 & & 30 & 73 & 9 & 4681 \\
\hline & 40 & 55 & 13 & 1109 & & 40 & 48 & 11 & 860 & & 40 & 82 & 9 & 3879 \\
\hline & 50 & 68 & 12 & 517 & & 50 & 59 & 11 & 269 & & 50 & 91 & 8 & 3125 \\
\hline & 60 & 80 & & 277 & & 60 & 70 & & 97 & & 60 & 99 & & 2558 \\
\hline \multirow[t]{5}{*}{5} & 20 & 24 & 15 & 9000 & 5 & 20 & 30 & 12 & 9000 & 5 & 20 & 56 & 26 & 9000 \\
\hline & 30 & 39 & 11 & 1130 & & 30 & 42 & 8 & 3369 & & 30 & 82 & 9 & 5027 \\
\hline & 40 & 50 & 16 & 377 & & 40 & 50 & 10 & 11714 & & 40 & 91 & 8 & 4292 \\
\hline & 50 & 66 & 9 & 113 & & 50 & 60 & 8 & 689 & & 50 & 99 & 7 & 3627 \\
\hline & 60 & 75 & & 67 & & 60 & 68 & & 286 & & 60 & 106 & & 3153 \\
\hline \multirow[t]{5}{*}{6} & 20 & 23 & 14 & 9000 & 6 & 20 & 32 & 12 & 9000 & 6 & 20 & 52 & 23 & 9000 \\
\hline & 30 & 37 & 10 & 665 & & 30 & 44 & 8 & 3770 & & 30 & 75 & 9 & 5133 \\
\hline & 40 & 47 & 10 & 194 & & 40 & 52 & 10 & 2050 & & 40 & 84 & 11 & 4197 \\
\hline & 50 & 57 & 7 & 53 & & 50 & 62 & 10 & 883 & & 50 & 95 & 8 & 3302 \\
\hline & 60 & 64 & & 16 & & 60 & 72 & & 409 & & 60 & 103 & & 2736 \\
\hline \multirow[t]{5}{*}{7} & 20 & 25 & 18 & 9000 & 7 & 20 & 34 & 13 & 9000 & 7 & 20 & 34 & 17 & 9000 \\
\hline & 30 & 43 & 10 & 1420 & & 30 & 47 & 8 & 4197 & & 30 & 51 & 9 & 3638 \\
\hline & 40 & 53 & 12 & 677 & & 40 & 55 & 9 & 2607 & & 40 & 60 & 12 & 2398 \\
\hline & 50 & 65 & 8 & 310 & & 50 & 64 & 8 & 1392 & & 50 & 72 & 8 & 1384 \\
\hline & 60 & 73 & & 173 & & 60 & 72 & & 788 & & 60 & 80 & & 939 \\
\hline \multirow[t]{5}{*}{8} & 20 & 24 & 17 & 9000 & 8 & 20 & 36 & 12 & 9000 & 8 & 20 & 38 & 12 & 9000 \\
\hline & 30 & 41 & 11 & 1012 & & 30 & 48 & 8 & 4741 & & 30 & 60 & 9 & 3703 \\
\hline & 40 & 52 & 11 & 387 & & 40 & 56 & 11 & 3041 & & 40 & 69 & 11 & 2797 \\
\hline & 50 & 63 & 8 & 170 & & 50 & 67 & 7 & 1649 & & 50 & 80 & 8 & 1937 \\
\hline & 60 & 71 & & 96 & & 60 & 74 & & 1021 & & 60 & 88 & & 1451 \\
\hline \multirow[t]{5}{*}{9} & 20 & 22 & 16 & 9000 & 9 & 20 & 36 & 13 & 9000 & 9 & 20 & 38 & 12 & 9000 \\
\hline & 30 & 38 & 9 & 563 & & 30 & 49 & 8 & 4433 & & 30 & 60 & 8 & 3901 \\
\hline & 40 & 47 & 12 & 174 & & 40 & 57 & 10 & 2928 & & 40 & 68 & 10 & 2971 \\
\hline & 50 & 59 & 7 & 50 & & 50 & 67 & 8 & 1684 & & 50 & 78 & 9 & 2076 \\
\hline & 60 & 66 & & 19 & & 60 & 75 & & 1020 & & 60 & 87 & & 1526 \\
\hline 10 & 20 & 23 & 17 & 9000 & 10 & 20 & 37 & 13 & 9000 & 10 & 20 & 43 & 23 & 9000 \\
\hline & 30 & 40 & 10 & 852 & & 30 & 50 & 7 & 4709 & & 30 & 66 & 8 & 4441 \\
\hline & 40 & 50 & 10 & 308 & & 40 & 57 & 10 & 3165 & & 40 & 74 & 11 & 3510 \\
\hline & 50 & 60 & 7 & 116 & & 50 & 67 & 8 & 1804 & & 50 & 85 & 8 & 2573 \\
\hline & 60 & 67 & & 54 & & 60 & 75 & & 1095 & & 60 & 93 & & 2042 \\
\hline 11 & 20 & 24 & 22 & 9000 & 11 & 20 & 34 & 15 & 9000 & 11 & 20 & 39 & 23 & 9000 \\
\hline
\end{tabular}




\begin{tabular}{|c|c|c|c|c|c|c|c|c|c|c|c|c|c|c|}
\hline & 30 & 46 & 15 & 1003 & & 30 & 49 & 8 & 4066 & & 30 & 62 & 8 & 3803 \\
\hline & 40 & 61 & 15 & 442 & & 40 & 57 & 10 & 2636 & & 40 & 70 & 11 & 2969 \\
\hline & 50 & 76 & 11 & 238 & & 50 & 67 & 7 & 1528 & & 50 & 81 & 8 & 2092 \\
\hline & 60 & 87 & & 161 & & 60 & 74 & & 976 & & 60 & 89 & & 1584 \\
\hline \multirow[t]{5}{*}{12} & 20 & 23 & 16 & 9000 & 12 & 20 & 37 & 15 & 9000 & 12 & 20 & 54 & 26 & 9000 \\
\hline & 30 & 39 & 9 & 780 & & 30 & 52 & 8 & 4363 & & 30 & 80 & 8 & 4976 \\
\hline & 40 & 48 & 11 & 302 & & 40 & 60 & 10 & 2928 & & 40 & 88 & 11 & 4242 \\
\hline & 50 & 59 & 8 & 94 & & 50 & 70 & 7 & 1818 & & 50 & 99 & 8 & 3398 \\
\hline & 60 & 67 & & 35 & & 60 & 77 & & 1228 & & 60 & 107 & & 2899 \\
\hline \multirow[t]{5}{*}{13} & 20 & 24 & 21 & 9000 & 13 & 20 & 38 & 12 & 9000 & 13 & 20 & 49 & 27 & 9000 \\
\hline & 30 & 45 & 10 & 1190 & & 30 & 50 & 7 & 4943 & & 30 & 76 & 9 & 4547 \\
\hline & 40 & 55 & 12 & 601 & & 40 & 57 & 9 & 3371 & & 40 & 85 & 9 & 3746 \\
\hline & 50 & 67 & 9 & 299 & & 50 & 66 & 8 & 1978 & & 50 & 94 & 8 & 3037 \\
\hline & 60 & 76 & & 178 & & 60 & 74 & & 1214 & & 60 & 102 & & 2530 \\
\hline \multirow[t]{5}{*}{14} & 20 & 24 & 18 & 9000 & 14 & 20 & 38 & 14 & 9000 & 14 & 20 & 38 & 21 & 9000 \\
\hline & 30 & 42 & 13 & 1084 & & 30 & 52 & 7 & 4849 & & 30 & 59 & 9 & 3855 \\
\hline & 40 & 55 & 11 & 445 & & 40 & 59 & 10 & 3453 & & 40 & 68 & 10 & 2887 \\
\hline & 50 & 66 & 8 & 218 & & 50 & 69 & 8 & 2105 & & 50 & 78 & 9 & 1994 \\
\hline & 60 & 74 & & 125 & & 60 & 77 & & 1412 & & 60 & 87 & & 1461 \\
\hline \multirow[t]{5}{*}{15} & 20 & 22 & 18 & 9000 & 15 & 20 & 38 & 13 & 9000 & 15 & 20 & 51 & 21 & 9000 \\
\hline & 30 & 40 & 11 & 583 & & 30 & 51 & 8 & 4835 & & 30 & 72 & 11 & 5091 \\
\hline & 40 & 51 & 12 & 204 & & 40 & 59 & 10 & 3276 & & 40 & 83 & 10 & 3988 \\
\hline & 50 & 63 & 10 & 80 & & 50 & 69 & 7 & 1976 & & 50 & 93 & 9 & 3120 \\
\hline & 60 & 73 & & 39 & & 60 & 76 & & 1299 & & 60 & 102 & & 2563 \\
\hline \multirow[t]{5}{*}{16} & 20 & 24 & 23 & 9000 & 16 & 20 & 38 & 13 & 9000 & 16 & 20 & 39 & 21 & 9000 \\
\hline & 30 & 47 & 13 & 913 & & 30 & 51 & 8 & 4752 & & 30 & 60 & 9 & 3967 \\
\hline & 40 & 60 & 12 & 485 & & 40 & 59 & 10 & 3274 & & 40 & 69 & 11 & 2958 \\
\hline & 50 & 72 & 8 & 269 & & 50 & 69 & 7 & 2037 & & 50 & 80 & 8 & 2087 \\
\hline & 60 & 80 & & 190 & & 60 & 76 & & 1325 & & 60 & 88 & & 1567 \\
\hline \multirow[t]{5}{*}{17} & 20 & 25 & 24 & 9000 & 17 & 20 & 39 & 12 & 9000 & 17 & 20 & 46 & 19 & 9000 \\
\hline & 30 & 49 & 11 & 1158 & & 30 & 51 & 7 & 5212 & & 30 & 65 & 8 & 4938 \\
\hline & 40 & 60 & 13 & 656 & & 40 & 58 & 9 & 3701 & & 40 & 73 & 11 & 3879 \\
\hline & 50 & 73 & 10 & 367 & & 50 & 67 & 7 & 2326 & & 50 & 84 & 9 & 2843 \\
\hline & 60 & 83 & & 247 & & 60 & 74 & & 1490 & & 60 & 93 & & 2176 \\
\hline \multirow[t]{5}{*}{18} & 20 & 26 & 18 & 9000 & 18 & 20 & 40 & 12 & 9000 & 18 & 20 & 36 & 17 & 9000 \\
\hline & 30 & 44 & 11 & 1569 & & 30 & 52 & 8 & 5252 & & 30 & 53 & 8 & 4020 \\
\hline & 40 & 55 & 14 & 758 & & 40 & 60 & 9 & 3683 & & 40 & 61 & 11 & 2846 \\
\hline & 50 & 69 & 11 & 348 & & 50 & 69 & 8 & 2304 & & 50 & 72 & 9 & 1762 \\
\hline & 60 & 80 & & 206 & & 60 & 77 & & 1521 & & 60 & 81 & & 1203 \\
\hline \multirow[t]{5}{*}{19} & 20 & 26 & 20 & 9000 & 19 & 20 & 41 & 12 & 9000 & 19 & 20 & 47 & 19 & 9000 \\
\hline & 30 & 46 & 13 & 1488 & & 30 & 53 & 7 & 5310 & & 30 & 66 & 8 & 4989 \\
\hline & 40 & 59 & 16 & 719 & & 40 & 60 & 9 & 3882 & & 40 & 74 & 10 & 4018 \\
\hline & 50 & 75 & 14 & 351 & & 50 & 69 & 8 & 2438 & & 50 & 84 & 8 & 3015 \\
\hline & 60 & 89 & & 213 & & 60 & 77 & & 1601 & & 60 & 92 & & 2325 \\
\hline \multirow[t]{5}{*}{20} & 20 & 27 & 20 & 9000 & 20 & 20 & 42 & 11 & 9000 & 20 & 20 & 66 & 25 & 9000 \\
\hline & 30 & 47 & 12 & 1846 & & 30 & 53 & 7 & 5695 & & 30 & 91 & 9 & 5823 \\
\hline & 40 & 59 & 15 & 967 & & 40 & 60 & 9 & 4149 & & 40 & 100 & 9 & 4996 \\
\hline & 50 & 74 & 13 & 493 & & 50 & 69 & 7 & 2692 & & 50 & 109 & 6 & 4329 \\
\hline & 60 & 87 & & 295 & & 60 & 76 & & 1847 & & 60 & 115 & & 3874 \\
\hline \multirow[t]{5}{*}{21} & 20 & 26 & 20 & 9000 & 21 & 20 & 42 & 10 & 9000 & 21 & 20 & 27 & 15 & 9000 \\
\hline & 30 & 46 & 11 & 1845 & & 30 & 52 & 7 & 5800 & & 30 & 42 & 9 & 2136 \\
\hline & 40 & 57 & 11 & 978 & & 40 & 59 & 9 & 4178 & & 40 & 51 & 10 & 1032 \\
\hline & 50 & 68 & 8 & 522 & & 50 & 68 & 7 & 2665 & & 50 & 61 & 9 & 426 \\
\hline & 60 & 76 & & 336 & & 60 & 75 & & 1689 & & 60 & 69 & & 200 \\
\hline \multirow[t]{5}{*}{22} & 20 & 25 & 20 & 9000 & 22 & 20 & 42 & 11 & 9000 & 22 & 20 & 67 & 24 & 9000 \\
\hline & 30 & 45 & 11 & 1445 & & 30 & 53 & 6 & 5729 & & 30 & 91 & 8 & 5870 \\
\hline & 40 & 56 & 13 & 712 & & 40 & 59 & 10 & 4210 & & 40 & 99 & 10 & 5115 \\
\hline & 50 & 69 & 11 & 359 & & 50 & 69 & 7 & 2578 & & 50 & 109 & 9 & 4331 \\
\hline & 60 & 80 & & 203 & & 60 & 76 & & 1703 & & 60 & 118 & & 3714 \\
\hline 23 & 20 & 22 & 15 & 9000 & 23 & 20 & 40 & 11 & 9000 & 23 & 20 & 61 & 22 & 9000 \\
\hline & 30 & 37 & 11 & 460 & & 30 & 51 & 7 & 5311 & & 30 & 83 & 9 & 5689 \\
\hline & 40 & 48 & 13 & 115 & & 40 & 58 & 9 & 3686 & & 40 & 92 & 9 & 4807 \\
\hline
\end{tabular}




\begin{tabular}{|c|c|c|c|c|c|c|c|c|c|c|c|c|c|c|}
\hline & 50 & 61 & 14 & 34 & & 50 & 67 & 8 & 2210 & & 50 & 101 & 8 & 3995 \\
\hline & 60 & 75 & & 12 & & 60 & 75 & & 1400 & & 60 & 109 & & 3416 \\
\hline \multirow[t]{5}{*}{24} & 20 & 25 & 17 & 9000 & 24 & 20 & 38 & 12 & 9000 & 24 & 20 & 48 & 22 & 9000 \\
\hline & 30 & 42 & 11 & 1583 & & 30 & 50 & 7 & 5155 & & 30 & 70 & 7 & 4938 \\
\hline & 40 & 53 & 12 & 689 & & 40 & 57 & 10 & 3466 & & 40 & 77 & 10 & 4025 \\
\hline & 50 & 65 & 9 & 293 & & 50 & 67 & 7 & 1999 & & 50 & 87 & 9 & 3124 \\
\hline & 60 & 74 & & 162 & & 60 & 74 & & 1243 & & 60 & 96 & & 2481 \\
\hline \multirow[t]{5}{*}{25} & 20 & 27 & 21 & 9000 & 25 & 20 & 40 & 11 & 9000 & 25 & 20 & 46 & 21 & 9000 \\
\hline & 30 & 48 & 11 & 1921 & & 30 & 51 & 7 & 5620 & & 30 & 67 & 9 & 4848 \\
\hline & 40 & 59 & 16 & 1064 & & 40 & 58 & 9 & 3892 & & 40 & 76 & 9 & 3866 \\
\hline & 50 & 75 & 10 & 535 & & 50 & 67 & 8 & 2327 & & 50 & 85 & 9 & 2967 \\
\hline & 60 & 85 & & 353 & & 60 & 75 & & 1436 & & 60 & 94 & & 2316 \\
\hline
\end{tabular}

The following summary chart corresponds to Table 4-4 regarding average DT and pixel count values for categories $\mathrm{C} 1, \mathrm{C} 2, \mathrm{C} 3$, and $\mathrm{C} 4$ for mice \#1, 2, 5, 6, and 10.

Table E-3: Actual average DT values and pixel counts for all four categories corresponding to Table 4-4.

\begin{tabular}{|l|l|l|l|l|l|l|}
\hline & Day 0 & Day 0 & Day 1 & Day 1 & Day 2 & Day 2 \\
\hline Average DT & Pixel Count & Average DT & Pixel Count & Average DT & Pixel Count \\
\hline M1; C2 & 53 & 8997 & 44 & 4987 & N/A & N/A \\
\hline M1; C3 & 37 & 299 & 46 & 198 & N/A & N/A \\
\hline M1; C4 & 48 & 20000 & 27 & 19900 & N/A & N/A \\
\hline M2; C1 & 47 & 400 & 35 & 390 & N/A & N/A \\
\hline M2; C2 & 52 & 6641 & 58 & 8918 & 62 & 12301 \\
\hline M2; C3 & 30 & 20000 & 38 & 20000 & 47 & 291 \\
\hline M2; C4 & 36 & 400 & 52 & 400 & 53 & 20000 \\
\hline M5; C1 & 70 & 11180 & 58 & 7823 & 55 & 400 \\
\hline M5; C2 & 101 & 375 & 58 & 246 & 47 & 144 \\
\hline M5; C3 & 48 & 20000 & 36 & 20000 & 35 & 20000 \\
\hline M5; C4 & 96 & 400 & 44 & 400 & 30 & 400 \\
\hline M16; C1 & 56 & 6964 & 47 & 3930 & 73 & 6528 \\
\hline M16; C2 & 73 & 341 & 50 & 146 & 67 & 317 \\
\hline M16; C3 & 34 & 20000 & 26 & 20000 & 38 & 20000 \\
\hline M16; C4 & 65 & 400 & 32 & 400 & 58 & 400 \\
\hline M10; C1 & 53 & 4669 & 47 & 7703 & 79 & 8163 \\
\hline M10; C2 & 99 & 386 & 43 & 206 & 90 & 319 \\
\hline M10; C3 & 29 & 20000 & 32 & 20000 & 45 & 20000 \\
\hline & & 58 & & 64 & \\
\hline
\end{tabular}


The following summary chart corresponds to Table 4-5 regarding the Student's t-test to compare the means of the average DT values of control mice (\#1 and 2) and cisplatin mice ( $\# 5,6$ and 10) for categories $\mathrm{C} 1, \mathrm{C} 2, \mathrm{C} 3$, and $\mathrm{C} 4$.

For the Student's t-test, $\mathrm{H}_{0}=$ the control and cisplatin means are the same (any differences would be random) $\mathrm{H}_{\mathrm{a}}=$ the control and cisplatin means are systematically different Reject $\mathrm{H}_{0}$ if the experimental t-value (t-exp) is greater than the theoretical t-value (t-th) Confidence level $=95 \%(\alpha=0.05)$

The experimental t-values (t-exp) were calculated to be:

$$
t_{\exp }=\frac{\left|\overline{X_{A}}-\overline{X_{B}}\right|}{s_{A B} \sqrt{\frac{1}{n_{A}}+\frac{1}{n_{B}}}}
$$

where $\mathrm{SAB}$ is the pooled estimate of the standard deviation:

$$
s_{A B}=\sqrt{\frac{\left(n_{A}-1\right) s_{A}{ }^{2}+\left(n_{B}-1\right) s_{B}{ }^{2}}{n_{A}+n_{B}-2}}
$$

The theoretical t-values (t-th) were found from t-distribution tables. 
Table E-4: Student's t-test for comparing the means for control and cisplatin mice for all four categories corresponding to Table 4-5.

\begin{tabular}{|c|c|c|c|c|c|c|c|}
\hline \multicolumn{4}{|c|}{ C1 $(n=20000$ for all mice $)$} & \multicolumn{4}{|c|}{ C2 $(n=400$ for all mice $)$} \\
\hline & Day 0 & Day 1 & Day 2 & & Day 0 & Day 1 & Day 2 \\
\hline M1, mean & 53 & 44 & & M1, mean & 57 & 46 & \\
\hline M2, mean & 47 & 58 & 62 & M2, mean & 52 & 58 & 64 \\
\hline $\begin{array}{c}\text { Control } \\
\text { mean }\end{array}$ & 50 & 51 & 62 & $\begin{array}{c}\text { Control } \\
\text { mean }\end{array}$ & 54.5 & 52 & 64 \\
\hline M5, mean & 70 & 58 & 55 & M5, mean & 101 & 58 & 47 \\
\hline M6, mean & 56 & 47 & 73 & M6, mean & 73 & 50 & 67 \\
\hline $\begin{array}{l}\text { M10, } \\
\text { mean }\end{array}$ & 53 & 47 & 79 & $\begin{array}{l}\text { M10, } \\
\text { mean }\end{array}$ & 99 & 43 & 90 \\
\hline $\begin{array}{c}\text { Cisplatin } \\
\text { mean }\end{array}$ & 54.5 & 47 & 76 & $\begin{array}{l}\text { Cisplatin } \\
\text { mean }\end{array}$ & 86 & 46.5 & 78.5 \\
\hline $\begin{array}{l}\text { M1, Std. } \\
\text { Dev. }\end{array}$ & 22 & 15 & & $\begin{array}{l}\text { M1, Std. } \\
\text { Dev. }\end{array}$ & 19 & 14 & \\
\hline $\begin{array}{l}\text { M2, Std. } \\
\text { Dev. }\end{array}$ & 18 & 29 & 27 & $\begin{array}{c}\text { M2, Std. } \\
\text { Dev. }\end{array}$ & 19 & 21 & 25 \\
\hline $\begin{array}{c}\text { Control } \\
\text { Std. Dev. }\end{array}$ & 20.10 & 23.09 & 27 & $\begin{array}{l}\text { Control } \\
\text { Std. Dev. }\end{array}$ & 19 & 17.85 & 25 \\
\hline $\begin{array}{l}\text { M5, Std. } \\
\text { Dev. }\end{array}$ & 37 & 26 & 25 & $\begin{array}{l}\text { M5, Std. } \\
\text { Dev. }\end{array}$ & 49 & 21 & 15 \\
\hline $\begin{array}{c}\text { M6, Std. } \\
\text { Dev. }\end{array}$ & 26 & 19 & 43 & $\begin{array}{c}\text { M6, Std. } \\
\text { Dev. }\end{array}$ & 28 & 21 & 29 \\
\hline $\begin{array}{c}\text { M10, Std. } \\
\text { Dev. }\end{array}$ & 30 & 16 & 52 & $\begin{array}{c}\text { M10, Std. } \\
\text { Dev. }\end{array}$ & 48 & 12 & 50 \\
\hline $\begin{array}{l}\text { Cisplatin } \\
\text { Std. Dev. }\end{array}$ & 31.33 & 20.76 & 41.55 & $\begin{array}{l}\text { Cisplatin } \\
\text { Std. Dev. }\end{array}$ & 42.77 & 18.49 & 34.48 \\
\hline $\begin{array}{l}\text { Pooled } \\
\text { Std. Dev. }\end{array}$ & 28.09 & 21.56 & 41.55 & $\begin{array}{l}\text { Pooled } \\
\text { Std. Dev. }\end{array}$ & 36.61 & 18.28 & 34.48 \\
\hline t-exp & 0.1755 & 0.2032 & 0.2918 & $t-\exp$ & 0.9426 & 0.3296 & 0.3642 \\
\hline df & 3 & 3 & 2 & df & 3 & 3 & 2 \\
\hline t-th & 3.182 & 3.182 & 4.303 & t-th & 3.182 & 3.182 & 4.303 \\
\hline Decision & Retain $\mathrm{H}_{0}$ & Retain $\mathrm{H}_{0}$ & Retain $\mathrm{H}_{0}$ & Decision & Retain $\mathrm{H}_{0}$ & Retain $\mathrm{H}_{0}$ & Retain $\mathrm{H}_{0}$ \\
\hline
\end{tabular}




\begin{tabular}{|c|c|c|c|c|c|c|c|}
\hline \multicolumn{4}{|c|}{ C3 $(n=20000$ for all mice $)$} & \multicolumn{4}{|c|}{ C4 $(n=400$ for all mice) } \\
\hline & Day 0 & Day 1 & Day 2 & & Day 0 & Day 1 & Day 2 \\
\hline M1, mean & 36 & 27 & & M1, mean & 48 & 35 & \\
\hline M2, mean & 30 & 38 & 47 & M2, mean & 36 & 52 & 53 \\
\hline $\begin{array}{l}\text { Control } \\
\text { mean }\end{array}$ & 33 & 32.5 & 47 & $\begin{array}{l}\text { Control } \\
\text { mean }\end{array}$ & 42 & 43.5 & 53 \\
\hline M5, mean & 48 & 36 & 35 & M5, mean & 96 & 44 & 30 \\
\hline M6, mean & 34 & 26 & 38 & M6, mean & 65 & 32 & 58 \\
\hline $\begin{array}{l}\text { M10, } \\
\text { mean }\end{array}$ & 29 & 32 & 45 & $\begin{array}{l}\text { M10, } \\
\text { mean }\end{array}$ & 97 & 34 & 76 \\
\hline $\begin{array}{c}\text { Cisplatin } \\
\text { mean }\end{array}$ & 31.5 & 29 & 41.5 & $\begin{array}{c}\text { Cisplatin } \\
\text { mean }\end{array}$ & 81 & 33 & 67 \\
\hline $\begin{array}{l}\text { M1, Std. } \\
\text { Dev. }\end{array}$ & 21 & 13 & & $\begin{array}{l}\text { M1, Std. } \\
\text { Dev. }\end{array}$ & 22 & 16 & \\
\hline $\begin{array}{l}\text { M2, Std. } \\
\text { Dev. }\end{array}$ & 16 & 27 & 29 & $\begin{array}{l}\text { M2, Std. } \\
\text { Dev. }\end{array}$ & 20 & 23 & 28 \\
\hline $\begin{array}{l}\text { Control } \\
\text { Std. Dev. }\end{array}$ & 18.67 & 21.19 & 29 & $\begin{array}{l}\text { Control } \\
\text { Std. Dev. }\end{array}$ & 21.02 & 19.81 & 36.44 \\
\hline $\begin{array}{l}\text { M5, Std. } \\
\text { Dev. }\end{array}$ & 36 & 24 & 23 & $\begin{array}{l}\text { M5, Std. } \\
\text { Dev. }\end{array}$ & 51 & 24 & 16 \\
\hline $\begin{array}{l}\text { M6, Std. } \\
\text { Dev. }\end{array}$ & 22 & 13 & 34 & $\begin{array}{l}\text { M6, Std. } \\
\text { Dev. }\end{array}$ & 31 & 18 & 32 \\
\hline $\begin{array}{l}\text { M10, Std. } \\
\text { Dev. }\end{array}$ & 20 & 16 & 44 & $\begin{array}{c}\text { M10, Std. } \\
\text { Dev. }\end{array}$ & 49 & 13 & 52 \\
\hline $\begin{array}{l}\text { Cisplatin } \\
\text { Std. Dev. }\end{array}$ & 26.96 & 18.27 & 34.74 & $\begin{array}{l}\text { Cisplatin } \\
\text { Std. Dev. }\end{array}$ & 44.58 & 18.88 & 36.44 \\
\hline $\begin{array}{l}\text { Pooled } \\
\text { Std. Dev. }\end{array}$ & 24.51 & 19.29 & 34.74 & $\begin{array}{l}\text { Pooled } \\
\text { Std. Dev. }\end{array}$ & 38.37 & 19.19 & 36.44 \\
\hline t-exp & 0.0670 & 0.1988 & 0.1371 & t-exp & 1.1134 & 0.5992 & 0.3327 \\
\hline df & 3 & 3 & 2 & df & 3 & 3 & 2 \\
\hline t-th & 3.182 & 3.182 & 4.303 & t-th & 3.182 & 3.182 & 4.303 \\
\hline Decision & Retain $\mathrm{H}_{0}$ & Retain $\mathrm{H}_{0}$ & Retain $\mathrm{H}_{0}$ & Decision & Retain $\mathrm{H}_{0}$ & Retain $\mathrm{H}_{0}$ & Retain $\mathrm{H}_{0}$ \\
\hline
\end{tabular}




\section{Bibliography}

[1] W Drexler, "Ultrahigh-resolution optical coherence tomography," J. Biomed. Opt., vol. 9, no. 1, pp. 47-74, 2004.

[2] JG Fujimoto, BE Bouma, GJ Tearney, "Optical Coherence Tomography:

Introduction," in Handbook of Optical Coherence Tomography, Ed. New York, NY, USA: Marcel Dekker, pp. 1-40, 2002.

[3] B Hermann, M Esmaeelpour, B Považay, B Hofer, F Bounaparte, N Sheen, R North, W Drexler, "Wide field visualization of retinal and choroidal microstructure in vivo frequency domain OCT at $1060 \mathrm{~nm}$ with up to 47000 lines/s," Proc. SPIE Int. Soc. Opt. Eng., vol. 7163, pp.71630A, 2009.

[4] A Unterhuber, B Považay, B Hermann, H Sattmann, A Chavez-Pirson, W Drexler, "In vivo retinal optical coherence tomography at $1040 \mathrm{~nm}$ - enhanced penetration into the choroid," Opt. Express, vol. 13, no. 9, pp. 3252-3258, May 2005.

[5] W Drexler, JG Fujimoto, "State-of-the-art retinal optical coherence tomography," Prog. Retin. Eye Res., vol. 27, pp. 45-88, 2008. 
[6] GJ Tearney, ME Brezinski, BE Bouma, SA Boppart, C Pitris, JF Southern, JG Fujimoto, "In Vivo Endoscopic Optical Biopsy with Optical Coherence Tomography," Science, vol. 276, no. 5321, pp. 2037-2039, 1997.

[7] BA Standish, KKC Lee, A Mariampillai, NR Munce, MKK Leung, VXD Yang, IA Vitkin, "In vivo endoscopic multi-beam optical coherence tomography," Phys. Med. Biol., vol. 55, pp. 615-622, 2010.

[8] Z Chen, W Jung, Y-C Ahn, A Sepehr, WB Armstrong, M Brenner, DT McCormick, NC Tien, "High speed three-dimensional endoscopic OCT using MEMS technology," Proc. SPIE Int. Soc. Opt. Eng., vol. 6466, pp. 64660H, 2007.

[9] MV Sivak Jr, K Kobayashi, JA Izatt, AM Rollins, R Ung-runyawee, A Chak, RCK Wong, GA Isenberg, J Willis, "High-resolution endoscopic imaging of the GI tract using optical coherence tomography," Gastrointest. Endosc., vol. 51, no. 4, pp. 474-479, 2000.

[10] VXD Yang, S-J Tang, ML Gordon, B Qi, G Gardiner, M Cirocco, P Kortan, GB Haber, G Kandel, IA Vitkin, BC Wilson, NE Marcon, “Endoscopic Doppler optical coherence tomography in the human GI tract: initial experience," Gastrointest. Endosc., vol. 61 , no. 7 , pp. 879-890, 2005. 
[11] G Farhat, A Mariampillai, VXD Yang, GJ Czarnota, MC Kolios, “Detecting apoptosis using dynamic light scattering with optical coherence tomography," J. Biomed. Opt., vol. 16, no. 7, pp. 070505, 2011.

[12] G Farhat, A Mariampillai, KKC Lee, VXD Yang, GJ Czarnota, MC Kolios, "Measuring intracellular motion using dynamic light scattering with optical coherence tomography in a mouse tumor model," Proc. SPIE Int. Soc. Opt. Eng., vol. 8230, pp. $823002,2012$.

[13] TD Pollard, "The cytoskeleton, cellular motility and the reductionist agenda," Nature, vol. 422, pp. 741-745, 2003.

[14] D Mizuno, C Tardin, CF Schmidt, FC MacKintosh, “Nonequilibrium Mechanics of Active Cytoskeletal Networks,” Science, vol. 315, pp. 370-373, 2007.

[15] F Julicher, A Ajdari, J Prost, "Modeling molecular motors," Rev. Mod. Phys., vol. 69, no. 4, pp. 1269-1281, 1997.

[16] CP Brangwynne, GH Koenderink, FC MacKintosh, DA Weitz, “Intracellular transport by active diffusion," Trends Cell Biol., vol. 19, no. 9, pp. 423-427, 2009. 
[17] M Suissa, C Place, E Goillot, E Freyssingeas, "Internal dynamics of a living cell nucleus investigated by dynamic light scattering," Eur. Phys. J. E., vol. 26, pp. 435-448, 2008.

[18] C Joo, CL Evans, T Stepinac, T Hasan, JF de Boer, "Diffusive and directional intracellular dynamics measured by field-based dynamic light scattering," Opt. Express, vol. 18 , no. 3, pp. 2858-2871, 2010.

[19] RB Tishler, FD Carlson, "A Study of the Dynamic Properties of the Human Red Blood Cell Membrane Using Quasi-Elastic Light-Scattering Spectroscopy," Biophys. J., vol. 65, pp. 2586-2600, 1993.

[20] A Palmer, TG Mason, JY Xu, SC Kuo, D Wirtz, "Diffusing Wave Spectroscopy Microrheology of Actin Filament Networks," Biophys J., vol. 76, pp. 1063-1071, 1999.

[21] M Heckmeier, SE Skipetrov, G Maret, R Maynard, "Imaging of dynamic heterogeneities in multiple-scattering media," J. Opt. Soc. Am. A, vol. 14, no. 1, pp. 185191, 1997.

[22] J Lee, W Wu, JY Jiang, B Zhu, DA Boas, "Dynamic light scattering optical coherence tomography," Opt. Express, vol. 20, no. 20, pp. 22262-22277, 2012. 
[23] JG Fujimoto, "Optical coherence tomography," Appl. Phys., vol. 2, no. 4, pp. 10991111, July 2001.

[24] AF Fercher, W Drexler, CK Hitzenberger, T Lasser, "Optical Coherence Tomography - Principles and Applications," Rep. Prog. Phys., vol. 66, pp. 239-303, 2003.

[25] A Mauro, "High Speed Rotary System for Catheter Based 3-D Imaging with Optical Coherence Tomography (OCT)," Biomedical Physics, Ryerson University, Toronto, MSc Thesis 2009 .

[26] ME Brezinski, Optical Coherence Tomography: Principles and Applications, Academic Press, Burlington, MA, USA, 2006.

[27] AF Fercher, "Optical coherence tomography - development, principles, applications", Z. Med. Phys., vol. 20, pp. 251-276, 2010.

[28] E Ergun, B Hermann, M Wirtitsch, A Unterhuber, TH Ko, H Sattmann, C Scholda, JG Fujimoto, M Stur, W Drexler, “Assessment of Central Visual Function in Stargardt's Disease/Fundus Flavimaculatus with Ultrahigh-Resolution Optical Coherence Tomography," Invest. Ophthalmol. Vis. Sci., vol. 46, no. 1, pp. 310-316, 2005. 
[29] W Drexler, H Sattmann, B Hermann, TH Ko, M Stur, A Unterhuber, C Scholda, O Findl, M Wirtitsch, JG Fujimoto, AF Fercher, "Enhanced visualization of macular pathology with the use of ultrahigh-resolution optical coherence tomography," Arch Ophthalmol., vol. 121, no. 5, pp. 695-706, 2003.

[30] MG Wirtitsch, E Ergun, B Hermann, A Unterhuber, M Stur, C Scholda, H Sattmann, TH Ko, JG Fujimoto, W Drexler, "Ultrahigh Resolution Optical Coherence Tomography In Macular Dystrophy,” Am J Ophthalmol, vol. 140, no. 6, pp. 976-983, 2005.

[31] G Wollstein, LA Paunescu, TH Ko, JG Fujimoto, A Kowalevicz, I Hartl, S Beaton, H Ishikawa, C Mattox, O Singh, J Duker, W Drexler, JS Schuman, "Ultrahigh-Resolution Optical Coherence Tomography in Glaucoma," Ophthalmology, vol. 112, no. 2, pp. 229$237,2005$.

[32] W Drexler, "Cellular and Functional Optical Coherence Tomography of the Human Retina - The Cogan Lecture," Invest. Ophthalmol. Vis. Sci., vol. 48, no. 12, pp. 5340$5351,2007$.

[33] AG Podoleanu, JA Rogers, DA Jackson, "Three dimensional OCT images from retina and skin," Opt. Express, vol. 7, no. 9, pp. 292-298, 2000. 
[34] M Wojtkowski, V Srinivasan, JG Fujimoto, T Ko, JS Schuman, A Kowalczyk, JS Duker, "Three-dimensional Retinal Imaging with High-Speed Ultrahigh-Resolution Optical Coherence Tomography," Ophthalmology, vol. 112, no. 10, pp. 1734-1746, 2005.

[35] VJ Srinivasan, DC Adler, Y Chen, I Gorczynska, R Huber, JS Duker, JS Schuman, JG Fujimoto, "Ultrahigh-Speed Optical Coherence Tomography for Three-Dimensional and En Face Imaging of the Retina and Optic Nerve Head," Invest. Ophthalmol. Vis. Sci., vol. 49, no. 11, pp. 5103-5110, 2008.

[36] H Yabushita, BE Bouma, SL Houser, HT Aretz, I-K Jang, KH Schlendorf, CR Kauffman, M Shishkov, D-H Kang, EF Halpern, GJ Tearney, “Characterization of Human Atherosclerosis by Optical Coherence Tomography," Circulation, vol. 106, pp. 1640-1645, 2002.

[37] GJ Tearney, H Yabushita, SL Houser, HT Aretz, I-K Jang, KH Schlendorf, CR Kauffman, M Shishkov, EF Halpern, BE Bouma, "Quantification of Macrophage Content in Atherosclerotic Plaques by Optical Coherence Tomography," Circulation, vol. 107, pp. 113-119, 2003.

[38] B Scheller, U Speck, B Romeike, A Schmitt, M Sovak, M Bohm, H-P Stoll, "Contrast media as carriers for local drug delivery," Eur. Heart J., vol. 24, pp. 14621467, 2003. 
[39] M Zimarino, F Prati, E Stabile, J Pizzicannella, T Fouad, A Filippini, R Rabozzi, O Trubiani, G Pizzicannella, R De Caterina, "Optical coherence tomography accurately identifies intermediate atherosclerotic lesions - An in vivo evaluation in the rabbit carotid artery," Atherosclerosis, vol. 193, pp. 94-101, 2007.

[40] A Tanaka, T Imanishi, H Kitabata, T Hubo, S Takarada, T Tanimoto, A Kuroi, H Tsujioka, H Ikejima, S Ueno, H Kataiwa, K Okouchi, M Kashiwaghi, H Matsumoto, K Takemoto, N Nakamura, K Hirata, M Mizukoshi, T Akasaka, "Morphology of ExertionTriggered Plaque Rupture in Patients With Acute Coronary Syndrome - An Optical Coherence Tomography Study," Circulation, vol. 111, pp. 2368-2373, 2008.

[41] S Takarada, T Imanishi, T Kubo, T Tanimoto, H Kitabata, N Nakamura, A Tanaka, M Mizukoshi, T Akasaka, "Effect of statin therapy on coronary fibrous-cap thickness in patients with acute coronary syndrome: Assessment by optical coherence tomography study," Atherosclerosis, vol. 202, pp. 491-497, 2009.

[42] D Matsumoto, J Shite, T Shinke, H Otake, Y Tanino, D Ogasawara, T Sawada, OL Paredes, K Hirata, M Yokoyama, "Neointimal coverage of sirolimus-eluting stents at 6month follow-up: evaluated by optical coherence tomography," Eur. Heart J., vol. 28, pp. 961-967, 2007.

[43] GJ Czarnota, MC Kolios, J Abraham, M Portnoy, FP Ottensmeyer, JW Hunt, MD Sherar, "Ultrasound imaging of apoptosis: high-resolution non-invasive monitoring of 
programmed cell death in vitro, in situ and in vivo," Brit. J. Cancer, vol. 81, no. 3, pp. 520-527, 1999.

[44] JFR Kerr, AH Wyllie, AR Currie, “Apoptosis: A Basic Biological Phenomenon with Wide-ranging Implications in Tissue Kinetics,” Brit. J. Cancer, vol. 26, no. 4, pp. 239$257,1972$.

[45] JGH Joosten, JL McCarthy, "Dynamic and Static Light Scattering by Aqueous Polyacrylamide Gels," Macromolecules, vol. 24, pp. 6690-6699, 1991.

[46] FJ van der Meer, DJ Faber, MC Aalders, AA Poot, I Vermes, TG van Leeuwen, "Apoptosis- and necrosis-induced changes in light attenuation measured by optical coherence tomography," Laser Med. Sci., vol. 25, no. 2, pp. 259-267, 2010.

[47] SH Yun, C Boudoux, GJ Tearney, BE Bouma, "High-speed wavelength-swept semiconductor laser with a polygon-scanner-based wavelength filter," Opt. Lett., vol. 28, no. 20, pp.1981-1983, 2003.

[48] A Mariampillai, BA Standish, EH Moriyama, M Khurana, NR Munce, MKK Leung, J Jiang, A Cable, BC Wilson, IA Vitkin, VXD Yang, "Speckle variance detection of microvasculature using swept-source optical coherence tomography," Opt. Lett., vol. 33, no. 13, pp. 1530-1532, 2008. 
[49] A Mariampillai. Development of a High Resolution Microvascular Imaging Toolkit for Optical Coherence Tomography. Ph.D. Thesis. University of Toronto: Canada. 2010.

[50] RK Jain, "Transport of Molecules in the Tumor Interstitium: A Review," Cancer Res., vol. 47, pp. 3039-3051, 1987.

[51] Y Yamamoto, "Fundamentals of Noise processes," Stanford University, EEAP248 lecture notes, Chapter 1, 2011. 